\title{
Methods and Design Techniques for the Miniaturization of an L-Band System-on-Package Receiver Front-End
}

by

Grzegorz M. Brzezina, B.Eng, M.A.Sc.

\begin{abstract}
A dissertation submitted to the
Faculty of Graduate and Postdoctoral Affairs

in partial fulfillment of the requirements for the degree of
\end{abstract}

Doctor of Philosophy in Electrical and Computer Engineering

Ottawa-Carleton Institute for Electrical and Computer Engineering

Carleton University

Department of Electronics Engineering

July, 2011

(C)Copyright

Grzegorz M. Brzezina, 2011 


$\begin{array}{ll}\begin{array}{l}\text { Library and Archives } \\ \text { Canada }\end{array} & \begin{array}{l}\text { Bibliothèque et } \\ \text { Archives Canada }\end{array} \\ \begin{array}{l}\text { Published Heritage } \\ \text { Branch }\end{array} & \begin{array}{l}\text { Direction du } \\ \text { Patrimoine de l'édition }\end{array} \\ \begin{array}{l}\text { 395 Wellington Street } \\ \text { Ottawa ON K1A ON4 } \\ \text { Canada }\end{array} & \begin{array}{l}395, \text { rue Wellington } \\ \text { Ottawa ON K1A ON4 } \\ \text { Canada }\end{array}\end{array}$

Your file Votre référence

ISBN: 978-0-494-83241-7

Our file Notre référence

ISBN: 978-0-494-83241-7

NOTICE:

AVIS:

The author has granted a nonexclusive license allowing Library and Archives Canada to reproduce, publish, archive, preserve, conserve, communicate to the public by telecommunication or on the Internet, loan, distribute and sell theses worldwide, for commercial or noncommercial purposes, in microform, paper, electronic and/or any other formats.

The author retains copyright ownership and moral rights in this thesis. Neither the thesis nor substantial extracts from it may be printed or otherwise reproduced without the author's permission.

L'auteur a accordé une licence non exclusive permettant à la Bibliothèque et Archives Canada de reproduire, publier, archiver, sauvegarder, conserver, transmettre au public par télécommunication ou par l'Internet, prêter, distribuer et vendre des thèses partout dans le monde, à des fins commerciales ou autres, sur support microforme, papier, électronique et/ou autres formats.

L'auteur conserve la propriété du droit d'auteur et des droits moraux qui protège cette thèse. Ni la thèse ni des extraits substantiels de celle-ci ne doivent être imprimés ou autrement reproduits sans son autorisation.
In compliance with the Canadian Privacy Act some supporting forms may have been removed from this thesis.

While these forms may be included in the document page count, their removal does not represent any loss of content from the thesis.
Conformément à la loi canadienne sur la protection de la vie privée, quelques formulaires secondaires ont été enlevés de cette thèse.

Bien que ces formulaires aient inclus dans la pagination, il n'y aura aucun contenu manquant.

\section{Canadä}




\section{Abstract}

This thesis presents a highly miniaturized L-band receiver front end using a Systemon-Package (SoP) design approach that minimizes the trade-offs between size and performance. The receiver module contains embedded passive components as well as multiple silicon Integrated Circuits (ICs) to form a subsystem of a transceiver architecture. This allows for improved system performance and yields through the mixing of different device technologies and individually tested components. Size reduction is achieved through high-density three dimensional packaging. Inherent challenges of SoP designs include: i) parasitic coupling; ii) thermal management; iii) system interconnects; iv) manufacturing; and v) testing.

Highly miniaturized components are designed with a new methodology and realized in Low Temperature Co-fired Ceramic (LTCC) technology. A complete receiver front-end is designed through the combination of these components. Ansoft HFSS, an industry standard FEM-based electromagnetic solver, is employed in the design of the module and all embedded passive structures. The necessary active components (Low Noise Amplifier (LNA), mixer etc.) are implemented with commercial Surface Mount Technology (SMT) components.

Given the relatively low frequency for an L-band receiver (1525 $\mathrm{MHz}$ to $1575 \mathrm{MHz})$, a lumped-element approach is taken to implement the Bandpass Filter (BPF), 90 
degree hybrid coupler, and antenna balun. Measured results for the individual components and the complete receiver are shown to be in good agreement with the simulations.

This work is being undertaken in collaboration with SkyWave Inc. (formerly TransCore Inc.). 
I dedicate this work to the memory of my father, George Brzezina, who was an inspiration and is sorely missed. 


\section{Acknowledgments}

First and foremost, I wish to thank Professor L. Roy, my thesis supervisor in the Department of Electronics, for his invaluable guidance and support during my studies at Carleton University. He has always acted in a fair and professional manner. The always kind and supportive faculty and staff at Carleton University are too numerous to name but Professor L. MacEachern, in particular, provided me with important resources. I also wish to extend my gratitude to P. Lafleur, President of Resonance Microwave Systems Inc., for his important advice and honest opinions.

The completion of this work has been made possible by the assistance of many kind and talented individuals at the Communications Research Centre (CRC). I gratefully acknowledge the assistance of Dr. R. Amaya for giving me access to facilities that were essential to this thesis. Many thanks are extended to Ms. C. Glaser, Mr. S. Meilleur, Mr. A. Momciu, and Mr. J. Balbona, all from CRC, for donating their time and skills to aid my work. Similarly, I thank Mr. K. Oueng and Ms. J. Smithson from the Canadian Space Agency for being so helpful and generous with their time.

Special thanks is addressed to my colleagues from Carleton University, Dr. A. Shamim, Mr. B. Tamer, Dr. P. Chiurlyia, Mr. Z. El-Khatib, Dr. Z. Xu, Mr. L. Filipovic, and Mr. I. Miletic, with whom I have had enlightening conversations and many other enjoyable times. I also recognize the help I received form Ms. B. Neufeld 
in preparing the manuscript. Throughout the course of this work my mother, Ms. J. Brzezina, and my sister, Ms. B. Brzezina, have supported me without fail. To them I will always be sincerely grateful. Finally, I acknowledge my wife, Mrs. S. Brzezina, for her loving support and understanding.

The financial support provided by the National Science and Engineering Research Council of Canada and the Ontario Graduate Scholarship Program is gratefully acknowledged. 


\section{Table of Contents}

Abstract

Acknowledgments $\quad$ vi

Table of Contents viii

List of Tables $\quad$ xiii

List of Figures $\quad$ xvi

List of Acronyms $\quad$ xxiv

1 Introduction 1

1.1 Motivation and Challenges . . . . . . . . . . . . . 3

1.2 Research Objectives . . . . . . . . . . . . . . 5

1.3 Thesis Contributions . . . . . . . . . . . . . . 6

1.4 Thesis Organization . . . . . . . . . . . . . . 7

2 SoP Solution for System Integration $\quad 8$

2.1 System-Level Integration Background . . . . . . . . . . . . . . 8

2.2 Technology Medium . . . . . . . . . . . . . . . . 13

viii 


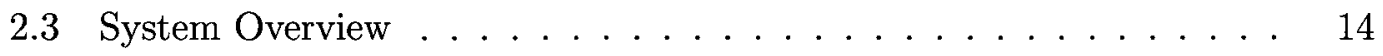

2.4 Bandpass Filter Background . . . . . . . . . . . 16

2.5 Microwave Coupler Background . . . . . . . . . . . . 18

2.6 Antenna Element Background . . . . . . . . . . . . . . . 20

2.7 Downconverter and Image Reject Mixer Background . . . . . . . . . . 24

2.8 Summary . . . . . . . . . . . . . . . . 26

3 BPF Design and Methodology $\quad 27$

3.1 Overview of Methodology . . . . . . . . . . . . . 28

3.2 LTCC Design Enhancement Methodology . . . . . . . . . . . . 28

3.3 Design Specifications and Circuit Schematic ........... 33

3.4 Passive Component Design . . . . . . . . . . . . . . . 35

3.4.1 Circular Spiral Inductor Design . . . . . . . . . . 35

3.4.2 Circular Multilayer Capacitor Design . . . . . . . . . . . 37

3.4.3 Novel L-C Resonator Design . . . . . . . . . . . . . . . . . 39

3.4.4 Coupling Capacitor Design . . . . . . . . . . . . . . 41

3.5 LTCC Bandpass Filter Design . . . . . . . . . . . . . . . 41

3.5.1 Hybrid Filter Structure - Four Ideal Coupling Capacitors . . . 41

3.5.2 Hybrid Filter Structure - Two Ideal Coupling Capacitors . . . 42

3.5.3 Fully Embedded Filter Structure . . . . . . . . . . 43

3.6 Passive Component and Bandpass Filter Characterization . . . . . . 44

3.6.1 Inductor Characterization ............... 44

3.6.2 Circular Multilayer Capacitor Characterization ....... 45

3.6.3 Novel L-C Resonator Characterization . . . . . . . . . . . 48

3.6.4 Coupling Capacitor Characterization . . . . . . . . . . . 49 
3.6.5 Filter Characterization . . . . . . . . . . . . . 50

3.7 LTCC Process Tolerance Effects . . . . . . . . . . . . . . . . 54

3.7.1 Influence of Layer Thickness Tolerance . . . . . . . . . . 54

3.7.2 Influence of Dielectric Constant . . . . . . . . . . 54

3.7.3 Influence of Metallization Quality . . . . . . . . 55

3.7.4 Post Simulated Results . . . . . . . . . . . . . . . . . 55

3.7 .5 Performance Summary . . . . . . . . . . . . . 55

3.8 Summary and Contribution $\ldots \ldots \ldots \ldots \ldots \ldots$

4 Advanced BPF Designs $\quad 58$

4.1 Finite Transmission Zeros . . . . . . . . . . . . . . . . . . . 59

4.1 .1 Capacitive Coupling $\ldots \ldots \ldots \ldots 60$

4.2 Challenges . . . . . . . . . . . . . . . . . . 62

4.3 Improved Second-Order Filter Design $\ldots \ldots \ldots \ldots \ldots$

4.3.1 Resonator Configuration . . . . . . . . . . . . . . 66

4.3.2 Improved Fourth-Order Filter Design . . . . . . . . . . 67

4.4 Component Library Creation and Simulated Filter Responses . . . . 69

4.4 .1 Second-Order Filter . . . . . . . . . . . . . . . . . . . . 69

$4.4 .2 \quad$ Fourth-Order Filter . . . . . . . . . . . . . . . . 72

4.5 Characterization of Improved Filters $\ldots \ldots \ldots \ldots \ldots$

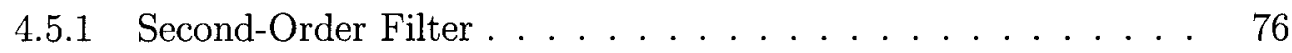

$4.5 .2 \quad$ Fourth-Order Filter . . . . . . . . . . . . . . . . . . 79

4.6 Comments on Simulation-Measurement Agreement . . . . . . . . 84

4.7 Summary and Contribution $\ldots \ldots \ldots \ldots \ldots$ 
5 90-Degree Hybrid Coupler Design $\quad 88$

5.1 Design Specifications and Circuit Schematic . . . . . . . . . . 89

5.2 Component Library Creation and Simulated Performance . . . . . . . 91

5.2 .1 Inductor Design . . . . . . . . . . . . . . . 92

5.2 .2 Capacitor Design .................... 94

5.3 Coupler Simulated Performance . . . . . . . . . . . . . . 97

5.4 Hybrid Coupler Characterization . . . . . . . . . . . . . 101

5.5 Summary and Contribution . . . . . . . . . . . 107

6 180-Degree Hybrid Coupler (Balun) Design 109

6.1 Design Specifications and Circuit Schematic . . . . . . . . . . . 111

6.2 Component Library Creation and Simulated Performance . . . . . . . 113

6.2 .1 Inductor Design . . . . . . . . . . . . . 113

6.2 .2 Capacitor Design . . . . . . . . . . . . . 115

6.3 Balun Simulated Performance . . . . . . . . . . . . . . 116

6.4 Balun Characterization . . . . . . . . . . . . . . . . 119

6.5 Summary and Contribution . . . . . . . . . . . . 127

7 Antenna Element Design 130

7.1 Antenna Concept and Initial Design . . . . . . . . . . . . . . . 131

7.2 Antenna Design and Optimization . . . . . . . . . . . . 133

7.3 Matching Network . . . . . . . . . . . . . . . . . 136

7.4 Simulated Results of Final Design . . . . . . . . . . . . . . . . 138

7.4 .1 Ideal Feed . . . . . . . . . . . . . . . . . 138

7.4 .2 Balun Feed . . . . . . . . . . . . . 140

7.5 Measured Results . . . . . . . . . . . . . . . . . . 143 
7.6 Summary and Contribution . . . . . . . . . . . 153

8 SoP Design of an L-Band Miniature Receiver Module for GPS 157

8.1 GPS Specifications . . . . . . . . . . . . . . . 158

8.2 Receiver Requirements . . . . . . . . . . . . . . 159

8.2 .1 Gain ......................... 159

8.2 .2 Noise Figure . . . . . . . . . . . . . . . . . . 159

8.2.3 Image Rejection Ratio . . . . . . . . . . . . . . . 161

8.3 MRM System Architecture . . . . . . . . . . . . . . . . 162

8.3.1 Basic Functionality . . . . . . . . . . . . 163

8.3.2 Detailed Circuit Schematic . . . . . . . . . . . . . 164

8.4 MRM Layout Design . . . . . . . . . . . . . . . . . . 167

8.5 Active Component Selection . . . . . . . . . . . . . 170

8.6 Fabricated MRM . . . . . . . . . . . . . 175

8.7 MRM Testing . . . . . . . . . . . . . . . . . 175

8.8 Summary and Contribution . . . . . . . . . . . . 181

9 Thesis Summary and Suggestions for Future Work 183

$\begin{array}{lr}\text { List } \text { of References } & 189\end{array}$ 


\section{List of Tables}

2.1 System Integration Methods Overview . . . . . . . . . . . . 12

2.2 A Comparison of Material Systems . . . . . . . . . . . . . 13

2.3 LTCC Material Properties . . . . . . . . . . . . . . 15

2.4 Antenna Element Survey . . . . . . . . . . . . . . . 23

3.1 Theoretical Component Values For Bandpass Filters . . . . . . . . . . 34

3.2 LTCC Material Properties . . . . . . . . . . . . . . . 35

3.3 Comparison Between Initial and Optimized Component Dimensions . 43

3.4 Performance Summary . . . . . . . . . . . . . . . . 56

4.1 Filter Specifications . . . . . . . . . . . . . . . . 63

4.2 Theoretical Component Values For Improved Second-Order Bandpass Filter ......................... 64

4.3 Theoretical Component Values For Improved Fourth-Order Bandpass Filter ......................... 67

4.4 Improved Second-Order Filter Simulated Performance . . . . . . . . 72

4.5 Improved Fourth-Order Filter Simulated Performance . . . . . . . . . 75

4.6 Measurement Summary - Second-Order Filter Components . . . . . . 79

4.7 Results Comparison - Improved Second-Order Filter . . . . . . . . . . 80

4.8 Measurement Summary - Fourth-Order Filter Components . . . . . . 82 
4.9 Results Comparison - Improved Fourth-Order Filter . . . . . . . . . . 84

4.10 Comparison With Related Work . . . . . . . . . . . . . 85

4.10 Comparison With Related Work . . . . . . . . . . . . 86

4.10 Comparison With Related Work . . . . . . . . . . . . . . . . 87

5.1 Component Optimization Results for Lumped Hybrid Coupler . . . . 99

5.2 Measurement Summary - Hybrid Coupler Components . . . . . . . . 105

5.3 Hybrid Coupler Measurement Summary . . . . . . . . . . . . 106

5.4 Comparison with Commercial Components . . . . . . . . . . . 108

6.1 Balun Optimization Results . . . . . . . . . . . . . . 118

6.2 Balun Measurement Configurations . . . . . . . . . . . . . . 121

6.3 Balun Measurement Summary . . . . . . . . . . . . . . . . . . 122

6.4 Measurement Summary - Balun Components . . . . . . . . . . . . . . 124

6.5 Comparison with Commercial Components . . . . . . . . . . 128

7.1 Final values for antenna design variables . . . . . . . . . . . 136

7.2 Differences in dipole performance when using an ideal port or embedded balun feed . . . . . . . . . . . . . . . . . . . . 144

7.3 A comparison of radiation efficiencies and peak gains . . . . . . 153

7.4 Comparison with Commercial GPS Antennas . . . . . . . . . . 155

8.1 GPS Specifications . . . . . . . . . . . . . . . 158

8.2 Active Antenna Specifications . . . . . . . . . . . . 160

8.3 Summary of Receiver Requirements . . . . . . . . . . . . . . . 162

8.4 MRM Layer Descriptions . . . . . . . . . . . . . . . . . . 169

8.5 MRM Bill of Materials . . . . . . . . . . . . . . . . . 172

8.5 MRM Bill of Materials . . . . . . . . . . . . . . . 173

8.5 MRM Bill of Materials . . . . . . . . . . . . . . . . . . 174

xiv 
8.6 CM Performance Results (Typical) . . . . . . . . . . . . . 177

8.7 MRM Performance Results (Typical) . . . . . . . . . . . . . . . 179 


\section{List of Figures}

1.1 Spectrum window for mobile broadband and corresponding component

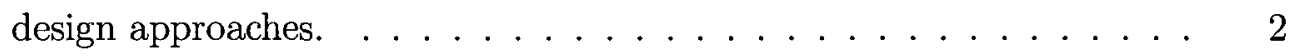

2.1 The SoC approach to system integration locates analog electronics, digital electronics, filters, and other passive components on chip. . . . 9

2.2 The SiP approach to system integration combines multiple specialized ICs in one package. . . . . . . . . . . . . . . . . 10

2.3 The SoP approach to system integration combines multiple specialized ICs with an advanced package that also provides many system functions. 11

2.4 Proposed system architecture. . . . . . . . . . . . . . . . . . . 14

2.5 Lumped element schematic of bandpass filter. . . . . . . . . . 17

2.6 Symmetric lumped element equivalent models: (a) 90-degree hybrid coupler and; (b) 180-degree coupler. . . . . . . . . . . . . . . 19

2.7 A survey of the antenna elements considered here for a GPS SoP. . . 21

3.1 Bandpass filter as part of the complete receiver chain. . . . . . . . . 27

3.2 Bandpass filter: (a) three dimensional structure and; (b) its equivalent schematic. . . . . . . . . . . . . . . . . . 29

3.3 Lumped element bandpass filter structure with four ideal capacitors replaced in the final implementation. . . . . . . . . . . . 31 
3.4 Flow chart comparison of (a) the proposed design methodology, and (b) the conventional approach. . . . . . . . . . . . . . 32

3.5 Lumped element bandpass filter structure with two ideal coupling ca-

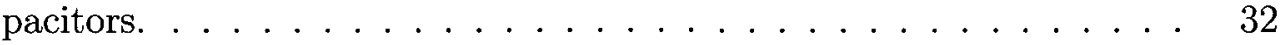

3.6 Lumped element schematic of bandpass filter. . . . . . . . . . 33

3.7 The relationship between inductance (L) and Q-factor for a spiral in-

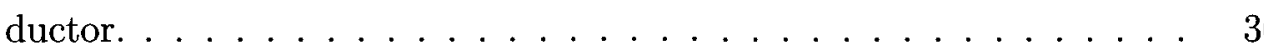

3.8 Spiral inductor model view from: (a) top and; (b) oblique. As indicated, the diameter is $1.8 \mathrm{~mm} \ldots \ldots \ldots$. . . . . . . . . 37

3.9 Gains in capacitance with the use of multiple layers. . . . . . . . . . . 38

3.10 Multilayer capacitor: (a) model with identical radii for each plate and; (b) simplified equivalent circuit.

3.11 L-C resonator model view from (a) top and (b) oblique. As indicated, the diameter is $1.8 \mathrm{~mm} \ldots \ldots \ldots$. . . . . . . . . . . . 40

3.12 The effect on resonator $\mathrm{Q}$ when increasing the separation between the parallel inductor and capacitor. . . . . . . . . . . . . 4 40

3.13 Measured and simulated Q-factor for inductor. . . . . . . . . . . . . . 44

3.14 Inductor metallization micrograph. . . . . . . . . . . . . . . 45

3.15 Measured and simulated inductance. . . . . . . . . . . . . . 46

3.16 Measured and simulated capacitance of C3. . . . . . . . . . . . 47

3.17 Measured and simulated Q-factor of C3. . . . . . . . . . . . . 47

3.18 Measured and simulated resonator Zin. . . . . . . . . . . . . . 48

3.19 Measured and simulated coupling capacitors of fully embedded filter. $\quad 49$

3.20 Measured and simulated response for filter with 4 surface mount capacitors. ........................ 50 
3.21 Micrograph of affixed surface mount coupling capacitors. . . . . . . 51

3.22 Simulated insertion loss variation due to $\mathrm{C} 1$ tolerance. . . . . . . . . 52

3.23 Micrograph of fabricated fully embedded bandpass filter (top view). . 52

3.24 Measured and simulated response for fully embedded filter. . . . . . . 53

3.25 A comparison of the measured, simulated, and post-simulated results for fully embedded filter. . . . . . . . . . . . . . 56

4.1 Bandpass filter as part of the complete receiver chain. . . . . . . . . 58

4.2 Second-order filter with no feedback (schematic and typical response). $\quad 60$

4.3 Second-order filter with capacitive feedback showing two finite transmission zeros (schematic and typical response). . . . . . . . . . 61

4.4 Fourth-order filter with inductive coupling (solid line) and without (dotted line) (schematic and typical response). . . . . . . . . . . 62

4.5 Improved Second-order filter with inductive and capacitive coupling. .

4.6 Simulated inductor coupling: (a) model; and (b) plot of coupling strength for various separation distances. . . . . . . . . . . . .

4.7 Three dimensional layout of new second-order filter: (a) oblique view; and $(\mathrm{b})$ top view. . . . . . . . . . . . . . . . 66

4.8 Fourth-order filter with inductive coupling. . . . . . . . . . . 67

4.9 Three dimensional layout of new fourth-order filter: (a) oblique view; and $($ b) top view. . . . . . . . . . . . . . . . . . 68

4.10 Resonator inductor simulated results for improved second-order filter: (a) inductance; and (b) quality factor. . . . . . . . . . . . . 70

4.11 Resonator capacitor simulated results for improved second-order filter: (a) capacitance; and (b) quality factor. . . . . . . . . . . . . 70 
4.12 Simulated improved second-order filter response: (a) wideband view; and (b) narrowband view. . . . . . . . . . . . 71

4.13 Resonator inductor simulated results for improved fourth-order filter: (a) inductance; and (b) quality factor. . . . . . . . . . . 73

4.14 Simulated capacitance and Q-factor for: (a)-(b) C4; and (c)-(d) C5. . 74

4.15 Simulated improved fourth-order filter response: (a) wideband view; and (b) narrowband view. . . . . . . . . . . . . 75

4.16 Comparison of measured and simulated second-order filter response: (a) wideband view; and (b) narrowband view. . . . . . . . . 77

4.17 Resonator inductor results comparison: (a) inductance; and (b) Q-factor. 77

4.18 Resonator capacitor results comparison: (a) capacitance; and (b) Q-

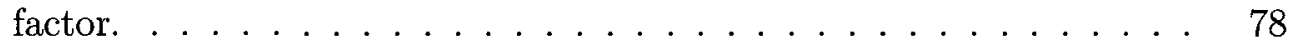

4.19 Comparison of measured and simulated fourth-order filter response: (a) wideband view; and (b) narrowband view. . . . . . . . . . 81

4.20 Fourth-order filter resonator results: (a) inductance; and (b) Q-factor. 81 4.21 Comparison of capacitance and Q-factor for: (a)-(b) C4; and (c)-(d) C5. 83

5.1 Hybrid coupler as part of the complete receiver chain. . . . . . . . . 88

5.2 Classical distributed coupler layout. . . . . . . . . . . . 89

5.3 Symmetric lumped element schematic for hybrid coupler. . . . . . . . 90

5.4 Multilayer spiral inductor structures: (a) L1; and (b) L2. . . . . . . . 92

5.5 Multilayer spiral inductor structure: (a) length tuning; and (b) its effect on SRF and inductance at $1.55 \mathrm{GHz}$ (all other dimensions un-

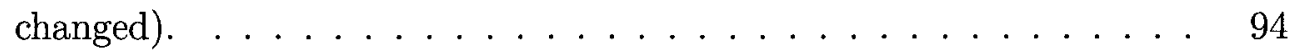

5.6 Simulated inductance and Q-factor for: (a)-(b) L1; and (c)-(d) L2. . 95 
5.7 Asymmetric multilayer capacitor structure: (a) oblique view; and (b) dimensions. . . . . . . . . . . . . . . . 96

5.8 Asymmetric multilayer capacitor simulated performance: (a) capacitance; and (b) Q-factor. . . . . . . . . . . . . 97

5.9 Hybrid coupler structure: (a) three dimensional view; and (b) top view. 98

5.10 Simulated coupler performance: (a) $S_{11}$; (b) $S_{21}$; (c) $S_{31}$; and (d) combined $\Delta \theta$ and amplitude imbalance. . . . . . . . . . . . . 100

5.11 Fabricated coupler: (a) original component; and (b) modified test setup.101

5.12 A comparison of measured and simulated coupler performance: (a) $S_{11}$;

(b) $S_{21} ;$ (c) $S_{31}$; and (d) combined $\Delta \theta$ and amplitude imbalance. . . 102

5.13 Asymmetric multilayer capacitor measured performance: (a) capacitance; and (b) Q-factor. . . . . . . . . . . . . . 103

5.14 Measured inductance and Q-factor for: (a)-(b) L1; and (c)-(d) L2. . . 104

6.1 Bandpass filter as part of the complete receiver chain. . . . . . . . . 109

6.2 Classical distributed rat-race coupler layout. . . . . . . . . . . . . . . 112

6.3 Symmetric lumped element equivalent model for rat-race coupler. . . 112

6.4 Multilayer spiral inductor structures: (a) three-dimensional model; and (b) length adjustment.

6.5 Simulated inductor performance for balun: (a) inductances and; (b)

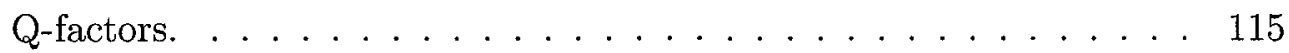

6.6 Simulated capacitor performance for balun: (a) capacitances; and (b)

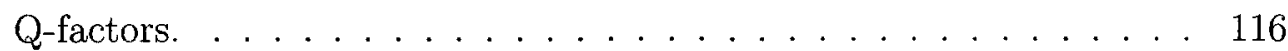

6.7 Balun structure: (a) three dimensional view; and (b) top view. . . . . 117

6.8 Magnetic fields within balun structure. . . . . . . . . . . . . . . . 119 
6.9 Simulated balun performance: (a) $S_{11}$; (b) $S_{22}$ and $S_{21}$; (c) $S_{33}$ and $S_{31} ;$ (d) $S_{44}$ and $S_{41} ;$ and (e) combined $\Delta \theta$ and amplitude imbalance. 120

6.10 Micrograph of fabricated LTCC balun. . . . . . . . . . . . . . . . . 121

6.11 Measured balun performance: (a) $S_{11}$; (b) $S_{22}$ and $S_{21}$; (c) $S_{33}$ and $S_{31}$; (d) $S_{44}$ and $S_{41}$; and (e) combined $\Delta \theta$ and amplitude imbalance. . . . 123

6.12 Measured inductance and Q-factor for: (a)-(b) $L 1$; (c)-(d) L2; and (e)-(f) $L 3 . \ldots \ldots$. . . . . . . . . . . . . . . . . . . . . . . . . 125

6.13 Measured 2C1 performance: (a) inductance and; (b) Q-factor. . . . . 126

6.14 A comparison of the measured, simulated, and post-simulated results for the balun. . . . . . . . . . . . . . . . 126

7.1 Antenna as part of the complete receiver chain. . . . . . . . . . 130

7.2 Miniaturization of a conventional dipole by the meandering of each arm: (a) concept; and (b) realization. . . . . . . . . . . . 133

7.3 Comparison of input resistances for two different length dipoles. . . . 134

7.4 Proposed miniaturized dipole layout. . . . . . . . . . . . 135

7.5 L-section matching network for: (a) single-ended; and (b) differential antennas.

7.6 A comparison of the return loss for the SoP dipole with and without a matching network.

7.7 (a) Coordinate system used for radiation patterns; (b) Simulated 3dimensional result referenced to antenna model. . . . . . . . . . . . . 140

7.8 Simulated radiation patterns with ideal port excitation and lossless matching elements. Co-pol (solid line) and cross-pol (dashed line) results are shown. . . . . . . . . . . . . . . . 141

7.9 Location of (a) lumped element balun; and (b) detailed image of balun. 142 
7.10 Simulated radiation patterns with balun feed. Co-pol (solid line) and cross-pol (dashed line) results. . . . . . . . . . . . . . . . . . . 143

7.11 Performance difference for ideal and balun fed dipole. . . . . . . . . . 144

7.12 Micrograph of fabricated SoP antenna: (a) top; and (b) bottom view. 146

7.13 GPS module configured for dipole return loss measurement. . . . . . 147

7.14 Measured antenna return loss for various values of matching capacitors. 147

7.15 Photographs of anechoic chamber used to obtain radiation patterns. Image showing (a) both antennas; and (b) close-up of antenna under

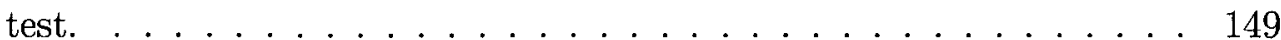

7.16 Experimental azimuth (Z-Y plane) radiation patterns. Co-pol (solid line) and cross-pol (dashed line) results. . . . . . . . . . . . 150

7.17 Experimental elevation (Y-X plane) radiation patterns. Co-pol (solid line) and cross-pol (dashed line) results. . . . . . . . . . . . . . 151

7.18 Comparison of principal plane radiation patterns at $1.507 \mathrm{GHz}$. Measured (solid line) and simulated (dashed line) results: (a) azimuth; and (b). Elevation . . . . . . . . . . . . . . . . . 154

8.1 Active components of the miniature receiver module (MRM) . . . . . 157

8.2 Block diagram of the active antenna which constitutes the first element in the receiver. . . . . . . . . . . . . 160

8.3 Image rejection ratio for different values of phase and amplitude imbalance from Equation 8.3. . . . . . . . . . . . . . . 161

8.4 A quadrature downconverter with a Hartley image reject mixer and RF path quadrature generation. . . . . . . . . . . . . . 164

8.5 Detailed schematic of MRM without antenna and balun stages. . . . 165

8.6 Low drop-out power regulator for MRM. . . . . . . . . . . . . . 167 
8.7 Area allocation for the various components of the MRM. . . . . . . 168

8.8 Locations of MRM components. . . . . . . . . . . . . . . . . . . 169

8.9 Selected metal layers of the MRM. . . . . . . . . . . . . . . 171

8.10 Micrograph of fabricated MRM with populated components: (a) top; and (b) bottom view. . . . . . . . . . . . . . . 175

8.11 MRM and Skywave Inc. measurement setup. . . . . . . . . . . . 176

8.12 Spectrums of MRM IF outputs (low side LO): (a) I; and (b) Q. . . . 178

8.13 MRM filter measurements: (a) normalized $S_{21}$; and (b) comparison

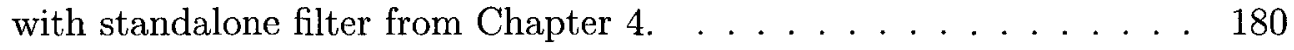

8.14 MRM 90-degree coupler measurements: (a) phase difference; and (b) amplitude imbalance. . . . . . . . . . . . . . . . . 181 


\section{List of Acronyms}

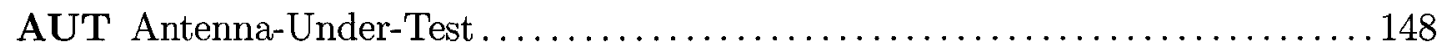

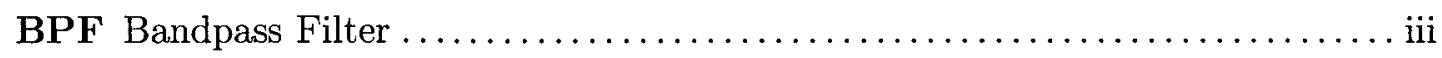

BPSK Binary Phase-Shift Keying $\ldots \ldots \ldots \ldots \ldots \ldots \ldots \ldots \ldots \ldots \ldots \ldots \ldots \ldots \ldots \ldots \ldots \ldots \ldots \ldots \ldots$

CMOS Complementary Metal Oxide Semiconductor $\ldots \ldots \ldots \ldots \ldots \ldots \ldots \ldots$

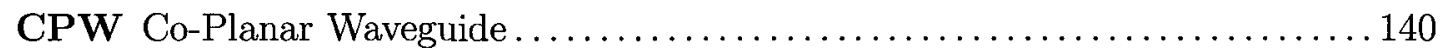

DSSS Direct-Sequence Spread Spectrum........................ 158

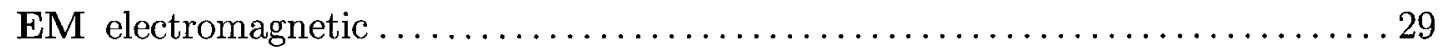

FCC Federal Communication Commission $\ldots \ldots \ldots \ldots \ldots \ldots \ldots \ldots \ldots \ldots \ldots \ldots \ldots \ldots \ldots$

GPS Global Positioning System $\ldots \ldots \ldots \ldots \ldots \ldots \ldots \ldots \ldots \ldots \ldots \ldots \ldots \ldots \ldots \ldots \ldots \ldots \ldots \ldots \ldots$

GSM Global System for Mobile Communications $\ldots \ldots \ldots \ldots \ldots \ldots \ldots \ldots \ldots$

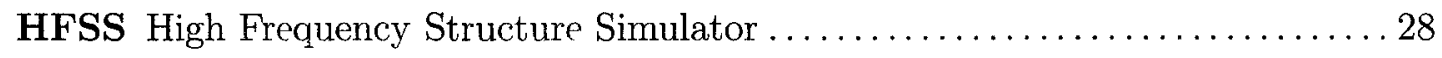

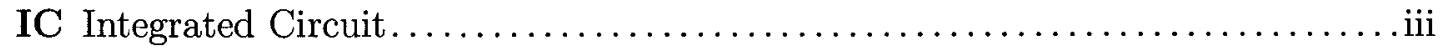

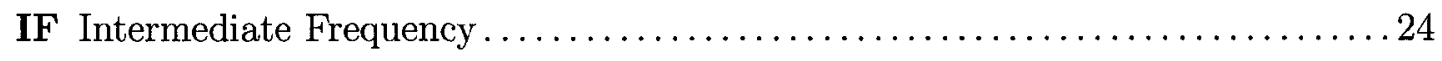

xxiv 
IMT International Mobile Telecommunications......................

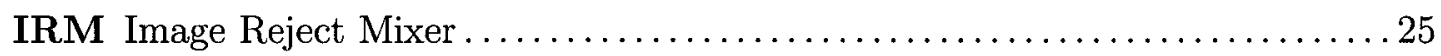

IRR Image Rejection Ratio............................... 161

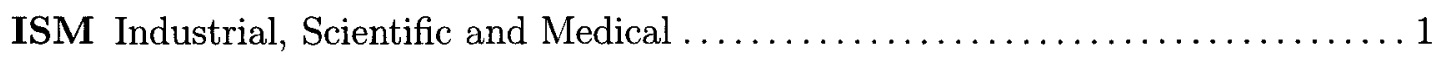

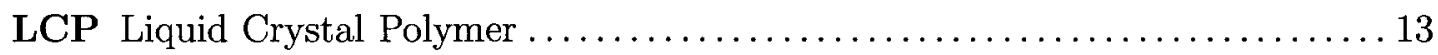

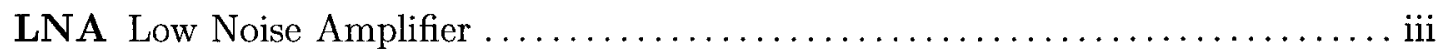

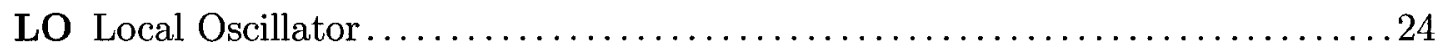

LTCC Low Temperature Co-fired Ceramic ........................

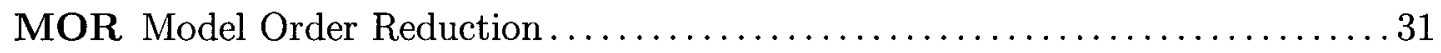

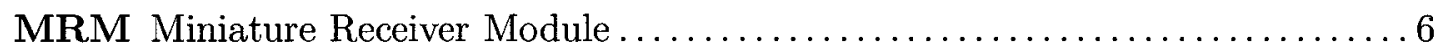

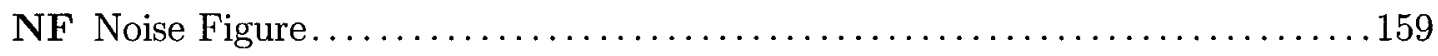

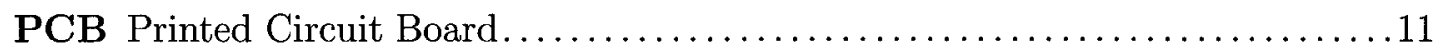

PCS Personal Communications Service $\ldots \ldots \ldots \ldots \ldots \ldots \ldots \ldots \ldots \ldots \ldots \ldots \ldots \ldots \ldots$

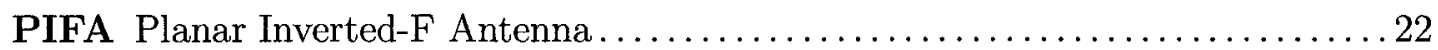

QVCO Quadrature Voltage Controlled Oscillator ..................... 24

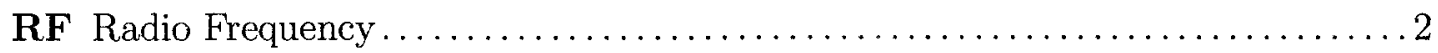

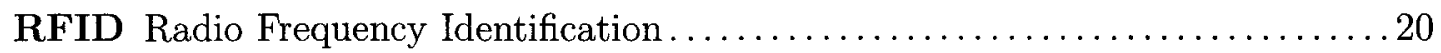

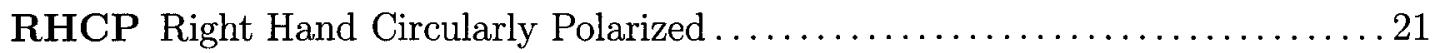


SD Secure Digital......................................

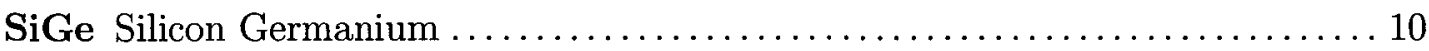

Si-MMIC Silicon - Monolithic Microwave Integrated Circuit.............. 172

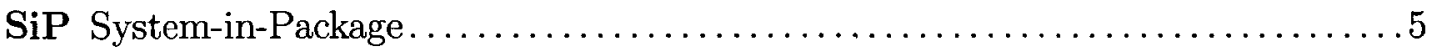

SMT Surface Mount Technology $\ldots \ldots \ldots \ldots \ldots \ldots \ldots \ldots \ldots \ldots \ldots \ldots \ldots \ldots \ldots \ldots \ldots \ldots$

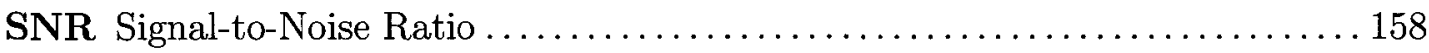

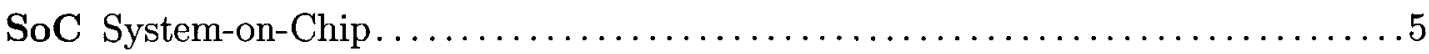

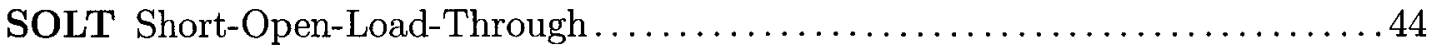

SoP System-on-Package $\ldots \ldots \ldots \ldots \ldots \ldots \ldots \ldots \ldots \ldots \ldots \ldots \ldots \ldots \ldots \ldots \ldots \ldots \ldots \ldots \ldots \ldots$

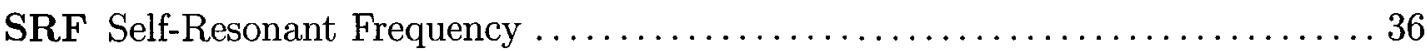

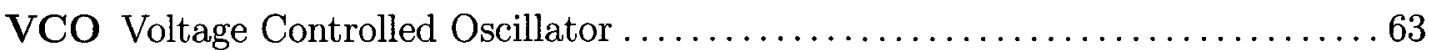

VNA Vector Network Analyzer ................................ 44

WLAN Wireless Local Area Network.............................. 22 


\section{Chapter 1}

\section{Introduction}

Increasingly, social and economic improvements are associated with the availability of broadband mobile communications. Worldwide demand is growing and consequently today's limited spectrum is becoming very crowded. Some have called this situation a "spectrum famine" that threatens to slow or even stop further advancements in mobile communications [1]. The ultimate reason is that the optimum frequency range for mobile communications is relatively narrow, stretching from about $300 \mathrm{MHz}$ to $3.5 \mathrm{GHz}$.

This window is especially crowded above $1700 \mathrm{MHz}$, where the Global System for Mobile Communications (GSM), Personal Communications Service (PCS), International Mobile Telecommunications (IMT), and Industrial, Scientific and Medical (ISM) bands are located. Therefore, regulators are looking lower in frequency and at legacy applications that can be deleted or re-allocated elsewhere. This has been demonstrated recently with the phasing out of analog TV $(470-862 \mathrm{MHz})$ thereby creating a "digital dividend" in which $108 \mathrm{MHz}$ of unallocated bandwidth was freed up by replacing analog TV with digital TV broadcasts. The available spectrum can be used to fuel new mobile sector growth. Indeed, in the United States the Federal 
Communication Commission (FCC) has auctioned part of this new spectrum around $700 \mathrm{MHz}$ for future $4 \mathrm{G}$ communications.

In radio transceiver design, the frequency of operation dictates the method of realization for passive components, whose quality factors are critical to product success. At higher frequencies distributed elements dominate. However, as we move lower in frequency, the wavelength becomes correspondingly longer and distributed elements become too large to be practical. This situation is illustrated in Fig. 1.1. Below $1.5 \mathrm{GHz}$ is the domain where lumped elements are primarily needed to make Radio Frequency (RF) and microwave circuits small, and cost effective [2]. However, RF lumped elements have notoriously poor quality factors, limiting circuit efficiency [3]. The achievement of efficient L-band ( $1 \mathrm{GHz}$ to $2 \mathrm{GHz}$ ) componentry would therefore serve as a gateway to lower frequency applications that utilize lumped elements.

Mobile broadband ( $300 \mathrm{MHz}$ to $3.5 \mathrm{GHz})$

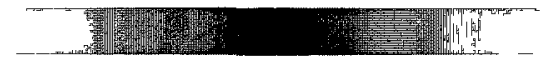

Distributed elements

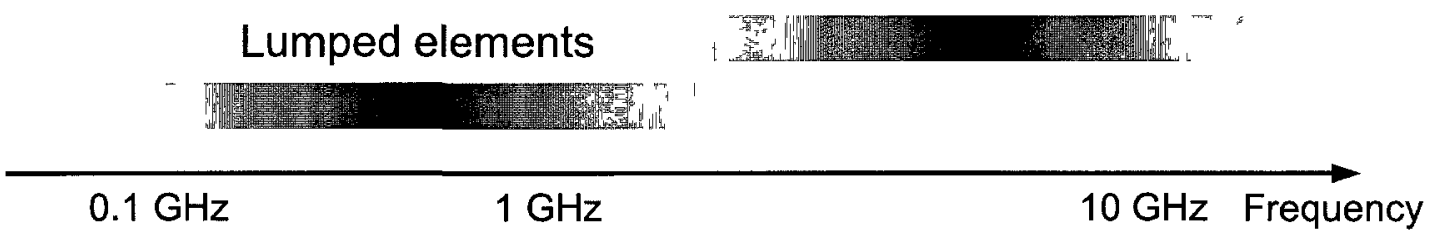

Figure 1.1: Spectrum window for mobile broadband and corresponding component design approaches.

L-band microwave circuit miniaturization is an important requirement for emerging portable wireless applications. In particular, Global Positioning System (GPS) $(1.575 \mathrm{GHz})$ is quickly expanding from its military origins into the general user market, not only as a stand-alone product but also as a valuable feature in cellular phones, 
cameras, netbooks, and other personal electronics. In Europe and Japan, GPS product sales have made a breakthrough and GPS devices are becoming commonplace in vehicles and handsets. By some estimates, the number of GPS-enabled smart phones is set to triple to 560 million handsets worldwide by 2012 [4]. In the past GPS devices were large and bulky. Today, a GPS device must be highly miniaturized to be conpatible with modern compact electronics. In this chapter, the motivation for this work along with the related objectives and challenges will be described. Also, the contributions of this work to the field of Electrical Engineering will be listed. Finally, thesis organization will be described.

\subsection{Motivation and Challenges}

The three motivating factors for the realization of an improved GPS receiver module (in order of importance) will be i) efficiency, ii) miniaturization, and iii) commercialisation potential. Each component of the receiver described in this thesis will be unique and include improvements to the current state-of-the-art that will classify this work as novel. Pursuing a design that has the highest degree of miniaturization without compromising efficiency will increase the relevance of this work in both academic and commercial contexts. Since this work is being undertaken with the cooperation of an industrial partner (SkyWave Inc.), commercialization is an important motivating factor. It will be a key goal to include as much practicality into the design as possible. The main challenges associated with achieving these goals are listed below:

1. Maintaining system-on-package (SoP) format compatibility.

The SoP format is very versatile. Nonetheless, methods and techniques must be used that result in component designs that efficiently leverage its advantages. 
For example, an edge-coupled filter would be a poor topology choice since only a single layer of a multi-layer substrate would be utilized, the remainder being wasted.

2. Overcoming the size performance trade-off.

This is a common reality in the field of microwave engineering. Clever methods and intelligent optimization techniques are required to minimize the penalties of shrinking passive components.

3. The design of high $\mathrm{Q}$ passive components.

Filters, baluns, and couplers are just some of the passive components that rely on low-loss capacitors and inductors. The latter component is typically the most difficult to design with high Q. Careful topology selection is needed to maximize Q.

4. Antenna packaging that is both compact and efficient.

Antennas are most efficient when their size is comparable to a wavelength. As such, they can easily be the largest component of a transceiver. Integration onto an IC results in low efficiencies, while opting for an external model can be prohibitively large and expensive. Therefore, a technique to embed the antenna within the SoP substrate is highly desirable.

5. Shielding and minimization of parasitic and mutual coupling.

Unwanted mutual coupling and other parasitic effects are a consequence of highly integrated designs. They can be highly detrimental to the overall performance of the system. Good shielding practices and careful layout techniques can minimize these effects. 
6. Package and interconnect design.

To link all system components, low loss interconnects are required. These connections should also be isolated from external radiation sources and create a minimum of spurious radiation.

\section{$1.2 \quad$ Research Objectives}

This work is undertaken with the cooperation of SkyWave Inc., a leading provider of satellite communications products for asset tracking. The objective of this work is to advance the state of the art of L-band receiver modules through a SoP implementation of a GPS receiver front-end whose dimensions are significantly smaller than current designs. Central to accomplishing this objective is the task of combining different technologies to optimize performance, reduce cost, and maximize the potential for commercialization. To this end, the need for practical SoP design guidelines will be addressed. LTCC multilayer technology is utilized in the design of all passive components and the containing module. The justification for this decision is provided in the next chapter. The specific research objectives of this work are as follows:

1. Investigate and compare SoP versus system-in-package System-in-Package (SiP) and System-on-Chip (SoC) design philosophies as applied to the design of Lband receiver components.

2. Formulation and application of a design methodology for a miniaturized component bandpass filter.

3. Design of novel embedded passive components (filters, couplers, baluns, etc.) based on the above methodology. 
4. Co-design and aggressive miniaturization of a SoP receiver front-end with antenna.

\subsection{Thesis Contributions}

The major contributions of this resulting from the successful fulfilment of the research objectives are listed below:

1. Novel Embedded L-C resonator.

Highest demonstrated Q-factor for an L-band LTCC inductor. This, combined in a novel way with a parallel capacitor, forms a new L-C resonator topology.

2. A novel three-dimensional lumped-element circuit design methodology.

A novel methodology is given and demonstrated with a filter design example. This new procedure reduces the time and resources required to create a new component.

3. A suite of new miniature LTCC passive components.

The smallest reported L-band filter, 90-degree hybrid coupler and balun components are demonstrated. Extreme miniaturization is achieved through novel packaging using standard low permittivity LTCC substrate material. This allows them to be embedded directly within the SoP module.

4. SoP integration of GPS receiver front-end with antenna to create a Miniature Receiver Module (MRM)).

The smallest implementation of a GPS receiver front-end that includes an integrated antenna is reported. A new balun-dipole combination is designed to 
be embedded within the substrate. The module as a whole is suitable for mass production and can be easily augmented with tunability features to compensate for components and fabrication tolerances.

To date, one journal article [5] and four conference papers have been published that are directly related to this work [6-9].

\subsection{Thesis Organization}

This thesis is organized in a way that follows the design flow for the GPS SoP. In Chapter 1 an introduction of the thesis topic and a description of the research objectives and contributions are provided. The concept of a SoP GPS receiver front-end is presented in Chapter 2 along with a detailed literature review of all system components. The concept shows how all the system components will be arranged and packaged into one compact module. Chapter 3 discusses the design methodology and implementation of the bandpass filter. In this chapter a novel lumped element L-C resonator structure is presented for the first time. In Chapter 4, two new bandpass filters are designed and characterized which improve upon the previous results. The next passive component in the receiver chain is the 90-degree hybrid coupler whose design and measurement is discussed in Chapter 5. A similar structure to the quadrature hybrid is the balun that is described in Chapter 6. The balun is used to convert the differential antenna output to a single-ended signal. Details of the dipole antenna design and characterization are given in Chapter 7 . With the major passive components described, Chapter 8 focuses on the implementation of the complete SoP module. Finally, in Chapter 9 a summary of the thesis is provided along with a plan for future work. 


\section{Chapter 2}

\section{SoP Solution for System Integration}

In accordance with objective 1, a comparison between $\mathrm{SoP}, \mathrm{SoC}$ and $\mathrm{SiP}$ is undertaken. These three popular approaches to system-level integration are discussed along with an overview of three important system blocks: i) bandpass filter, ii) hybrid coupler, and $i i i)$ antenna. This chapter also presents an overview of the proposed miniature receiver front-end along with a literature review. A GPS application is chosen partly because its design can benefit greatly from system-level integration techniques. The overall system architecture will be shown here and each subsystem will be described in detail later.

\subsection{System-Level Integration Background}

As mentioned above, three different methods exist for achieving system-level integration: SoC, SiP, and SoP. All of these methods have cost and miniaturization as primary goals, while SoP adds performance improvement to the list. More specifically, the SoC method integrates all functionality onto one integrated circuit. As shown in Fig. 2.1, this includes analog and digital circuitry along with passive components. 


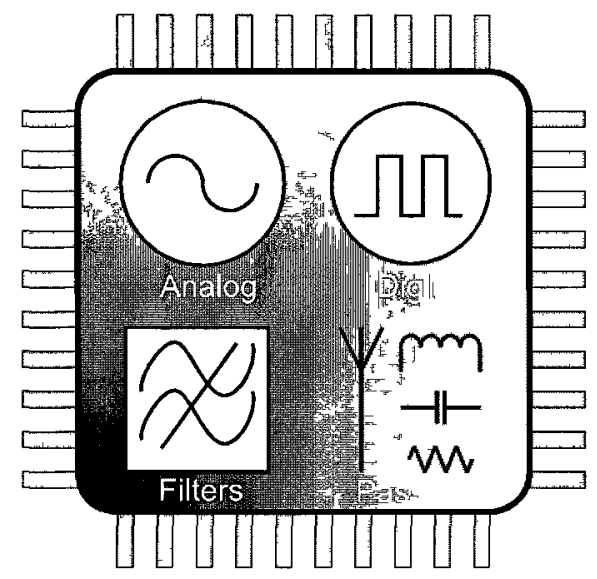

Figure 2.1: The SoC approach to system integration locates analog electronics, digital electronics, filters, and other passive components on chip.

Managing to contain all of these system components in a single IC minimizes cost by leveraging the benefits of mass production. To realıze this benefit, however, an advanced manufacturing process is required. Moreover, mixing so many different types of electronics into a single integrated circuit usually necessitates performance compromises. One such compromise, inherent to all low resistivity bulk Complementary Metal Oxide Semiconductor (CMOS) processes, is the limitation of inductor Q-factors to below 20 precludes SoC as an option for this work [10]. The Other SoC challenges

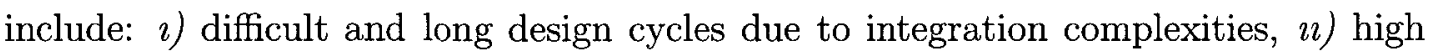
fabrication costs due to different process requirement of analog and digital circuits, and $\imath 2$ ) intellectual property issues [11]. Until these formidable challenges are overcome, SoC technology will remain a unfavorable solution to the system integration problem

The SiP method addresses some of the drawbacks of SoC technology by making it possible to combine separate ICs on one package substrate [12]. This allows for the partitioning of the system into separate blocks that can each use uniquely optimized 
fabrication technologies. This ability removes a difficult decision that a $\mathrm{SoC}$ designer would have to make between compatibility and performance. For example, high speed digital processors can be fabricated in leading edge CMOS technology, while sensitive $\mathrm{RF}$ analog electronics may be realized in Silicon Germanium (SiGe) technology. Most SiP modules vertically stack ICs to minimize overall size as displayed in Fig. 2.2.

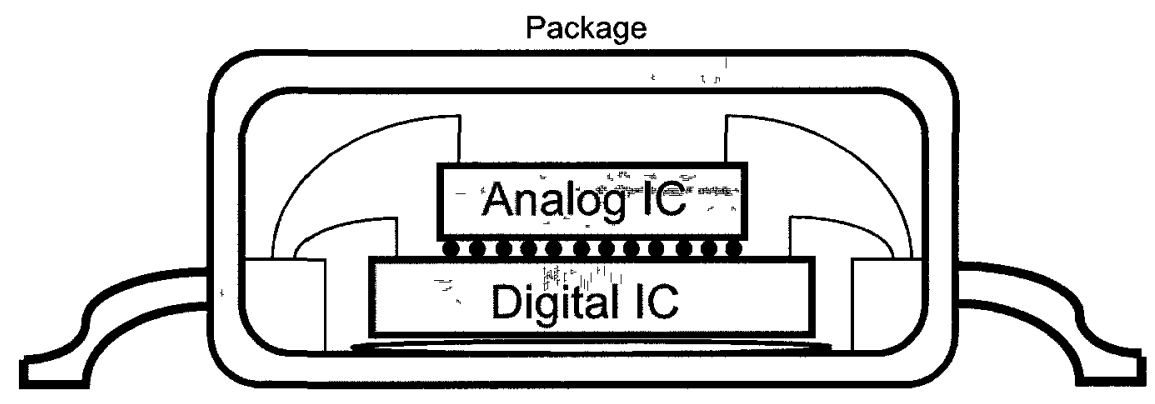

Figure 2.2: The SiP approach to system integration combines multiple specialized ICs in one package.

Necessary interconnects can be incorporated into the supporting package. Other advantages over SoC integration include i) simpler design and verification, ii) less complex fabrication, and iii) fewer IP issues. An obvious disadvantage of the SiP approach is higher complexity associated with packaging and reliability. A critical drawback of SiP integration is the fact that it is limited by IC technology [11]. If a particular feature is required by the system such as high $\mathrm{Q}$ passives, a $\mathrm{SiP}$ approach is likely to fail because IC technology is not well suited to meet this requirement. Due to these problems, $\mathrm{SiP}$ integration may not be the best solution for many demanding wireless applications of the future.

As we have just seen, neither the SoC nor SiP approaches represent ideal solutions to the problem of system integration. SoP is the newest attempt to improve upon the shortcomings of both SoC and SiP while achieving the highest system performance 
at the lowest cost. SoP introduces a revolutionary concept that basically changes the definition of a "package". For SoC and SiP designers, the "package" can be defined as a contaner for ICs that form a system when they are combined with a myriad of discrete components on a Printed Circuit Board (PCB). But in SoP, the PCB per-se no longer exists and the package provides all the system functions in one module [13]. In SoP, the package supports multiple vertically and horizontally stacked ICs as well as housing embedded high-quality passives, routing, and even additional active electronics [14]. Fig. 2.3 shows this concept graphically.

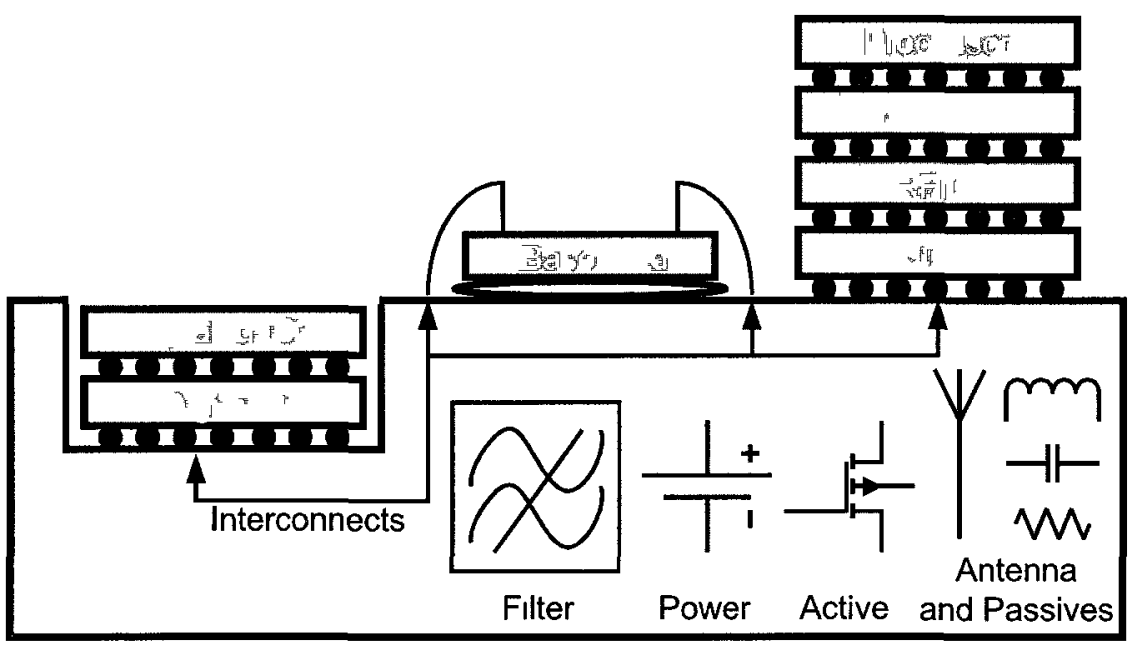

Figure 2.3: The SoP approach to system integration combines multiple specialized ICs with an advanced package that also provides many system functions.

This marriage of different subsystems is accomplished through the use of thin film technologies and the co-design of all the ICs and the package [15]. SoP can claim to provide better system performance than either SoC or SoP because it uses CMOS and the package in the best way [14]. That is, CMOS is used for transistor integration and the package for passives and interconnect integration. The availability of low loss packaging technologies lıke LTCC and thin film, are major enabling factors in the 
success of SoP integration. Other necessities that exist today are the availability of cheap yet powerful computer resources and full three-dimensional electromagnetic solvers. These resources and tools are needed to account for significant element coupling, parasitic effects, and thermal issues that are associated with complex SoP designs. Due to the relative immaturity of SoP technology, at least one outstanding issue exists - the development of a hierarchical design flow like the one that is so prevalent in the SoC realm.

In this section the relative strengths and weaknesses of three system integration methods: SoC, SiP, and SoP have been described in some detail, while Table 2.1 summarizes them. Of these three, it is SoP that holds the most promise of fulfilling the requirements of present and future communication systems. Thus, it has been chosen as the integration method to implement the work of this thesis.

Table 2.1: System Integration Methods Overview

\begin{tabular}{|c|c|c|c|}
\hline & Description & Strengths & Weaknesses \\
\hline $\mathrm{SoC}$ & $\begin{array}{l}\text { All functionality in- } \\
\text { tegrated on chip }\end{array}$ & $\begin{array}{l}\text { Potential for lowest } \\
\text { cost }\end{array}$ & $\begin{array}{l}\text { Advanced fabrication } \\
\text { requirement }\end{array}$ \\
\hline $\mathrm{SiP}$ & $\begin{array}{l}\text { Functionality divided } \\
\text { among separate chips } \\
\text { arranged vertically or } \\
\text { horizontally }\end{array}$ & Simpler design & $\begin{array}{l}\text { Still limited by } \mathrm{IC} \\
\text { technology }\end{array}$ \\
\hline SoP & $\begin{array}{l}\text { Package provides ad- } \\
\text { ditional functionality }\end{array}$ & Best performance & Difficult to design \\
\hline
\end{tabular}




\subsection{Technology Medium}

To implement the SoP approach, a substrate material must have many important attributes such as low loss, multilayer capability, high reliability, and so on. Today, the two leading substrate materials that are best suited for SoP designs are LTCC and Liquid Crystal Polymer (LCP). The former is a very mature technology whose first reported RF application occurred in the early 1990's [16]. On the other hand, LCP is relatively new, having only been fully characterized in 2004 [17]. Despite this difference in age, these materials offer similar benefits. However, the fact that LTCC is a ceramic material and LCP is an organic polymer lead to some important differences. A comparison of the two materials is given in Table 2.2.

Table 2.2: A Comparison of Material Systems

\begin{tabular}{|l|l|l|}
\hline Item & LTCC & LCP \\
\hline $\begin{array}{l}\text { Dielectric Con- } \\
\text { stant }\end{array}$ & $\begin{array}{l}4-8,13-14,16,17, \\
65,18-250 \text { (capacitor } \\
\text { tape) }\end{array}$ & $2.95-4.4$ \\
\hline $\begin{array}{l}\text { Relative Perme- } \\
\text { ability }\end{array}$ & $\begin{array}{l}1,60,200,450 \text { (fer- } \\
\text { rite) }\end{array}$ & 1 \\
\hline Loss Tangent & $<0.002$ & $<0.002$ \\
\hline $\begin{array}{l}\text { Maximum No. of } \\
\text { Layers }\end{array}$ & $60+$ & $4+$ \\
\hline Frequency Range & 0 to $110 \mathrm{GHz}$ & 0 to $110 \mathrm{GHz}$ \\
\hline Water Absorption & $N / A$ & $<8 \%$ \\
\hline Relative Cost & medium & low \\
\hline
\end{tabular}

In terms of electrical performance, reliability, and cost, these two materials are very similar. The most important differentiating factor for SoP designers is that 
LTCC offers a range of different dielectric constants and even the capability to create heterogenous ferrite substrates [18], while LCP is limited to only a small range of homogeneous examples. Also, LTCC allows for many more layers to be stacked than LCP. These critical advantages make LTCC a better choice as an SoP technology for this work.

\subsection{System Overview}

The system architecture proposed here utilizes a conventional receiver chain that includes an antenna, BPF, LNA, hybrid coupler, and a quadrature downconverter with in-phase (I) and quadrature (Q) outputs. Each component is designed for a 50 Ohm system impedance. The architecture is presented with the help of a block diagram in Fig. 2.4.

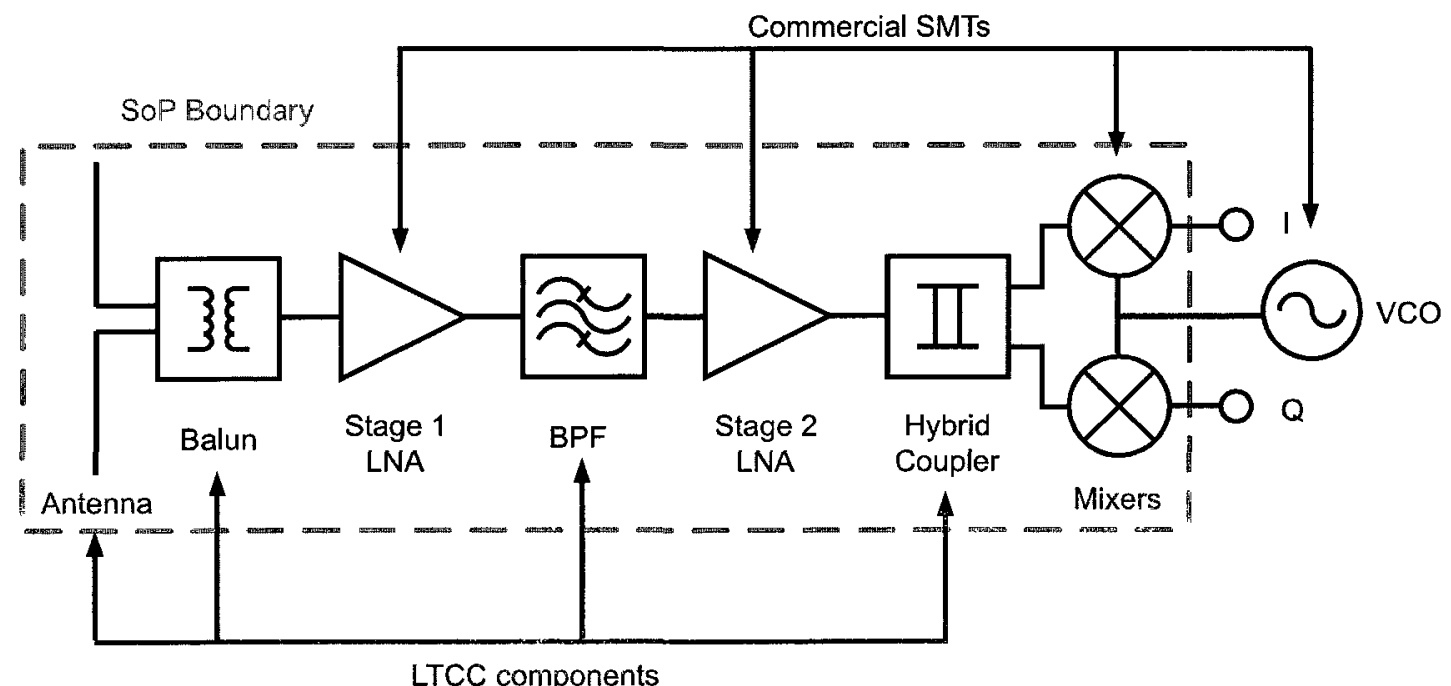

Figure 2.4: Proposed system architecture.

The boundary of the SoP module is indicated with a dotted line. All components 
within this boundary are either package embedded within the module or mounted on top of it. The module size is chosen to be that of a Secure Digital (SD) memory card, which has dimensions of $32 \times 24 \times 2.5 \mathrm{~mm}^{3}$. Given the SD card's widespread use, a GPS module that conforms to this standard could be easily marketed. To the best of the author's knowledge, this is the smallest GPS receiver front-end module ever reported. The material system chosen to implement this module is FerroA6 - a low loss LTCC ceramic. Table 2.3 lists the material properties of FerroA6.

Table 2.3: LTCC Material Properties

\begin{tabular}{|c|c|}
\hline \multirow{4}{*}{ Substrate } & FerroA6 \\
& $\varepsilon_{r}=5.7$ \\
& $\tan \delta=0.002$ \\
& Height $=0.72 \mathrm{~mm}$ (8 layers) \\
\hline \multirow{3}{*}{ Conductor } & Gold \\
& Thickness $=7 \mu \mathrm{m}$ \\
& Bulk Conductivity $=4.5 \times 10^{7} \mathrm{~S} / \mathrm{m}$ \\
\hline
\end{tabular}

The antenna is the limiting factor in the miniaturization of the module but a sufficiently compact design is presented in Chapter 7 . The next element in the receiver chain is the bandpass filter. This element is embedded in the substrate also to reduce the size and cost of the module. The primary challenge associated with the design of the bandpass filter will be to simultaneously achieve low in-band insertion loss and good stop-band rejection. The LNA is implemented with a commercial SMT component and soldered to the top of the module. Embedded interconnects link this amplifier to its adjacent components. As with the bandpass filter, the hybrid coupler is embedded in the substrate to provide similar benefits in terms of module size and 
cost. Its purpose in this work is to provide quadrature signals for the downconverter and later on for image rejection.

\subsection{Bandpass Filter Background}

A bandpass filter is an essential building block of any wireless communications system. Furthermore, filters with high stopband attenuation effectively reduce interference from strong jamming signals and as a result improve the signal-to-noise ratio in the receiver. The GPS signal is especially susceptible to jamming because of its low signal strength (approximately $-127 \mathrm{dBm}$ ) after traveling for thousands of kilometers from space [19]. Thus, this class of filter with high stop-band rejection is a desirable component of a good quality receiver. To effectively block strong interferer signals, an attenuation of more than $50 \mathrm{~dB}$ is recommended. This remains an area where improvements can be made since previous work has not achieved this level of attenuation over a broad stopband [20-22].

The image parameter method and the insertion loss method are two major design methods that result in lumped element circuits [23]. For their implementation two categories exist: i) the distributed element method, which includes the use of stepped impedance transformers and coupled lines and ii) the lumped element approach. At microwave frequencies and space permitting, the distributed element implementation is usually preferred. However, at lower microwave frequencies and where space is at a premium the lumped element approach is often chosen. GPS as an application falls into the latter category.

Ideally, the bandpass filter should be compact, planar, low-cost, and reliable. The 
primary challenge involved in designing an L-band GPS filter is successfully realizing the very narrow fractional bandwidth, typically less than $5 \%$. The traditional solution has been to use bulky acoustic wave filters [24], [25], or combline filters [26]. Recently, lumped element LTCC filters have been designed for applications other than GPS [21,22,27]. Although compact, they have unsuitable fractional bandwidths and insertion losses. Other work has focused on using distributed elements to create coupled resonator filters $[28,29]$. However, these designs exhibit comparatively large sizes due to the fundamental disadvantage of using quarter-wave elements at relatively low frequencies. Also, these designs resort to unconventional high permittivity substrates to reduce the size of the filters. The lumped element approach represents a simpler solution that is intuitive and realizable due to the availability of low loss multilayer substrates and well known equivalent models (Fig. 2.5 [6]).

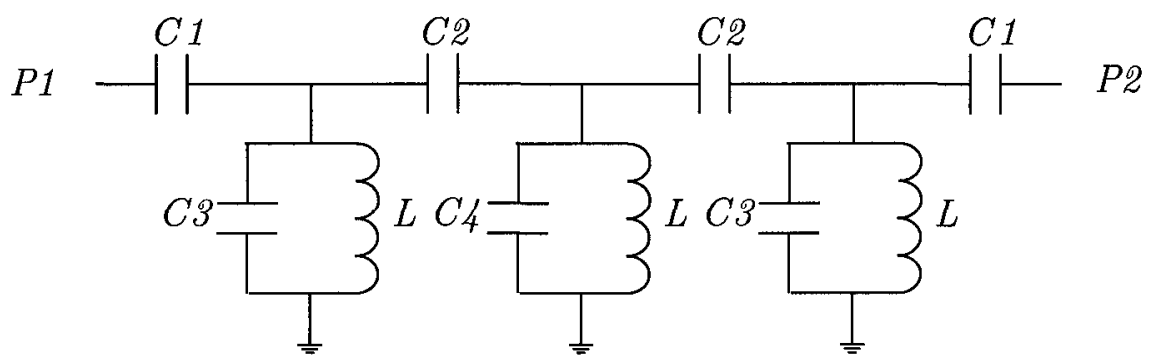

Figure 2.5: Lumped element schematic of bandpass filter.

Stopband rejection can be increased by raising the order of the filter. The tradeoff is an increase in filter complexity, size, and passband insertion loss. The ability to create multilayer embedded components in LTCC has succeeded in offsetting the size penalty of more complex filters. But herein lies another tradeoff caused by highly compact topologies: optimization becomes increasingly difficult due to the fact that circuit element values can become electromagnetically coupled to each other. For 
example, placing two inductors in close proximity will create some degree of mutual coupling that can be detrimental to the performance of the circuit.

The key to meeting the size and performance requirements is a high-Q L-C resonator structure. Such a resonator can be created by the parallel combination of a multilayer capacitor and a high-Q inductor. Designing a bandpass filter based on this structure in a very low loss substrate such as LTCC promises to simultaneously satisfy these requirements.

\subsection{Microwave Coupler Background}

Hybrid couplers belong to an important category of microwave passive devices that includes power dividers and directional couplers. Power dividers have at minimum three ports and can divide an input signal by some arbitrary fractional amount. Directional couplers commonly have four ports and can also divide an input signal. In addition, they can also introduce a finite phase shift between the output ports. The phase shift can be made to be 90 degrees in which case the device is called a quadrature hybrid coupler. On the other hand, if the phase shift is made to be 180 degrees then the device is called a magic- $T$ or balun.

In this work, both types of couplers are proposed to perform critical functions. The 90-degree hybrid coupler is used to divide an RF signal before it is applied to an image reject mixer, while the 180 coupler acts as a balun for the differential dipole antenna discussed in the next section. Usually, couplers are realized in some type of microstrip or stripline technology [23] [30]. However, at L-band frequencies, a conventional microstrip coupler using distributed quarter-wave sections would require several $\mathrm{cm}^{2}$ of area to be realized. Even clever miniaturization techniques such as meandered and 
folded lines only achieve a maximum size reduction of $63 \%$ [31] [21] compared to the conventional approach. Naturally, this high area requirement excludes this type of coupler from being a solution for miniature GPS devices. Other work has shown a size reduction of $80 \%$ but has the cost disadvantage of using surface mount components [32]. Even novel transmission line techniques such as composite right/left-handed lines and slow-waves give size reductions of $55 \%$ and $86 \%$, respectively [33,34]. A lumped element approach represents a simpler solution that is very feasible due to the availability of low loss multilayer substrates and lumped element equivalent models [2]. These models, shown in Fig. 2.6 can be realized with very small passive components and yield a coupler that has a degree of miniaturization above $90 \%$ [8].

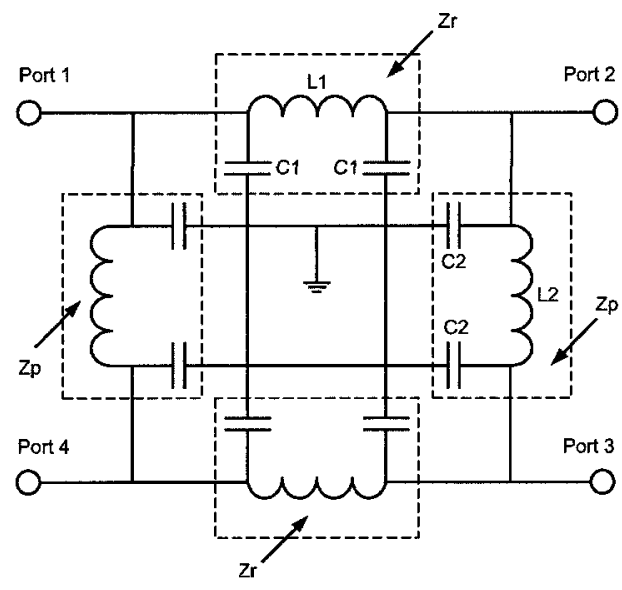

(a)

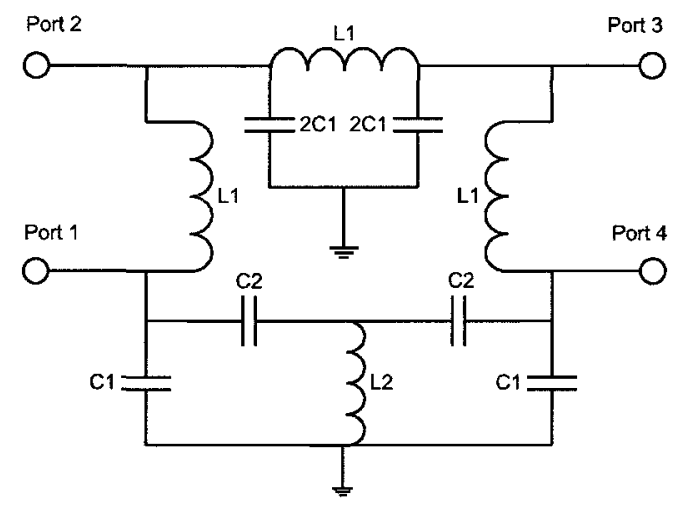

(b)

Figure 2.6: Symmetric lumped element equivalent models: (a) 90-degree hybrid coupler and; (b) 180-degree coupler.

Therefore, just as in the case of the bandpass filter in the previous section, using a lumped element approach can succeed in miniaturizing the device substantially. Also, since the equivalent lumped element model of a coupler only includes nonresonant pi networks, the insertion loss of the device is less sensitive to the Q-factor 
of the inductors used. The same challenge remains, however, to account for parasitic coupling of all the elements that are very closely spaced.

\subsection{Antenna Element Background}

Even with the design freedom afforded by SoP technology, the task of successfully integrating an antenna is a difficult one because of the limited space available. The antenna may either be integrated on a chip or embedded in the package. The on-chip approach results in the smallest antenna size, however there are many well-known disadvantages. Primarily, the radiation efficiency is severely degraded because of the lossy nature of the silicon substrate and this can limit the communication range to below $2 \mathrm{~m}$ for low power applications like Radio Frequency Identification (RFID) $[35,36]$. Secondly, the antenna itself may occupy a significant portion of the on-chip area that may have been otherwise used by transistors [37,38]. Finally, in some applications such as GPS, the operating frequency is so low that integration on-chip becomes physically and financially impractical. Therefore, embedding the antenna in a low-loss package becomes the best solution. A package antenna can improve the radiation performance substantially, while at the same time freeing up on-chip space.

Not every type of antenna element is suitable for SoP integration. Most importantly, the element should be planar so as to occupy as few substrate layers as possible. Also, it should lend itself to common miniaturization techniques such as meandering and folding to minimize its size. By virtue of being housed in a small package, the element should not be dependent on a large ground plane. An omnidirectional radiation pattern is preferred, especially for a receiver whose orientation in space may be arbitrary. Finally, some degree of tunability (whether electronic or manual) would be 
a distinct advantage. The antenna elements considered here are shown in Fig. 2.7.

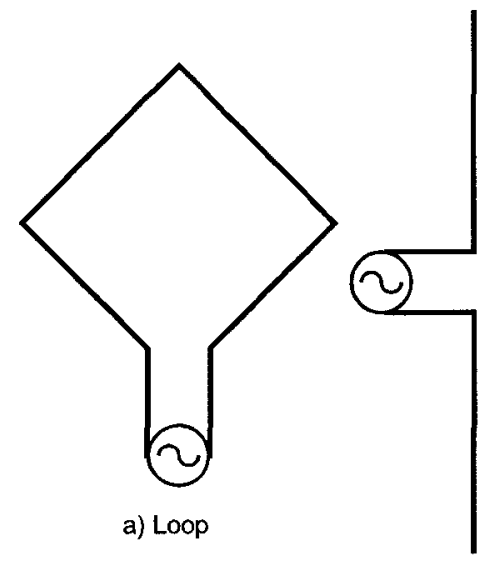

b) Dipole

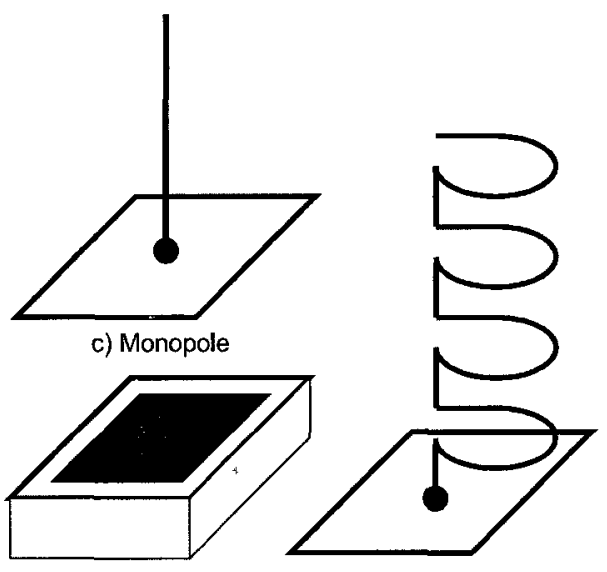

d) Patch

e) Spiral/helix

Figure 2.7: A survey of the antenna elements considered here for a GPS SoP.

Since its inception in the 1980s, the GPS standard has been used primarily for military and commercial applications where device size was not an important consideration. Now, system integrators and hand-set makers have realized the value-added potential of GPS and are including it in their consumer products. However, because of the recent transition to consumer products, very little research work has been conducted in the area of small GPS antennas [39]. Therefore, a study of antennas for general mobile communications systems will be conducted with a focus on how they may be adapted for GPS applications.

It must be mentioned that the GPS signal is Right Hand Circularly Polarized (RHCP) and so a similarly polarized antenna would theoretically give the best reception, while a linearly polarized antenna would only receive half the available power. However, studies have shown that linearly polarized antennas can provide performance similar to that of more complex RHCP antennas [40]. Furthermore, in the case of a rich multi-path environment (as considered here), a linearly polarized 
antenna may be preferred since it is less susceptible to distortion caused by the environment and by the presence of the user [41]. Therefore, this study will include both types of antennas as candidates for integration in a SoP receiver.

Since the availability of unlicensed spectrums around $2.4 \mathrm{GHz}$ and $5.8 \mathrm{GHz}$, general communications systems have been defined by the popularity of Wireless Local Area Networks (WLANs). The $802.11 \mathrm{a} / \mathrm{b} / \mathrm{g}$ standards have been written to work in these bands and have thus defined the design criteria for compatible antennas. Many WLAN applications require a small antenna that can be easily placed inside a personal communication device such as a cellular phone or a handheld computer. The most common type of antenna employed is the so called Planar Inverted-F Antenna (PIFA) element because of its compact size and low profile. A vast amount of work has been published based on variations of the original concept [42-49]. These antennas work well but are designed to operate with a ground plane whose size has a significant impact on resonant frequency, gain, radiation patterns, and cross-polarisation [50,51]. In this work, the ground plane is assumed to be very small $(<0.2 \lambda)$, which makes the PIFA antenna a poor candidate.

Microstrip patch antennas are extensively used for GPS applications because of their good gain characteristics and ability to radiate linear or circularly polarized fields [52-55]. They often employ expensive high permittivity substrates for miniaturization with the trade-off being a reduction in bandwidth that can be improved by stacking multiple patches. Nonetheless, these compromises lead to thick and still relatively large antennas. Furthermore, these designs are hampered by the requirement for a ground plane that may be as large as $196 \mathrm{~cm}^{2}$ [56]. Ultimately, for these reasons, a microstrip antenna cannot be miniaturized to the degree required here. Other antennas that may be discounted are the helix and spiral topologies because of 
their large size and non-planar profile [57]. Two elements that avoid these problems are the monopole and dipole, which are both widely used in wireless communications. Compared to the monopole, the dipole has two important advantages: i) orthogonal ground plane not required and ii) input impedance is doubled [58]. Furthermore, the dipole is a differential element that has greater immunity to common mode noise. By using meandering lines, the dipole may be miniaturized relatively easily and electronic tunability may be implemented to account for ground plane effects [59-61]. Another related element is the loop antenna. It is similar to the dipole by virtue of its omnidirectional radiation pattern and differential input. However, this element suffers from poor radiation efficiency due to the fact that its radiation resistance is smaller than its loss resistance and a pronounced susceptibility to ground plane effects [58]. A survey of the relative properties of these antenna elements is shown in Table 2.4.

Table 2.4: Antenna Element Survey

\begin{tabular}{|l|c|c|c|c|c|}
\hline & Loop & Dipole & Monopole & Patch & Spiral $\backslash$ Helix \\
\hline \hline Differential? & Yes & yes & No & No & No \\
\hline Planar? & Yes & Yes & No & Yes & No \\
\hline Tunable? & Not easily & Yes & Not easily & Not easily & Not easily \\
\hline $\begin{array}{l}\text { Suitable for } \\
\text { miniaturiza- } \\
\text { tion? }\end{array}$ & Yes & Yes & No & Not easily & No \\
\hline $\begin{array}{l}\text { Omnidirectional } \\
\text { radiation? }\end{array}$ & Yes & Yes & No & No & Yes \\
\hline $\begin{array}{l}\text { Susceptibility } \\
\text { to ground } \\
\text { plane effects? }\end{array}$ & Large & Small & Yes & Yes & Yes \\
\hline
\end{tabular}

Therefore, it has been shown that a small dipole antenna is the best option for 
integration in a SoP receiver for GPS applications. The main challenge will be to design the antenna so that its performance is not severely impacted by the other embedded components in the SoP device.

\subsection{Downconverter and Image Reject Mixer Back- ground}

In heterodyne (and superheterodyne) receivers the signal band (RF) is downconverted to a lower Intermediate Frequency (IF) to reduce the $\mathrm{Q}$ requirements of the channel select filter. This is accomplished by multiplying the RF signal by a Local Oscillator (LO) frequency so that $f_{L O}=f_{R F}-f_{I F}$. Subsequent demodulation occurs at the IF frequency. The GPS standard requires Binary Phase-Shift Keying (BPSK) modulation for transmitted signals. Therefore, a quadrature downconverter must be used to avoid loss of information. The 90-degree phase shift can be created by means of a passive polyphase filter [62] or a hybrid coupler [63]. Process variations associated with the resistors and capacitors can severely reduce the performance of polyphase filters [62], while hybrid couplers may only be feasible at high frequencies where their size is reduced [63]. A newer approach forgoes the use of passive components for an active component. Namely, a Quadrature Voltage Controlled Oscillator (QVCO), which automatically generates two signals that are separated by 90 -degrees [64]. In this work, however, an LO signal will be provided externally so a QVCO becomes redundant.

Unfortunately, in heterodyne receivers, an undesired band that is symmetrically located on the other side of the LO frequency $\left(f_{L O}=f_{R F}+f_{I F}\right.$ in this case) is also downconverted. This undesired band is commonly called the image and presents a 
serious problem due to the fact that it is downconverted on top of the desired signal. This can cause the destruction of the message if the image is a relatively high power signal. Suppressing the image can be achieved by including an image reject filter, using a dual-IF or zero-IF architecture, or employing an Image Reject Mixer (IRM). The first option typically places a filter before the mixer that has low insertion loss at the RF frequency but high attenuation at the image frequency. The filter can be either of a bandpass type [22] or a notch type $[65,66]$. This can potentially represent the simplest solution. However, there is a trade-off between sensitivity and selectivity because of the choice of IF frequency and filter Q limitations [67]. Furthermore, as an extra component, the filter will occupy more space or consume more power (if it is an active implementation). Therefore, if these tradeoffs are too severe then a dual-IF or zero-IF architecture may be adopted. These architectures, however clever, introduce a large amount of complexity to the receiver and undesired disadvantages such as $I / Q$ mismatch and even order-distortion [67].

Employing an IRM represents an attractive compromise between the complexity of dual-IF or zero-IF architectures and the sensitivity-selectivity tradeoff of image filters. An IRM works by adding a negated duplicate of the image to itself. In the case of a quadrature downconverter, this only necessitates the addition of one 90degree phase shift block. The cancelation depends on the accurate introduction of a 90-degree phase shift to one of the LO or IF branches. This work forms part of a receiver architecture that incorporates an IRM. Further image rejection is performed in the digital domain by a microprocessor, which is beyond the scope of this work. The receiver front-end designed here will be tested by inserting it into the receiver chain of an existing SkyWave Inc. device (and disabling its front-end). This will provide a very accurate comparison of absolute and relative performance. 


\subsection{Summary}

This chapter has described the benefits and challenges of adopting an SoP approach to the design of a L-band receiver. To implement this design, an LTCC material system is chosen based on its maturity and versatility. GPS is chosen as the vehicle to demonstrate advanced packaging techniques and novel system components. A literature review is presented for the bandpass filter, coupler, downconverter, balun, and antenna. A dipole element is chosen because it is best suited to the environment that this receiver is likely to be placed in. Given this basis, the next chapters describe the design of each of these components and the complete module. 


\section{Chapter 3}

\section{BPF Design and Methodology}

In accordance with the second thesis objective, this chapter presents a novel methodology used to design a bandpass filter embedded in a LTCC package. Here, the purpose of the bandpass filter is to attenuate out-of-band interferer signals and its position in the receiver chain is presented in Fig. 3.1.

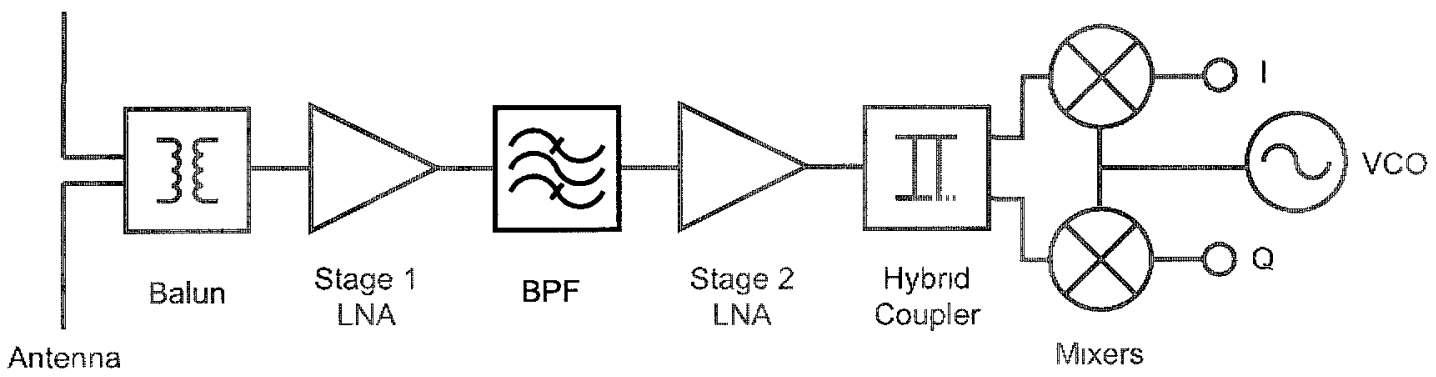

Figure 3.1: Bandpass filter as part of the complete receiver chain.

Given the three-dimensional packaging capability of LTCC, multilayer resonators are integrated into the module eliminating the need for their discrete versions. The resonators are comprised of high $\mathrm{Q}$ spiral inductors that are combined with four layer parallel plate capacitors, forming a novel L-C resonator topology. This topology of placing the capacitor in the center and two layers below the plane of the inductor 
allows for simultaneous area and performance optimization. The final LTCC bandpass filter module measures only $5 \mathrm{~mm} \times 5.4 \mathrm{~mm} \times 0.8 \mathrm{~mm}$. The complete methodology, from initial schematic design, through individual element design, to complete device optimization is discussed.

\subsection{Overview of Methodology}

A novel methodology for the design of highly integrated multilayer embedded components that are mutually coupled is proposed. The methodology relies on the ability to replace, in simulations, some embedded components with ideal impedance boundaries whose values are fixed and immune to mutual coupling. Current industry software tools such as Ansoft's High Frequency Structure Simulator (HFSS) have the capability to support this methodology. Using our technique, the designer can employ a "divide and conquer" approach to LTCC circuit design. The potential benefits are a better understanding of the limitations of equivalent circuit models and a reduction in the time required for design and optimization.

The three filters designed and characterized herein show the transition from a partially to fully embedded design. Fig. 3.2 shows a three-dimensional rendering of one of the proposed filters (with internal metal layers visible) and its equivalent circuit schematic.

\subsection{LTCC Design Enhancement Methodology}

Highly miniature three-dimensional LTCC modules are becoming increasingly difficult to design and more demanding to simulate. Two significant causes for this are 


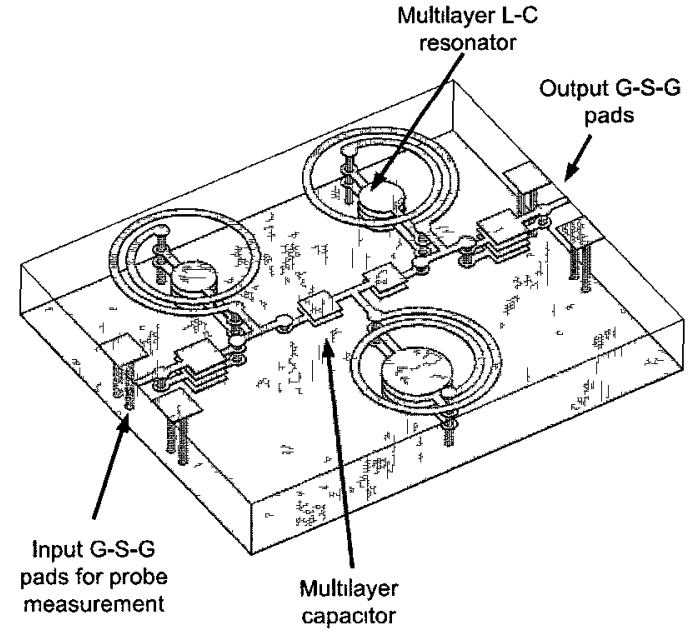

(a)

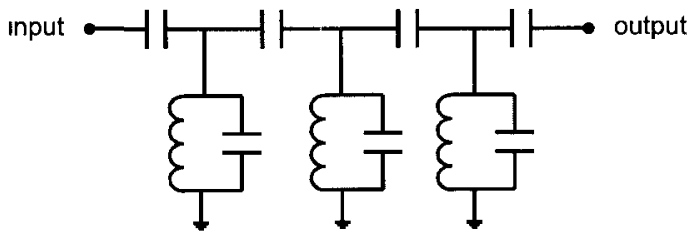

(b)

Figure 3.2: Bandpass filter (a) three dimensional structure and; (b) its equivalent schematic.

parasitic effects and mutual coupling of passive structures. Therefore, to ensure design success these effects must be fully understood and accounted for. Traditional approaches create equivalent pi or T models to capture this behavior. Unfortunately, such models are only suitable for small two-terminal devices. When used to model multilayer embedded structures they become very complex and cumbersome [68].

A new methodology is proposed that does not require formulating complex equivalent models. Rather, it takes advantage of low cost, yet powerful computer resources and mature electromagnetic (EM) simulators. Many mature software packages exist. In this work, Ansoft HFSS was chosen as the tool to perform full three-dimensional EM simulations. HFSS has an important feature that permits the incorporation of ideal components in full three-dimensional EM simulations. This feature allows for the creation of "hybrid" EM models, i.e. intermediate structures that are a combination of ideal and real elements. This is significant because it simplifies the design of 
large LTCC modules by replacing one large difficult design task with smaller, more manageable ones.

The design process is iterative and begins with a hybrid EM structure and finishes with a fully real structure whose parasitics and mutual coupling attributes are fully understood and accounted for. An advantage of this technique is that it reduces the time and effort required to create an optimized design since there is no need to develop large equivalent models.

In this methodology, the first step is to determine a simple equivalent circuit for the entire LTCC structure. In this case, a bandpass filter is chosen whose design specifications and circuit schematic are given in Section 3.3. A multilayer structure can be created for each individual inductor and capacitor that corresponds to a lumped element representation. They are then simulated in HFSS and adjusted to provide the exact capacitance or inductance required.

At this point, combining these capacitor and inductor structures to form a complete filter will likely result in a much different response than expected. And re-tuning would be difficult because of many design variables involved. A more efficient approach is to first create a hybrid structure that replaces all four coupling capacitors with ideal impedance boundaries that are set to the required capacitances (see Fig. $3.3[6])$. In this way the complexity of the structure is reduced and some parasitic and coupling effects are eliminated. Now, this filter's performance can be tuned more easily to give the ideal response. Fig. 3.4 shows a comparison between the design flow for the newly proposed and conventional methodologies. It shows that the new methodology replaces one difficult tuning step with two easier ones. In this way the overall time spent optimizing can be substantially reduced.

Once the filter's response is satisfactory a pair of coupling capacitors, which were 


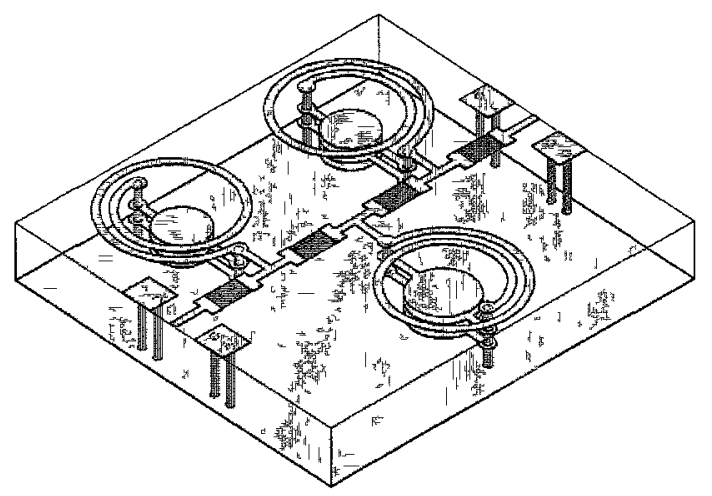

Figure 3.3: Lumped element bandpass filter structure with four ideal capacitors replaced in the final implementation.

previously modeled, can replace their ideal counterparts. Fig. 3.5 shows a modified hybrid structure that has had its inner ideal coupling capacitors replaced with real structures.

Simulating the new structure will most likely reveal a degraded filter response because of the newly introduced parasitic and coupling effects. However, now the cause of these effects is clear and can be adjusted through the fine tuning of the newly introduced capacitor geometries. A simple circuit simulator is useful here since it can indicate whether the capacitance needs to be increased or decreased based upon the differences between the EM and circuit simulator response. Performing the needed number of iterations of this procedure will result in the replacement of all the ideal boundaries with real structures to form a fully embedded LTCC module, as shown in Fig. 3.2(a).

It should be noted that Model Order Reduction (MOR) techniques have been extensively developed to reduce simulation times [69] [70]. MOR techniques rely on the use of specialized mathematical formulations and macromodels of circuits $[71,72]$. Unfortunately, many MOR techniques have practical limitations and only 


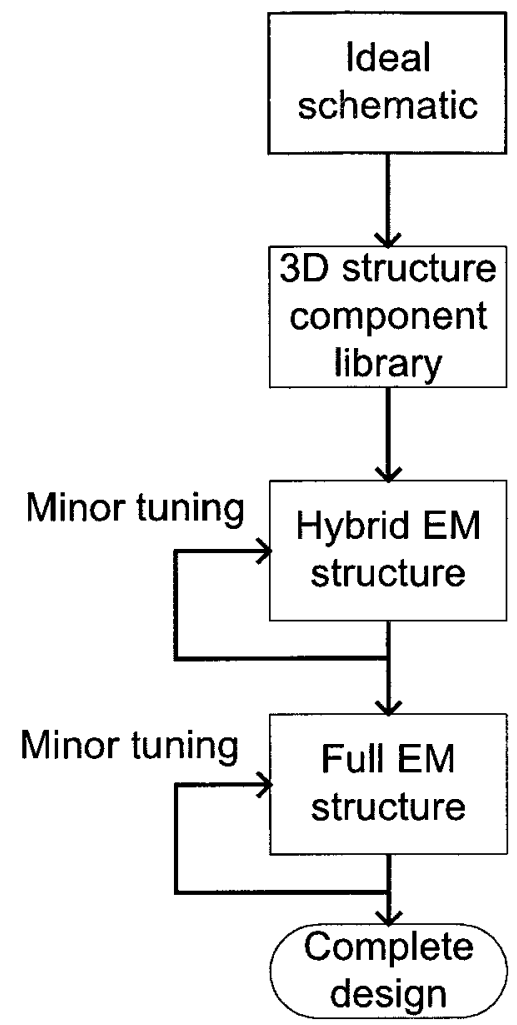

(a)

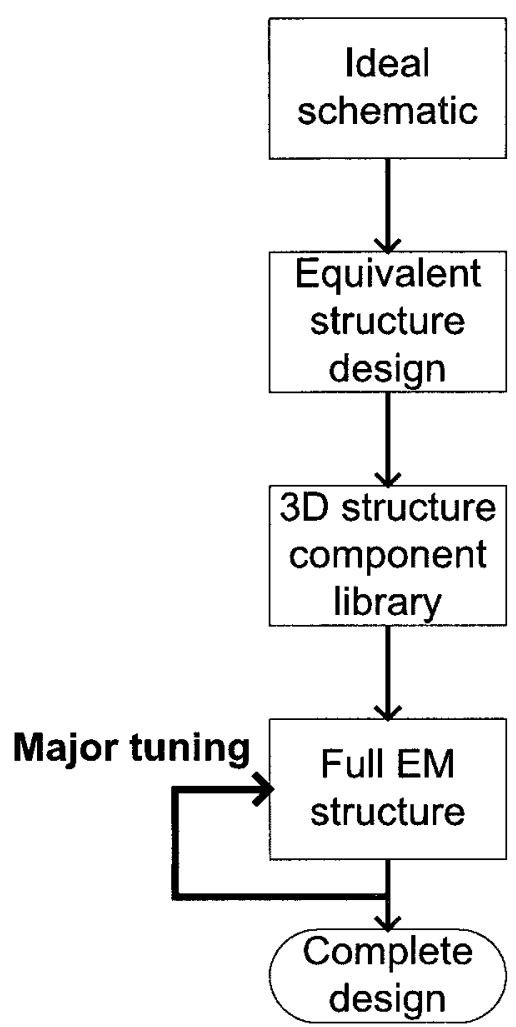

(b)

Figure 3.4: Flow chart comparison of (a) the proposed design methodology, and (b) the conventional approach.

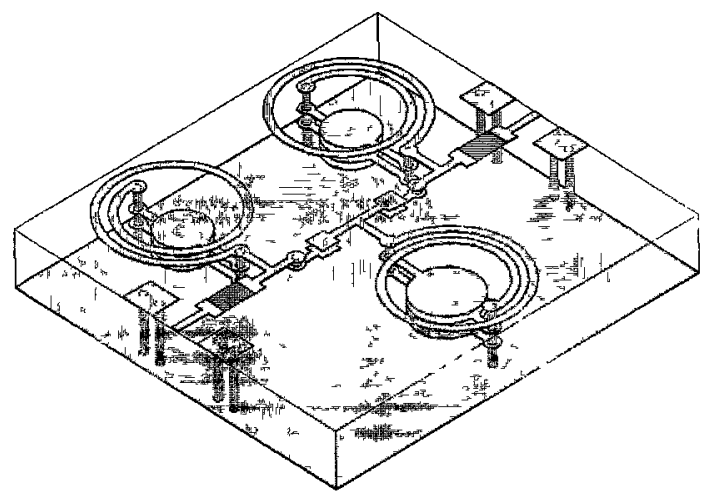

Figure 3.5: Lumped element bandpass filter structure with two idcal coupling capacitors. 
achieve significant benefits in ideal circumstances [73]. This work does not rely on any such complicated and specialized techniques. Therefore, it can only be considered as a partial MOR scheme, in that some real elements are replaced by simpler ideal equivalents. Furthermore, the ultimate goal of this approach is not to reduce the circuit complexity but rather to improve the filter design process through greater insight into EM coupling effects.

\subsection{Design Specifications and Circuit Schematic}

A design study based upon a third-order lumped element bandpass filter was chosen to validate the methodology described above. The filter is based on a Chebyshev approximation of a capacitively coupled, lumped element topology and has the schematic shown in Fig. 3.6 [6]. This design offers relatively low insertion loss in the pass-band, while providing acceptably high attenuation out of band. The filter is designed to

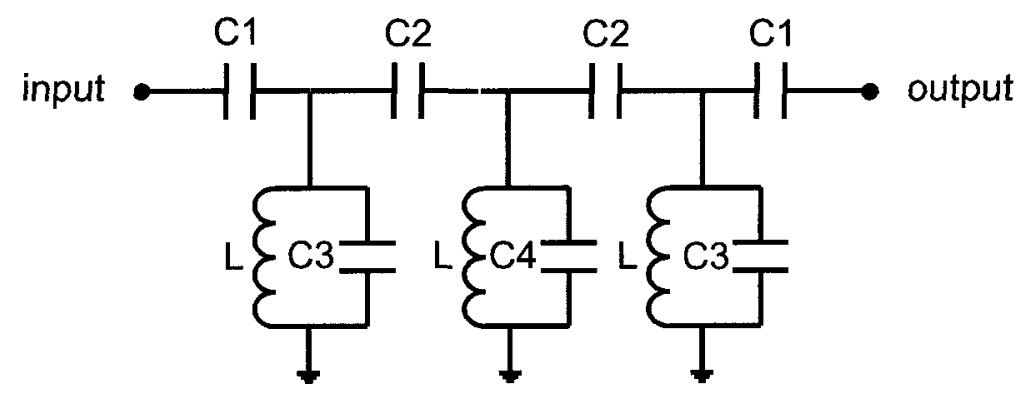

Figure 3.6: Lumped element schematic of bandpass filter.

have a center frequency of $1.55 \mathrm{GHz}$, a $10 \mathrm{~dB}$ bandwidth of $100 \mathrm{MHz}$, an insertion loss in the passband not exceeding $2.5 \mathrm{~dB}$, an in-band return loss of $10 \mathrm{~dB}$ and finally, a stop-band insertion loss greater than $20 \mathrm{~dB}$ at a frequency offset of $150 \mathrm{MHz}$ from the center frequency. 
Three filters based on these specifications were designed and characterized. Their designs differ by the number of surface mount coupling capacitors employed. The first design (Fig. 3.3) has four capacitors, the second design (Fig. 3.5) has two, while the last design (Fig. 3.2(a)) has none and represents a fully embedded filter structure.

All these filters share one common component - the spiral inductor that forms part of each L-C resonator. Being the largest component, special consideration was reserved for this part. First, an in-line geometry was required to facilitate capacitor integration. This limited the possible number of turns to be $0.5,1.5,2.5$ and so on. 1.5 turns resulted in the best solution that simultaneously achieved a high Q-factor with a useful amount of inductance. Section IV discusses the procedure that was used to obtain the values of $7.1 \mathrm{nH}$ and 86 as the inductance and Q-factor, respectively. A line width and separation distance of $100 \mu \mathrm{m}$ was chosen based on the guidelines in $[74]$.

Using the inductor parameters, initial component values were obtained. Standard values for $C 1$ and $C 2$ were found to be $0.5 \mathrm{pF}$ and $0.1 \mathrm{pF}$, respectively. Now, the schematic was optimized with these new component values and the results are contained in Table 3.1.

Table 3.1: Theoretical Component Values For Bandpass Filters

\begin{tabular}{|c|c|}
\hline Component & Value \\
\hline$L$ & Ind. $=7.1 \mathrm{nH}$, Q-factor $=86,1.5$ turns \\
\hline$C 1$ & $0.5 \mathrm{pF}$ \\
\hline$C 2$ & $0.1 \mathrm{pI}$ \\
\hline$C 3$ & $1.1 \mathrm{pF}$ \\
\hline$C 4$ & $1.44 \mathrm{pF}$ \\
\hline
\end{tabular}




\subsection{Passive Component Design}

LTCC technology allows for the miniaturization of filter designs because of the possibility to stack layers and embed passive components. Also, it has been proven to be a low loss microwave substrate suitable for a wide range of applications, including antennas [75]. These advantageous features imply that designs created on similar material systems can be combined to create system-in-package designs. Table 3.2 presents the properties of the material system employed in this work. In the follow-

Table 3.2: LTCC Material Properties

\begin{tabular}{|c|c|}
\hline Substrate & $\begin{array}{c}\text { FerroA6 } \\
\varepsilon_{r}=5.9 \\
\tan \delta=0.002 \\
\text { Height }=0.8 \mathrm{~mm} \text { (8 layers) }\end{array}$ \\
\hline Conductor & $\begin{array}{c}\text { Silver } \\
\text { Thickness }=13 \mu \mathrm{m} \\
\text { Bulk Conductivity }=6.1 \times 10^{7} \mathrm{~S} / \mathrm{m}\end{array}$ \\
\hline
\end{tabular}

ing subsections the necessary inductors, capacitors, and resonators are designed. This collection of structures represents the second stage in the design process, namely, the creation of a component library that will be used to assemble the filters.

\subsubsection{Circular Spiral Inductor Design}

The inductor Q-factor has a strong influence on a filter's in-band insertion loss [74]. Therefore, some well established inductor design guidelines were followed to reduce losses [76]. This resulted in selecting a planar spiral topology with diameter of $1.8 \mathrm{~mm}$. Furthermore, the inductor was placed on the top metal layer to reduce dielectric losses. 
The number of turns was selected by studying the relationship between inductance and Q-factor. This is depicted in Fig. 3.7, where the data is obtained from the simulation of four inductor structures with $0.5,1.5,2.5$, and 3.5 turns. It's desirable

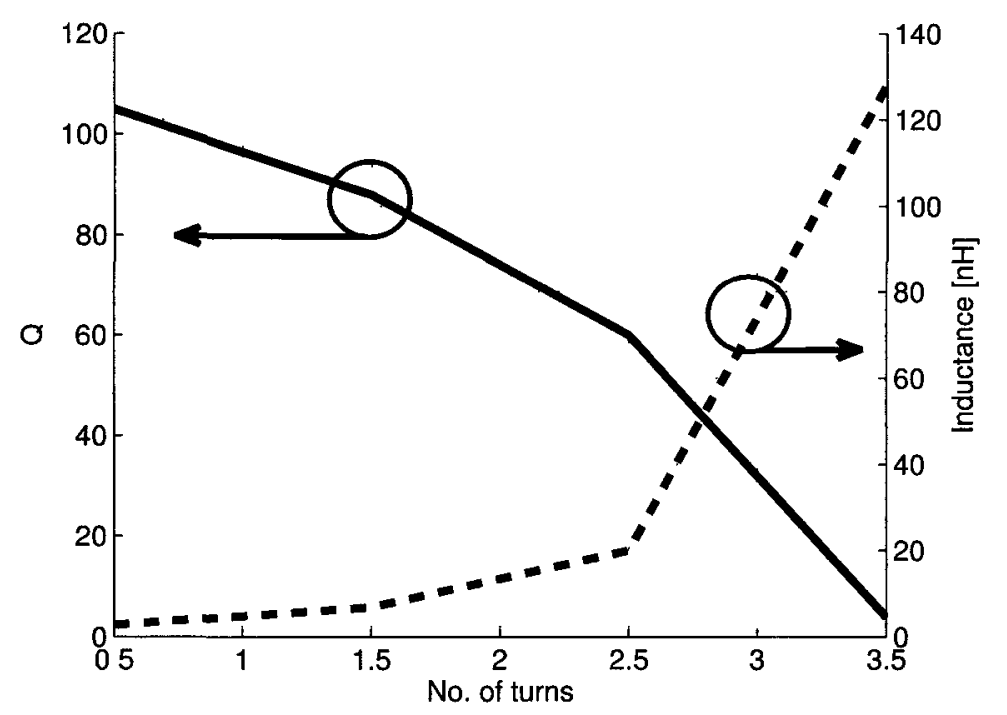

Figure 3.7: The relationship between inductance (L) and Q-factor for a spiral inductor.

to keep the number of turns low to achieve a high $\mathrm{Q}$ but the lower bound is determined by the minimum required inductance. In this work, 1.5 turns provided an acceptable amount of inductance for the highest Q. The Self-Resonant Frequency (SRF) should also be at least twice the center frequency to ensure a stable value of inductance. In this case, the SRF is more than 3 times higher. Importantly, selecting an odd multiple of 0.5 turns allows for an in-line geometry that simplifies the creation of a resonator. The input signal to the inductor is applied to G-S-G pads that have a pitch of $500 \mu \mathrm{m}$. The other end of the inductor is connected to an underlying ground with a via that has a diameter of $150 \mu \mathrm{m}$. Fig. 3.8 shows two different views of the inductor structure. 


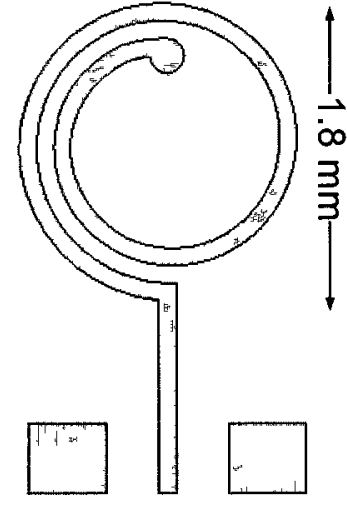

(a)

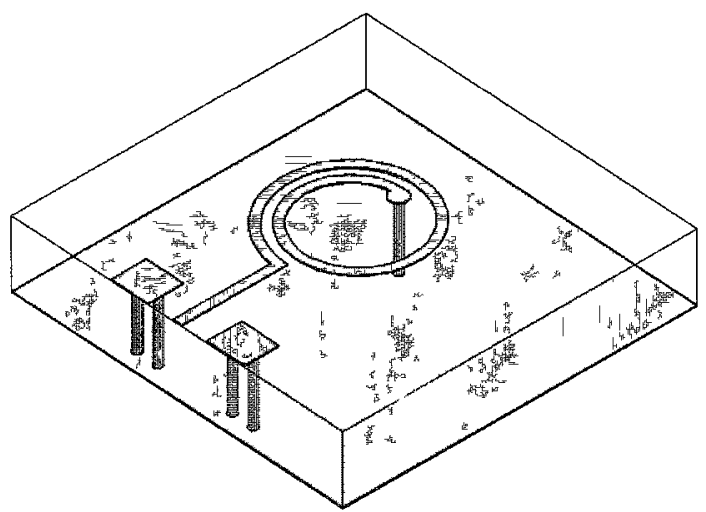

(b)

Figure 3.8: Spiral inductor model view from: (a) top and; (b) oblique. As indicated, the diameter is $1.8 \mathrm{~mm}$.

The values of inductance and Q-factor were calculated with the use of (3.1) and (3.2), respectively. They are valid for a one-port simulation.

$$
\begin{aligned}
& L=\frac{\operatorname{Im}\left(Z_{\imath n}\right)}{\omega} \\
& Q=\frac{\operatorname{Im}\left(Z_{\imath n}\right)}{\operatorname{Re}\left(Z_{\imath n}\right)}
\end{aligned}
$$

As shown in Section 3.6, the simulated inductance and Q-factor are $7.1 \mathrm{nH}$ and 86, respectively.

\subsubsection{Circular Multilayer Capacitor Design}

A multilayer capacitor with a plate separation of $100 \mu \mathrm{m}$ is employed to realize the component values for $C 3$ and $C 4$. The size of the plates is restricted to a maximum radius of $600 \mu \mathrm{m}$ to be compatible with the previously designed inductor. Given the limited area, a study was conducted to determine the optimal number of plates. Fig. 
3.9 shows the results of a parametric simulation for 1,2 , and 3 layer capacitors at $1.55 \mathrm{GHz}$.

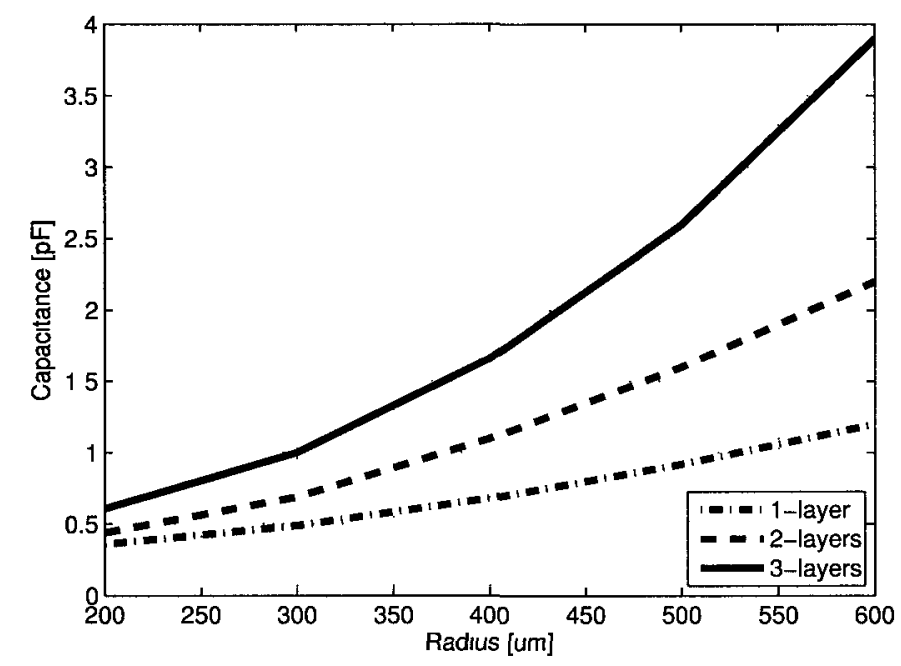

Figure 3.9: Gains in capacitance with the use of multiple layers.

As expected, for a given radius the two-layer structure has about twice the capacitance of the single layer version. Similarly, the three-layer structure has about triple the capacitance. However, its curve is not as linear as the others due to increased influence of fringing capacitances. In all cases the SRFs are well above the center frequency. Therefore, the three-layer structure can be safely used to realize $C 3$ and $C 4$ with significantly reduced area. For example, to obtain $1.5 \mathrm{pF}$, radii of $380 \mu \mathrm{m}$ and $490 \mu \mathrm{m}$ would be needed for a three-layer and two-layer structure, respectively (a reduction of $25 \%$ ).

The three-dimensional structure is shown in Fig. 3.10 along with a simplified equivalent circuit [6]. From the equivalent circuit it is apparent that the threelayer structure consists of three identical capacitors in parallel, $C P 1, C P \mathcal{2}$, and $C P 3$. Where, $R P$ represents the parallel combination of the resistive losses. The capacitance 


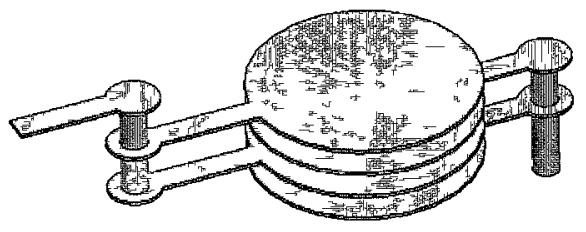

(a)

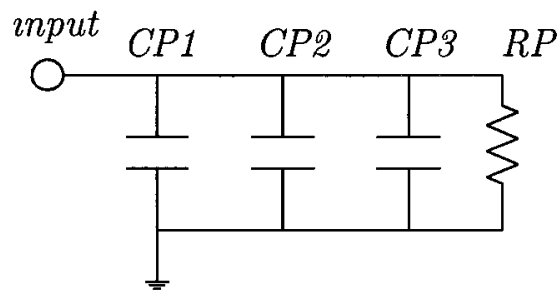

(b)

Figure 3.10: Multilayer capacitor: (a) model with identical radii for each plate and; (b) simplified equivalent circuit.

can be calculated from (3.3).

$$
C=\frac{\operatorname{Im}\left(Y_{11}\right)}{\omega}
$$

The required capacitances for $C 3$ and $C 4$ were realized by setting the radii of the plates to $335 \mu \mathrm{m}$ and $380 \mu \mathrm{m}$, respectively.

\subsubsection{Novel L-C Resonator Design}

The $\mathrm{L}-\mathrm{C}$ resonator is created by combining the previously designed inductor and capacitors; consequently, its diameter is the same as that of the inductor $(1.8 \mathrm{~mm})$. The capacitor is placed at the center of the inductor and two layers below its plane. Fig. 3.11 shows a top and oblique view of the resonator with the embedded capacitor visible. In this way, de-tuning and Q-degrading electromagnetic coupling is reduced. Fig. 3.12 displays the effect on $\mathrm{Q}$ of the capacitor placement within the structure. The unloaded Q-factor of the resonator is calculated with the use of (3.4) [23]:

$$
Q_{\text {res }}=\frac{R}{\omega_{0} L}
$$




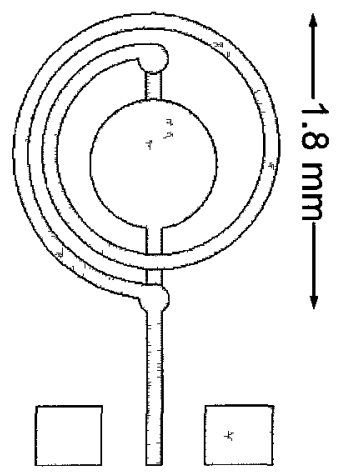

(a)

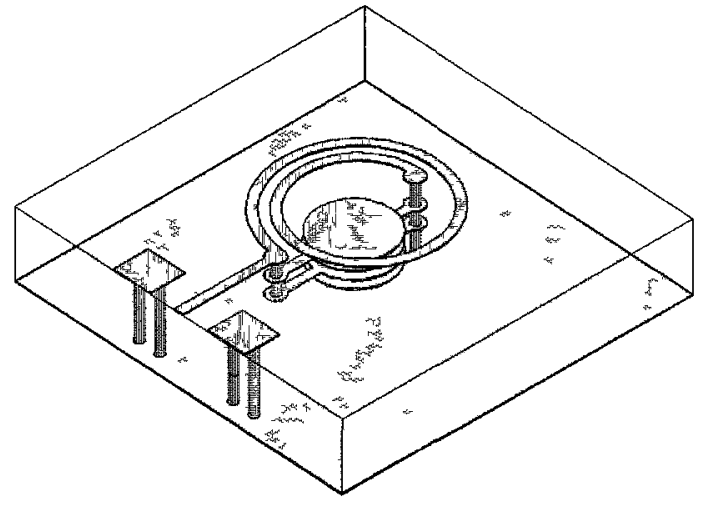

(b)

Figure 3.11 L-C resonator model view from (a) top and (b) oblique. As indicated, the diameter is $1.8 \mathrm{~mm}$.

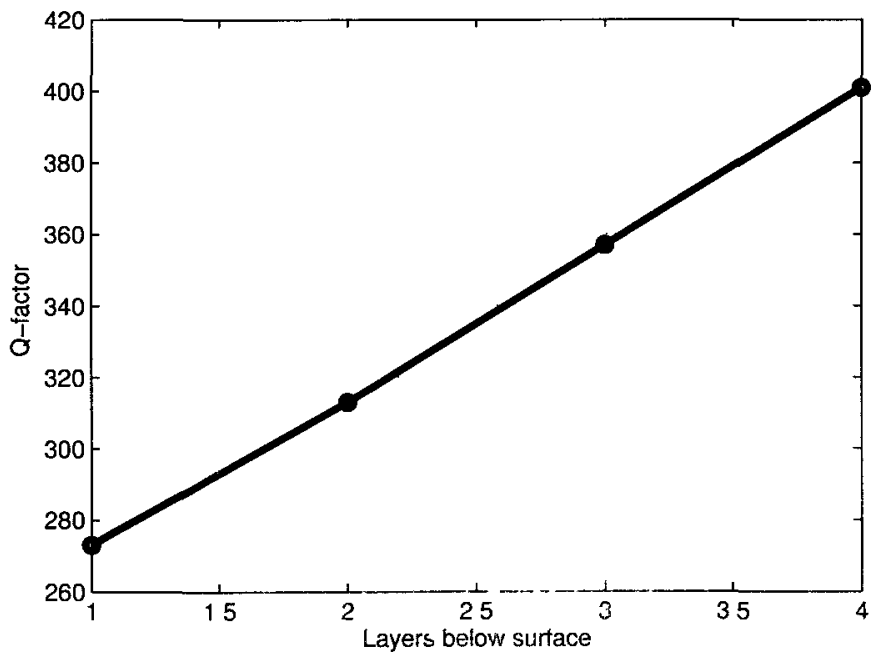

Figure 312 The effect on resonator $Q$ when increasing the separation between the parallel inductor and capacitor. 
where, $R$ is the input impedance at resonance, $\omega_{0}$ is the resonant frequency, and $L$ is the inductance of the parallel inductor. Complete characterization of the resonator performance is given in Section 3.6.

\subsubsection{Coupling Capacitor Design}

The design of the coupling capacitors proceeds in the same manner as those for the circular capacitors. The results of a parametric simulation yielded the dimensions of a two-layer, square plate capacitor that could implement C2. A length of $100 \mu \mathrm{m}$ yielded a capacitance close to the required $0.1 \mathrm{pF}$. For $C 1$, a four layer, square plate capacitor was used to save area. A length of $300 \mu \mathrm{m}$ yielded the necessary capacitance of $0.5 \mathrm{pF}$.

\subsection{LTCC Bandpass Filter Design}

With the completion of the component library, the next stage in the design process can begin, namely, the assembly of a hybrid EM model.

\subsubsection{Hybrid Filter Structure - Four Ideal Coupling Capaci- tors}

Here, all four of the coupling capacitors have been implemented with ideal impedance boundaries, while real resonators have been directly inserted. In accordance with the filter schematic of Fig. $3.2, C 1$ and $C 2$ were set to $0.1 \mathrm{pF}$ and $0.5 \mathrm{pF}$, respectively. Of course, ideal boundaries could have been used to represent the resonators also.

The assembled filter with four ideal coupling capacitors is shown in Fig. 3.3. 
The resonators are placed on alternating sides of the filter backbone. To reduce the magnetic coupling between the adjacent resonators, one resonator is mirrored such that the surrounding magnetic fields of each inductor oppose each other. The filter is fed by the same G-S-G pad structure as were the other components. The overall size of this filter is $5 \times 5.4 \times 0.8 \mathrm{~mm}^{3}$.

As expected, the initial simulated response did not meet the specifications. But because this is a hybrid structure with ideal coupling capacitors, only $C 3$ and $C 4$ needed to be tuned. After performing a short parametric simulation, a satisfactory filter response was obtained when the radii of the plates of $C 3$ and $C 4$ were set to $345 \mu \mathrm{m}$ and $430 \mu \mathrm{m}$, respectively. This shows that by using a hybrid EM structure, the initial simulation results can be easily improved to meet the specifications.

\subsubsection{Hybrid Filter Structure - Two Ideal Coupling Capaci- tors}

The next step involves replacing the inner $0.1 \mathrm{pF}$ ideal impedance boundaries with real coupling capacitors from the component library developed earlier. This version of the filter is shown in Fig. 3.5. Again, the initial filter response was less than ideal. However, it was clear that the major variable that needed to be tuned was the size of the newly inserted inner coupling capacitors. By enlarging their length to $256 \mu \mathrm{m}$ the filter response was significantly improved. Further improvement was obtained by slightly modifying the sizes of $C 3$ and $C 4$ so that their radii now measured $341.33 \mu \mathrm{m}$ and $438.3 \mu \mathrm{m}$, respectively. The overall size of this filter is $5 \times 5.4 \times 0.8 \mathrm{~mm}^{3}$. 


\subsubsection{Fully Embedded Filter Structure}

The final iteration in the design process involves replacing the remaining two ideal impedance boundaries with real $0.5 \mathrm{pF}$ coupling capacitors. With this final substitution a fully embedded filter is created. The initial simulation revealed a larger than expected insertion loss and reduced center frequency. By using a circuit simulator the exact causes of the performance degradation were determined. The insertion loss was improved by resizing capacitors $C 1$ and $C 2$ to $391 \mu \mathrm{m}$ and $450 \mu \mathrm{m}$, respectively. The center frequency was reduced because of significant capacitive coupling between the outer resonators and capacitors $C 1$ and $C 3$. This was expected because of their close proximity. This effect was countered by reducing the radii of $C 3$ to $270 \mu \mathrm{m}$ and $C_{4}$ to $420 \mu \mathrm{m}$, respectively. The overall size of this filter is $5 \times 6 \times 0.8 \mathrm{~mm}^{3}$.

Table 3.3 summarizes the differences between the initial library component dimensions and those from the final fully embedded filter.

Table 3.3: Comparison Between Initial and Optimized Component Dimensions

\begin{tabular}{|c|c|c|}
\hline Component & Initial Dimensions & Optimized Dimensions \\
\hline$C 1$ & $300 \mu \mathrm{m}$ & $391 \mu \mathrm{m}$ \\
\hline$C 2$ & $100 \mu \mathrm{m}$ & $450 \mu \mathrm{m}$ \\
\hline$C 3$ & $335 \mu \mathrm{m}$ & $270 \mu \mathrm{m}$ \\
\hline$C 4$ & $380 \mu \mathrm{m}$ & $420 \mu \mathrm{m}$ \\
\hline
\end{tabular}

Whereas $C 1, C 3$, and $C 4$ only required minor tuning, the optimized dimensions of C2 are significantly different. This relatively large re-sizing is due to the fact that $C_{2}$ is only a single-layer capacitor, whereas the others are three. Therefore, its re-sizing corresponds to only a slight change in actual capacitance. 


\subsection{Passive Component and Bandpass Filter Char- acterization}

In this section measured results are shown alongside simulated results for comparison purposes. Vector Network Analyzer (VNA) measurements were performed with G-S-G probes having $500 \mu \mathrm{m}$ pitch and using a Short-Open-Load-Through (SOLT) calibration to the probe tips.

\subsubsection{Inductor Characterization}

As the single most critical component of the filters here, the circular spiral inductor was fully characterized. Fig. 3.13 shows the simulated and measured Q-factor for the inductor versus frequency [7]. It is evident that the measured Q-factor is noticeably

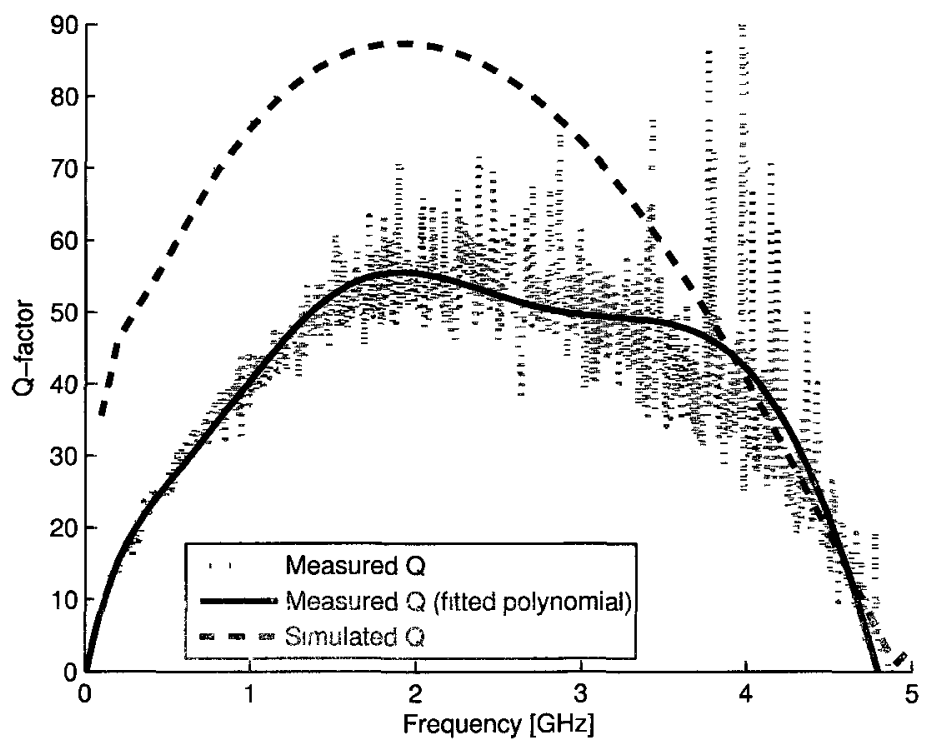

Figure 3.13: Measured and simulated Q-factor for inductor. 
lower than expected. This degradation can be explained by examining a micrograph of the inductor metallization shown in Fig. 3.14 [7]. The metallization appears to

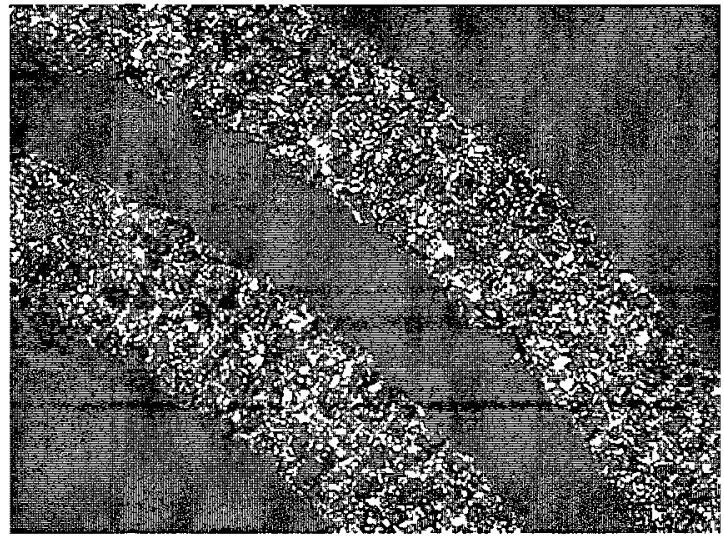

Figure 3.14: Inductor metallization micrograph.

have an inconsistent thickness with many areas showing no conductor present at all. Such a high degree of defects will increase the resistive losses of the trace and this is reflected in the lower than expected Q-factor of the inductor.

Fig. 3.15 shows the simulated and measured inductance versus frequency. The excellent agreement between these results validates the measurement procedure and confirms the explanation of the Q-factor results above [7]. From Fig. 3.15, the value of inductance is $7.1 \mathrm{nH}$ at the center frequency of $1.55 \mathrm{GHz}$ and the self-resonant frequency occurs near $5 \mathrm{GHz}$.

\subsubsection{Circular Multilayer Capacitor Characterization}

Several copies of $C_{3}$ and $C_{4}$ from each filter design were fabricated and characterized. Due to the similarity in the results obtained, only the results for capacitor $C 3$ from the first filter is demonstrated here. Fig. 3.16 shows a comparison of the measured 


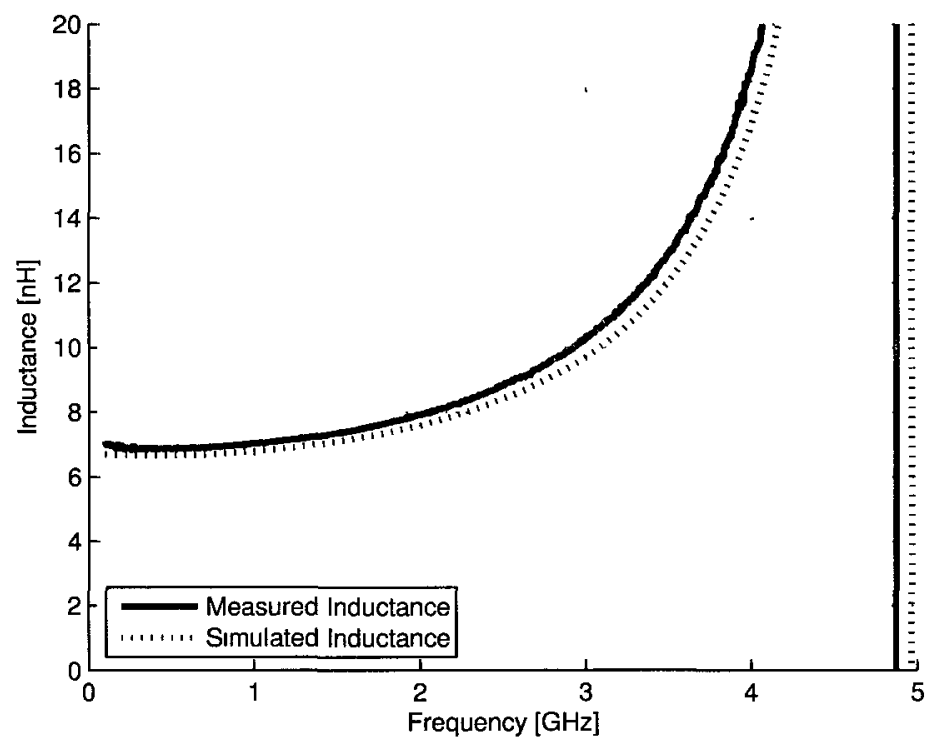

Figure 3.15: Measured and simulated inductance.

and simulated capacitance from $1 \mathrm{GHz}$ to $2 \mathrm{GHz}$. Similarly, Fig. 3.17 shows the Qfactor of this capacitor where the measured result appears noisy due to the fact that the real part of the input impedance is very small $(<1 \Omega)$. Therefore, a quadratic function is fitted to the data so that a meaningful comparison with the simulations may be done.

The results are in reasonably good agreement, with the measured capacitance being approximately $0.2 \mathrm{pF}$ lower than expected across the whole band. It is important to note that this trend was witnessed in all capacitor measurements for $C_{3}$ and $C_{4}$ and will be explained in Section 3.7. At the center frequency, the measured $\mathrm{Q}$ of the capacitor is approximately $9 \%$ lower than predicted by the simulation, 208 versus 245, respectively. An explanation for the differences between these measurements and their respective simulations is given in Section 3.7 . 


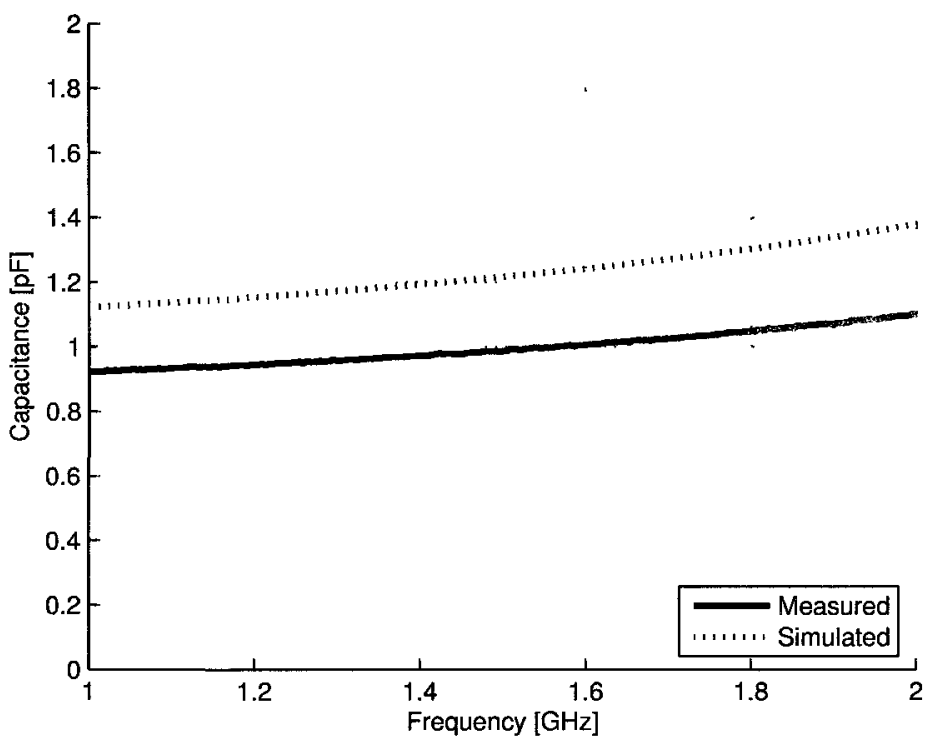

Figure 3.16: Measured and simulated capacitance of $C$ 3.

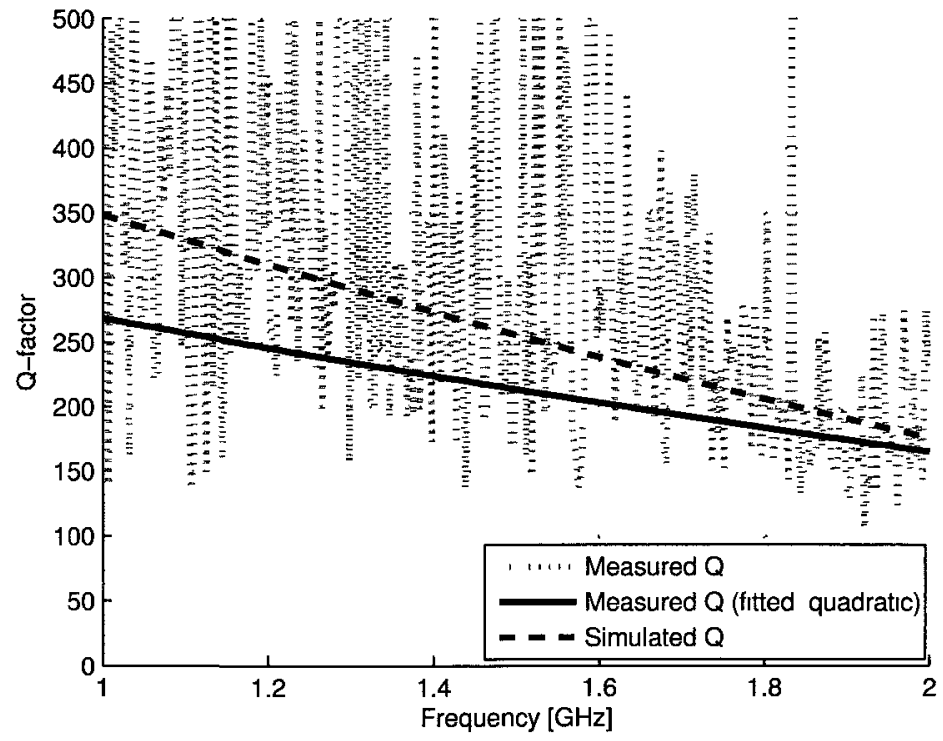

Figure 3.17: Measured and simulated Q-factor of $C 3$. 


\subsubsection{Novel L-C Resonator Characterization}

Each resonator from all three filters was fully characterized. However, for demonstration only the middle resonator of the fully embedded filter is shown here. The real and imaginary components of the resonator input impedance, Zin, are plotted separately in Fig. 3.18. Based on the peak of the real component of the input impedance, the
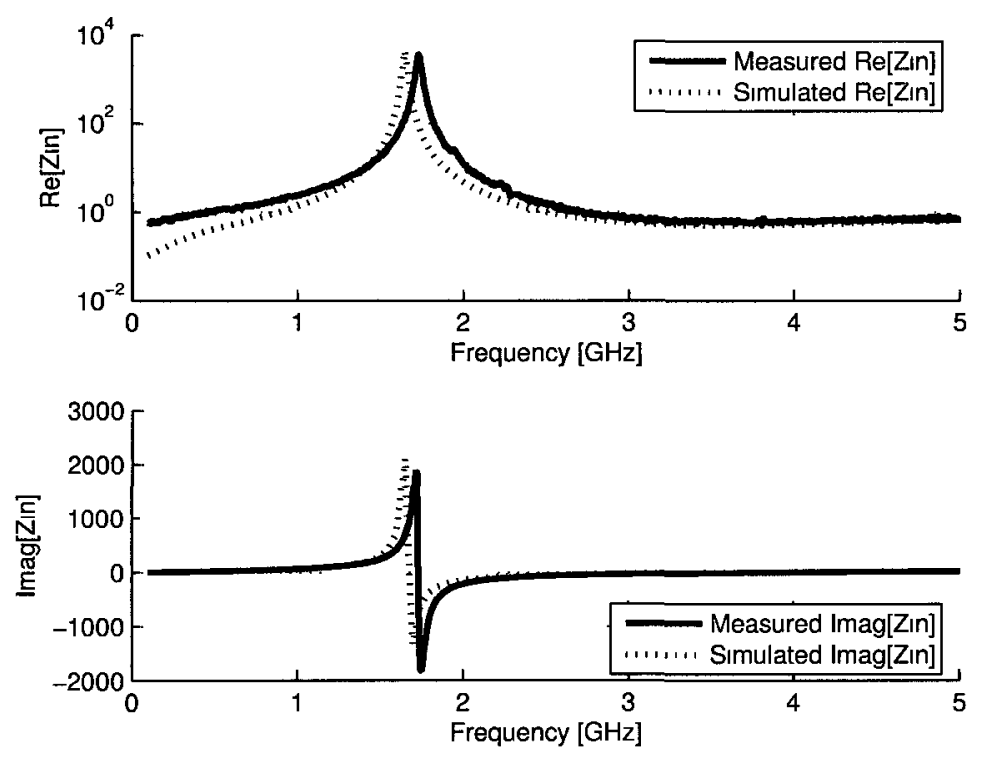

Figure 3.18: Measured and simulated resonator Zin.

resonant frequency occurs at $1.734 \mathrm{GHz}$. The unloaded Q-factor of the resonator can be calculated with the use of (3.4) by substituting the measured input impedance at resonance $(3621 \Omega)$ for $R$ and from Fig. 3.15 the measured inductance $(7.28 \mathrm{nH})$ for $L$. Performing the calculation yields a measured unloaded Q-factor of 45.65. This compares to the simulated Q-factor of 58 , where $R, w_{0}$ and $L$ are $4326 \Omega, 10.37 x 10^{6}$ rads and $7.19 \mathrm{nH}$, respectively. 


\subsubsection{Coupling Capacitor Characterization}

Individual coupling capacitors ( $C 1$ and C2) from the fully embedded filter were fully characterized. Fig. 3.19 shows a comparison of the measured and simulated capacitance from $1 \mathrm{GHz}$ to $2 \mathrm{GHz}$ for both $C 1$ and $C 2$.

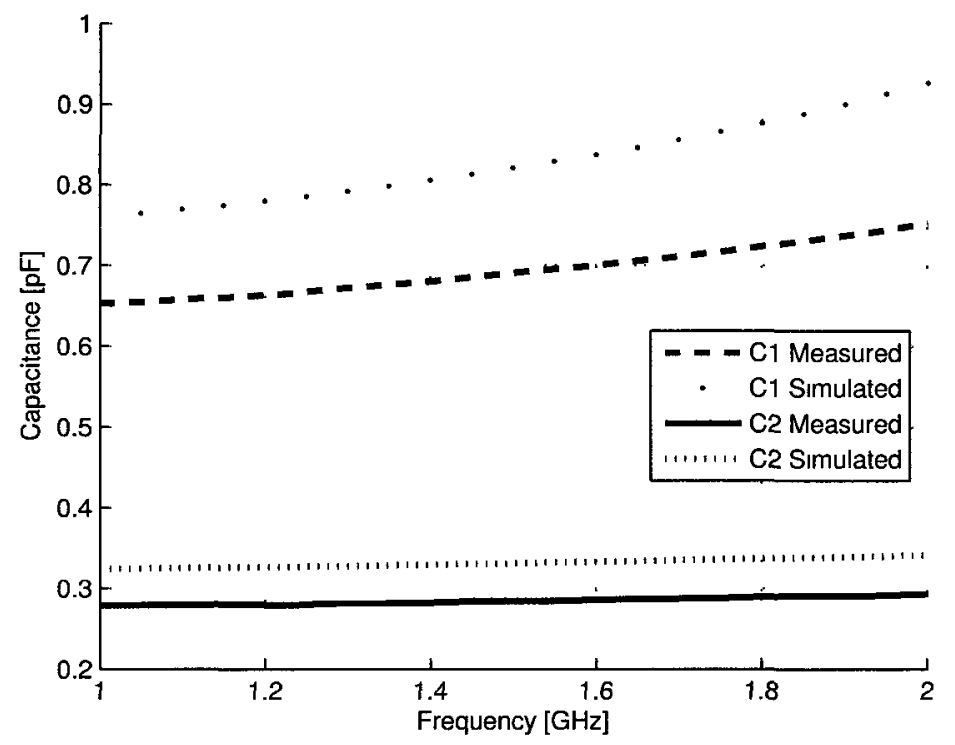

Figure 3.19: Measured and simulated coupling capacitors of fully embedded filter.

The same trend witnessed in the circular capacitor results continues here. That is, the simulated results overestimate the true value of capacitance. It is interesting to note that the error is greater for $C 1$, which has four layers as opposed to only two for C2. It is concluded that the error is not due to inaccurate simulations but rather to greater than expected manufactured layer thickness (since capacitance is inversely proportional to the layer thickness between capacitor plates). 


\subsubsection{Filter Characterization}

The measured and simulated filter responses are shown here for the filter with four ideal coupling capacitors (Fig. 3.20) and for the fully embedded filter (Fig. 3.24). For the former, the ideal capacitors are replaced with high Q ceramic capacitors with dimensions of $0.6 \times 0.3 \times 0.23 \mathrm{~mm}^{3}$. These were then mounted to the surface of the LTCC substrate with conductive epoxy. Fig. 3.21 shows a micrograph of two of these capacitors adjacent to the middle resonator.

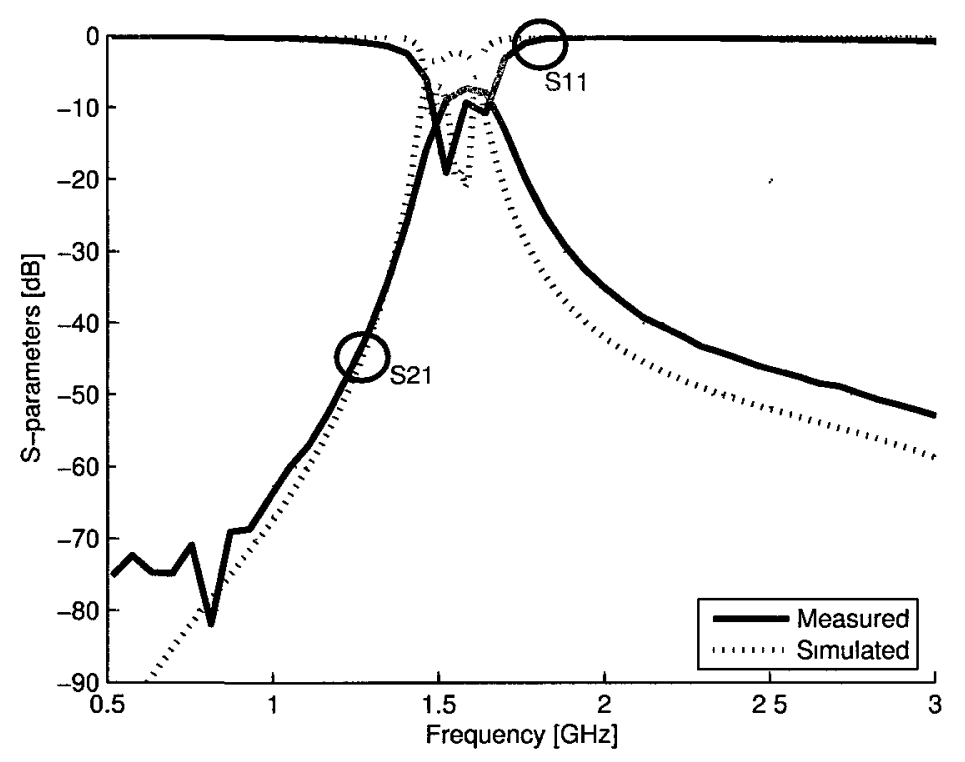

Figure 3.20: Measured and simulated response for filter with 4 surface mount capacitors.

For this filter, the measured return loss is below $10 \mathrm{~dB}$ for $\pm 50 \mathrm{MHz}$ around the center frequency of $1.6 \mathrm{GHz}$. The insertion loss is $7.25 \mathrm{~dB}$, while the stopband attenuation is greater than $30 \mathrm{~dB}$ beyond $\pm 300 \mathrm{MHz}$ of the center frequency. These results are in good agreement with the simulations except for the insertion loss in 


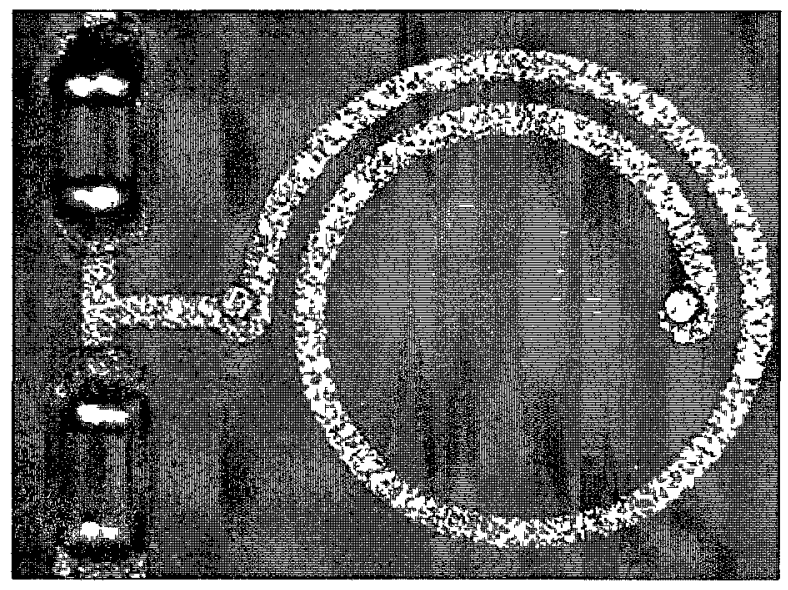

Figure 3.21: Micrograph of affixed surface mount coupling capacitors.

the passband. To understand the cause for this difference, multiple copies of this exact filter design were populated with surface mount capacitors and measured. The ceramic capacitors used to realize $C 1$ and C2 both have a published tolerance of $50 \%$ - as is typical for small valued ceramic capacitors. Large differences in performance were observed among these filters, which indicates a strong sensitivity of the filter response to capacitor variations. Fig. 3.22 shows the effect of $C 1$ tolerance on the insertion loss of the filter.

From Fig. 3.22, a capacitance for $C 1$ that is smaller than expected increases the insertion loss of the filter substantially. Clearly, it would be advantageous to avoid the use of small valued surface mount capacitors. To this end, the fully embedded filter is developed. A micrograph of the top metal layer is shown in Fig 3.23, while its performance is shown in Fig. 324.

The fully embedded filter displays a $10 \mathrm{~dB}$ bandwidth from $1.6 \mathrm{GHz}$ to $1.75 \mathrm{GHz}$. The return loss is $21.3 \mathrm{~dB}$ at the center frequency of $1.64 \mathrm{GHz}$. The cause of the 


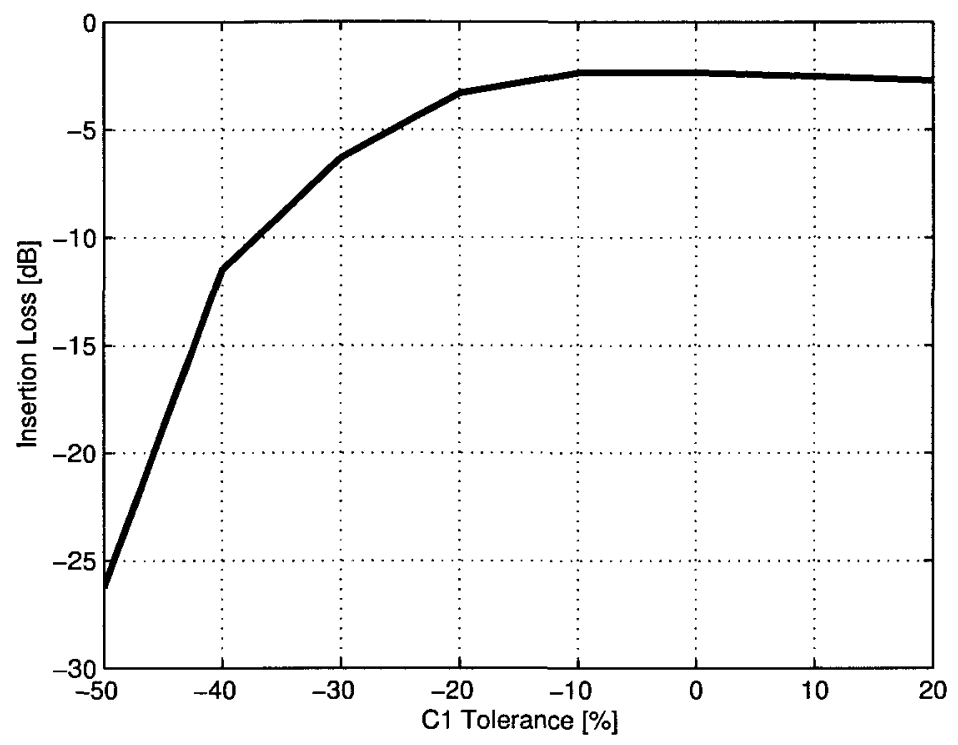

Figure 3.22: Simulated insertion loss variation due to $\mathrm{C} 1$ tolerance.

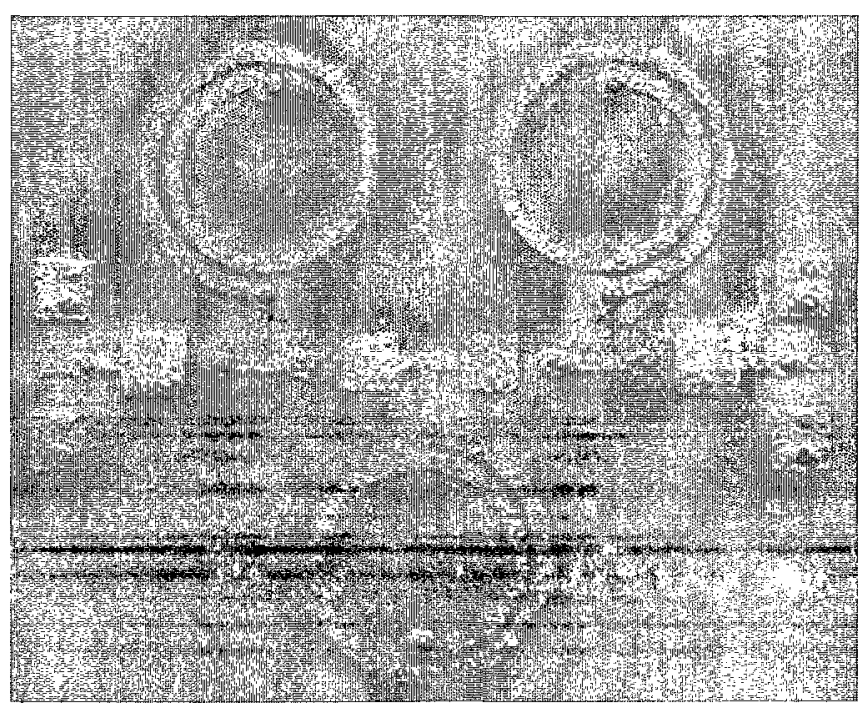

Figure 3.23: Micrograph of fabricated fully embedded bandpass filter (top view). 


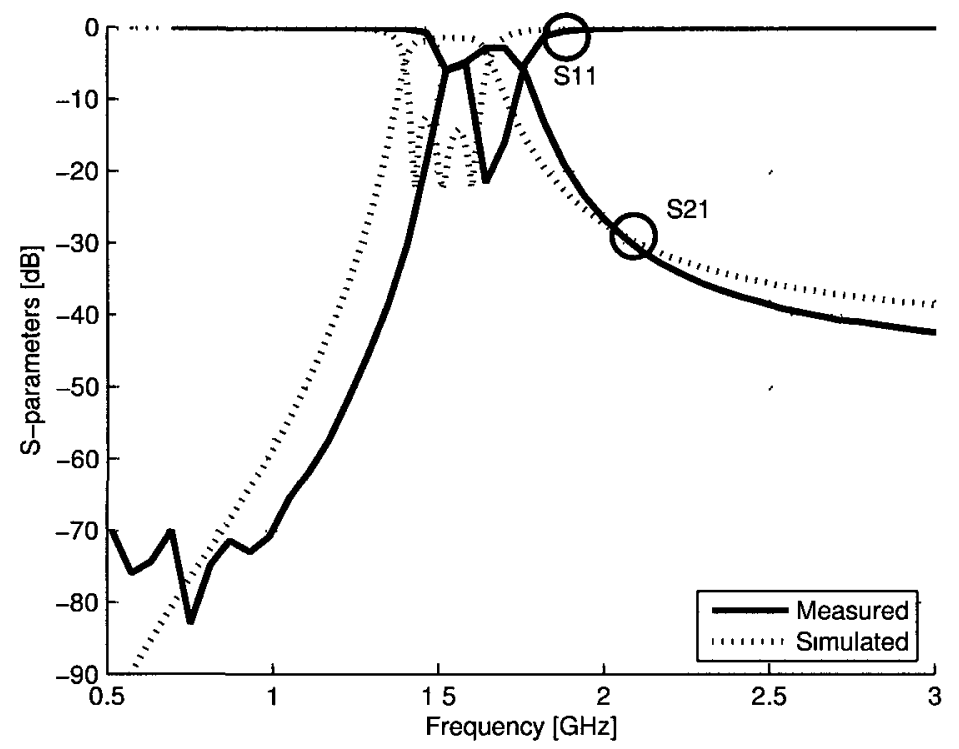

Figure 3.24: Measured and simulated response for fully embedded filter.

positive frequency shift of the passband is due to a combination of effects but primarily, a thicker than expected layer thickness and lower than expected substrate permittivity are at fault. Identical trends are reported in [77]. More detailed explanations of the thickness and permittivity effects are discussed in Sections 3.7.1 and 3.7.2, respectively. The insertion loss is greatly improved compared to the previous filter, being $2.8 \mathrm{~dB}$ and the stopband performance is similar. The insertion loss is improved because the very high tolerance surface mount capacitors of the previous filter are replaced with LTCC embedded capacitors, for which the $\mathrm{x}-\mathrm{y}$ size tolerance is typically $0.2 \%[78]$. 


\subsection{LTCC Process Tolerance Effects}

Comparing the filter results shows that surface mount component value tolerances have a large effect on filter performance. As shown by the fully embedded version, which lacks any surface mount components, LTCC process tolerances still have a noticeable influence on the filter response. The tolerances associated with layer thickness, dielectric constant, and metallization quality are analyzed in the following sections.

\subsubsection{Influence of Layer Thickness Tolerance}

The measured results show that the center frequency for each filter displays a positive shift of approximately $100 \mathrm{MHz}$. This effect can be explained by recalling that every measured capacitor displays a lower than expected capacitance. For example, in Section 3.6.2 the capacitance of $C 3$ is measured to be $20 \%$ lower than expected (or $6.67 \%$ lower per layer of the three layer capacitor). Thus, this reduced capacitance tends to shift the passband higher in frequency. The reduced capacitance is due to a substrate thickness that is measured to be $60 \mu \mathrm{m}$ thicker than requested. This represents a positive $7 \%$ variation in layer thickness and agrees very well with the $6.67 \%$ per layer reduction in capacitance observed (since capacitance is inversely proportional to layer thickness). This result falls at the extreme edge of the $\pm 7 \%$ tolerance range published by the LTCC foundry [78].

\subsubsection{Influence of Dielectric Constant}

The analysis of a calibration structure on the same substrate as the filters indicates that the substrate permittivity is $5 \%$ lower than expected. This variation falls within 
the manufacturers published tolerance but nonetheless also has the effect of increasing the center frequencies (since capacitance is directly proportional to substrate permittivity).

\subsubsection{Influence of Metallization Quality}

From the micrographs, the surface roughness of the top metal layer was shown to be much greater than expected. Since this layer contains the spiral inductors, their Qfactors were substantially reduced by the extra resistive losses imposed. The degraded Q-factors of the inductors and resonators, of which they are part, manifests itself in the measurements as higher insertion losses for both filters.

\subsubsection{Post Simulated Results}

Having quantified the errors due to layer thickness and dielectric constant, it is now possible to perform a post-simulation comparison. It is expected that by increasing the thickness of each layer by $7 \%$ (or $7.5 \mu$ ) and reducing the dielectric constant by $5 \%$ to 5.605 will increase the agreement between the simulations and measurements. The results of these changes are shown in Fig. 3.25 under the label of "post-simulated." The changes have the desired effect of improving the agreement with the measurements. Therefore, it may be concluded that the tolerance effects quantified above are accurate. Based on this experience, extra efforts were made to obtain more accurate material specifications on which the designs of later chapters are based.

\subsubsection{Performance Summary}

In Table 3.4, the performance of this filter is summarized. The performance is rea- 

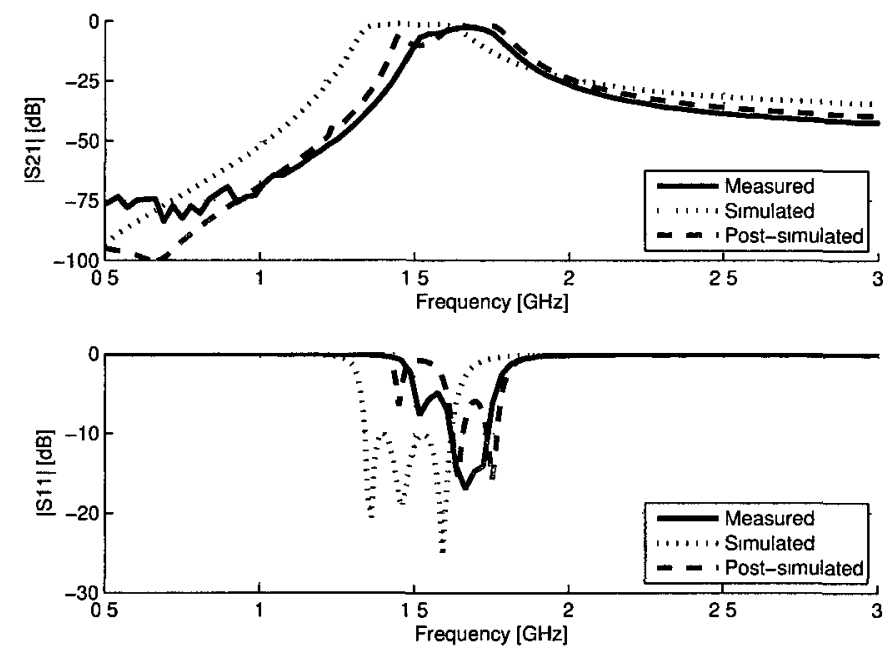

Figure 3.25: A comparison of the measured, simulated, and post-simulated results for fully embedded filter.

Table 3.4: Performance Summary

\begin{tabular}{|c|c|c|c|}
\hline Item & Frequency & Simulated Value & Measured Value \\
\hline Center Frequency & {$[1550 \mathrm{MHz}]$} & {$[-]$} & $1640 \mathrm{MHz}$ \\
\hline $10 \mathrm{dR}$ Return Loss & {$[$ in-band] } & $1340-1620 \mathrm{MHz}$ & $1620-1730 \mathrm{MHz}$ \\
\hline Insertion Loss & {$[$ in-band] } & $1.5 \mathrm{~dB}$ & $2.8 \mathrm{~dB}$ \\
Attenuation & {$[2000$ to $6000 \mathrm{MHz}]$} & $24 \mathrm{~dB}(\min )$ & $27 \mathrm{~dB}(\min )$ \\
& {$[1442 \mathrm{MHz}]$} & $1.5 \mathrm{~dB}$ & $20 \mathrm{~dB}$ \\
& {$[1660 \mathrm{MHz}]$} & $6 \mathrm{~dB}$ & $3 \mathrm{~dB}$ \\
\hline
\end{tabular}


sonably good despite the frequency shift caused by material tolerances. This result will serve as a basis for new and improved designs presented in the next chapters.

\subsection{Summary and Contribution}

This chapter has presented a new, simpler design methodology that has been demonstrated to be effective through the realization of highly miniaturized LTCC bandpass filters. The entire process, from initial schematic design, through individual element design and characterization, to complete filter characterization has been discussed. The new bandpass filters have desirable performance characteristics for emerging GPS applications. Although reasonable agreement was obtained, all sources of error related to LTCC process tolerance effects were analyzed. The specific publications arising from the work contained in this chapter includes two conference papers and one Journal article [5-7].

The results of this chapter constitute the first and second thesis contributions, namely the demonstration of a novel embedded L-C resonator and the application of a novel design methodology to a miniaturized LTCC bandpass filter design. Important lessons and insights have been learned though the process and will be applied to the design of improved versions with even higher performance and smaller footprint. 


\section{Chapter 4}

\section{Advanced BPF Designs}

This chapter continues to pursue the third thesis objective through the design of improved filters. More complex topologies and a higher degree of miniaturization are used to obtain better performance and packaging. As presented in Fig. 4.1, the filter's purpose is to attenuate out of band signals.

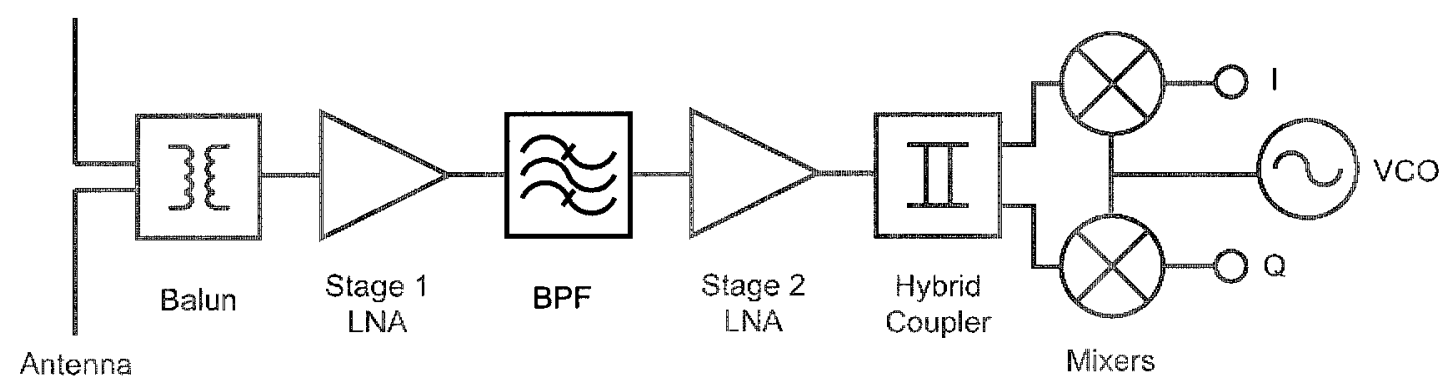

Figure 4.1: Bandpass filter as part of the complete receiver chain.

With ever more stringent filter requirements, conventional filter topologies are becoming inadequate. The filters of Chapter 3 could be augmented by increasing their order. This would provide steeper roll-off at the passband edges and increased outof-band rejection. However, increased in-band insertion loss would be an unavoidable consequence, along with larger size. Here, two advanced filter techniques are utilized 
to make the improvements stated above possible without increasing insertion loss or size.

These novel filters achieve superior performance while occupying significantly less area. A second-order filter is presented that displays an insertion loss of $2.2 \mathrm{~dB}$. For better out-of-band performance, a fourth-order filter is presented that displays steep rejection skirts and an insertion loss of less than $4 \mathrm{~dB}$. Both of these filters exhibit roll-offs that are higher than their conventional equivalents due to additional zeros placed at the passband edges.

\subsection{Finite Transmission Zeros}

Conventional filter topologies that are composed of coupled parallel resonators do not have transmission zeros (except at $f=0$ and $f=\infty$ ) [79]. However, modifications to the coupling mechanisms can introduce transmission zeros in the stopband, thereby enhancing the rejection characteristics. Many different combinations of reactive coupling can be used. Capacitive cross coupling of the input and output will generate a zero at frequencies below the passband [80]. An arbitrary number of zeros can be generated with parallel resonators in series or shunted series resonators [81]. Here, capacitive feedback from input to output and inductive coupling between resonators is implemented because the zeros can be located accurately $[82,83]$.

To demonstrate the advantages of these methods, a second-order filter is designed. Such a topology will also provide added benefit of minimizing the passband insertion loss since, in general, insertion loss decreases with lower order filters [23]. 


\subsubsection{Capacitive Coupling}

Fig. 4.2 shows a conventional second-order coupled resonator filter alongside its typical response.

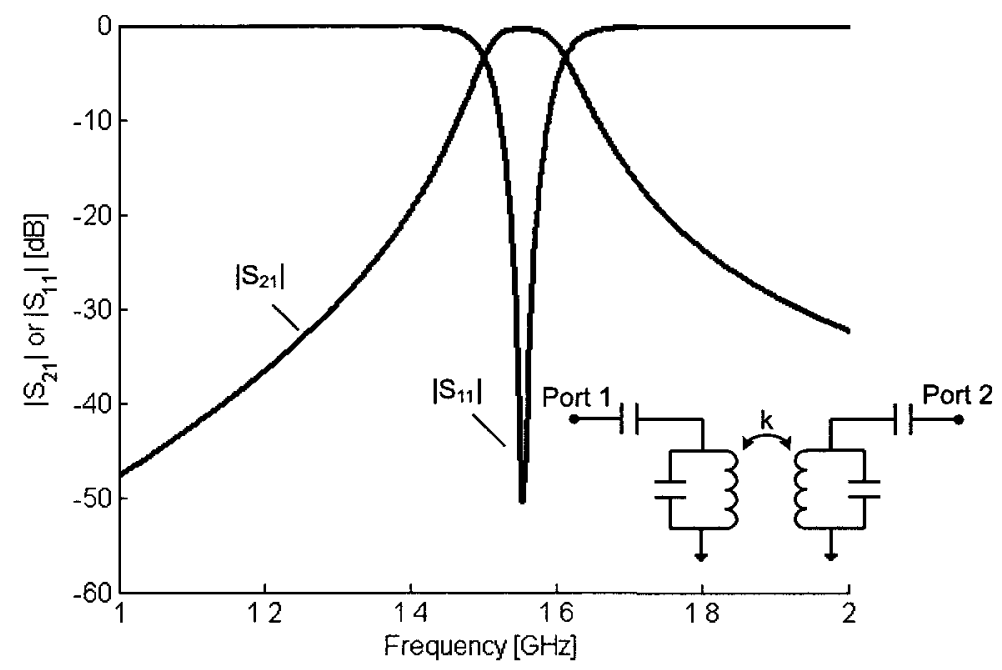

Figure 4.2: Second-order filter with no feedback (schematic and typical response).

The slope at the passband edges is approximately $60 \mathrm{~dB}$ per octave and the stopband rejection is about $40 \mathrm{~dB}$. In comparison, Fig. 4.3 shows a second-order filter schematic with capacitive feedback that creates two new finite transmission zeros. The associated response shows a a roll-off slope of approximately $120 \mathrm{~dB}$ per octave (with linear extrapolation), which is double that of the first case. However, the tradeoff is a noticeably lower stopband rejection of about $20 \mathrm{~dB}$.

This example has shown that with the introduction and proper sizing of a feedback capacitor two finite transmission zeros can be placed very near the passband edges. This creates a sharp roll-off normally associated with higher-order filters. Furthermore, the insertion loss in the passband is largely unaffected compared to the 


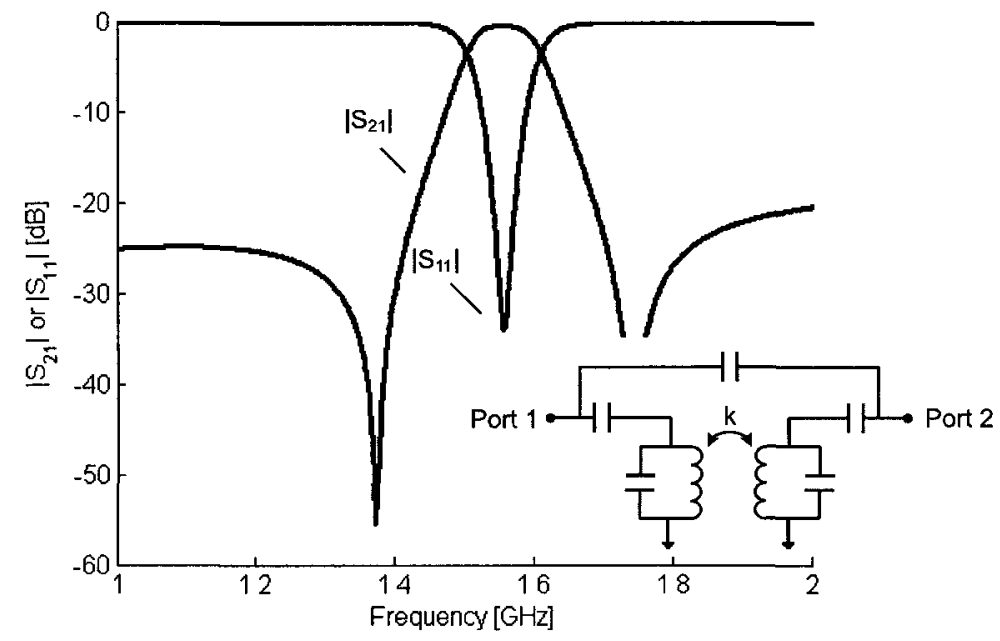

Figure 4.3: Second-order filter with capacitive feedback showing two finite transmission zeros (schematic and typical response).

conventional filter. However, the trade-off is lower stopband rejection. A filter design based on this topology has been fabricated and fully characterized. The results are presented in the following sections.

Additionally, a fourth-order filter is also designed to demonstrate increased selectivity. This filter is based on a capacitively coupled resonator topology with inductive coupling. The circuit schematic along with its corresponding response (with and without inductive coupling) is presented in Fig. 4.4.

In the first example, it is capacitive coupling that creates a feedback path and resultant transmission zeros. Here, inductive coupling serves the same purpose. The location of the zeros can be controlled by the degree of coupling. Stronger coupling brings the zeros closer to the passband edges. At $1.4 \mathrm{GHz}$ and $1.86 \mathrm{GHz}$ (locations of zeros), the rejection is more than $30 \mathrm{~dB}$ greater compared to the case without coupling. The trade-off is reduced in-band return loss. A filter based on this topology has also 


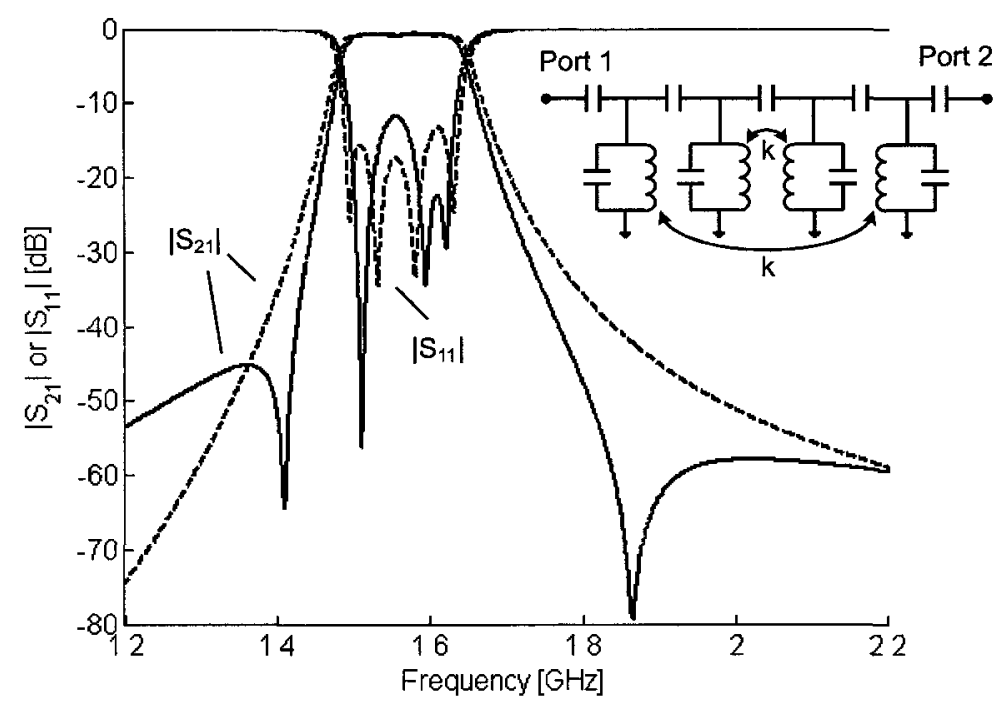

Figure 4.4: Fourth-order filter with inductive coupling (solid line) and without (dotted line) (schematic and typical response).

been designed and fully characterized with the results contained in the following sections.

These two examples have shown that inductive or capacitive coupling schemes can effectively increase the order of a given filter without any extra resonators - at least in terms of the roll-off at the passband edges.

\subsection{Challenges}

Two major challenges need to be overcome to create a miniature filter with the features discussed earlier: i) sizing of feedback capacitor to accurately place transmission zeros and ii) spacing of inductors to obtain the correct amount of inductive coupling.

Transmission zeros can be placed at an arbitrary frequency based on the value of the feedback capacitor. For example, the zeros can be placed at a frequency that 
coincides with a known unwanted Voltage Controlled Oscillator (VCO) harmonic to reduce its power. Alternatively, the zeros can be placed near the passband to increase filter selectivity - this approach is used here. The first challenge herein is how closely the zeros can be placed to the passband without worsening the insertion loss. Using inductively coupled resonators (instead of capacitively coupled) effectively reduces the filters' component count. However, the trade-off is increased complexity associated with the inductors. The second challenge herein is to design a shape and spacing that achieves the desired degree of coupling.

\subsection{Improved Second-Order Filter Design}

The same design procedure was followed as in Chapter 3, while the specifications are also unchanged but repeated in Table 4.1 for convenience. Given the circuit schematic

Table 4.1: Filter Specifications

\begin{tabular}{|c|c|}
\hline Center Frequency $[\mathrm{GHz}]$ & 1.55 \\
\hline $10 \mathrm{~dB}$ Bandwidth $[\mathrm{MHz}]$ & 100 \\
\hline Insertion Loss $[\mathrm{dB}]$ & $<2.5$ \\
\hline Stopband Attenuation $[\mathrm{dB}]$ & $>20$ at $\pm 150 \mathrm{MHz}$ offset \\
\hline
\end{tabular}

in Fig. 4.5, all component values were optimized to meet the stated specifications. Using the component values listed in Table 4.2, the response presented in Fig. 4.3 is obtained.

The value of the feedback capacitor, $C 2$, was chosen to provide transmission zeros as near to the passband edges as possible without affecting the insertion loss or the stopband attenuation too adversely. All these component values can be reasonably implemented in standard low permittivity LTCC material. To realize the small 


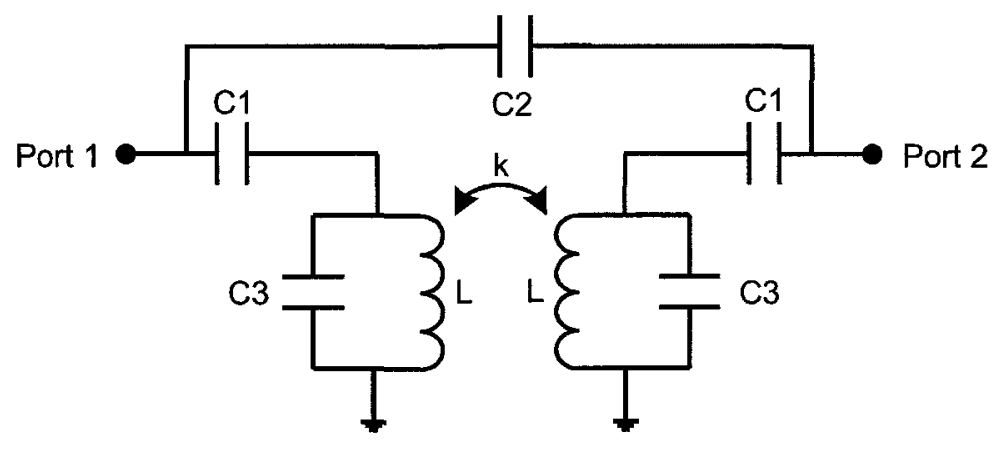

Figure 4.5: Improved Second-order filter with inductive and capacitive coupling.

Table 4.2: Theoretical Component Values For Improved Second-Order Bandpass Filter

\begin{tabular}{|c|c|}
\hline Component & Value \\
\hline$L$ & Ind. $=3.55 \mathrm{nH}$ \\
\hline$C 1$ & $0.57 \mathrm{pF}$ \\
\hline$C 2$ & $0.1 \mathrm{pF}$ \\
\hline$C 3$ & $2.42 \mathrm{pF}$ \\
\hline$k$ & $5 \%$ or $0.1775 \mathrm{nH}$ \\
\hline
\end{tabular}


coupling factor $(k=0.1775 \mathrm{nH})$, a pair of identical inductors that reside on the same metal layer and have magnetic moments that appose each other were designed. If the moments were made to align, too much coupling would be created. In Fig. 4.6(a), the HFSS model with a pair of coupled planar inductors is shown. The spiral that

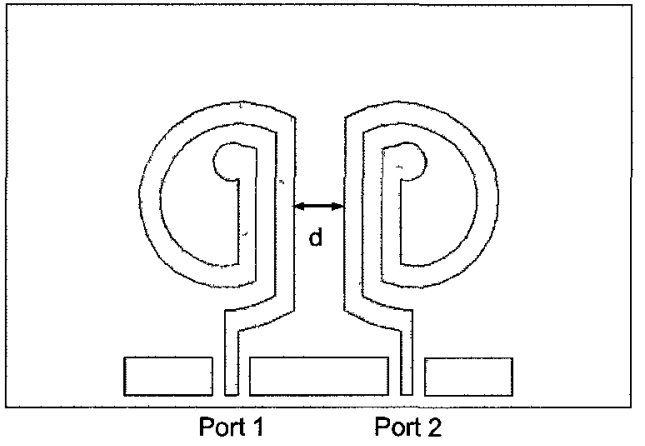

(a)

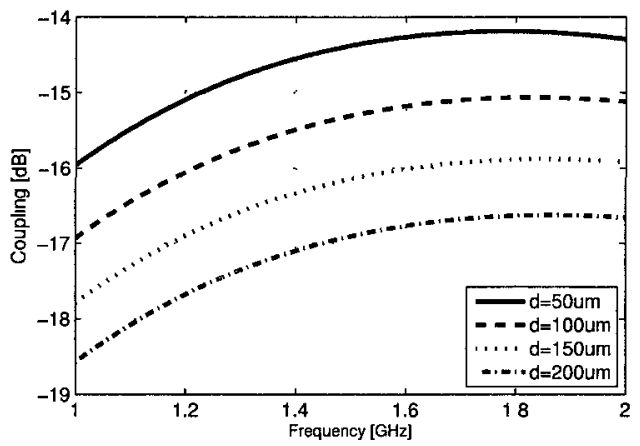

(b)

Figure 4.6: Simulated inductor coupling: (a) model; and (b) plot of coupling strength for various separation distances.

forms the inductor is truncated on one side to make a straight edge allowing for a much smaller pitch (center-to-center distance) between pairs of inductors and thus better controlled coupling. The desired degree of coupling can be obtained by adjusting the separation as shown in Fig. 4.6(b). As expected, the coupling is inversely proportional to the separation distance. The theoretical coupling required is $5 \%$ or $-13 \mathrm{~dB}$ and from Fig. 4.6(b), this corresponds to approximately a $50 \mu \mathrm{m}$ separation. This inductor pair is the structure around which the three-dimensional filter layout will be completed. 


\subsubsection{Resonator Configuration}

The main goal in the layout of the filter is area minimization. To achieve the smallest layout, as many components as possible should be stacked. This of course will cause unwanted mutual coupling so some optimization will be needed. As before, a stacked resonator configuration is utilized with the coupled inductors placed on the top metal layer and the multi-plate resonator capacitors $(C 3)$ below. The feedback capacitor $(C 2)$ and the input capacitors $(C 1)$ are also placed below the inductors and above the $C 3$ capacitors. In this way, only seven layers of LTCC are needed to package the complete filter. Images of the three-dimensional model are presented in Fig. 4.7.

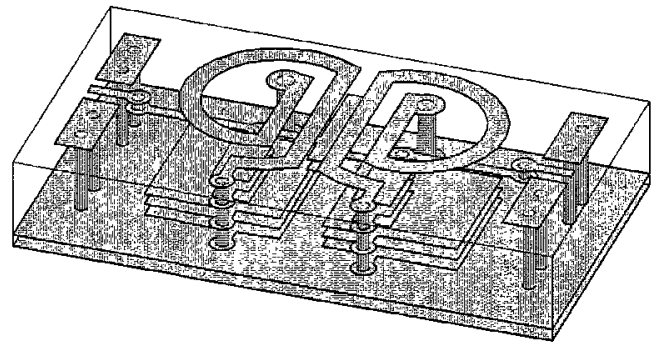

(a)

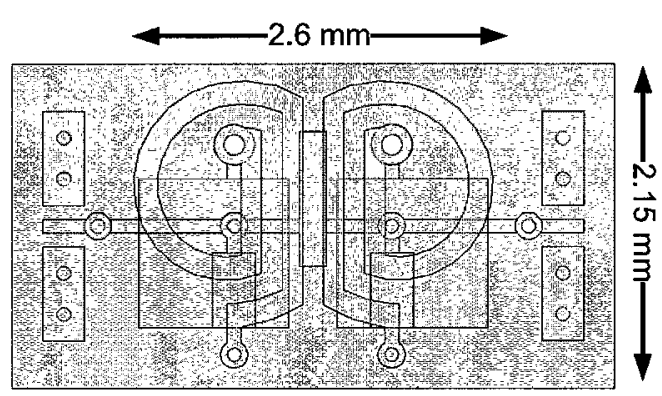

(b)

Figure 4.7: Three dimensional layout of new second-order filter: (a) oblique view; and (b) top view.

The embedded capacitors can be seen more clearly in Fig. 4.7(b) where the layers are made transparent. The elongated rectangular feedback capacitor is located at the center of the filter and is printed on layers 6 and 7. Meanwhile, the input capacitors are placed on layers 5 and 6 . The larger resonator capacitors occupy layers 2,3 , and 4 . The filter core, which excludes the probe pads, measures only $2.6 \times 2.15 \times 0.679 \mathrm{~mm}^{3}$ 
or $0.032 \lambda_{g} \times 0.026 \lambda_{g} \times 0.008 \lambda_{g}$, which makes this the smallest reported L-band second-order bandpass filter. The following sections provide simulated and measured results for the filter as a whole and its individual passive components.

\subsubsection{Improved Fourth-Order Filter Design}

A fourth-order filter may be constructed by connecting in series two second-order filters. Such a topology is shown in Fig. 4.8. Inductive coupling is used between the

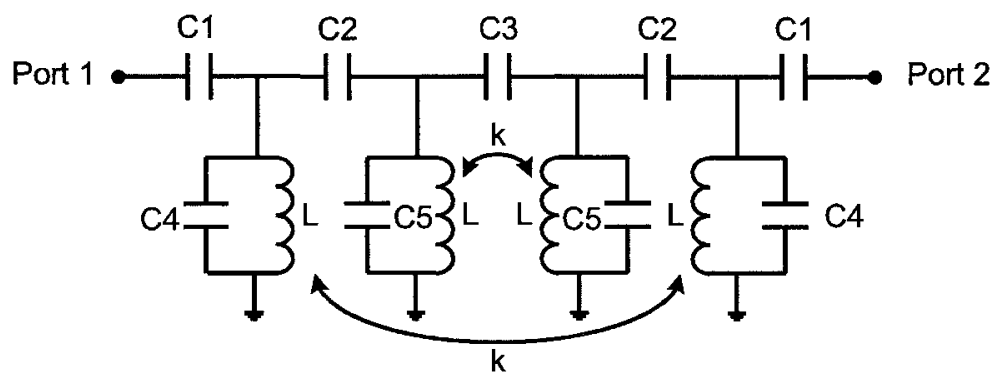

Figure 4.8: Fourth-order filter with inductive coupling.

first and fourth resonators to create a feedback path from input to output. This in turn creates an additional pair of transmission zeros around the passband. To achieve the response shown in Fig. 4.8, the component values listed in Table 4.3 are needed.

Table 4.3: Theoretical Component Values For Improved Fourth-Order Bandpass Filter

\begin{tabular}{|c|c|c|c|c|c|c|}
\hline$L$ & $C 1$ & $C 2$ & $C 3$ & $C 4$ & $C 5$ & $k$ \\
\hline $4.1 \mathrm{nH}$ & $0.66 \mathrm{pF}$ & $0.18 \mathrm{pF}$ & $0.15 \mathrm{pF}$ & $1.77 \mathrm{pF}$ & $2.2 \mathrm{pF}$ & $0.5 \%$ \\
\hline
\end{tabular}

This filter is characterized by a larger resonator inductor compared to the previously described second-order filter, while the required coupling is one order of magnitude smaller. The layout of the filter is done in a "ring" fashion so that the first and 
fourth resonators are adjacent to each other to facilitate coupling. This can be seen in Fig. 4.9.

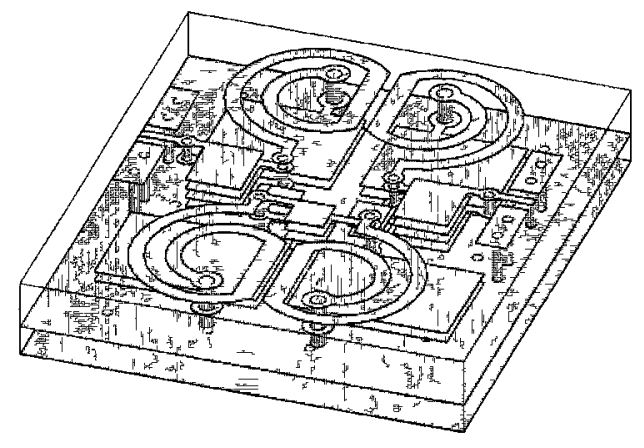

(a)

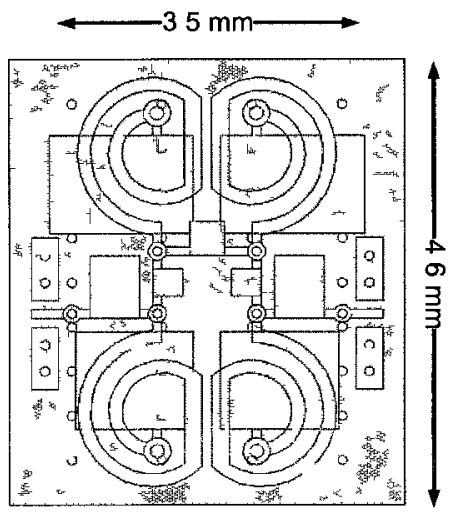

(b)

Figure 4.9: Three dimensional layout of new fourth-order filter (a) oblique view; and (b) top view.

The resonator capacitors ( $C 4$ and $C 5)$ are placed two layers below the inductors to reduce unwanted coupling. As a miniaturization technique, multiple plates are used with the bottom plate being made positive - this implies that the ground plane below effectively acts as a (free) additional capacitor terminal Through these techniques, only 5 layers of LTCC substrate are needed to embed the entıre filter.

Due to the novel layout, the filter core measures only $3.5 \times 4.6 \times 0.485 \mathrm{~mm}^{3}$ or $0.043 \times 0.056 \times 0.006 \lambda_{q}{ }^{3}$, which makes this the smallest reported L-band fourthorder bandpass filter The following sections provide simulated and measured results for the filter as a whole and its individual passive components 


\subsection{Component Library Creation and Simulated Filter Responses}

After obtaining the optimized component values from a circuit simulator, library creation can begin. Every capacitor and inductor must be designed and simulated individually to synthesize the required values before the filter can be assembled.

\subsubsection{Second-Order Filter}

The shunted parallel resonator determines the center frequency of each filter and its associated insertion loss. As LTCC is a high quality dielectric, the dominant loss mechanism is due to the conductor. Therefore, the inductor becomes the limiting factor in obtaining a low passband insertion loss. The new inductor has already been revealed in Fig. 4.6(a). In the quest for higher Q-factor, two important improvements were introduced compared to the resonator inductors of Chapter 3. Namely, the width of the spiral arms was increased from $100 \mu \mathrm{m}$ to $175 \mu \mathrm{m}$ and the separation from $100 \mu \mathrm{m}$ to $150 \mu \mathrm{m}$. The number of turns stays the same at 1.5. From Chapter 3 , the simulated Q-factor was approximately 80 but the measured result (50) was noticeably lower. The simulated inductance and Q-factor are presented in Fig. 4.10.

The Q-factor at $1.55 \mathrm{GHz}$ is estimated to be 80 as well. However, due to the steps taken to reduce the parasitic losses of the spiral, it's expected that the measured Q-factor will be much closer to this estimated value. The inductance was tuned from $3.55 \mathrm{nH}$ to $3.95 \mathrm{nH}$ to obtain a better filter response. The capacitors were also modeled individually to synthesize the desired values but then were readjusted to obtain a better overall filter response. The largest capacitor for this filter is $C 3$, whose simulated capacitance and Q-factor are displayed in Fig. 4.11. 


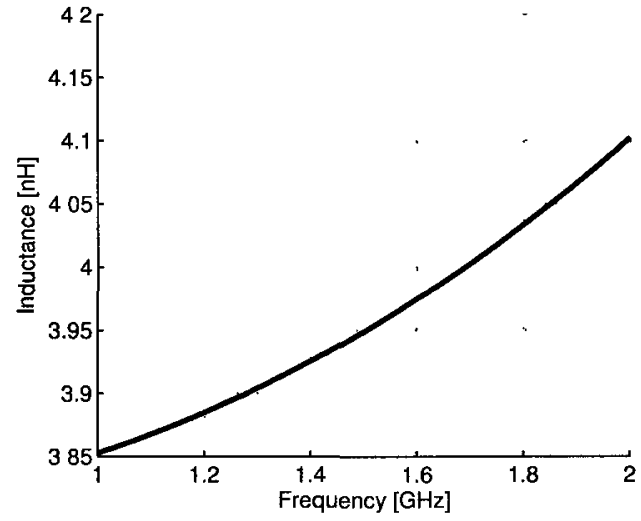

(a)

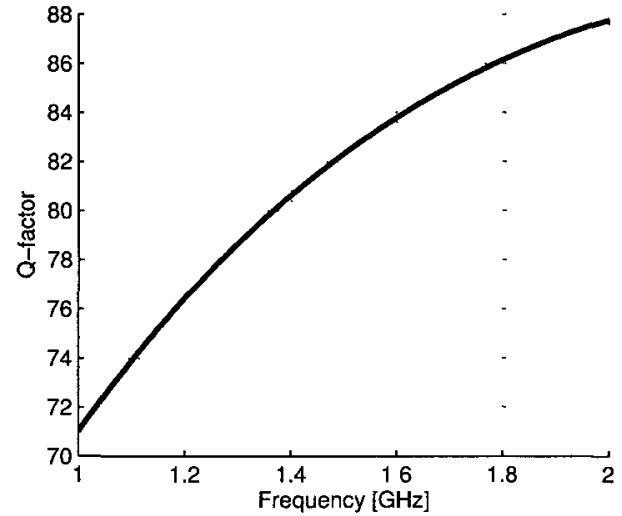

(b)

Figure 4.10: Resonator inductor simulated results for improved second-order filter: (a) inductance; and (b) quality factor.

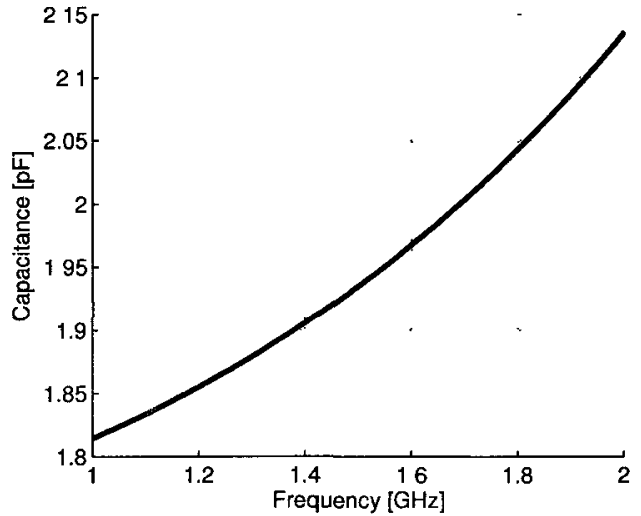

(a)



(b)

Figure 4.11: Resonator capacitor simulated results for improved second-order filter: (a) capacitance; and (b) quality factor. 
As expected, the Q-factor of 250 is much higher for this capacitor than for the inductor, while the self-resonant frequency is safely above $4 \mathrm{GHz}$. The capacitance is $1.93 \mathrm{pF}$ at the center frequency. Although not shown here, the input $(C 1)$ and feedback $(C 2)$ capacitors were designed in the same way. With the completion of the component library, the physical layout of the filter may be contemplated. It was revealed earlier in Fig. 4.7, while the corresponding filter response is presented here in Fig. 4.12.

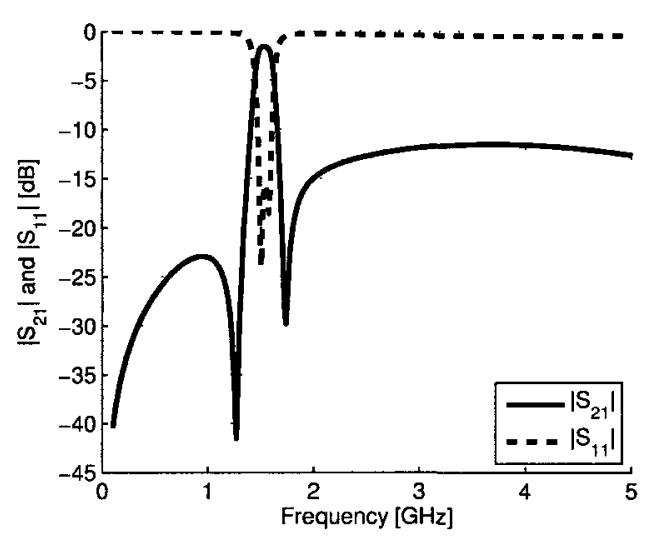

(a)

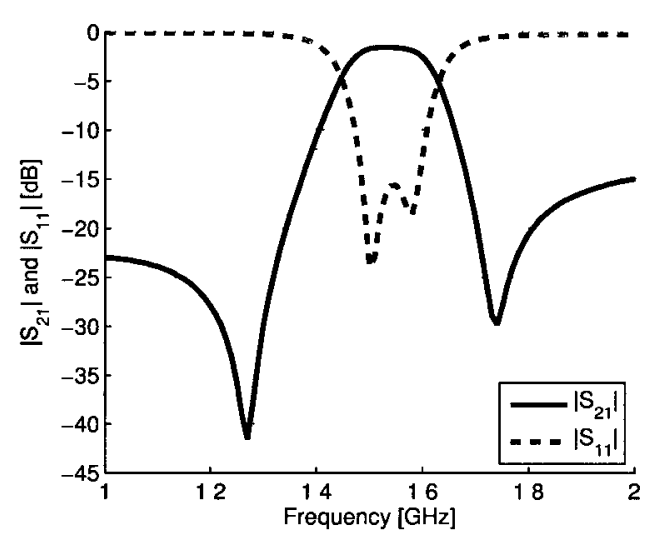

(b)

Figure 4.12: Simulated improved second-order filter response: (a) wideband view; and (b) narrowband view.

The insertion loss of the filter is only $1.8 \mathrm{~dB}$ at the center frequency. The transmission zeros are located at $1270 \mathrm{MHz}$ and $1740 \mathrm{MHz}$, where the attenuation is $41 \mathrm{~dB}$ and $30 \mathrm{~dB}$, respectively. The remaining filter performance parameters are summarized in Table 4.4 .

The $10 \mathrm{~dB}$ return loss bandwidth comfortably covers the required L-band spectrum (1525 MHz to $1575 \mathrm{MHz}$ ) and makes it possible to achieve high yields. Considering the small relative bandwidth of this filter, the maximum in-band insertion loss of 
Table 4.4: Improved Second-Order Filter Simulated Performance

\begin{tabular}{|c|c|c|}
\hline Item & Frequency & Value \\
\hline Center Frequency & {$[1550 \mathrm{MHz}]$} & - \\
\hline Return Loss & {$[1475$ to $1610 \mathrm{MHz}]$} & $135 \mathrm{MHz}$ \\
\hline Insertion Loss & {$[1525$ to $1575 \mathrm{MHz}]$} & $1.8 \mathrm{~dB}(\mathrm{max})$ \\
\hline & {$[100$ to $1330 \mathrm{MHz}]$} & $22 \mathrm{~dB}$ \\
Attenuation & {$[1700$ to $1800 \mathrm{MHz}]$} & $20 \mathrm{~dB}$ \\
& {$[1800$ to $6000 \mathrm{MHz}]$} & $10 \mathrm{~dB}$ \\
First Transmission Zero & {$[1270 \mathrm{MHz}]$} & $41 \mathrm{~dB}$ \\
Second Transmission Zero & {$[1740 \mathrm{MHz}]$} & $30 \mathrm{~dB}$ \\
\hline
\end{tabular}

$1.8 \mathrm{~dB}$ is very low. This helps to keep the system noise figure down when this filter is placed at the front of a receiver chain.

\subsubsection{Fourth-Order Filter}

The improved fourth-order filters' performance is also highly dependent on the quality of its resonator inductors. Slightly more inductance is required compared to the second-order filter ( $4.1 \mathrm{nH}$ vs, $3.55 \mathrm{nH}$ ). Therefore, a larger spiral has been designed that has the same number of turns (1.5). Its shape is also truncated to allow for pairs to be placed closer together (as seen in Fig. 4.9). The simulated inductance and Q-factor are shown in Fig. 4.13.

The Q-factor of approximately 80 is the same as the previous inductors' due to their physical similarity. However, due to its larger size and therefore longer current path, the measured Q-factor will likely be comparatively lower. The inductance was tuned from $4.1 \mathrm{nH}$ to $4.4 \mathrm{nH}$. As presented in Table 4.3, this filter has two different 


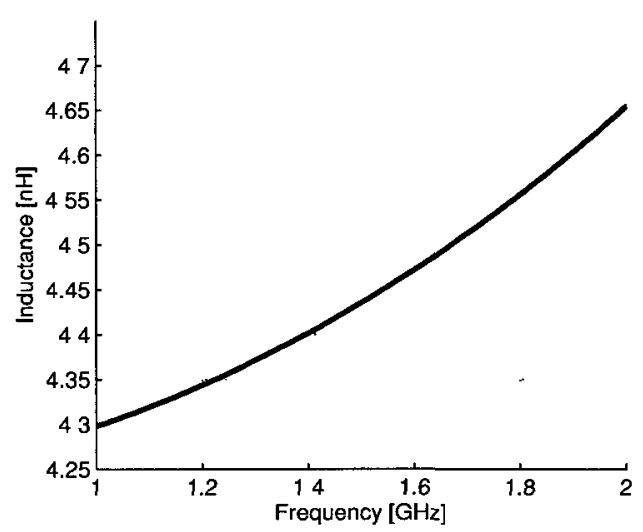

(a)

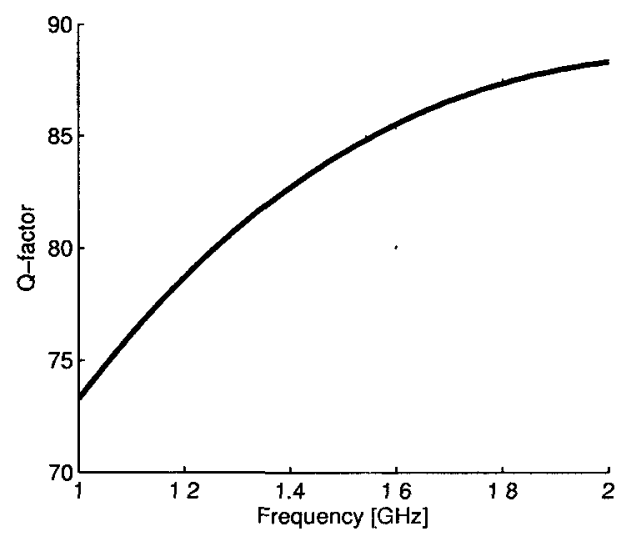

(b)

Figure 4.13: Resonator inductor simulated results for improved fourth-order filter: (a) inductance; and (b) quality factor.

valued resonator capacitors. Both $C 4$ and $C 5$ are implemented with two parallel plates and have optimized capacitances of $2.34 \mathrm{pF}$ and $2.9 \mathrm{pF}$, respectively. The corresponding plots are presented in Fig. 4.14.

The Q-factors for both capacitors are above 200, while their SRFs are safely above $6 \mathrm{GHz}$. The smaller coupling capacitors were designed in the same way. With the component library completed, the filter was assembled according to the "ring" topology described earlier and the final response of Fig. 4.15 was obtained.

The passband is centered around $1.55 \mathrm{GHz}$, where the $10 \mathrm{~dB}$ bandwidth is $130 \mathrm{MHz}$. The high stopband rejection and rapid roll-off at the band edges are attractive features. However, the filters' impressive selectivity is achieved at a high in-band insertion loss of $3.8 \mathrm{~dB}$. All performance characteristics are listed in Table 4.5.

This filter demonstrates that a highly selective response can be achieved with very small area utilization. Even with an insertion loss of $3.8 \mathrm{~dB}$, its high out-ofband rejection and steep roll-off at the passband edges makes this filter potentially 


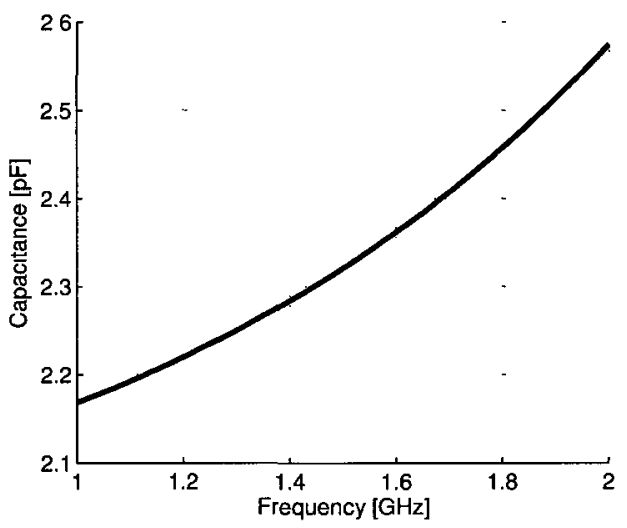

(a)

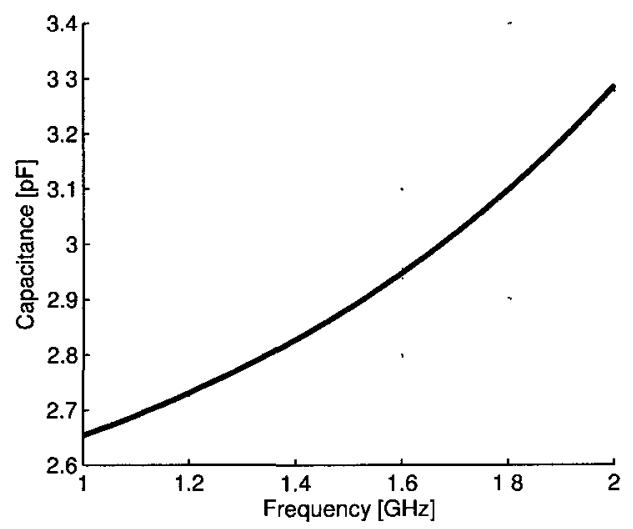

(c)

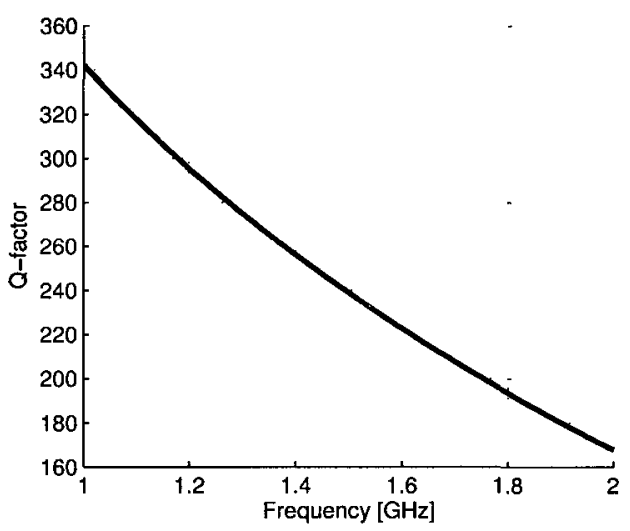

(b)

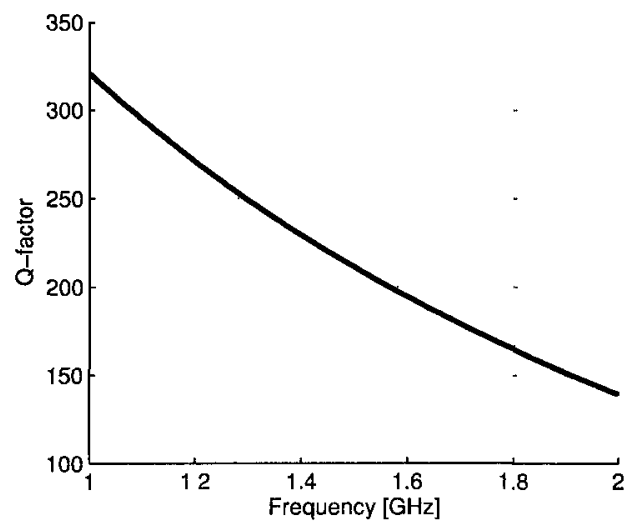

(d)

Figure 4.14: Simulated capacitance and Q-factor for: (a)-(b) $C 4$; and (c)-(d) C5. 


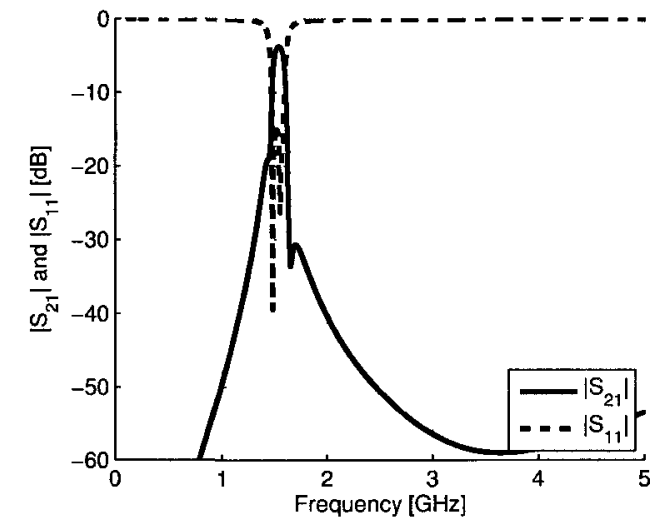

(a)

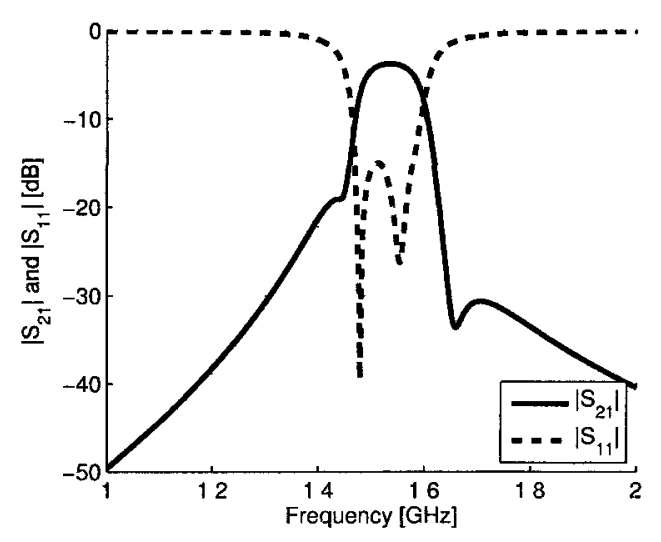

(b)

Figure 4.15: Simulated improved fourth-order filter response: (a) wideband view; and (b) narrowband view.

Table 4.5: Improved Fourth-Order Filter Simulated Performance

\begin{tabular}{|c|c|c|}
\hline Item & Frequency & Value \\
\hline Center Frequency & {$[1550 \mathrm{MHz}]$} & - \\
\hline Return Loss & {$[1460$ to $1590 \mathrm{MHz}]$} & $130 \mathrm{MHz}$ \\
\hline Insertion Loss & {$[1525$ to $1575 \mathrm{MHz}]$} & $3.8 \mathrm{~dB}(\mathrm{max})$ \\
\hline Attenuation & {$[100$ to $1415 \mathrm{MHz}]$} & $20 \mathrm{~dB}$ \\
& {$[1650$ to $6000 \mathrm{MHz}]$} & $20 \mathrm{~dB}$ \\
First Transmission Zero & {$[2000$ to $6000 \mathrm{MHz}]$} & $40 \mathrm{~dB}$ \\
Second Transmission Zero & {$[1442 \mathrm{MHz}]$} & $20 \mathrm{~dB}$ \\
\end{tabular}


attractive for noisy environments with strong interferer signals.

\subsection{Characterization of Improved Filters}

Prototypes of the filters described above were fabricated at the facilities of VTT Electronics in Oulu, Finland. Upon delivery, the filters were characterized at the Communications Research Center. The measurement setup also included $500 \mu \mathrm{m}$ pitch G-S-G probes. The setup was calibrated to the probe tips using the SOLT technique.

\subsubsection{Second-Order Filter}

This filter occupies seven layers of the LTCC substrate that has 8 in total. Therefore, a finite ground plane was inserted on the first metal layer and tied with multiple vias to the external ground plane. Despite this added complexity, the agreement between measured and simulated responses are excellent. The measured response of the improved second-order filter is compared in Fig. 4.16 with the simulated results. The center frequency and locations of the transmission zeros are almost exactly as predicted. The minimum insertion loss in the passband is $2.2 \mathrm{~dB}$ and thus greater than the predicted value of $1.8 \mathrm{~dB}$. This can be explained by examining the measured results for the resonator inductor displayed in Fig. 4.17.

The measured inductance is $4.12 \mathrm{nH}$ or $4 \%$ greater than predicted in the simulations. This result trends well with the measured filter response that shows a reduced center frequency. Since an increased inductance will lead to a lower operating frequency for the resonators. The measured Q-factor is 70 and $15 \%$ below the simulated value of 83 when a $9^{t h}$ degree polynomial is fitted to the data. This is a significant 


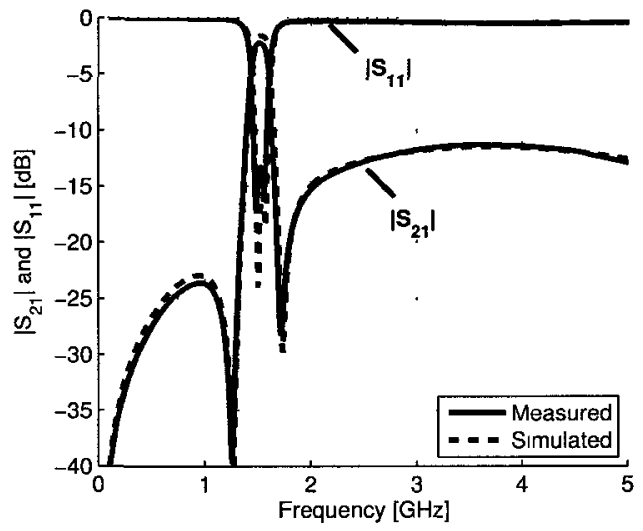

(a)

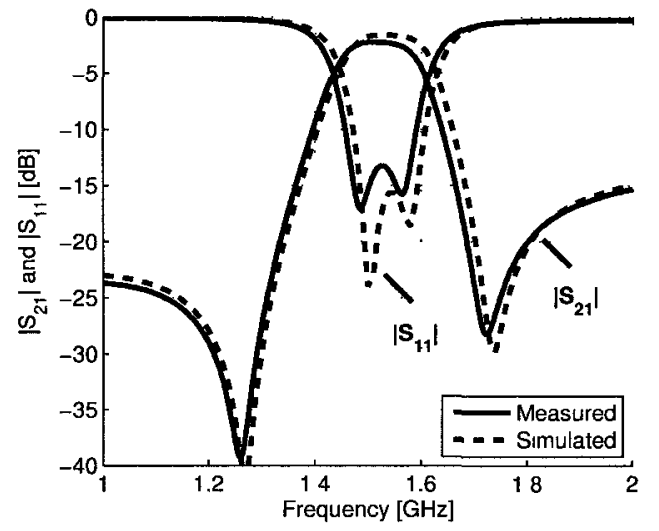

(b)

Figure 4.16: Comparison of measured and simulated second-order filter response: (a) wideband view; and (b) narrowband view.

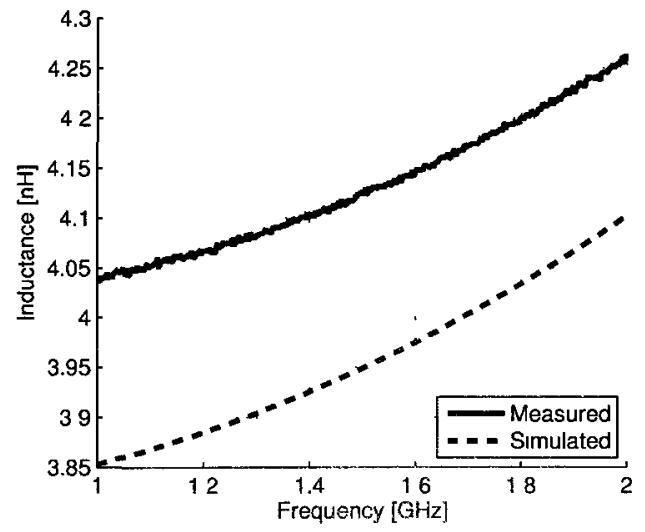

(a)

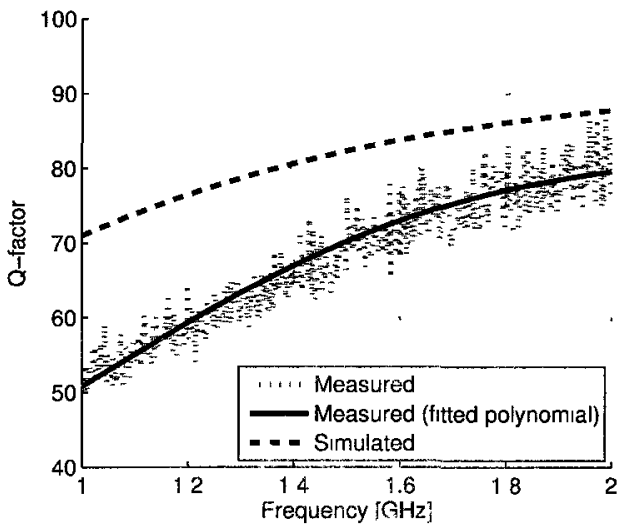

(b)

Figure 4.17: Resonator inductor results comparison: (a) inductance; and (b) Q-factor. 
discrepancy and may be considered the prime cause for the reduction in the minimum insertion loss in the passband for this filter. As was the case in the previous generation filters, the lower Q-factor is due to poor surface roughness for the conducting material. Unfortunately, this is an attribute that is not easily accounted for in the simulator.

The increased inductance of $4 \%$ did not correlate exactly to a $4 \%$ reduction in the center frequency. Instead, only a $1 \%$ reduction was observed. Greater insight into this apparent inconsistency can be gained by examining the measured results for the resonator capacitors presented in Fig. 4.18.

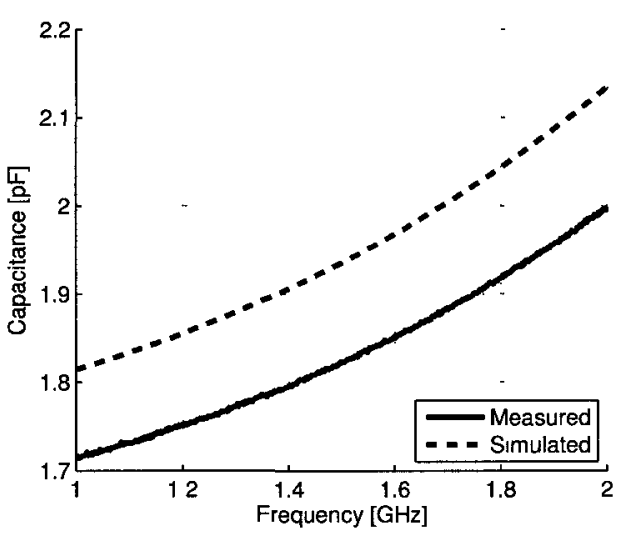

(a)

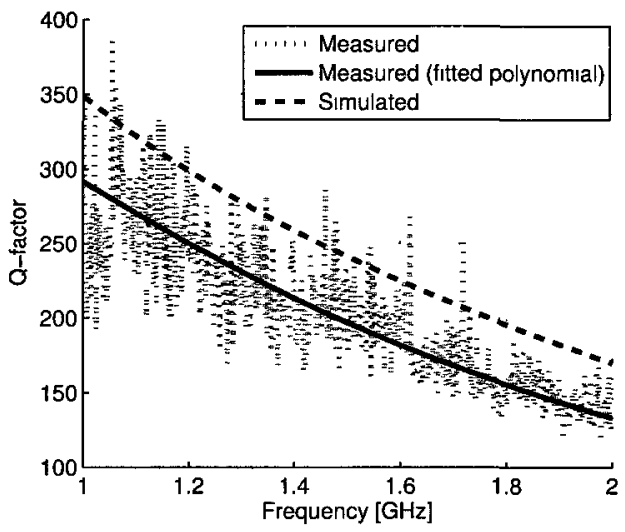

(b)

Figure 4.18: Resonator capacitor results comparison: (a) capacitance; and (b) Qfactor.

The measured capacitance is in fact $1.8 \mathrm{pF}$ or $7 \%$ lower than the expected value of $1.93 \mathrm{pF}$. This adjustment has a counter-balancing effect on the center frequency shift caused by the increased inductance. Therefore, in total, the center frequency stays nearly unchanged. Meanwhile, the Q-factor of this capacitor is 189 (when a $9^{\text {th }}$ degree polynomial is fitted to the data), which is only $8 \%$ lower than the predicted 
figure of 233. The agreement is much better in this case compared to the Q-factor for the inductor because conductor losses play a much smaller role here. Table 4.6 summarizes the differences between measurements and simulations for the resonator components.

Table 4.6: Measurement Summary - Second-Order Filter Components

\begin{tabular}{|c|c|c|}
\hline Item at $\mathbf{1 5 5 0} \mathbf{M H z}$ & $\boldsymbol{C 3}$ & $\boldsymbol{L}$ \\
\hline Simulated Capacitance or Inductance & $1.93 \mathrm{pF}$ & $3.95 \mathrm{nH}$ \\
\hline Measured Capacitance or Inductance & $1.8 \mathrm{pF}$ & $4.12 \mathrm{nH}$ \\
\hline Observed Tolerance & $-7 \%$ & $+4 \%$ \\
\hline \hline Simulated Q-factor & 233 & 83 \\
\hline Measured Q-factor & 189 & 70 \\
\hline Observed Tolerance & $-8 \%$ & $-15 \%$ \\
\hline
\end{tabular}

An overall summary and comparison of results is presented in Table 4.7 for the improved second-order filter.

Despite the small discrepancies noted, the overall agreement between the measured and simulated results is reasonable. The high correlation is a testament to the low tolerance of the LTCC fabrication process and the high degree of accuracy in the electromagnetic simulator employed. The improved second-order filter presented here is the smallest ever reported L-band design, while the inductors employed are of very high quality.

\subsubsection{Fourth-Order Filter}

This design only utilizes 5 of the 8 available LTCC layers. Therefore a partial ground plane was inserted three layers above the main ground plane and tied to it with 
Table 4.7: Results Comparison - Improved Second-Order Filter

\begin{tabular}{|c|c|c|c|}
\hline Item & Frequency & Simulated & Measured \\
\hline Center Frequency & {$[1550 \mathrm{MHz}]$} & - & {$[1524 \mathrm{MHz}]$} \\
\hline Return Loss & {$[1475$ to $1610 \mathrm{MHz}]$} & $135 \mathrm{MHz}$ & $133 \mathrm{MHz}$ \\
& & & {$[1458$ to $1591 \mathrm{MHz}]$} \\
\hline Insertion Loss & {$[1525$ to $1575 \mathrm{MHz}]$} & $1.8 \mathrm{~dB}(\max )$ & $2.2 \mathrm{~dB}(\mathrm{max})$ \\
\hline & {$[100$ to $1330 \mathrm{MHz}]$} & $22 \mathrm{~dB}$ & $23 \mathrm{~dB}$ \\
Attenuation & {$[1700$ to $1800 \mathrm{MHz}]$} & $20 \mathrm{~dB}$ & $20 \mathrm{~dB}$ \\
& {$[1800$ to $6000 \mathrm{MHz}]$} & $10 \mathrm{~dB}$ & $11 \mathrm{~dB}$ \\
First Transmission Zero & {$[1270 \mathrm{MHz}]$} & $41 \mathrm{~dB}$ & $39 \mathrm{~dB} @ 1261 \mathrm{MHz}$ \\
Second Transmission Zero & {$[1740 \mathrm{MHz}]$} & $30 \mathrm{~dB}$ & $28 \mathrm{~dB} @ 1724 \mathrm{MHz}$ \\
\hline
\end{tabular}

numerous vias. The alternative strategy would be to place a 3-layer cavity above the filter but this was deemed more complicated to implement. Nonetheless, these modifications have a small negative impact on the filter performance. The simulated results are joined with the measured data for the improved fourth-order filter in Fig. 4.19 .

As with the second-order filter, the agreement is reasonable. However, there is a small shift of the passband down in frequency and a higher minimum insertion loss. Reviewing the measured results for the resonator inductor in Fig. 4.20 will help understand why.

The measured inductance of $4.9 \mathrm{nH}$ is $0.5 \mathrm{nH}$ or $9 \%$ above the expected value. This variation is similar to the one observed for the second-order filter and acts to lower the center frequency of this filter as well. The simulated Q-factor of 85 is overly optimistic since the observed Q-factor is only 58 (with fitted polynomial) or $22 \%$ lower than expected. This, however, follows what was observed for the second-order 


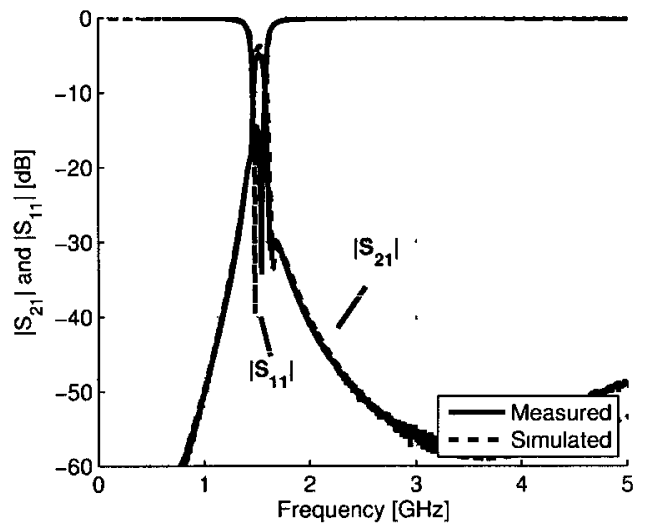

(a)

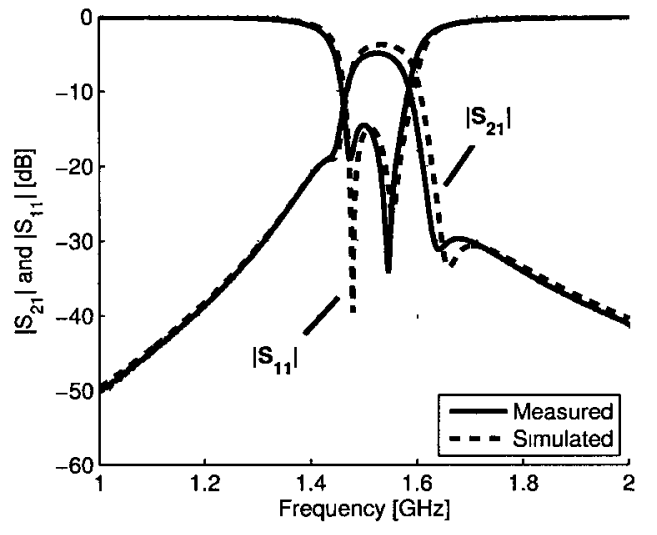

(b)

Figure 4.19: Comparison of measured and simulated fourth-order filter response: (a) wideband view; and (b) narrowband view.

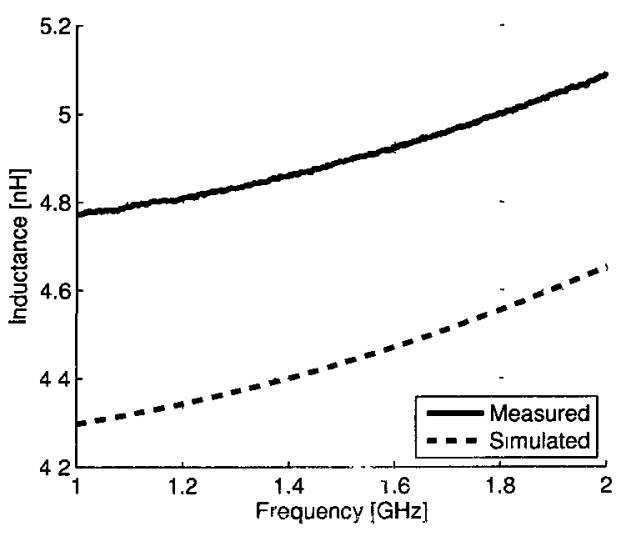

(a)

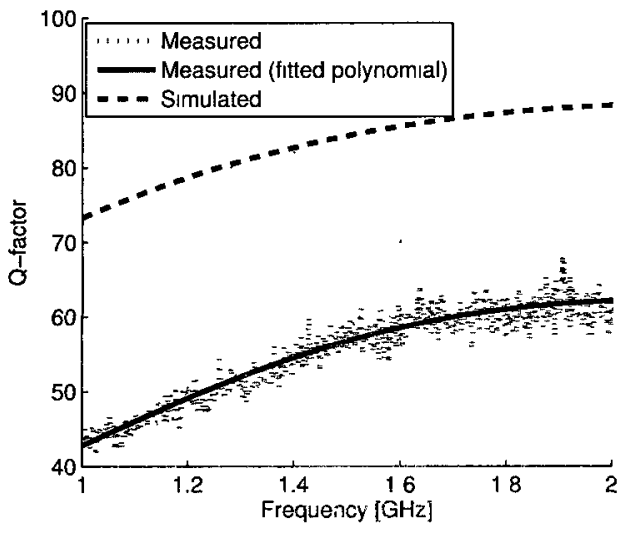

(b)

Figure 4.20: Fourth-order filter resonator results: (a) inductance; and (b) Q-factor. 
filter where the difference is $15 \%$. The cause of this has already been discussed.

As with the previous cases, the measured capacitances of $C 4$ and $C 5$ are lower than expected by about $8 \%$ each. Similarly, their Q-factors are reduced with the largest reduction of $18 \%$ occurring for C5. Reviewing the capacitor measurements, we observe an approximately linear relationship between size and Q-factor tolerance. That is, for a specified percentage increase in capacitance the Q-factor drops by the same amount. The measured results for the resonator capacitors are presented in Fig. 4.21 , while a summary of these results is listed in Table 4.8 .

Table 4.8: Measurement Summary - Fourth-Order Filter Components

\begin{tabular}{|c|c|c|c|}
\hline Item at $\mathbf{1 5 5 0} \mathrm{MHz}$ & $\boldsymbol{C 4}$ & $\boldsymbol{C 5}$ & $\boldsymbol{L}$ \\
\hline Simulated Capacitance or Inductance & $2.341 \mathrm{pF}$ & $2.916 \mathrm{pF}$ & $4.4 \mathrm{nH}$ \\
\hline Measured Capacitance or Inductance & $2.185 \mathrm{pF}$ & $2.664 \mathrm{pF}$ & $4.9 \mathrm{nH}$ \\
\hline Observed Tolerance & $-7 \%$ & $-8 \%$ & $+9 \%$ \\
\hline \hline Simulated Q-factor & 230.8 & 202 & 85 \\
\hline Measured Q-factor & 191.9 & 166 & 58 \\
\hline Observed Tolerance & $-9 \%$ & $-18 \%$ & $-22 \%$ \\
\hline
\end{tabular}

An overall summary and comparison of results is presented in Table 4.9 for the improved fourth-order filter.

The results show once again that the variations in inductances and capacitances oppose each other to make the overall effect negligible. The reduced inductor Q-factor does have a noticeable impact on the minimum insertion loss. Instead of an expected $3.8 \mathrm{~dB}$ loss, the measured result is $4.92 \mathrm{~dB}$. The most direct way to improve these figures is to design even higher quality inductors. 


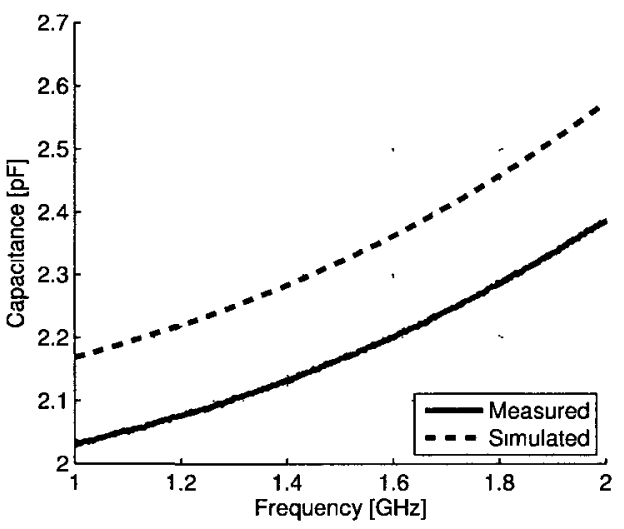

(a)

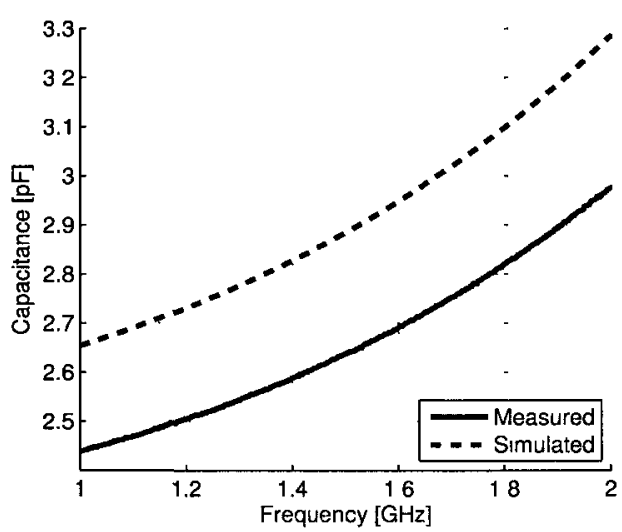

(c)

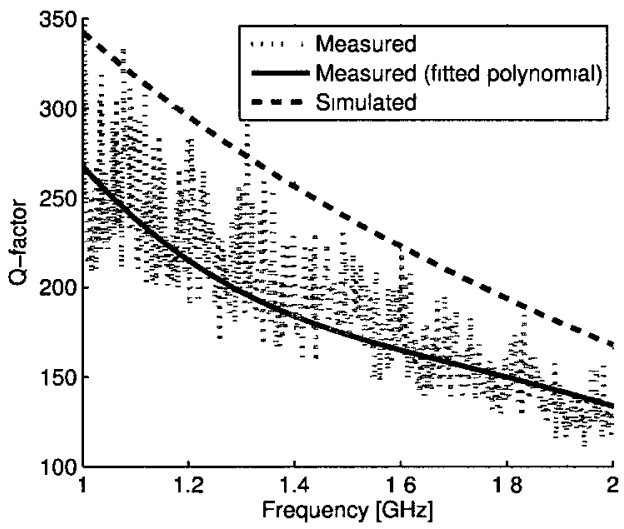

(b)

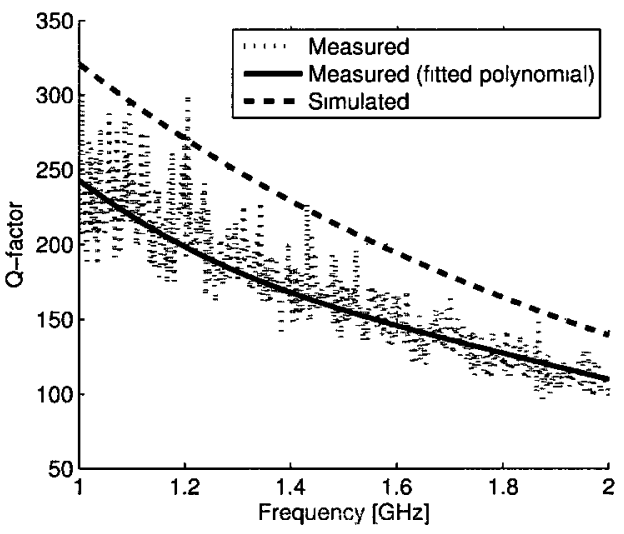

(d)

Figure 4.21: Comparison of capacitance and Q-factor for: (a)-(b) C4; and (c)-(d) C5. 
Table 4.9: Results Comparison - Improved Fourth-Order Filter

\begin{tabular}{|c|c|c|c|}
\hline Item & Frequency & Simulated & Measured \\
\hline Center Frequency & {$[1550 \mathrm{MHz}]$} & - & {$[1521 \mathrm{MHz}]$} \\
\hline Return Loss & {$[1460$ to $1590 \mathrm{MHz}]$} & $130 \mathrm{MHz}$ & $130 \mathrm{MHz}$ \\
\hline Insertion Loss & {$[1525$ to $1575 \mathrm{MHz}]$} & $3.8 \mathrm{~dB}(\max )$ & $4.92 \mathrm{~dB}(\mathrm{max})$ \\
\hline & {$[100$ to $1415 \mathrm{MHz}]$} & $20 \mathrm{~dB}$ & $20 \mathrm{~dB}$ \\
Attenuation & {$[1650$ to $6000 \mathrm{MHz}]$} & $20 \mathrm{~dB}$ & $20 \mathrm{~dB}$ \\
& {$[2000$ to $6000 \mathrm{MHz}]$} & $40 \mathrm{~dB}$ & $30 \mathrm{~dB}$ \\
First Transmission Zero & {$[1442 \mathrm{MHz}]$} & $20 \mathrm{~dB}$ & $18 \mathrm{~dB} @ 1439 \mathrm{MHz}$ \\
Second Transmission Zero & {$[1660 \mathrm{MHz}]$} & $33 \mathrm{~dB}$ & $31 \mathrm{~dB} @ 1639 \mathrm{MHz}$ \\
\hline
\end{tabular}

\subsection{Comments on Simulation-Measurement}

\section{Agreement}

The same fabrication process and substrate material was employed in the realization of the designs described in Chapter 3 and 4 . However, the agreement between simulations and measurements is noticeably better in the latter and can be attributed to three important changes pertaining to the material parameters:

1. Relative permittivity adjustment from 5.9 to 5.7 .

2. Layer thickness estimate changed from $100 \mu \mathrm{m}$ to $97 \mu \mathrm{m}$.

3. Addition of gold plating on exposed metal layers.

These changes are based, in part, on the post-simulation results obtained in Chapter 3. The simulation models were made to reflect these changes and the measured results prove that they are indeed better estimates of the substrate material characteristics. 
The gold plating is a post-processing operation that has two main benefits: i) lower surface roughness and $i$ ) lower ohmic losses in the probe-to-pad contact.

\subsection{Summary and Contribution}

This chapter has presented two improved bandpass filters. The goal was to better the results of chapter 3 in terms of in-band insertion loss and overall size. In both cases, the new filters have achieved these goals. The second-order design has an inband insertion loss $0.6 \mathrm{~dB}$ lower than the previous version, while at the same time occupying $82 \%$ less volume. In reference to other published work, these new filters also compare very favorably in terms of volume occupied as listed in Table 4.10. This table lists related work in the area of narrow-band, single-band, lumped element LTCC filters. To create a fair comparison, the volume occupied by these filters is in terms of guided wavelength $\left(\lambda_{g}\right)$. Otherwise, the differences in center frequency and relative permittivity would not be accounted for. The table is sorted by ascending volume (top - smallest).

Table 4.10: Comparison With Related Work

\begin{tabular}{|c|c|c|c|}
\hline Ref. & $f_{c}(\mathbf{G H z})$ & Dim. in mm and $\left(\lambda_{g}\right)$ & Vol. $\left(\lambda_{g}{ }^{3}\right)$ \\
\hline [This work 1] & 1.55 & $\begin{array}{c}2.6 \times 2.15 \times 0.679 \\
(0.032 \times 0.026 \times 0.008)\end{array}$ & $6.656 \times 10^{-6}$ \\
\hline$[$ This work 2] & 1.55 & $\begin{array}{c}3.5 \times 4.6 \times 0.485 \\
(0.043 \times 0.056 \times 0.006)\end{array}$ & $1.44 \times 10^{-5}$ \\
\hline$[27]$ & 2.45 & $2 \times 1.8 \times 0.67$ & $1.44 \times 10^{-5}$
\end{tabular}


Table 4.10: Comparison With Related Work

\begin{tabular}{|c|c|c|c|}
\hline Ref. & $f_{c}(\mathbf{G H z})$ & $\begin{array}{l}\text { Dim. in mm and }\left(\lambda_{g}\right) \\
(0.046 \times 0.041 \times 0.015)\end{array}$ & Vol. $\left(\lambda_{g}{ }^{3}\right)$ \\
\hline [84] & 1.3 & $\begin{array}{c}7.3 \times 4 \times 0.81 \\
(0.076 \times 0.042 \times 0.008)\end{array}$ & $2.71 \times 10^{-5}$ \\
\hline [85] & 2.4 & $\begin{array}{c}1.6 \times 0.8 \times 0.6 \\
(0.067 \times 0.033 \times 0.025)\end{array}$ & $5.52 \times 10^{-5}$ \\
\hline [82] & 2.5 & $\begin{array}{c}4.4 \times 2 \times 0.54 \\
(0.1 \times 0.047 \times 0.012)\end{array}$ & $5.64 \times 10^{-5}$ \\
\hline$[86]$ & 1.95 & $\begin{array}{c}1.6 \times 0.8 \times 0.52 \\
(0.096 \times 0.048 \times 0.03)\end{array}$ & $1.38 \times 10^{-4}$ \\
\hline$[87]$ & 5 & $\begin{array}{c}1.9 \times 1 \times 0.8 \\
(0.088 \times 0.046 \times 0.037)\end{array}$ & $1.49 \times 10^{-4}$ \\
\hline [74] & 2.45 & $\begin{array}{c}8.3 \times 4.6 \times 0.46 \\
(0.19 \times 0.1 \times 0.01)\end{array}$ & $1.9 \times 10^{-4}$ \\
\hline [88] & 2.55 & $\begin{array}{l}4.52 \times 3.45 \times 1.08 \\
(0.1 \times 0.08 \times 0.025)\end{array}$ & $2 \times 10^{-4}$ \\
\hline [89] & 2.45 & $\begin{array}{c}5.03 \times 3.56 \times 1.08 \\
(0.11 \times 0.08 \times 0.024)\end{array}$ & $2.13 \times 10^{-4}$ \\
\hline$[80]$ & 1.95 & $\begin{array}{c}6.6 \times 6.6 \times 0.836 \\
(0.13 \times 0.13 \times 0.016)\end{array}$ & $2.7 \times 10^{-4}$ \\
\hline$[90]$ & 5 & $5.0 \times 3.5 \times 0.9$ & $1 \times 10^{-3}$ \\
\hline
\end{tabular}


Table 4.10: Comparison With Related Work

\begin{tabular}{|c|c|c|c|}
\hline Ref. & $f_{c}(\mathbf{G H z})$ & $\begin{array}{c}\text { Dim. in mm and }\left(\lambda_{g}\right) \\
(0.2 \times 0.14 \times 0.036)\end{array}$ & Vol. $\left(\lambda_{g}{ }^{3}\right)$ \\
& & \\
\hline
\end{tabular}

Based on this group of work, the new second-order filter (this work 1) is nearly one order of magnitude smaller than any other design. Similarly, the new fourth-order filter (this work 2) is ranked second. The excellent results shown here serve as a significant contribution to the field of L-band filters and passive devices in general. It has been shown also that very good agreement can be achieved between the measured and simulated results. 


\section{Chapter 5}

\section{0-Degree Hybrid Coupler Design}

This chapter continues to pursue the third thesis objective through the design of a 90-degree hybrid coupler. As presented in Fig. 5.1, the hybrid's purpose is twofold: i) to evenly split the RF signal and, ii) provide quadrature outputs since GPS signals are phase-modulated.

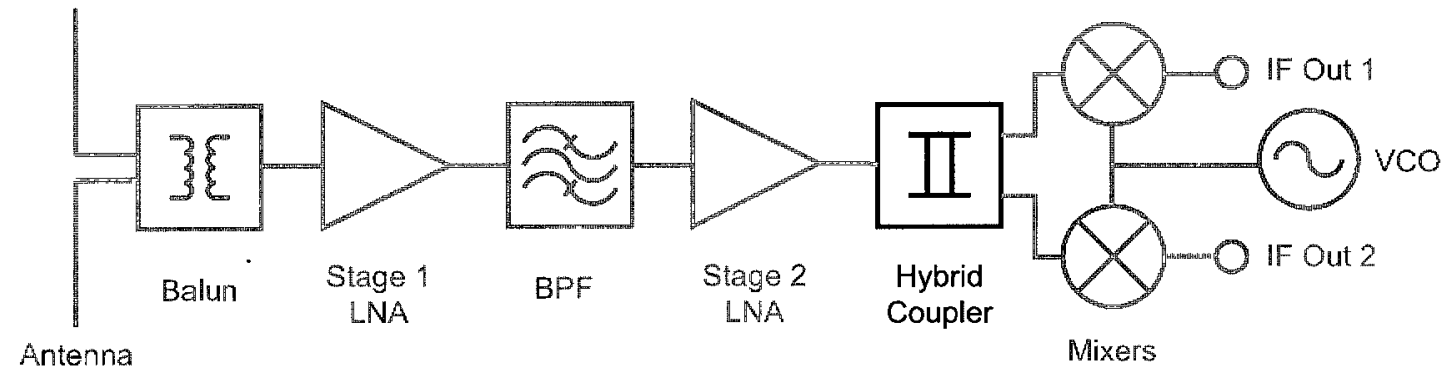

Figure 5.1: Hybrid coupler as part of the complete receiver chain.

Quadrature hybrid couplers are passive devices that perform a $3 \mathrm{~dB}$ split of an input signal and introduce a $90^{\circ}$ phase difference in the outputs. They are commonly used to feed patch antennas for circular polarization. Usually, this device is realized in some type of microstrip or stripline technology [23] [30]. However, at relatively low frequencies such as those in the L-band used for GPS, a conventional microstrip 
hybrid coupler using distributed quarter-wave sections would require several $\mathrm{cm}^{2}$ of area to be realized. As discussed in detail in Chapter 2, several different techniques have been used to reduce the size of couplers. However, they have all been shown to have notable disadvantages. A lumped element approach has been selected as the best approach to implement the coupler here because it reduces the area requirements by $95 \%$ at $1.55 \mathrm{GHz}$ (compared to a microstrip equivalent) with none of the drawbacks of the other techniques [8]. Measured results show insertion losses within $0.65 \mathrm{~dB}$ of the theoretical minimum, an amplitude imbalance of less than $0.18 \mathrm{~dB}$ and a phase difference of 87.4 degrees. Furthermore, the impedance bandwidth is more than sufficient for this application. The following sections will describe the process used to design and optimize the complete coupler.

\subsection{Design Specifications and Circuit Schematic}

The design of the lumped hybrid is based on the classical distributed version shown in Fig. 5.2. The process involves replacing each section of transmission line with equivalent pi lumped networks as presented in Fig. 5.3.

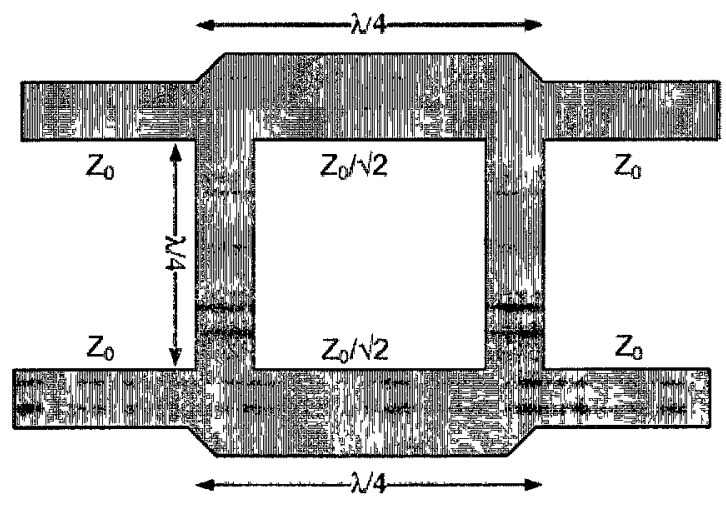

Figure 5.2: Classical distributed coupler layout. 


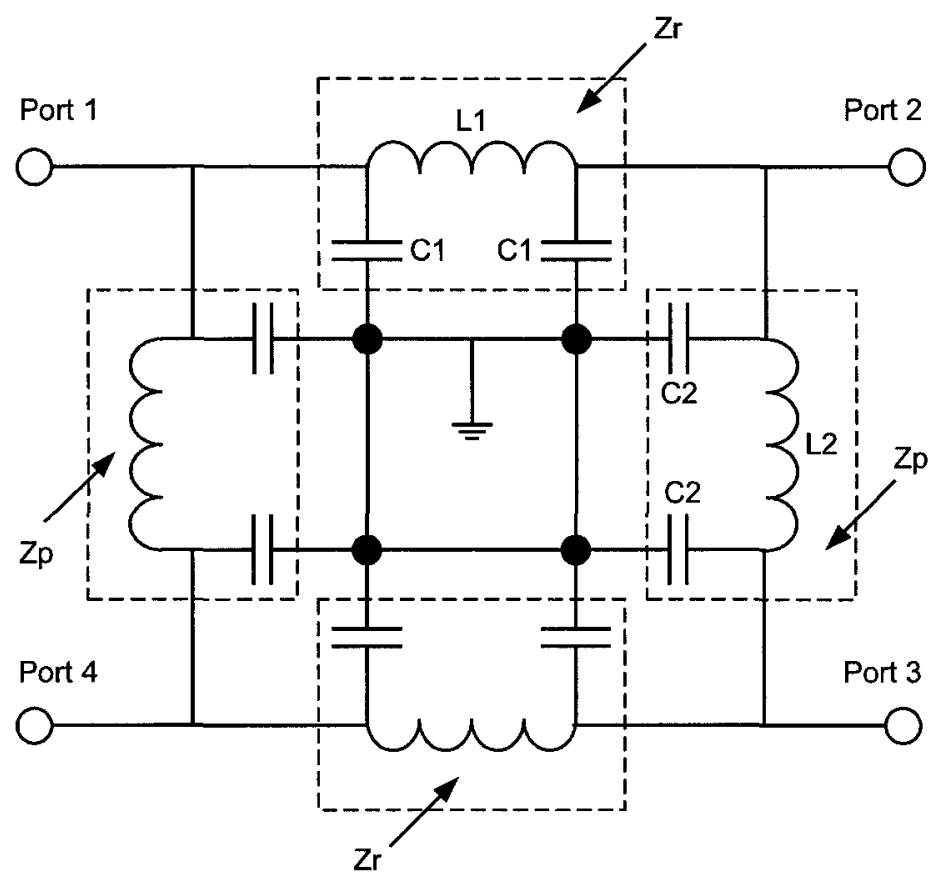

Figure 5.3: Symmetric lumped element schematic for hybrid coupler.

The classical hybrid has a bandwidth of approximately $10 \%$, whereas the lumped equivalent hybrid typical has less because of increased parasitics. This limitation can be mostly avoided by utilizing multiple cascaded pi networks. Here, cascading was not necessary since the bandwidth requirement is small $(<7 \%)$. Equations 5.1 to 5.6 that define the lumped element values can be derived by equating the ABCD-matrix parameters of a pi network to that of a transmission line segment [2].

$$
\begin{gathered}
L 1=\frac{Z_{r} \sin \theta}{\omega} \\
L 2=\frac{Z_{p} \sin \theta}{\omega} \\
C 1=\frac{1}{\omega Z_{r}} \sqrt{\frac{1-\cos \theta}{1+\cos \theta}}
\end{gathered}
$$




$$
\begin{gathered}
C 2=\frac{1}{\omega Z_{p}} \sqrt{\frac{1-\cos \theta}{1+\cos \theta}} \\
Z_{r}=\frac{Z_{0}}{\sqrt{2}} \\
Z_{p}=Z_{0}
\end{gathered}
$$

The coupler is designed to have a centre frequency of $1.55 \mathrm{GHz}$ and $10 \mathrm{~dB}$ bandwidth of over $100 \mathrm{MHz}$, to account for fabrication tolerances. Insertion losses are to be kept as low as possible and an isolation of better than $20 \mathrm{~dB}$ is desired. To calculate the theoretical component values, each line was assumed to have a phase angle, $\theta$, of $90^{\circ}$ and a normalized characteristic impedance, $Z_{0}$, of $50 \Omega$. Performing the calculations results in the values of $3.63 \mathrm{nH}, 5.134 \mathrm{nH}, 3 \mathrm{pF}$ and $2 \mathrm{pF}$ for $L 1, L 2, C 1$ and $C 2$, respectively. These calculations serve as a starting point only. Further tuning and optimization will be considered later. Of note, the methodology used here is useful for coupler designs for frequencies up to C-band range. Beyond which the advantages of using lumped elements are diminished since the wavelength is short enough for distributed elements to be used.

\subsection{Component Library Creation and Simulated Performance}

Having obtained the theoretical component values, modeling of the passive structures to realize these values can begin. The same material system and design procedure is used as before, however, the values to be realized here are larger, especially L2 and C1. An inductance over $5 \mathrm{nH}$ is difficult to achieve using a small planar spiral. An alternative topology is the multi-layer spiral (helix) that uses vertical integration to 
minimize inductor size. Unfortunately, a stacked spiral is expected to have a lower Q-factor because more of the magnetic field is contained in the substrate rather than air, which is less lossy. To implement $C 1$, a stacked parallel plate capacitor is needed. This topology has already been demonstrated in Chapters 3 and 4 in reference to the filter designs. Here, the plates will be enlarged further to achieve the necessary capacitance. The challenge will be to minimize parasitics to ensure that the selfresonant frequency is sufficiently high.

\subsubsection{Inductor Design}

Two different valued inductors are required for this design. As shown in Fig. 5.4, both are implemented as two-port rectangular helical structures. The smaller inductor,

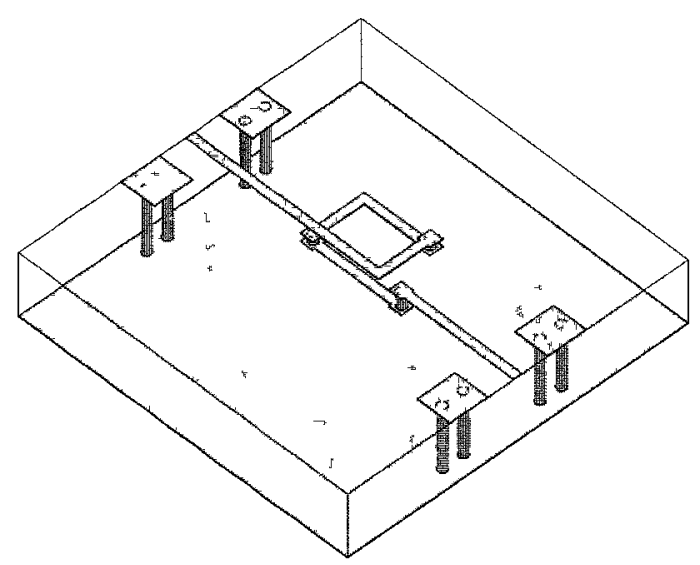

(a)

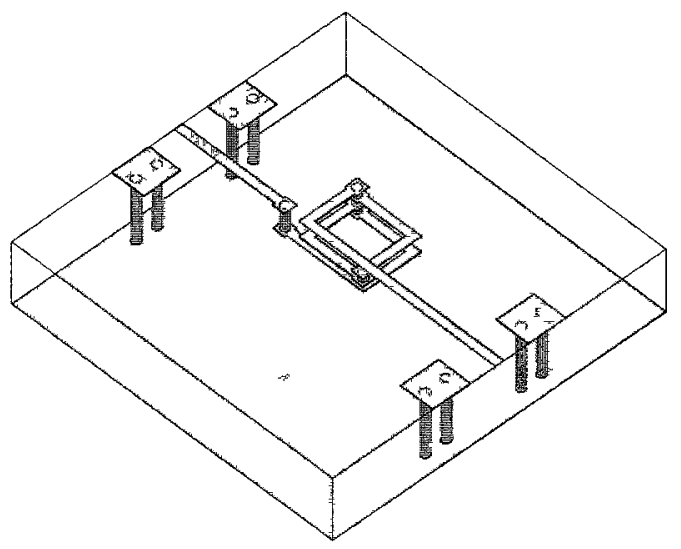

(b)

Figure 5.4: Multilayer spiral inductor structures: (a) $L I$; and (b) $L 2$

$L 1$, uses three metal layers and has $1 \frac{1}{4}$ turns, while the larger, $L 2$, uses four layers and $2 \frac{1}{4}$ turns. Through this vertical integration, their area requirements are kept small: $0.775 \mathrm{~mm} \times 1.25 \mathrm{~mm}$ for $L 1$ and $0.775 \mathrm{~mm} \times 1.15 \mathrm{~mm}$ for L2. Choosing a 
rectangular shape rather than circular also helps minimize the area required. Parasitic capacitances are inherently low for this structure because there are no parallel lines and stacked segments are separated by at least two layers of LTCC. To make the results here comparable with the one-port inductor designs of Chapters 3 and 4 , a conversion to a single-ended input impedance with Equation 5.7 is necessary [91].

$$
Z_{s e}=Z_{11}-\frac{Z_{12} Z_{21}}{Z_{22}}
$$

The previously described equations may now be used to calculate inductance and unloaded Q-factor when $Z_{s e}$ is treated as the one-port input impedance. The square spiral inductor shown here has an advantage compared to the circular planar spirals of Chapter 3 and 4 in regards to its tunability (in simulation). To tune the latter, the radius must be changed resulting in different lengths and widths of the structure. In contrast, the inductance of the square spiral can be tuned by simply adjusting its length, while keeping its width constant. As shown in Fig. 5.5, by varying the length of the inductor (while keeping all other dimensions unchanged) the desired inductance can be chosen. For this four layer spiral, an inductance in the range of $6.5 \mathrm{nH}$ to $10 \mathrm{nH}$ can be selected when the length is varied between $0.3 \mathrm{~mm}$ and $0.9 \mathrm{~mm}$. At the same time, the SRF remains above $3.6 \mathrm{GHz}$. Although not shown, the Q-factor stays at approximately 73 in all cases because of the inductors' inherently low parasitic capacitances. This example has shown an effective method of tuning for this type of inductor that is not shared with circular spiral inductors.

Using the aforementioned tuning technique, $L 1$ and $L 2$ were set to their respective theoretically calculated inductance values. When used as part of the coupler design (to be introduced later), however, their inductances were tuned to $5 \mathrm{nH}$ and 


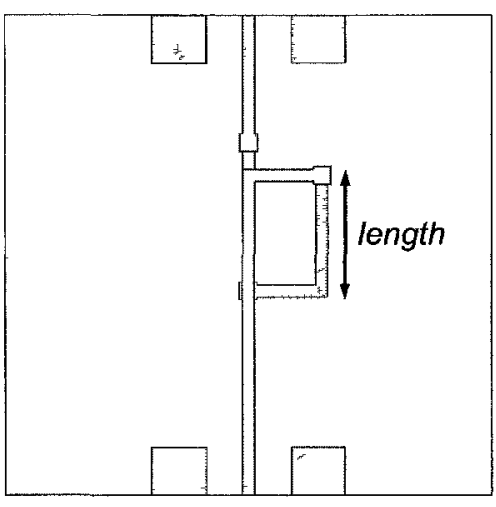

(a)

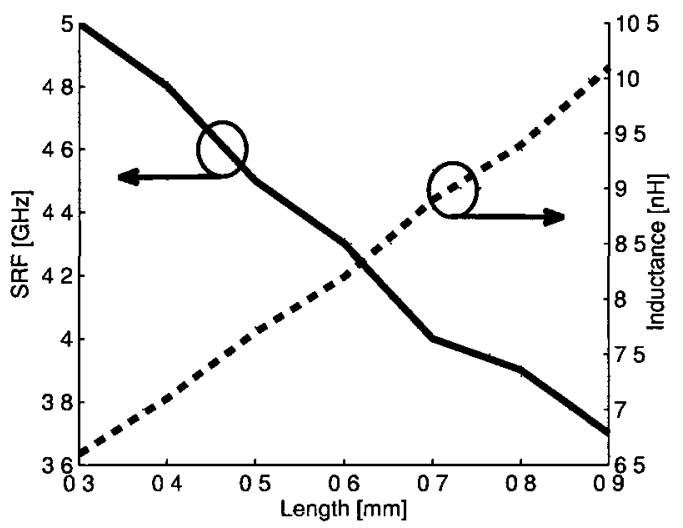

(b)

Figure 5.5: Multilayer spiral inductor structure: (a) length tuning; and (b) its effect on SRF and inductance at $1.55 \mathrm{GHz}$ (all other dimensions unchanged).

$7.5 \mathrm{nH}$ for $L 1$ and $L 2$, respectively, to meet the coupler's performance targets. The corresponding simulated inductance and Q-factor curves are presented in Fig. 5.6.

\subsubsection{Capacitor Design}

In Fig. 5.3, the schematic for the hybrid is presented and shows two capacitors (C1 and C2) on all four corners. On closer inspection, it is evident that these capacitor pairs are connected in parallel and therefore can be combined. This not only simplifies the coupler design but also allows for a smaller layout since some interconnects are made redundant. However, this step will create one overly large capacitor that approaches the limit of what can be achieved with this material system. All elght layers are required to realize the theoretically calculated capacitance of $5 \mathrm{pF}$ $(3 \mathrm{pF}+2 \mathrm{pF})$. The actual capacitor structure used and its dimensions are shown on Fig. 5.7.

The novelty of this capacitor arises from its asymmetrical design that combines 


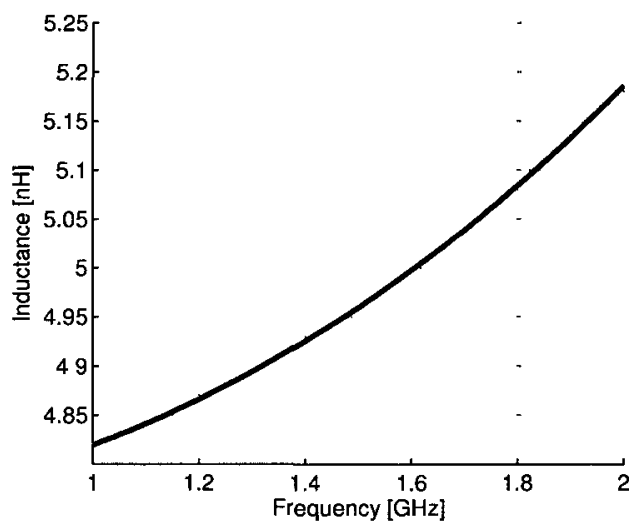

(a)

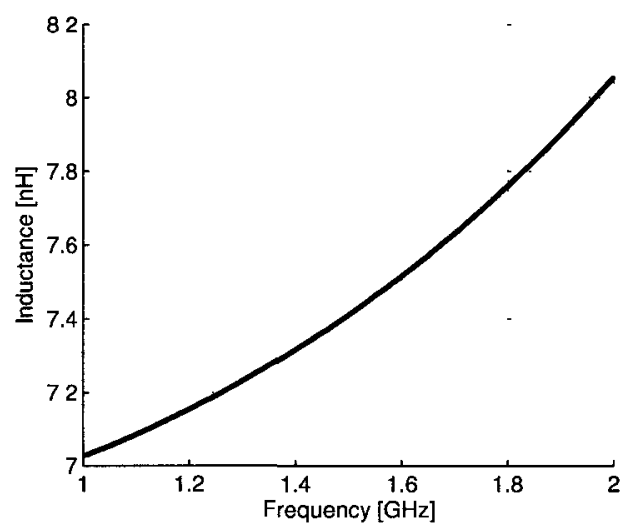

(c)

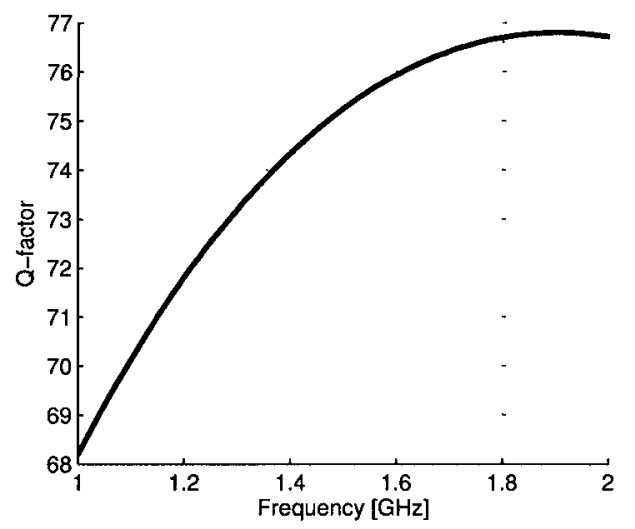

(b)

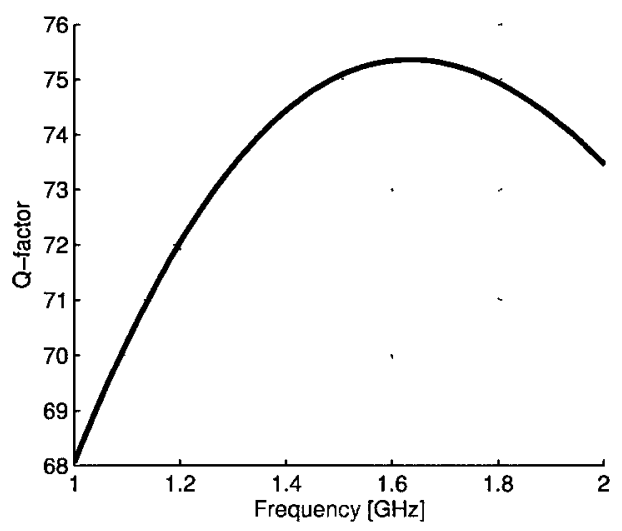

(d)

Figure 5.6: Simulated inductance and Q-factor for: (a)-(b) L1; and (c)-(d) L2. 


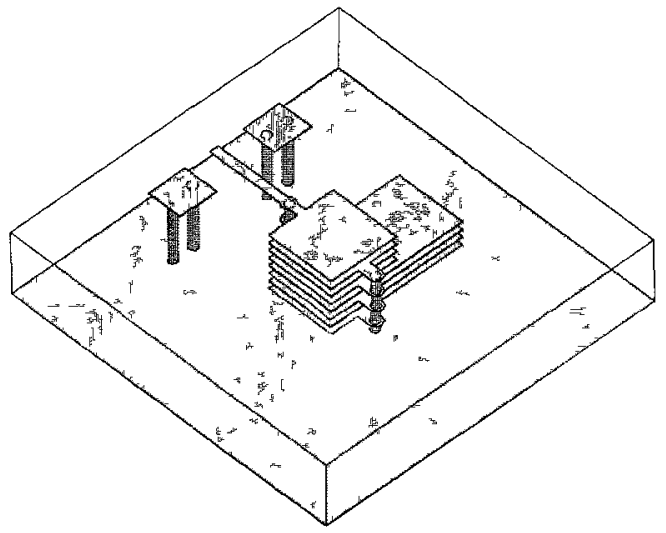

(a)

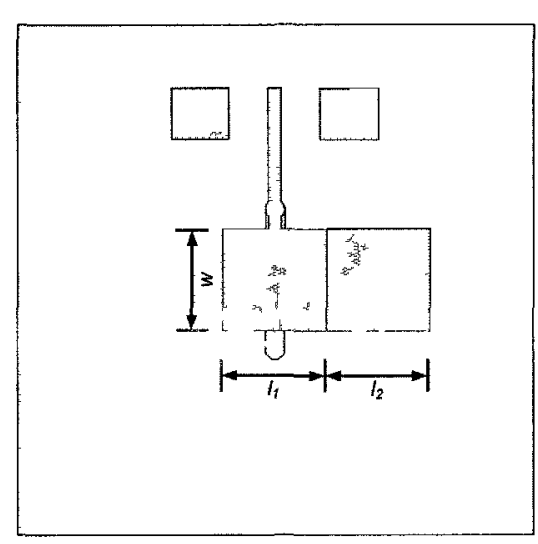

(b)

Figure 5.7: Asymmetric multilayer capacitor structure: (a) oblique view; and (b) dimensions.

differently sized plates. The top four plates have an area equal to $w \times l 1 \mathrm{~mm}^{2}$, while the bottom four have an area equal to $w \times(l 1+l 2) \mathrm{mm}^{2}$. The reason for this asymmetry will become clear when the complete coupler structure is introduced in the next section Suffice it to say, creating high-valued capacitors in LTCC is difficult because of the low relative permittivity (5.7) and large $97 \mu \mathrm{m}$ layer separation. Therefore, large plates are unavoidable and can lead to layout problems when attempting to combine many passive elements. Such is the case here where the adjacent inductors of the coupler are placed nearby and limit the capacitor plate size.

After simulating the completed coupler, it became evident that the capacitance would have to be adjusted higher than the theoretically calculated value of $5 \mathrm{pF}$ If all the layers were to be kept the same size, then the final capacitance of $9.1 \mathrm{pF}$ would not be achieved. In order to solve this problem, the top four layers were made as large as possible without coming too close to the adjacent inductors. Next, the bottom four plates were doubled in area because at those substrate levels there are 
no obstructions (i.e. no metal layers or vias). The final dimensions of the capacitor are: $w=l 1=l \mathscr{2}=0.8 \mathrm{~mm}$. Fig. 5.8 presents the simulated capacitance and Q-factor curves for this capacitor over 1 to $2 \mathrm{GHz}$.

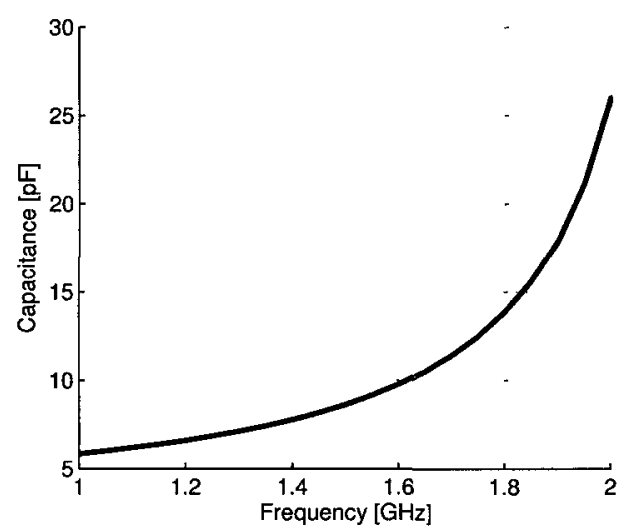

(a)

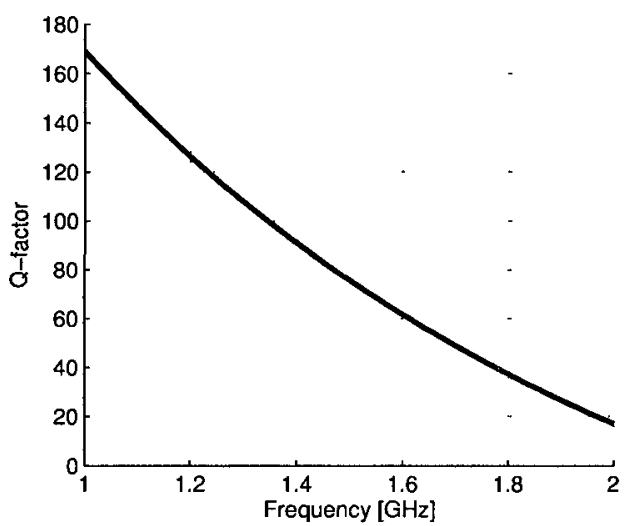

(b)

Figure 5.8: Asymmetric multilayer capacitor simulated performance: (a) capacitance; and (b) Q-factor.

The capacitor Q-factor is only 68, much lower than the smaller capacitors reported earlier. Meanwhile, the SRF is $2.21 \mathrm{GHz}$ and has encroached within a 2:1 ratio for SRF and center frequency. Despite these results, the capacitor is still useful because it is not part of a resonator (where Q-factor is paramount), rather it is simply a component of an artificial transmission line where the actual capacitance value is more important (to achieve the correct characteristic impedance).

\subsection{Coupler Simulated Performance}

A three-dimensional rendering of the coupler, showing embedded metal layers, alongside a top view is presented in Fig. 5.9. 


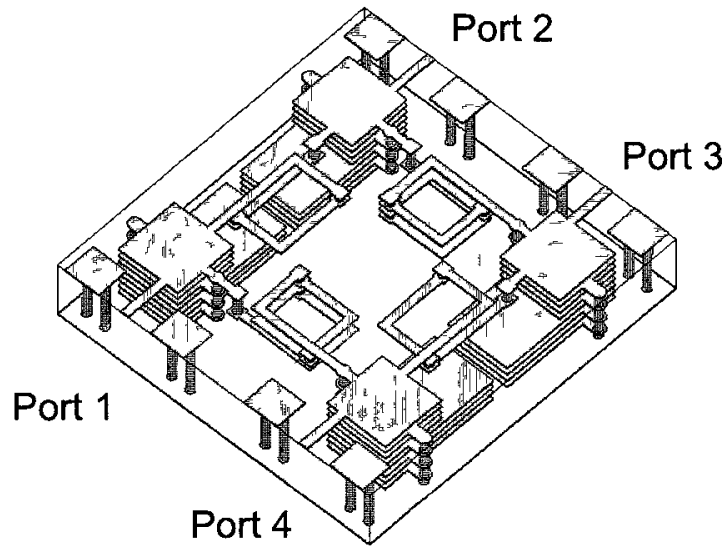

(a)

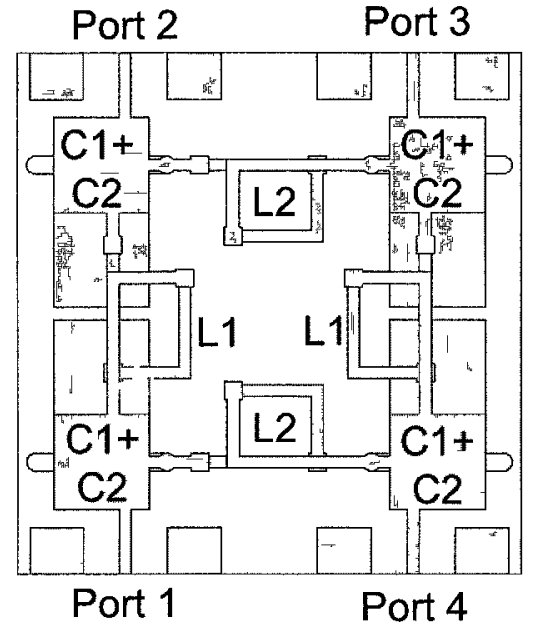

(b)

Figure 5.9: Hybrid coupler structure: (a) three dimensional view; and (b) top view.

The coupler occupies a volume of only $4.4 \times 4.2 \times 0.8 \mathrm{~mm}^{3}$. The material system used here is the same as in Chapter 3 and is described as having eight $97 \mu \mathrm{m}$ layers of FerroA6 LTCC with a relative permittivity of 5.7 and a loss tangent of 0.002 . Silver is the conductor.

Minimizing the coupler size was the primary goal that led to the layout of Fig. 5.9. Several different options were explored and this layout, that has the capacitors placed at the corners and the inductors in between, yielded the smallest part size. The novel layout and packaging of the coupler allows for an extreme degree of miniaturization that is unmatched in the literature. As discussed earlıer, square multı-layer inductors were used as apposed to spiral alternatives because they take better advantage of the three-dimensional capabilities of LTCC. The reason for using asymmetrical capacitors was to take advantage of the empty volume below the inductors. This made it possible to independently size the top four layers (to minimize the overall size of the coupler) and the bottom four layers (to help achieve the desired capacitance). The ports of 
the coupler consist of G-S-G pads that have a pitch of $500 \mu \mathrm{m}$ to be compatible with RF probes of the same geometry.

Initially, the theoretically calculated component values were used for the coupler but simulations showed that the corresponding center frequency was too high. Referring back to the design equations reveals that the center frequency is inversely proportional to the size of $L 1, L 2$, and $C 1$. Therefore, their values were increased until the correct center frequency was achieved. Then fine tuning was carried out to achieve the best amplitude balance and correct phase difference across the outputs. The result of these optimizations is summarized in Table 5.1.

Table 5.1: Component Optimization Results for Lumped Hybrid Coupler

\begin{tabular}{|c|c|c|c|}
\hline Item at $\mathbf{1 5 5 0} \mathbf{~ M H z}$ & $\boldsymbol{L 1}$ & $\boldsymbol{L} \boldsymbol{2}$ & $\boldsymbol{C}$ \\
\hline Theoretical Capacitance or Inductance & $3.63 \mathrm{nH}$ & $5.13 \mathrm{nH}$ & $5 \mathrm{pF}$ \\
\hline Optimized Capacitance or Inductance & $5 \mathrm{nH}$ & $7.5 \mathrm{nH}$ & $9.1 \mathrm{pF}$ \\
\hline Percentage Change & $+38 \%$ & $+46 \%$ & $+82 \%$ \\
\hline
\end{tabular}

The simulated results for the complete hybrid coupler are presented in Fig. 5.10. The center frequency of $1.56 \mathrm{GHz}$ occurs where $S_{11}$ is minimum, while the $10 \mathrm{~dB}$ bandwidth at port one extends from $1.4 \mathrm{GHz}$ to $1.75 \mathrm{GHz}(350 \mathrm{MHz})$. The insertion loss at port two is $3.5 \mathrm{~dB}$ and has a maximum variation of $0.7 \mathrm{~dB}$ over a $100 \mathrm{MHz}$ bandwidth. Similarly, the insertion loss at port three is $3.5 \mathrm{~dB}$ with a maximum variation of $0.1 \mathrm{~dB}$. The amplitude imbalance is less than $0.2 \mathrm{~dB}$ from $1.5 \mathrm{GHz}$ to 1.6 $\mathrm{GHz}$ and the phase difference varies by less than 3 degrees in the same range. These results meet the design specifications given at the beginning of this chapter. 


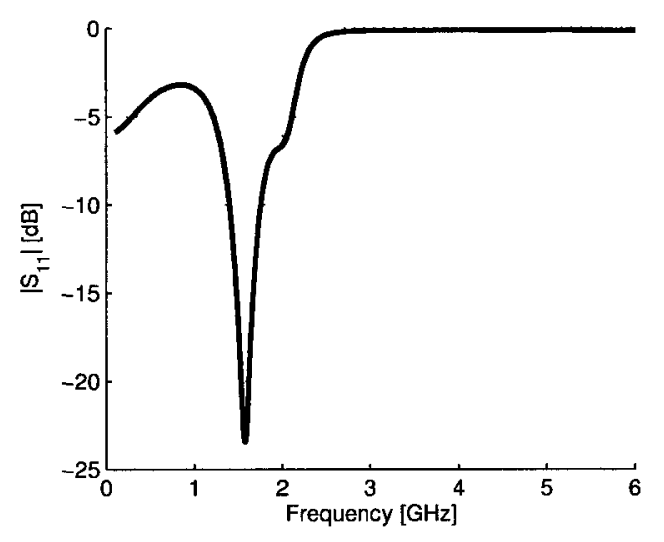

(a) $S_{11}$

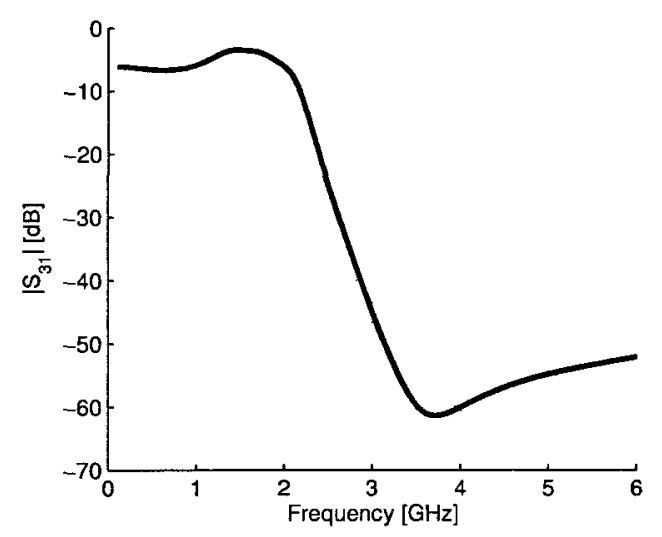

(c) $S_{31}$

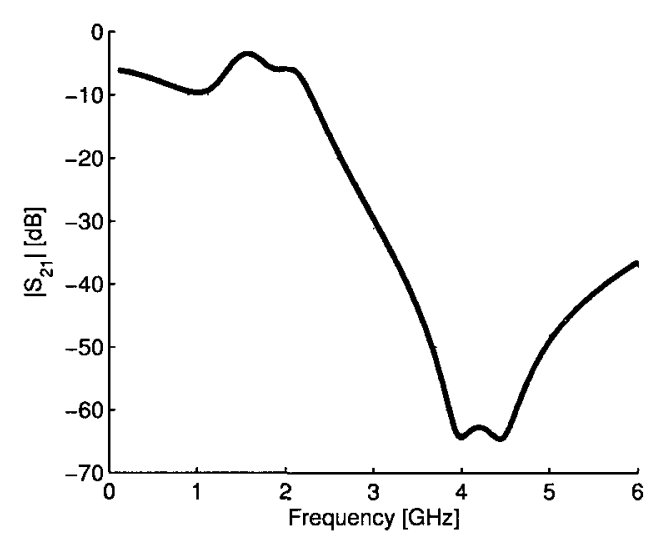

(b) $S_{21}$

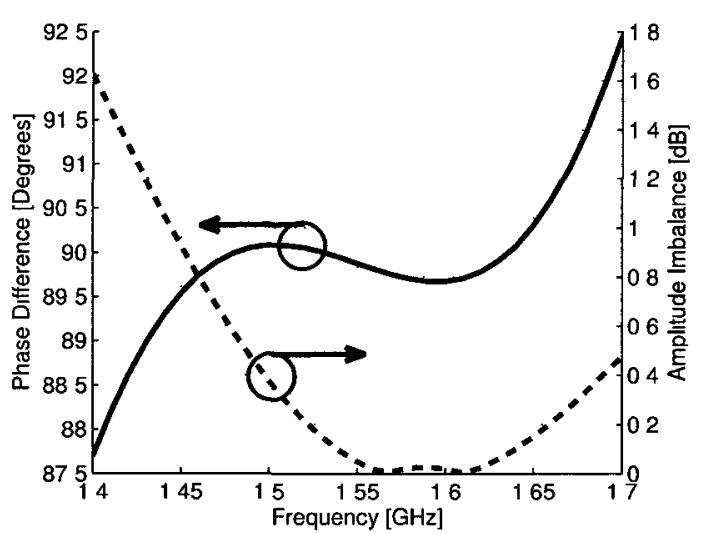

(d) $\Delta \theta$ and arpintude imbalance

Figure 5.10: Simulated coupler performance: (a) $S_{11}$; (b) $S_{21}$; (c) $S_{31}$; and (d) combined $\Delta \theta$ and amplitude imbalance 


\subsection{Hybrid Coupler Characterization}

The fabricated coupler is presented in Fig. 5.11 alongside the test setup. Vector

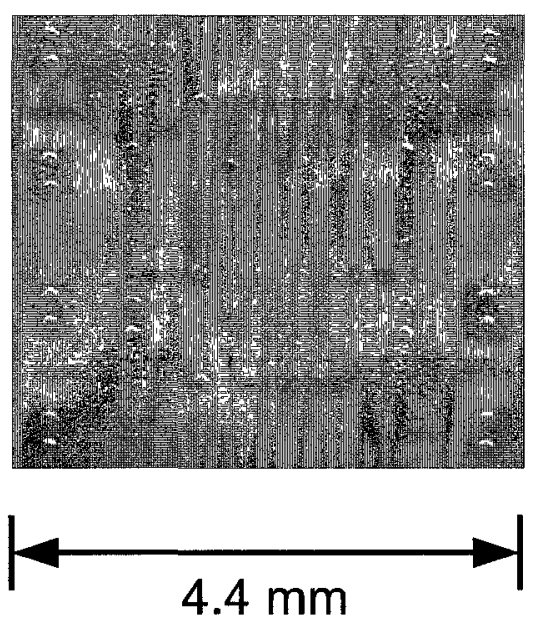

(a)

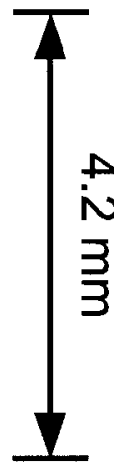

\section{shorting bridges}

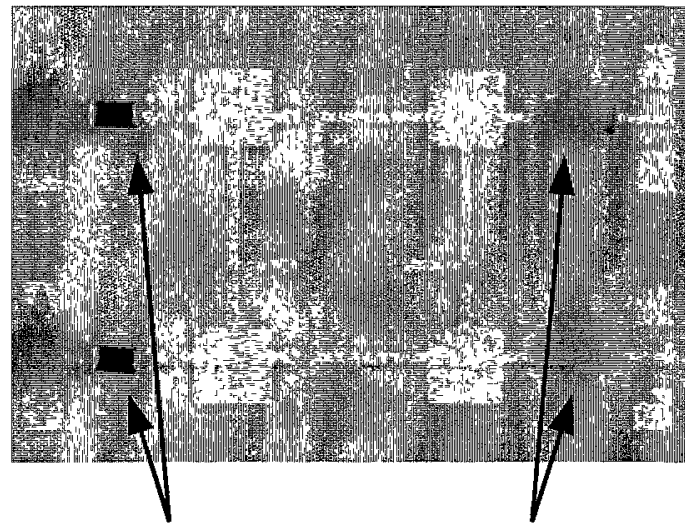

50 Ohm SMT resistors

(b)

Figure 5.11: Fabricated coupler: (a) original component; and (b) modified test setup.

network analyzer measurements were performed after SOLT calibration to the probe tips was carried out. Because a four-port network analyzer was not available, special test layouts were created to measure the device. One such setup is shown in Fig. 5.11(b) and was fabricated alongside the unmodified coupler.

The test layout includes pads for mounting resistors and/or shorts. $50 \Omega \mathrm{SMT}$ resistors are used to load those ports that are not being measured. For the other ports, the gaps are shorted with alumina bridges. All components were affixed with conductive epoxy. To obtain $S_{11}, S_{22}$ and $S_{21}$, ports one and two were prubsil and ports three and four were loaded. Because of the similarity between $S_{11}$ and $S_{22}$, only $S_{11}$ is shown here. $S_{31}$ was measured by probing ports one and three and loading the remainder. The phase difference between the outputs was obtaining by subtracting 
the phases of $S_{21}$ and $S_{31}$ and plotting the absolute value. Similarly, the amplitude imbalance was calculated by subtracting the magnitudes of the insertion losses at ports two and three and taking the absolute value. All these results are contained in Fig. 5.12.

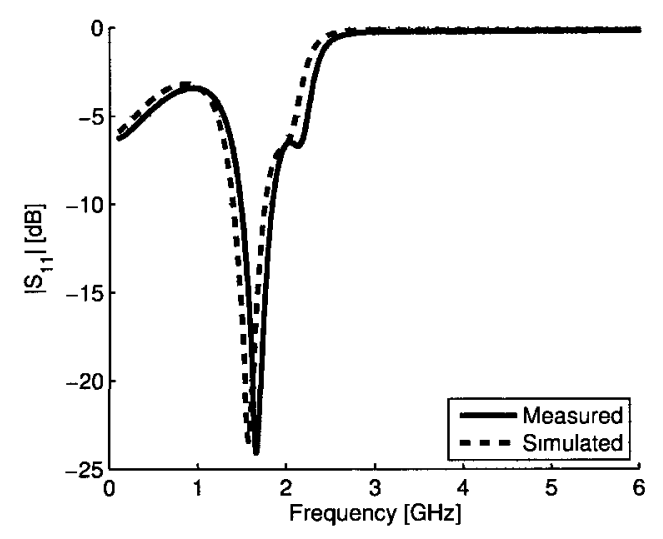

(a) $S_{11}$

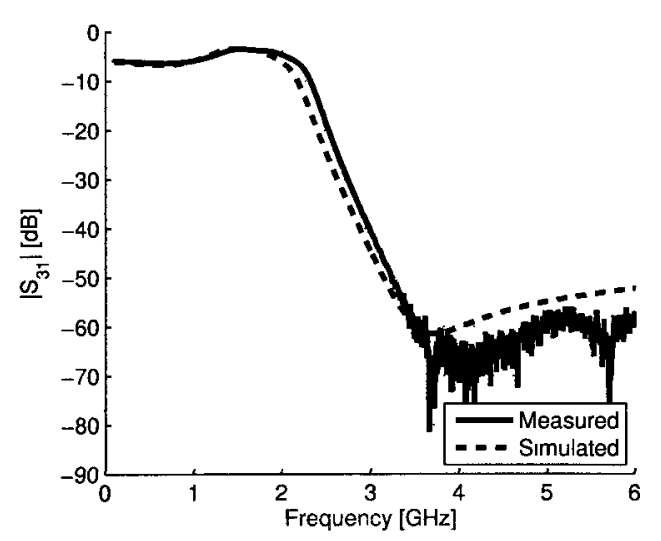

(c) $S_{31}$

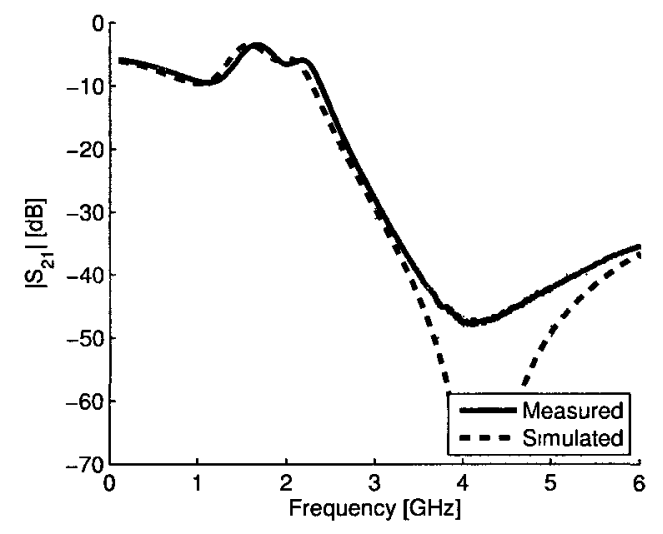

(b) $S_{21}$

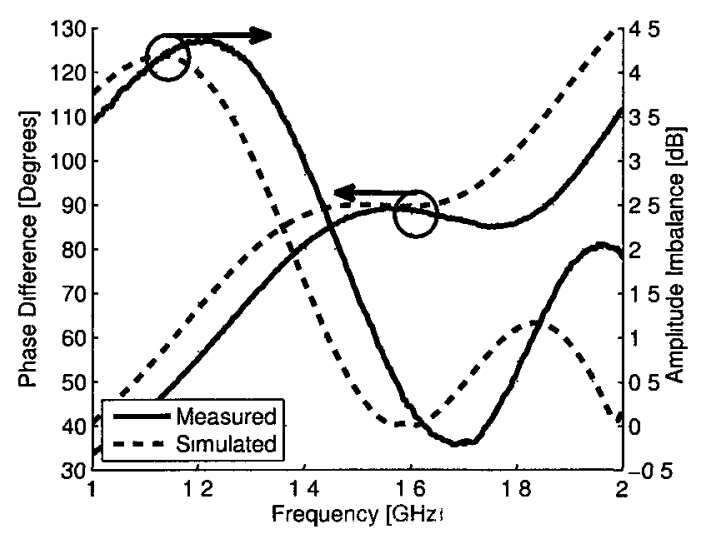

(d) $\Delta \theta$ and amplitude imbalance

Figure 5.12 A comparison of mcasured and sminulazed coupler performance: (a) $S_{11}$; (b) $S_{21} ;$ (c) $S_{31}$, and (d) combined $\Delta \theta$ and amplitude imbalance.

The measured $10 \mathrm{~dB}$ bandwidth at port one is $354 \mathrm{MHz}$ and is centered at $1.66 \mathrm{GHz}$ so the following results are quoted at $1.55 \mathrm{GHz}$. The insertion loss at port two is $3.5 \mathrm{~dB}$ 
and has a maximum variation of $0.2 \mathrm{~dB}$ over the range of $\pm 50 \mathrm{MHz}$. The insertion loss at port three is $3.65 \mathrm{~dB}$ with a maximum variation of $0.1 \mathrm{~dB}$. Fig. 5.12 (d) shows that the amplitude imbalance is less than $\pm 0.2 \mathrm{~dB}$ and the phase difference between the output ports is $87.4 \pm 2.5$ degrees. Although not shown, the isolation between output ports is greater than $20 \mathrm{~dB}$ at the center frequency. The overall agreement is good, however, the measured results show a frequency shift of $+100 \mathrm{MHz}$ or $7 \%$. To attempt to account for this discrepancy, the inductors and capacitor were also measured separately.

First, the capacitor's measured results are compared with simulations in Fig. 5.13. The simulator overestimates the actual capacitance by approximately $13 \%$

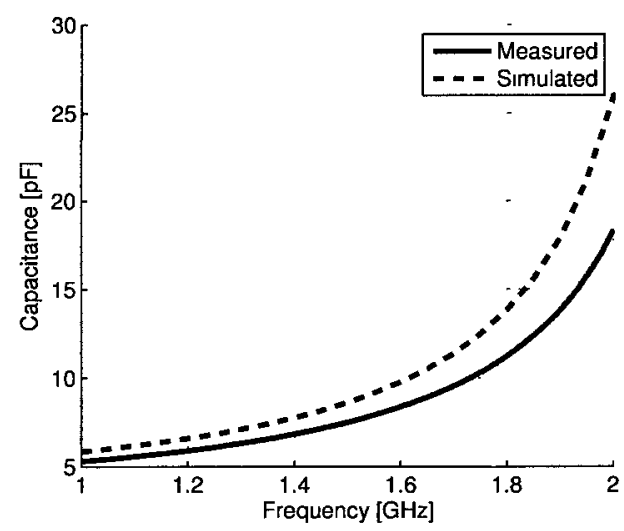

(a)

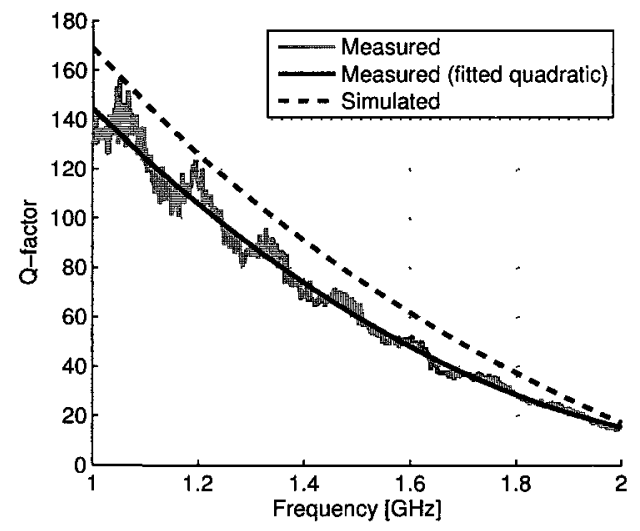

(b)

Figure 5.13: Asymmetric multilayer capacitor measured performance: (a) capacitance; and (b) Q-factor.

at $1.55 \mathrm{GHz}$, being $7.9 \mathrm{pF}$ instead of $9.1 \mathrm{pF}$. The Q-factor is also overestimated but by only $7 \%$, being 57 instead of 68 (when quadratic curve fitting is applied to the measured data). To gain further insight into the coupler's frequency offset, the inductors were also characterized. However, being two port structures, their single ended 
impedance was calculated using Equation 5.7. Fig. 5.14 compares the simulated and measured results directly.

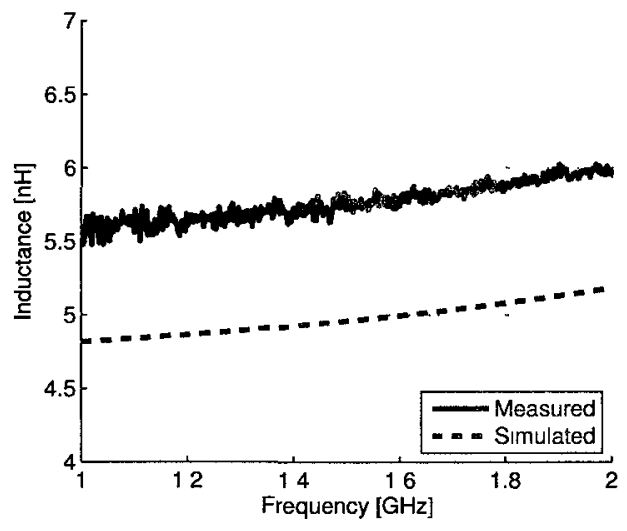

(a)

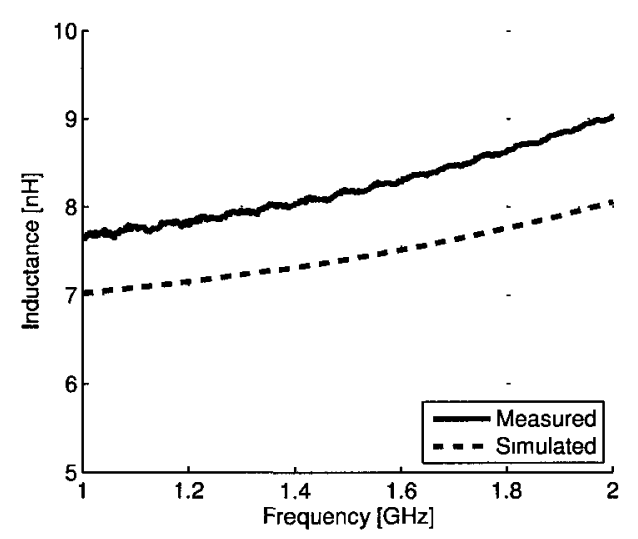

(c)

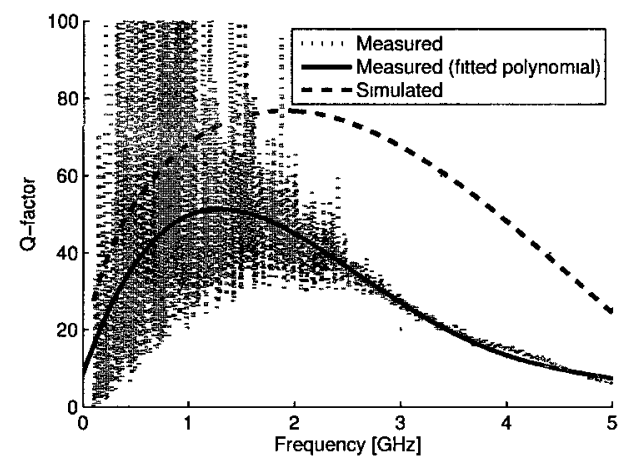

(b)

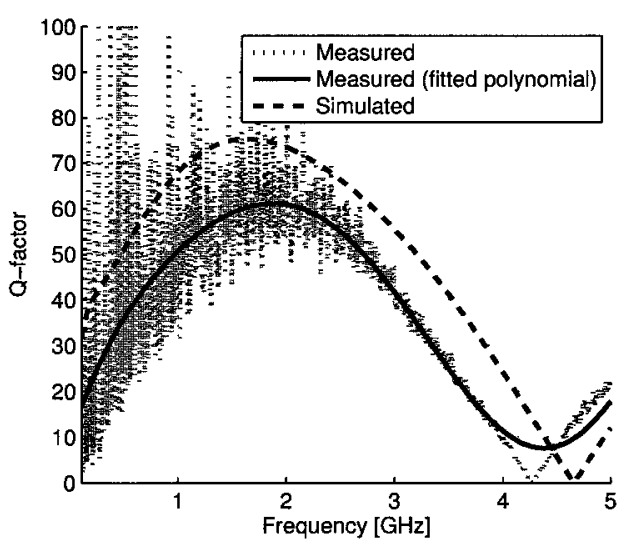

(d)

Figure 5.14: Measured inductance and Q-factor for: (a)-(b) L1; and (c)-(d) L2.

The smaller inductor, $L 1$, displays a measured inductance that is $16 \%$ greater than predicted in simulation $(5.8 \mathrm{nH}$ vs. $5 \mathrm{nH}$, respectively). The corresponding Qfactor is approximately 50 instead of the expected 75. Focusing on the results for L2, we see the same trends where the measured inductance is $10 \%$ too large $(8.2 \mathrm{nH}$ vs. 
$7.5 \mathrm{nH}$ ). This results in a Q-factor of approximately 50 instead of the simulated value of 60. As in Chapters 3 and 4, the inductor Q-factor measurements here also include a significant amount of noise necessitating the application of curve fitting to obtain reliable estimates. The main source of this noise is likely caused by quantization noise of the VNA during calibration. This error in calibration is an inherent factor that in most cases does not cause a problem. However, during the measurement of inductor Q-factors the VNA divides a relatively large number (reactance of input impedance) by a much smaller number (resistance of input impedance) that can generate wildly different values if the resistance is of the same order as the error. Therefore, the noisiest data is observed at lower frequencies where the resistance is smallest $(1 \Omega)$.

A summary of these results along with the calculated tolerances are listed in Table 5.2 .

Table 5.2: Measurement Summary - Hybrid Coupler Components

\begin{tabular}{|c|c|c|c|}
\hline Item at $\mathbf{1 5 5 0} \mathbf{M H z}$ & $\boldsymbol{L 1}$ & $\boldsymbol{L} \boldsymbol{2}$ & $\boldsymbol{C}$ \\
\hline Simulated Capacitance or Inductance & $5 \mathrm{nH}$ & $7.5 \mathrm{nH}$ & $9.4 \mathrm{pF}$ \\
\hline Measured Capacitance or Inductance & $5.8 \mathrm{nH}$ & $8.2 \mathrm{nH}$ & $7.9 \mathrm{pF}$ \\
\hline Observed Tolerance & $+16 \%$ & $+9 \%$ & $-16 \%$ \\
\hline \hline Simulated Q-factor & 60 & 75 & 68 \\
\hline Measured Q-factor & 45 & 55 & 57 \\
\hline Observed Tolerance & $-25 \%$ & $-27 \%$ & $-7 \%$ \\
\hline
\end{tabular}

The discrepancies in these results compared to their respective simulated values are in line with what was observed in Sections 3.6 and 4.5. Since the same fabrication process was used in all cases, the explanations provided in the previous Chapters are also applicable here and are summarized here only. To specifically account for 
the coupler's shifted center frequency, Equations 5.1 to 5.6 can provide some insight. It can be seen that the capacitances and inductances are inversely proportional to frequency. This implies the upwards shift in center frequency may be caused if these values are lower than expected. However, the measured results show the inductances to be in fact larger than required but, conversely, the capacitance to be smaller. These tolerances cancel to some degree but obviously the capacitor tolerance dominates, hence causing the observed shift.

An overall summary and comparison of results is presented in Table 5.3 for the hybrid coupler. Measured results are listed for two different center frequencies; $1.55 \mathrm{GHz}$

Table 5.3: Hybrid Coupler Measurement Summary

\begin{tabular}{|c|c|c|c|}
\hline Item & Simulated & Measured & Measured \\
\hline Center Frequency & $1.55 \mathrm{GHz}$ & $1.55 \mathrm{GHz}$ & $1.66 \mathrm{GHz}$ \\
\hline $10 \mathrm{~dB}$ Return Loss BW at Port 1 & $350 \mathrm{MHz}$ & $354 \mathrm{MHz}$ & $354 \mathrm{MHz}$ \\
\hline$\left|S_{21}\right|$ & $-3.5 \mathrm{~dB}$ & $-4.2 \mathrm{~dB}$ & $-3.5 \mathrm{~dB}$ \\
\hline$\left|S_{31}\right|$ & $-3.5 \mathrm{~dB}$ & $-3.5 \mathrm{~dB}$ & $-3.65 \mathrm{~dB}$ \\
\hline Phase Difference & $90[$ degrees] & $89[$ degrees] & $87.4[$ degrees] \\
\hline$\left|S_{21}\right|$ Variation $[ \pm 50 \mathrm{MHz}]$ & $0.3 \mathrm{~dB}$ & $0.7 \mathrm{~dB}$ & $0.18 \mathrm{~dB}$ \\
\hline$\left|S_{31}\right|$ Variation $[ \pm 50 \mathrm{MHz}]$ & $0.1 \mathrm{~dB}$ & $0.1 \mathrm{~dB}$ & $0.1 \mathrm{~dB}$ \\
\hline Phase Balance $[ \pm 50 \mathrm{MHz}](\max )$ & $0.2[$ degrees] & $1[$ degrees] & $2.5[$ degrees] \\
\hline Amp. Imbalance $[ \pm 50 \mathrm{MHz}](\max )$ & $0.4 \mathrm{~dB}$ & $1.4 \mathrm{~dB}$ & $0.2 \mathrm{~dB}$ \\
\hline
\end{tabular}

and $1.66 \mathrm{GHz}$. The former is provided so that a direct comparison with the simulated data can be made, while the latter is the actual center frequency for the measured device. In both cases the agreement is reasonable. At $1.55 \mathrm{GHz}$ the phase difference and balance is better than at $1.66 \mathrm{GHz}$ but the opposite is true in regards to amplitude 
imbalance.

\subsection{Summary and Contribution}

This chapter has presented a new high-performance hybrid coupler with an extreme degree of miniaturization. The purpose of this device is to create quadrature signals for $\mathrm{I} / \mathrm{Q}$ downconversion and demodulation. It is based on a lumped equivalent model of a conventional hybrid coupler and occupies only $4.4 \times 4.2 \times 0.8 \mathrm{~mm}^{3}$ of a standard low permittivity LTCC. Measured results centered at $1.66 \mathrm{GHz}$ show insertion losses within $0.65 \mathrm{~dB}$ of the theoretical maximum, an amplitude imbalance of less than $0.2 \mathrm{~dB}$ and a phase difference of 87.4 degrees (see Table 5.3 for a complete summary). The specific publication arising from the work contained in this chapter includes one conference paper [8].

The good performance and small size of the device presented here make it suitable for use as a discrete component for various purposes or as an integrated component for SoP applications. To prove its high performance, a comparison with literature was attempted. However, because of the uniqueness of this design comparable work could not be found. Instead commercial components from AVX, Murata, and Johnson Technology were used for comparison in Table 5.4.

The performance of the hybrid coupler presented here compares very favorably to similar commercial products belonging to industry leading companies. In fact, this couplers impedance bandwidth, phase balance, and amplitude balance are superior to the commercial examples given in Table 5.4. This chapter demonstrates the complete fulfillment of the third thesis contribution. 
Table 5.4: Comparison with Commercial Components

\begin{tabular}{|l|c|c|c|c|}
\hline Item & This Work & AVX RF & Murata & Johnson \\
\hline Model No. & N/A & DB0805A1650 & LDC32900M03B-703 & 1950CH15A100 \\
\hline $\begin{array}{l}\text { Center Freq. } \\
\text { [MHz] }\end{array}$ & 1660 & 1542 & 900 & 1950 \\
\hline $\begin{array}{l}\text { Bandwidth - } \\
\text { Input [MHz] }\end{array}$ & 354 & 100 & 200 & 100 \\
\hline $\begin{array}{l}\text { I. Loss [dB] } \\
\text { (max) }\end{array}$ & 0.5 & 0.35 & 0.8 & 0.8 \\
\hline $\begin{array}{l}\text { Phase Bal- } \\
\text { ance [degrees] } \\
\text { (max) }\end{array}$ & 2.5 & 3 & 3 & 3 \\
\hline $\begin{array}{l}\text { Amplitude } \\
\text { Imbalance } \\
\text { [dB] (max) }\end{array}$ & 0.2 & 0.5 & 1 & 1 \\
\hline
\end{tabular}




\section{Chapter 6}

\section{0-Degree Hybrid Coupler (Balun)}

\section{Design}

This chapter represents the partial fulfillment of the fourth thesis objective by presenting the balun, which is a required component of the balun-antenna combination. Its purpose is to convert the balanced antenna output to a single ended feed for the LNA. Its position in the receiver chain is shown in Fig. 6.1. Baluns are usually

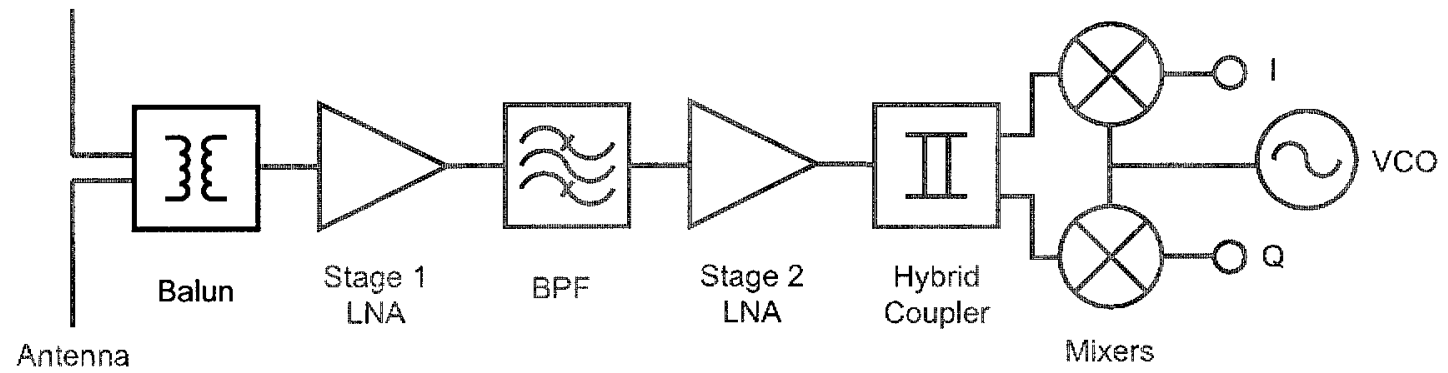

Figure 6.1: Bandpass filter as part of the complete receiver chain.

passive devices used to convert single-ended or unbalanced signals to differential or balanced equivalents. An ideal balun introduces a 180 degree phase shift at its output terminals and also divides the input power equally. Baluns are often placed between 
single ended filters and balanced mixers, amplifiers or antenna feed networks.

The type of balun presented here is also known as a rat-race hybrid, which in turn is a special case of a branch-line coupler whose circumference is an odd multiple of 1.5 $\lambda$. The conventional approach has been to use distributed elements (microstrip, stripline etc...) to implement such a balun. A layout of this classical design is shown in Fig. 6.2. This approach can yield low loss, broadband devices that are simple to design and manufacture [29]. However, due to its electrical length, the size of the balun is often too large to be of practical use except at millimeter frequencies [92] [36].

To aid in miniaturization, lumped element loading has been used to achieve significant size reductions [93] [21]. The tradeoff is a much smaller operating bandwidth. Exotic design techniques that utilize metamaterial lines can, to some degree, extend the operating bandwidth but the component size remains relatively large [94]. With about the same operating bandwidth of the loaded case, further miniaturization is possible if the entire balun is implemented with lumped elements [95]. Simplified networks can be realized with extremely small dimensions but they display even narrower bandwidth [96] or poor phase balance [97]. Moreover, simplified networks make it difficult to optimize the amplitude and phase balance simultaneously due to the reduced number of design variables [98]. Higher order networks can be used to create broadband baluns at the expense of increased insertion loss and component size [99] [100].

Since the primary concerns here are amplitude and phase balance and not broad bandwidth, a first order lumped model is used [95]. Its design is based on theory similar to that of the 90-degree hybrid coupler of Chapter 5. Compared to a conventional microstrip rat-race example, the balun is smaller in area by $98 \%$ at $1.55 \mathrm{GHz}$. Measured results show insertion losses within $0.67 \mathrm{~dB}$ of the theoretical minimum, 
an amplitude imbalance of less than $0.2 \mathrm{~dB}$ and a phase difference of 179.2 degrees. Furthermore, the impedance bandwidth is over $500 \mathrm{MHz}$ and more than sufficient for this application. The following sections will describe the process used to design and optimize the complete balun. Also, the material system used here is the same as in previous chapters. Namely, the thickness of each one of the eight layers of FerroA6 LTCC is $97 \mu \mathrm{m}$, while the relative permittivity is 5.7 .

\subsection{Design Specifications and Circuit Schematic}

The balun operates by introducing a 180 degree phase difference between the output ports (2 and 4 ) when power is applied to port 1. Meanwhile, port 3 remains isolated. A similar design methodology used for the 90 degree hybrid (Ch. 5) is pursued here where each transmission line is replaced with a lumped element equivalent. Three 90 degree pi networks are inserted between ports 1-2, 2-3 and 3-4, while a 270 degree (or -90 degree) highpass tee network is placed between ports 1-4. The lumped equivalent model of the balun is shown in Fig. 6.3 alongside the corresponding design equations $(6.1$ to 6.4$)[2]$.

$$
\begin{gathered}
L 1=\frac{\sqrt{2} Z_{0} \sin \theta}{\omega} \\
L 2=\frac{-\sqrt{2} Z_{0}}{\omega \sin \theta} \\
C 1=\frac{1}{\sqrt{2} Z_{0} \omega} \sqrt{\frac{1-\cos \theta}{1+\cos \theta}} \\
C 2=\frac{1}{\sqrt{2} Z_{0} \omega} \sqrt{\frac{1+\cos \theta}{1-\cos \theta}}
\end{gathered}
$$

The lumped element values are obtained by substituting $Z_{0}$ with $50 \Omega$ and $\omega$ with 


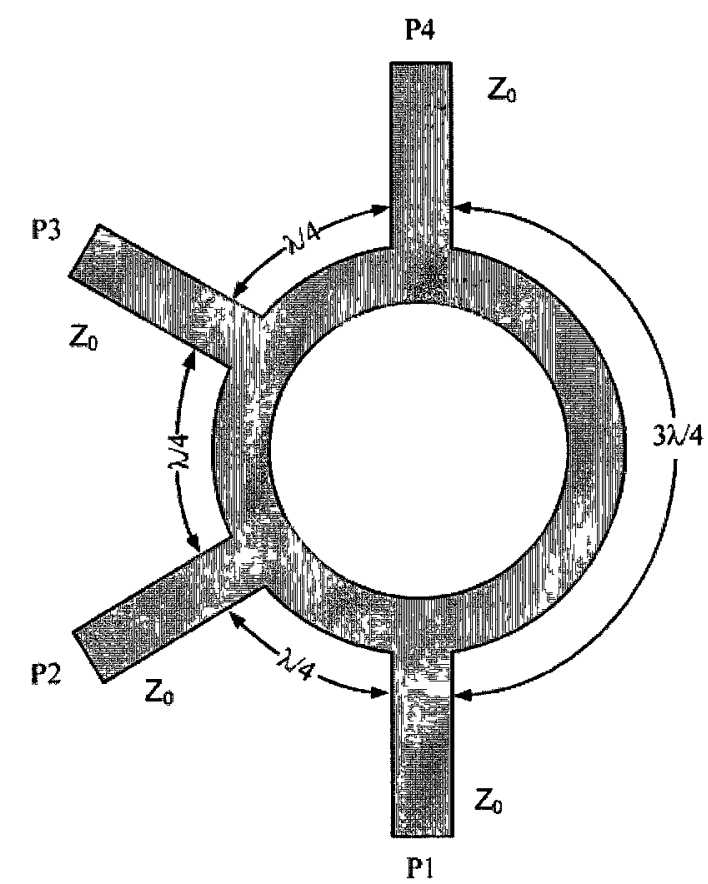

Figure 6 2. Classical distributed rat-race coupler layout.

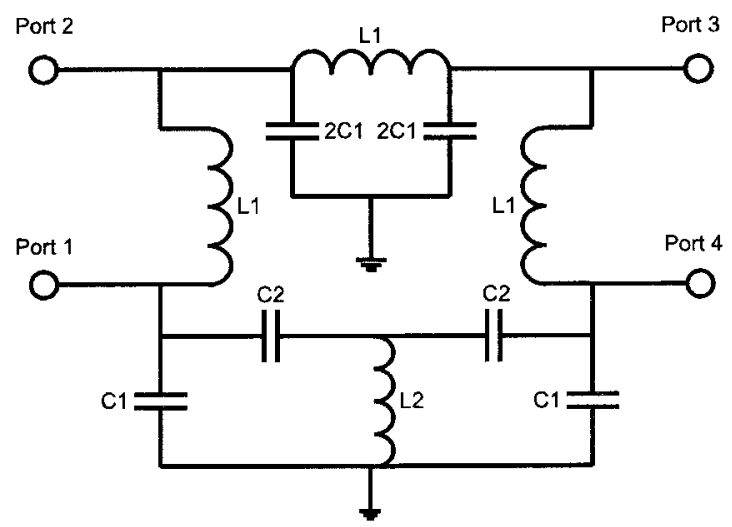

Figure 63 : Symmetric lumped element equivalent model for rat-race coupler. 
$2 \pi \times 1.55 \times 10^{9}$ in all equations, while, $\theta$ is substituted with 90 degrees in equations 6.1 and 6.3. In equations 6.2 and $6.4, \theta$ is replaced with -90 degrees. The balun is designed to have a centre frequency of $1.55 \mathrm{GHz}$ and $10 \mathrm{~dB}$ bandwidth of over $100 \mathrm{MHz}$, to account for fabrication tolerances. Insertion losses are to be kept as low as possible and an isolation of better than $20 \mathrm{~dB}$ is desired. Performing the calculations yields $7.25 \mathrm{nH}$ for both $L 1$ and $L 2$ and $1.45 \mathrm{pF}$ for both $C 1$ and $C 2$.

\subsection{Component Library Creation and Simulated Performance}

Before the complete balun structure could be assembled, each inductor and capacitor was simulated individually to provide the correct theoretical values. Capacitors $C 1$ and $C 2$ were implemented as 7 layer parallel plate designs, while the square shaped spiral inductors have $3^{1 / 4}$ turns each and also utilize 7 layers. As will be shown, the tuning (in simulation) of the balun to obtain optimal performance proved challenging. In fact, it was necessary to size the inductors independently of each other.

\subsubsection{Inductor Design}

A model of the inductor and how it was tuned is shown in Fig. 6.4. A rectangular helical structure is employed so that the tuning method used in Chapter 5 could also be employed here.

The theoretical calculations indicate that $L 1$ and $L 2$ should have the same inductance. However, in practice it was discovered that the balun could not be optimized without giving these inductors different values. The strategy used was to assign three 


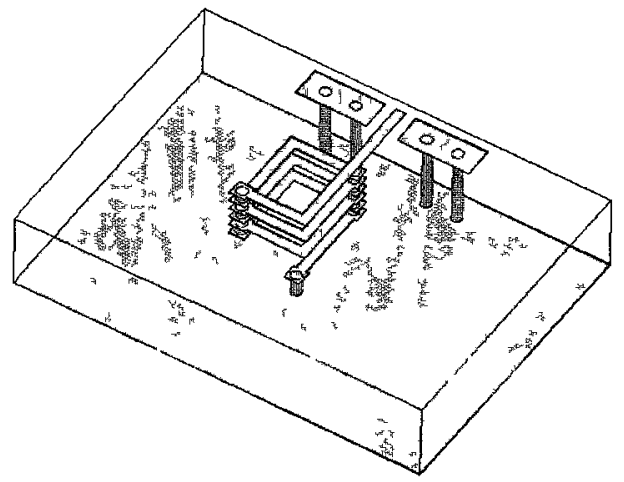

(a)

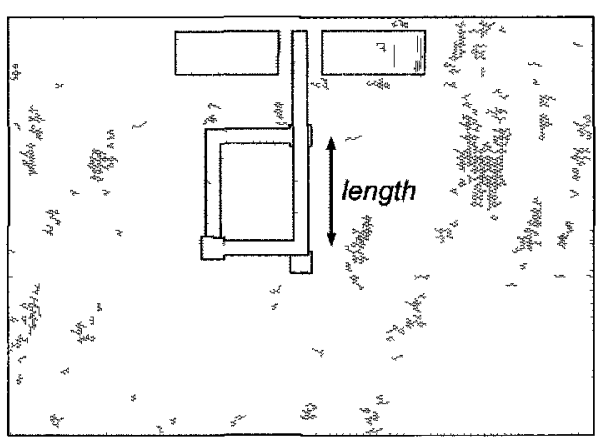

(b)

Figure 6.4: Multilayer spiral inductor structures: (a) three-dimensional model; and (b) length adjustment.

distinct design variables for the lengths of $L 2, L 1$ between ports 2-3, and the $L 1$ pair between ports 1-2 and 3-4 To remove any ambiguity, $L 1$ between ports 2-3 will be referred to as $L 3$.

The same inductor structure is used to synthesize the three required inductances but with different lengths for each. First, a parametric simulation was run that varied the length of the inductor (as shown in Fig. 6.4(b)) to obtain the basic inductance of $7.25 \mathrm{nH}$. Subsequently, this inductor was copied and placed in a way to implement the balun along with capacitors $C 1$ and $C 2$. The balun layout will be revealed in Section 63 along with a description of the methodology used to yield inductances of $89 \mathrm{nH}, 77 \mathrm{nH}$, and $9.2 \mathrm{nH}$ for $L 1, L 2$, and $L 3$, respectively The simulated Q-factor for these inductors varies between 55 and 65 . Corresponding plots of these quantities are displayed in Fig 6.5 


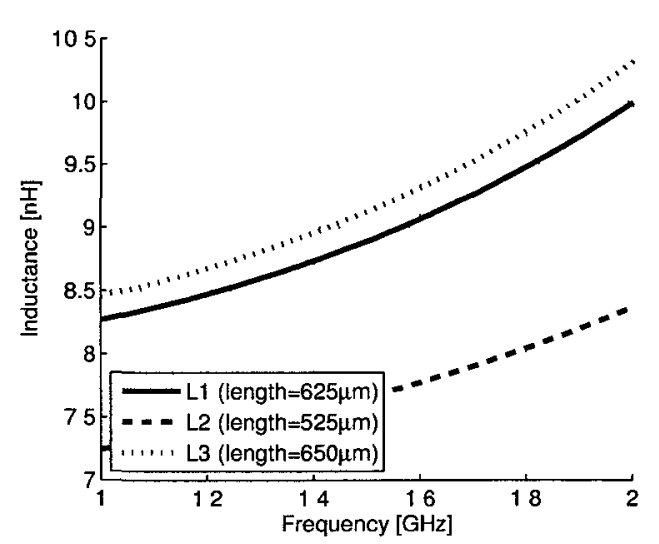

(a)

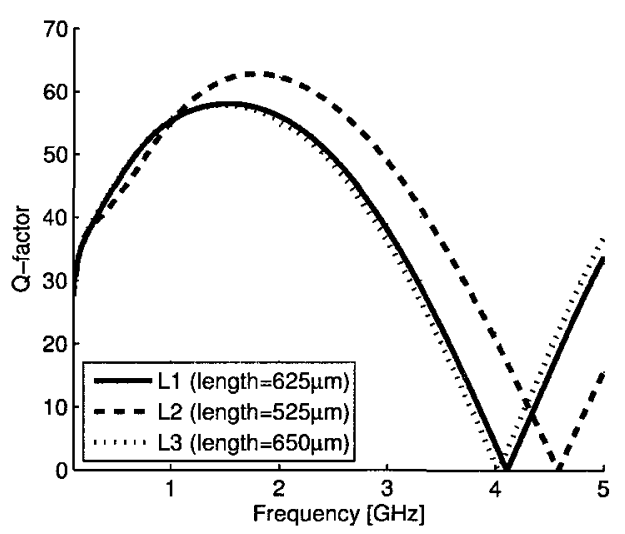

(b)

Figure 6.5: Simulated inductor performance for balun: (a) inductances and; (b) Qfactors.

\subsubsection{Capacitor Design}

The theoretical calculations indicate that both $C 1$ and $C 2$ should have the same capacitance. Indeed, this was maintained while optimizing the balun's performance. However, the larger capacitors whose values are $2 C 1$ were sized independently and did not maintain the theoretical ratio of $2: 1$. The capacitor model is not unlike that used for the 90-degree coupler save for the fact that here all plates are of equal size. The optimized capacitances and Q-factors are displayed in Fig. 6.6.

At $3.3 \mathrm{pF}$ for $C 1$ and $C_{2}$ and $4.2 \mathrm{pF}$ for $2 C 1$, the optimized capacitances are notably larger than theory suggests but this trend is consistent with what was experienced during the design of the 90-degree hybrid. 


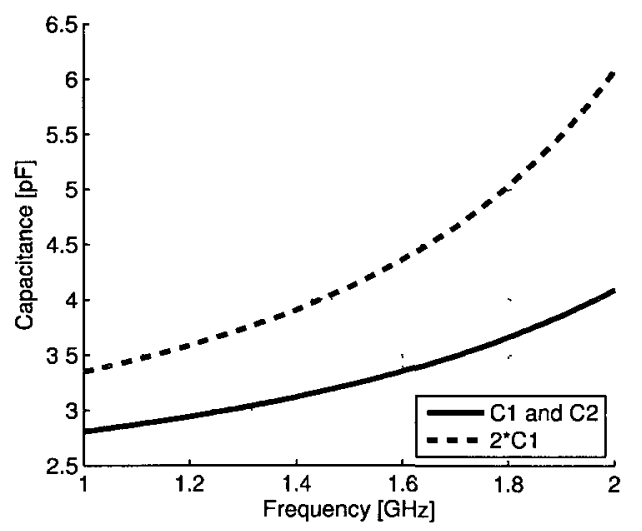

(a)

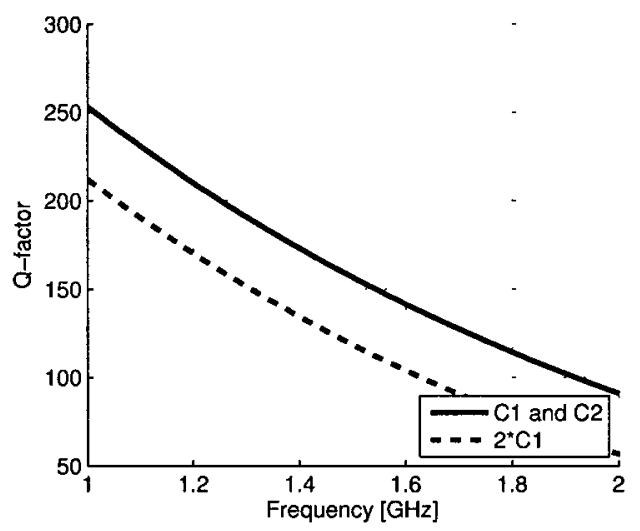

(b)

Figure 6.6: Simulated capacitor performance for balun: (a) capacitances; and (b) Q-factors.

\subsection{Balun Simulated Performance}

A three-dimensional rendering of the balun, showing embedded metal layers, alongside a top view of the structure is displayed in Fig. 6.7. In this figure, individual capacitors and inductors are labeled so that a comparison with the schematic representation can be made. The balun occupies only $4.4 \times 4.2 \times 0.679 \mathrm{~mm}^{3}$ of standard low permittivity LTCC substrate (7 layers). Miniaturization had a strong influence in the design of the balun. As can be seen in Fig. 6.7(a), the structure is very dense. The novel layout and packaging of the balun allows for an extreme degree of miniaturization that is unmatched in the literature. The smallest dimensions permitted by the foundry for both line width and separation were needed to obtain such a compact structure. In general, the topology of the model follows that of the schematic closely. However, a key reason for the model's compactness is that inductor L2 is folded inside and resides at the center of the balun structure (unlike in the schematic representation). In an effort to minimize resistive losses in the network, the number of vias used was 


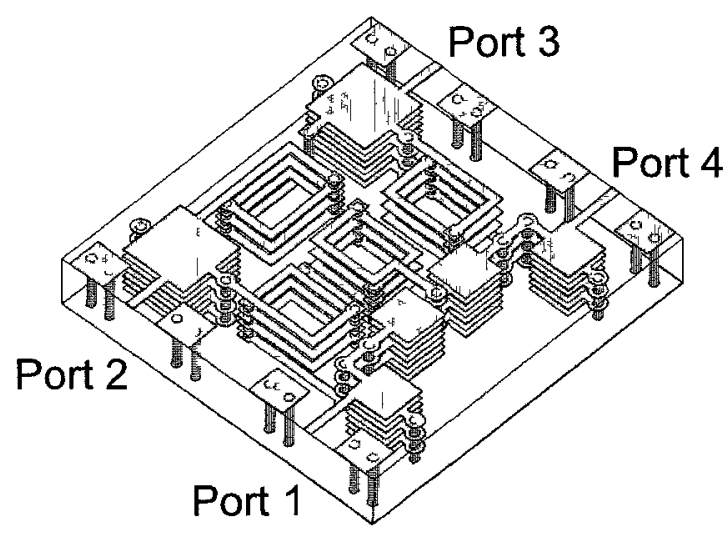

(a)

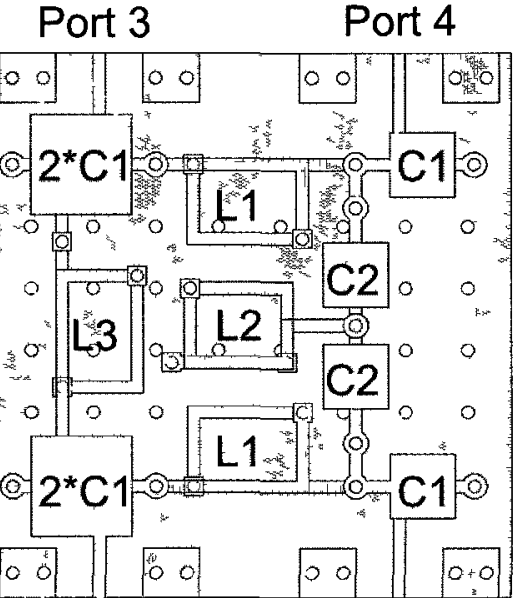

Port 2

(b)

Figure 6.7: Balun structure: (a) three dimensional view; and (b) top view.

reduced by combining as many nodes as possible.

Not surprisingly, the initial simulation of the complete balun revealed that it did not meet the required specificatıons. Specifically, the center frequency was too high and the amplitude balance of the output ports was more than $3 \mathrm{~dB}$ off. These problems can largely be attributed to mutual coupling between inductors. In order to effectively compensate for this phenomenon, the effect of each component on the balun's performance needs to be determined. As mentioned earlier, this is why independent design variables were assigned to the sizes of the inductors and capacitors. Through the perturbation of one component value at a time the following behaviors were observed for the balun structure:

1. $C 1$ and $C 2$ have a strong effect on the center frequency Increasing both reduces the balun's center frequency and vice-versa.

2. Changing L2 has a strong influence on output phase without greatly affecting 
amplitude balance.

3. Increasing the inductance between ports 2 and 3 reduces $S_{41}$.

4. Increasing the inductance between ports 1-2 and 3-4 increases $S_{41}$.

With this knowledge, it is now necessary to develop a methodology to tune the balun. Complicating matters is the fact that all variables in this design are interdependent to some degree. Nonetheless, the methodology that is found to be most effective is as follows: first, the center frequency of the balun was optimized by increasing capacitors $C 1$ and $C 2$; second, the amplitude balance was improved by adjusting the $L 1$ inductors; finally, L2 was increased slightly to achieve the correct phase shift. The final dimensions of each component were optimized in 17 iterations of manual tuning. Table 6.1 shows the initial and final values.

Table 6.1: Balun Optimization Results

\begin{tabular}{|c|c|c|c|c|c|}
\hline & $C 1[\mu \mathrm{m}]$ & $C 2[\mu \mathrm{m}]$ & $L 1[\mu \mathrm{m}]$ & $L 2[\mu \mathrm{m}]$ & $L 3[\mu \mathrm{m}]$ \\
\hline Initial & 630 & 450 & 500 & 500 & 500 \\
\hline Final & 810 & 525 & 625 & 525 & 650 \\
\hline
\end{tabular}

Plotting the magnetic fields within the substrate as presented in Fig. 6.8 is useful in gaining insight into the operation of the balun. From Fig. 6.8, at least two observations can be made: $i$ ) the magnetic fields of the inductors are tightly coupled, and $i$ ) port 3 is effectively isolated. The first observation confirms the earlier assertion that parasitic effects are a significant factor in the initial performance of the balun, while the second observation is confirmed by the S-parameter (specifically $S_{31}$ ) results presented next. 


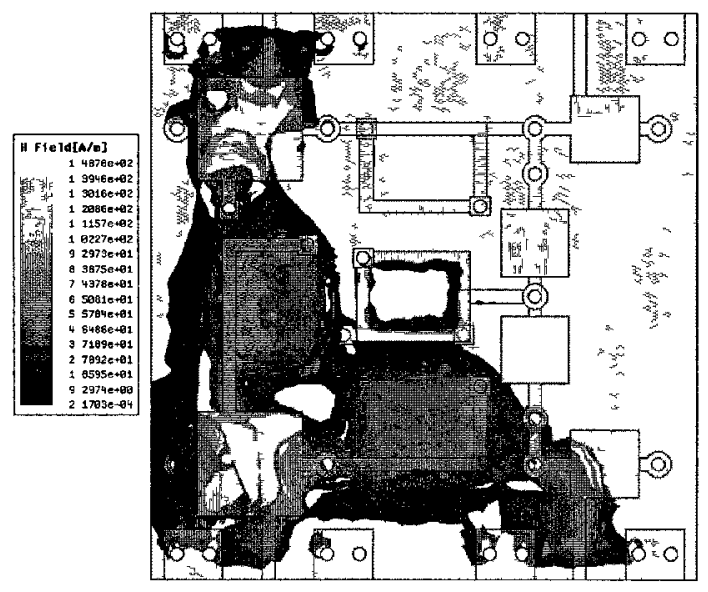

Figure 6.8: Magnetic fields within balun structure.

The complete simulated results for the optimized balun are displayed in Fig. 6.9. At the center frequency of $1.55 \mathrm{GHz}$, the insertion losses of ports 2 and 4 are equal to $3.4 \mathrm{~dB}$. The amplitude balance is very stable and does not exceed $0.7 \mathrm{~dB}$ from 1.4 to $1.8 \mathrm{GHz}$. The phase difference is only 0.5 degrees below the ideal 180 degrees. The return losses at ports 1 and $4\left(S_{11}, S_{44}\right)$ are below $10 \mathrm{~dB}$ from 1.3 to $1.7 \mathrm{GHz}$, while at port 2 the impedance bandwidth $\left(S_{22}\right)$ ranges from 1.1 to $1.7 \mathrm{GHz}$. At port 3 , the isolation $\left(S_{31}\right)$ is $26 \mathrm{~dB}$. This performance meets the design specifications.

\subsection{Balun Characterization}

A micrograph of the fabricated balun is shown in Fig. 6.10. The procedure used to measure the balun was identical to that used for the 90-degree hybrid coupler of Chapter 5. That is, a pair of G-S-G probes with a pitch of $500 \mu \mathrm{m}$ were employed to obtain 2-port S-parameter data (both magnitude and phase). The remaining two ports were loaded with $50 \Omega$ chip resistors. To obtain the full set of S-parameter data, 


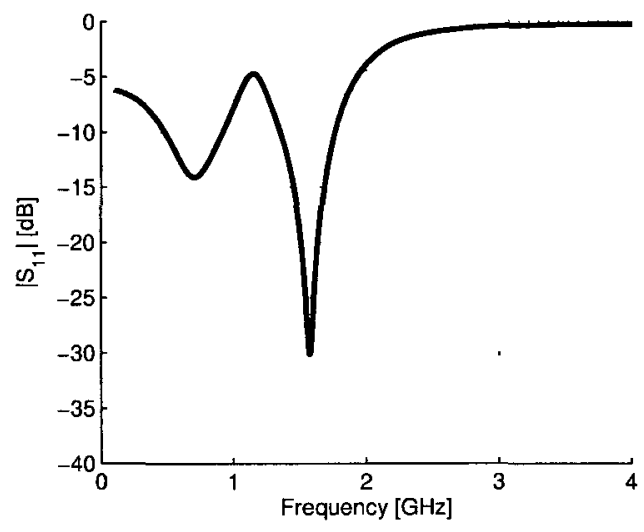

(a)

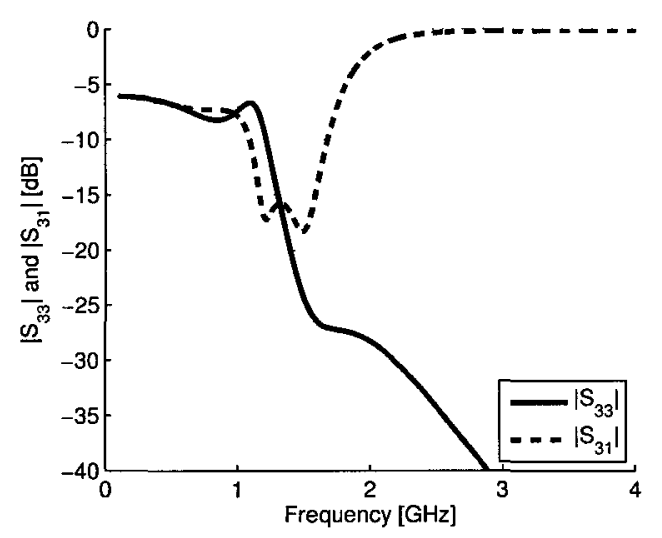

(c)

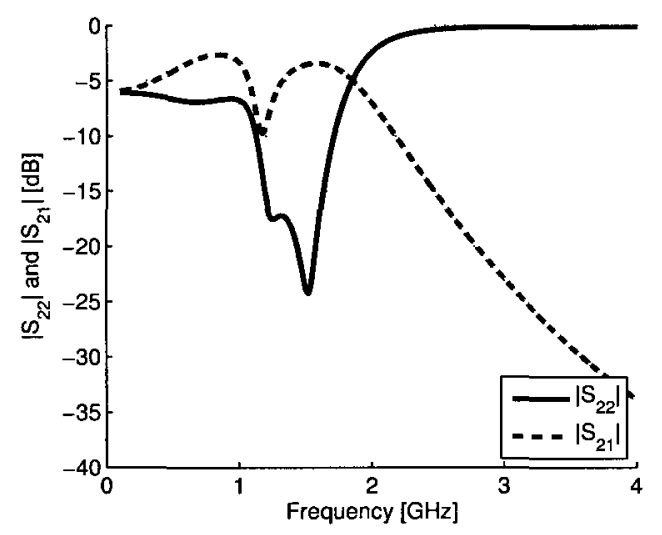

(b)

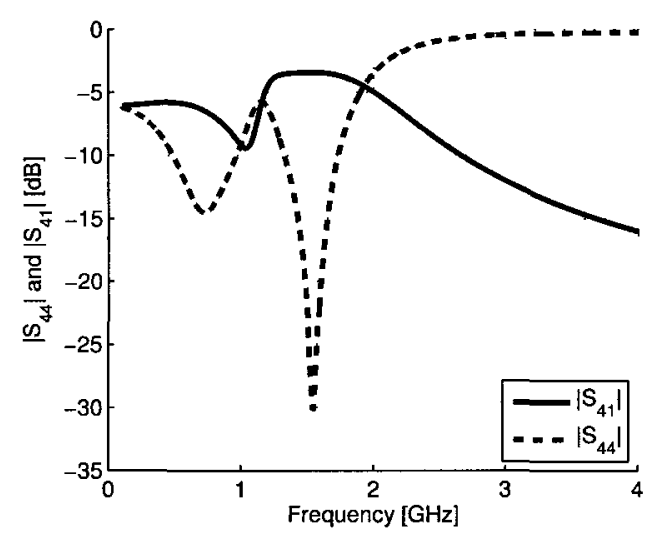

(d)

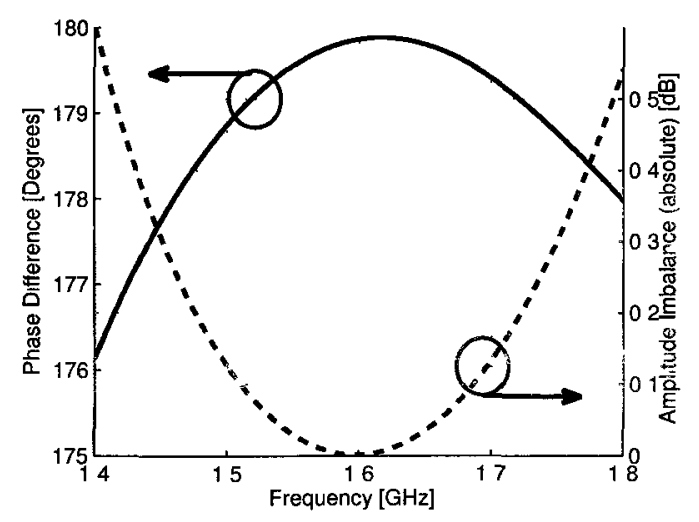

(e)

Figure 6.9: Simulated balun performance: (a) $S_{11}$; (b) $S_{22}$ and $S_{21}$; (c) $S_{33}$ and $S_{31}$; (d) $S_{44}$ and $S_{41}$; and (e) combined $\Delta \theta$ and amplitude imbalance. 


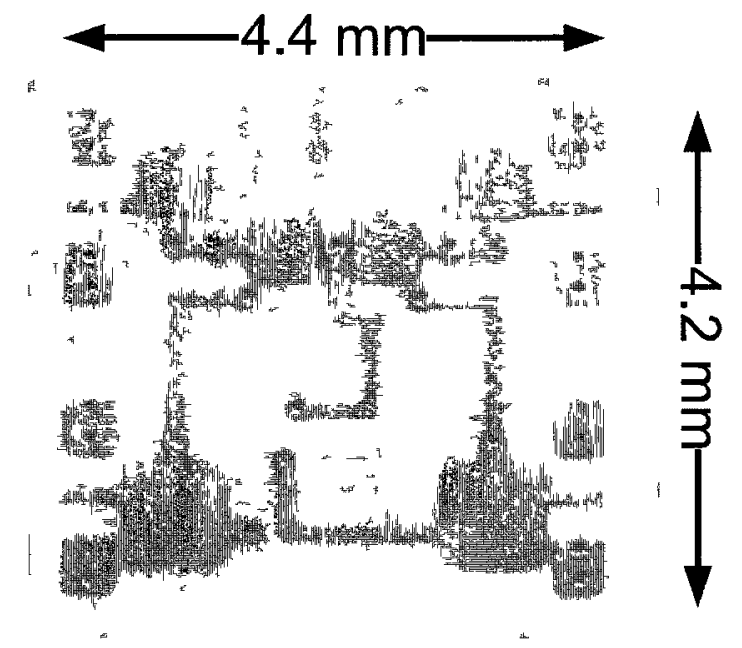

Figure 6 10: Micrograph of fabricated LTCC balun.

three measurement configurations were required and are outlined in Table 6.2. The specific S-parameters that can be obtained from each configuration are also listed in Table 6.2.

Table 6.2 Balun Measurement Configurations

\begin{tabular}{|c|c|c|c|c|c|}
\hline Configuration & Port 1 & Port 2 & Port 3 & Port 4 & S-Parameters \\
\hline 1 & Probe & Load & Load & Probe & $S_{\mathbf{1 1}}, S_{41}, S_{\mathbf{4 4}}$ \\
\hline 2 & Probe & Probe & Load & Load & $S_{21}, S_{22}$ \\
\hline 3 & Probe & Load & Probe & Probe & $S_{31}, S_{33}$ \\
\hline
\end{tabular}

The amplitude imbalance is calculated by subtracting the magnitudes of $S_{41}$ and $S_{21}$, whlle the phase difference is obtained by subtracting their phases A different copy of the balun was used for each measurement configuration This was done to prevent component swapping, which can cause metallızation damage and ultimately effect the accuracy of the results While, SOLT calibration was performed to the probe tips, the effect of the pads and loads was not deembeded A comparison of the 
simulated and measured results is shown in Fig. 6.11 with reasonable agreement in all cases.

The measurements indicate impedance bandwidths at all ports of almost $500 \mathrm{MHz}$ and more than sufficient for this application. Insertion losses at the output ports are less than $0.67 \mathrm{~dB}$ above the theoretical minimum. The phase difference of the outputs is 179.25 degrees, while the amplitude imbalance is below 1.2 $\mathrm{dB}$. A detailed summary and comparison of results is presented in Table 6.3. For parameters such as phase and amplitude balance, their variations are quoted based on the operating range of the balun, which is from $1.525 \mathrm{GHz}$ to $1.575 \mathrm{GHz}$.

Table 6.3: Balun Measurement Summary

\begin{tabular}{|c|c|c|}
\hline Item & Simulated & Measured \\
\hline Center Frequency & $1.57 \mathrm{GHz}$ & $1.55 \mathrm{GHz}$ \\
\hline $10 \mathrm{~dB}$ Return Loss BW at Port 1 & $410 \mathrm{MHz}$ & $560 \mathrm{MHz}$ \\
\hline$\left|S_{21}\right|$ & $-3.4 \mathrm{~dB}$ & $-3.5 \mathrm{~dB}$ \\
\hline$\left|S_{41}\right|$ & $-3.4 \mathrm{~dB}$ & $-3.67 \mathrm{~dB}$ \\
\hline Phase Difference & $179.5[$ degrees] & $179.25[$ degrees] \\
\hline$\left|S_{21}\right|$ Variation $[ \pm 50 \mathrm{MHz}]$ & $0.1 \mathrm{~dB}$ & $0.02 \mathrm{~dB}$ \\
\hline$\left|S_{41}\right|$ Variation $[ \pm 50 \mathrm{MHz}]$ & $0.01 \mathrm{~dB}$ & $0.02 \mathrm{~dB}$ \\
\hline Phase Balance $[ \pm 50 \mathrm{MHz}](\max )$ & $1[$ degrees] & $0.25[$ degrees] \\
\hline Amp. Imbalance $[ \pm 50 \mathrm{MHz}](\max )$ & $0.12 \mathrm{~dB}$ & $1.2 \mathrm{~dB}$ \\
\hline
\end{tabular}

The overall performance of the balun is very good. The poorest performance parameter is the amplitude imbalance. As displayed in Fig. 6.11(e), it is below $0.4 \mathrm{~dB}$ from $1.4 \mathrm{GHz}$ to $1.8 \mathrm{GHz}$ but rises significantly below this band. Although still within an acceptable range, the imbalance will limit the operating bandwidth of the 


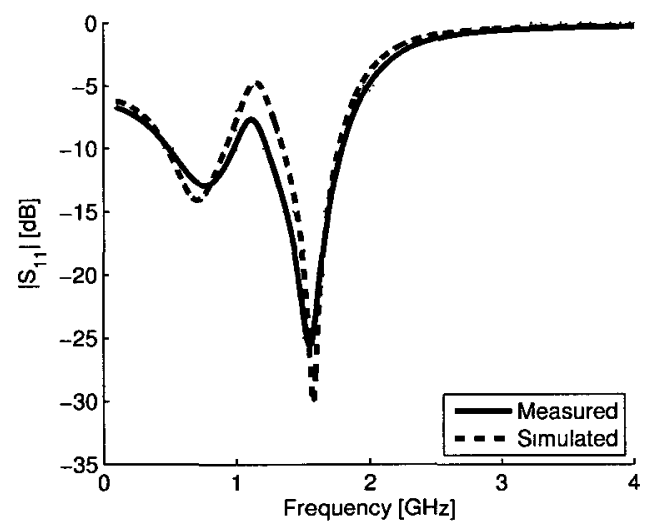

(a)

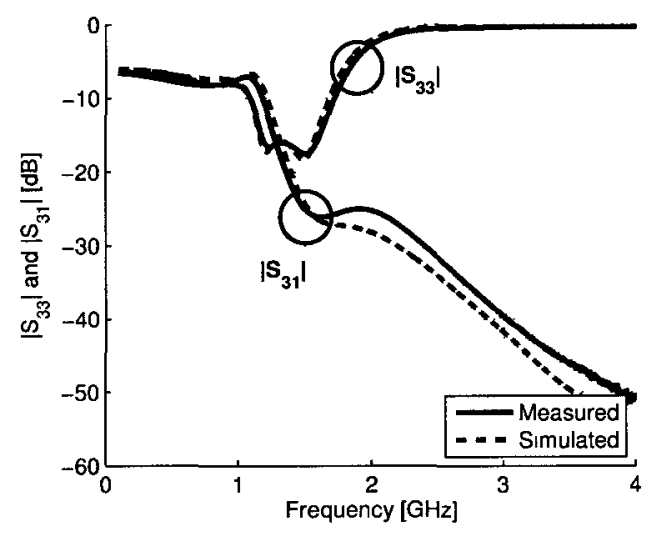

(c)

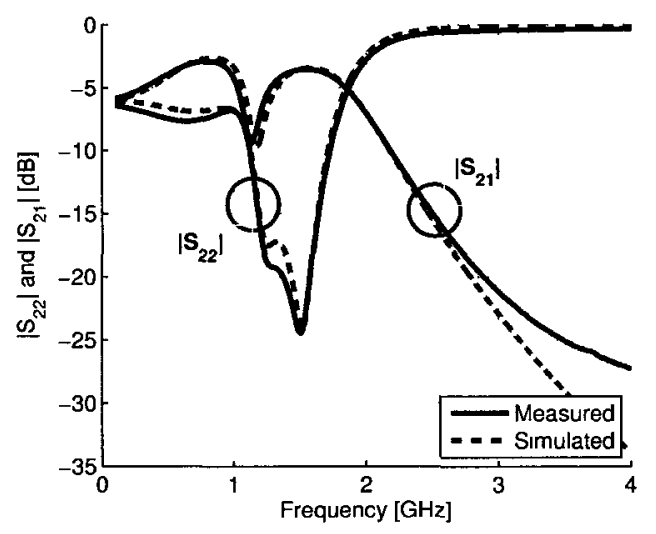

(b)

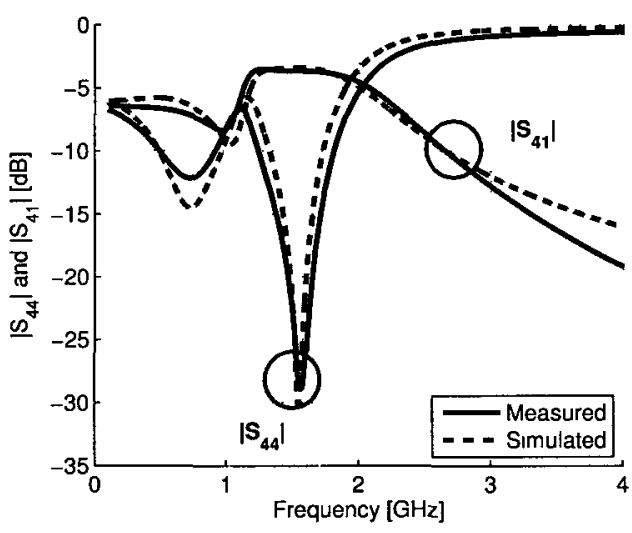

(d)

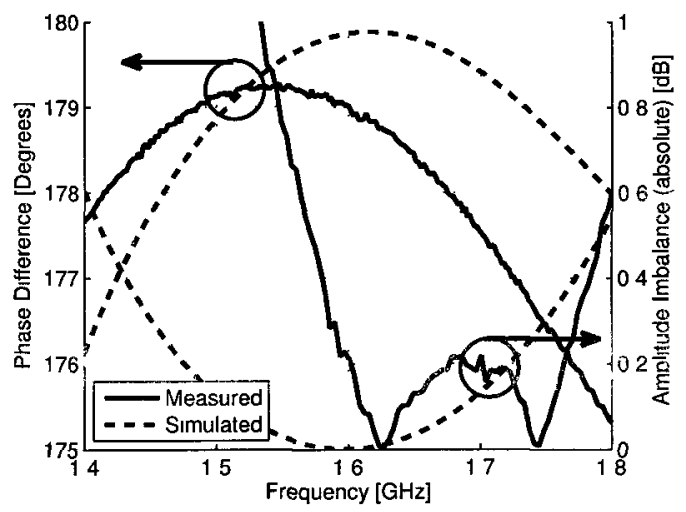

(e)

Figure 6.11: Meesured balun performance: (a) $S_{11}$; (b) $S_{22}$ and $S_{21}$; (c) $S_{33}$ and $S_{31}$; (d) $S_{44}$ and $S_{11}$, and (e) combined $\Delta \theta$ and amplitude imbalance. 
balun more than any other performance parameter. To determine the reason for this discrepancy, $L 1, L 2$, and $L 3$ were measured individually and their results presented in Fig. 6.12. For completeness, capacitor 2C1 was also measured and its results are plotted in Fig. 6.13.

These measurements show that the inductors have a tolerance of $+10 \%$, while the capacitor displays a tolerance of only $-5 \%$. These numbers and the Q-factor tolerances are listed in Table 6.4 .

Table 6.4: Measurement Summary - Balun Components

\begin{tabular}{|c|c|c|c|c|}
\hline Item at $\mathbf{1 5 5 0} \mathbf{M H z}$ & $\boldsymbol{L 1}$ & $\boldsymbol{L 2}$ & $\boldsymbol{L} \boldsymbol{2}$ & $\mathbf{2 C 1}$ \\
\hline Simulated Capacitance or Inductance & $8.9 \mathrm{nH}$ & $7.7 \mathrm{nH}$ & $9.2 \mathrm{nH}$ & $4.2 \mathrm{pF}$ \\
\hline Measured Capacitance or Inductance & $9.8 \mathrm{nH}$ & $8.4 \mathrm{nH}$ & $10.1 \mathrm{nH}$ & $4 \mathrm{pF}$ \\
\hline Observed Tolerance & $+10 \%$ & $+9 \%$ & $+10 \%$ & $-5 \%$ \\
\hline \hline Simulated Q-factor & 58 & 62 & 58 & 111 \\
\hline Measured Q-factor & 45 & 45 & 45 & 87 \\
\hline Observed Tolerance & $-29 \%$ & $-38 \%$ & $-29 \%$ & $-27 \%$ \\
\hline
\end{tabular}

In Section 6.3 observations were made about the balun's behavior when a single component value was changed. This aided in the optimization of the balun design but it can also be used here to help understand the cause of the amplitude imbalance. It was observed that $L 1$ and $L 3$ have opposite effects on the amplitude imbalance and since their tolerances are identical they are not the likely cause. This leaves $L \mathscr{Z}$ and $2 C 1$ as better candidates so their tolerances were incorporated into a post-simulation with the results displayed in Fig. 6.14. 


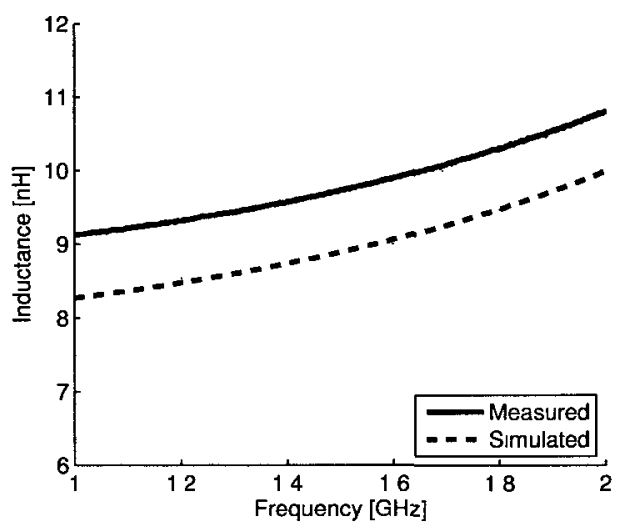

(a)

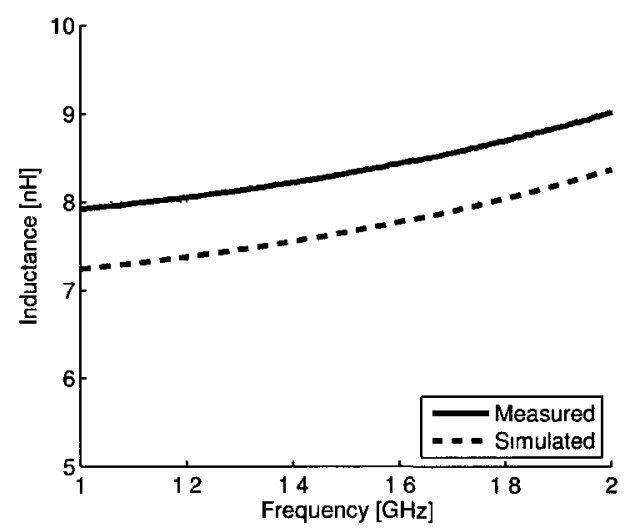

(c)

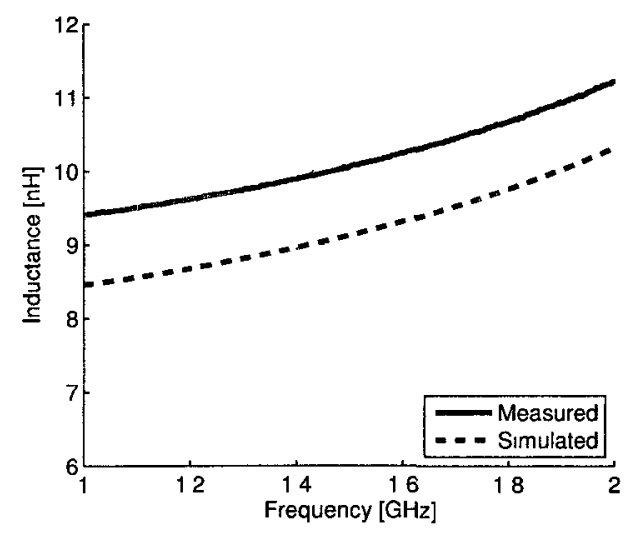

(e)

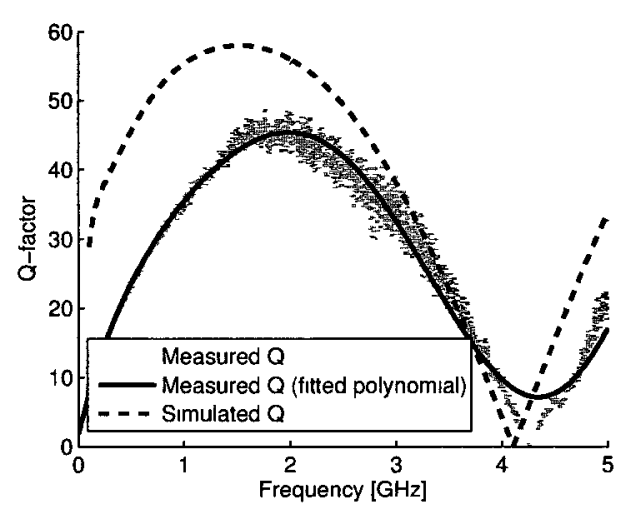

(b)

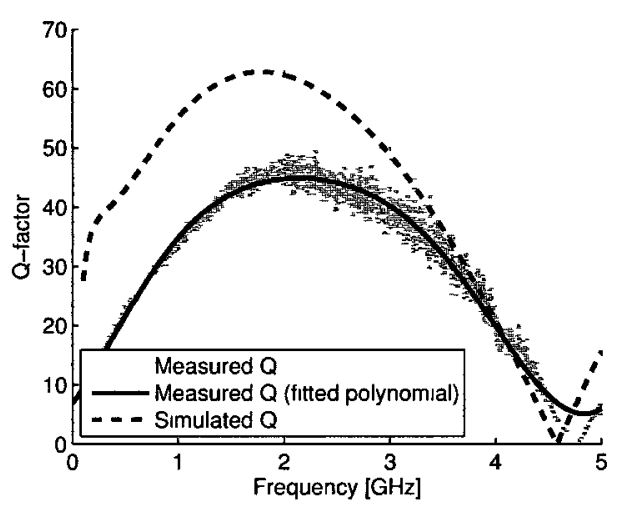

(d)

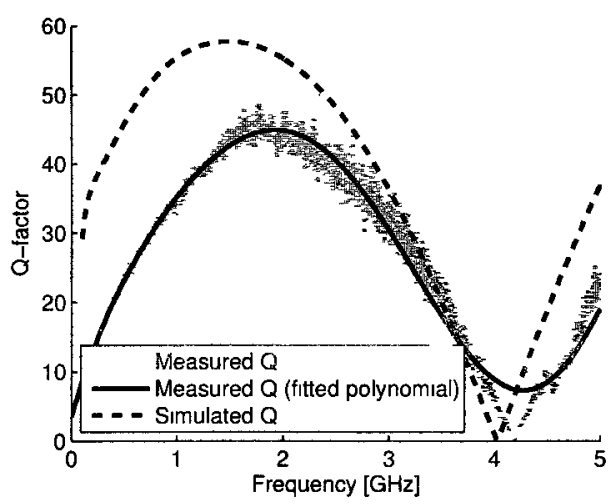

(f)

Figure 6.12: Measured inductance and Q-factor for: (a)-(b) L1; (c)-(d) L2; and (e)-(f) $L 3$. 


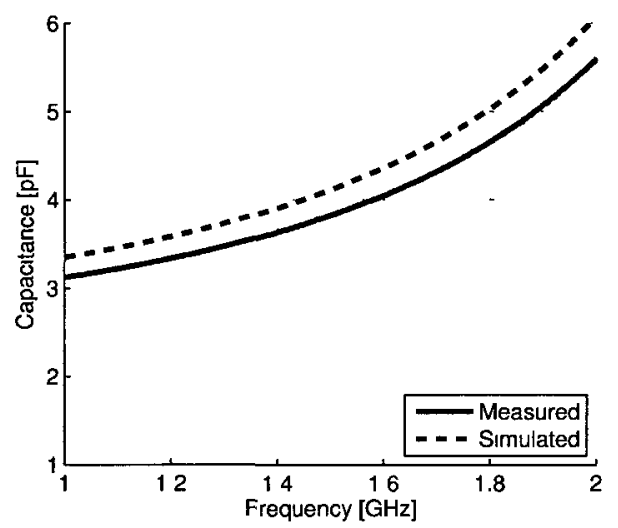

(a)

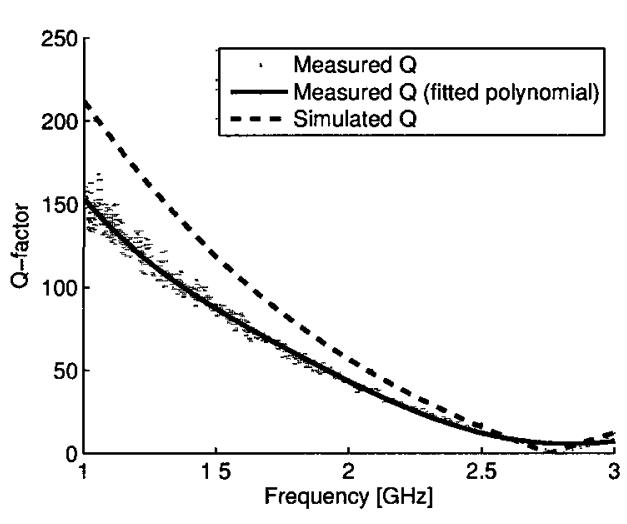

(b)

Figure 6.13: Measured 2C1 performance: (a) inductance and; (b) Q-factor.

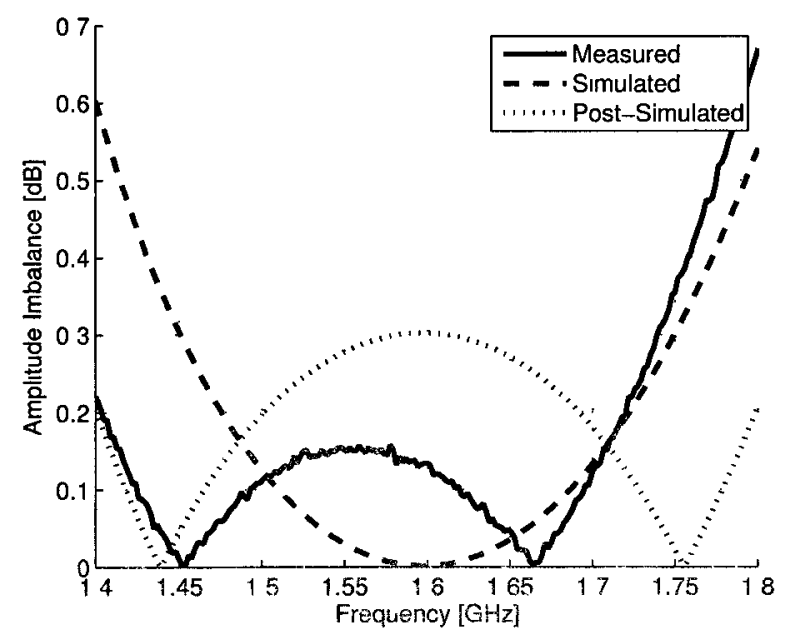

Figure 6.14: A comparison of the measured, simulated, and post-simulated results for the balun. 
The plot of amplitude imbalance shows a better match between the postsimulation and measurements than the original simulation. This confirms the supposition that tolerances of $L \mathscr{2}$ and $2 C 1$ are responsible for the added amplitude imbalance. Therefore, in future designs extra care should be taken to reduce them on these components in particular.

\subsection{Summary and Contribution}

This chapter has presented a new high performance balun with an extreme degree of miniaturization that represents the partial fulfilment of the fourth thesis contribution. A methodology was presented that allowed for the successful optimization of the design with relatively few iterations needed. Measured results show insertion losses within $0.67 \mathrm{~dB}$ of the theoretical maximum, an amplitude imbalance of less than $1.2 \mathrm{~dB}$ and a phase difference of 179.25 degrees. The performance of this balun make it a suitable choice for use in sensitive communication equipment as a surface mount component or embedded within an SoP environment. Furthermore, its small size makes it possible to place near the feed point of an antenna to create a compact balun-antenna combination.

To prove its high performance, a comparison with literature was attempted. Unfortunately, as was the case with the 90 -degree hybrid coupler of Chapter 5, comparable work could not be found. Instead commercial components from TDK, Murata, and Johnson Technology were used for comparison in Table 6.5. The parameter variations are determined from the quoted operating bandwidths of their respective components. The operating bandwidth of the balun here is from $1.525 \mathrm{GHz}$ to $1.575 \mathrm{GHz}$.

In comparison with these components from leading manufacturers the performance 
Table 6.5: Comparison with Commercial Components

\begin{tabular}{|l|c|c|c|c|}
\hline Item & This Work & TDK & Murata & Johnson \\
\hline Model No. & N/A & HHM1526 & LDB211G8110C-001 & 1600BL15B050 \\
\hline $\begin{array}{l}\text { Center Freq. } \\
\text { [MHz] }\end{array}$ & 1550 & 1850 & 1810 & 1600 \\
\hline $\begin{array}{l}\text { Bandwidth - } \\
\text { Input [MHz] }\end{array}$ & 560 & 280 & 200 & 200 \\
\hline $\begin{array}{l}\text { I. Loss [dB] } \\
\text { (max) }\end{array}$ & 0.67 & 0.8 & 0.9 & 1 \\
\hline $\begin{array}{l}\text { Phase Bal- } \\
\text { ance [degrees] } \\
\text { (max) }\end{array}$ & 0.25 & 9 & N/A & 2 \\
\hline $\begin{array}{l}\text { Amplitude } \\
\text { Imbalance } \\
\text { [dB] (max) }\end{array}$ & 1.2 & 1 & N/A & \\
\hline
\end{tabular}


of the balun presented here distinguishes itself as the best in all categories. To the author's knowledge, no other balun demonstrates such good phase balance, insertion loss, and even amplitude imbalance. 


\section{Chapter 7}

\section{Antenna Element Design}

In accordance with the fourth thesis objective, this chapter presents a novel antenna element whose topology is based on the study of Chapter 2. The antenna design is revealed along with simulations and measurements showing return loss and radiation patterns. The desired frequency of operation is $1.55 \mathrm{GHz}$. It's position in the receiver chain is displayed in Fig. 7.1.

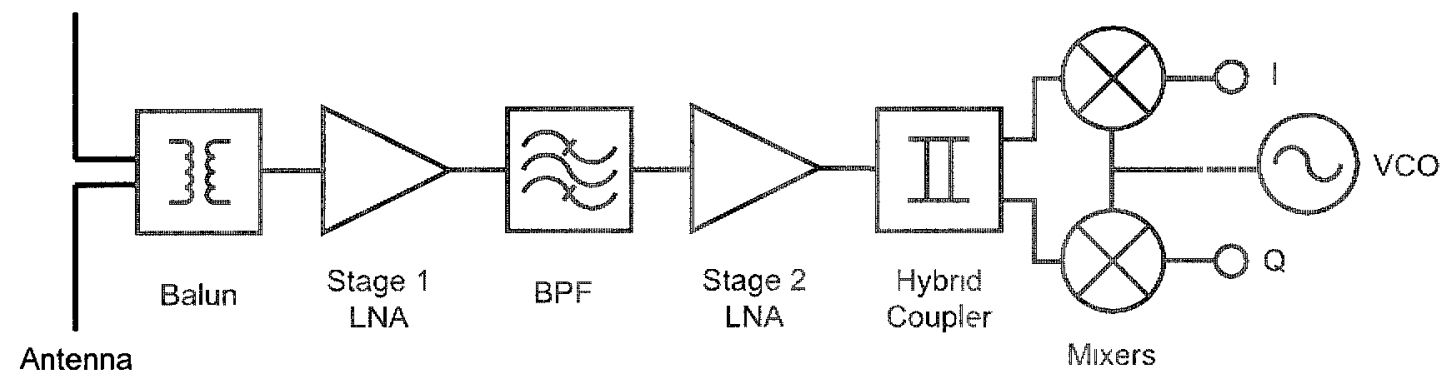

Figure 7.1: Antenna as part of the complete receiver chain.

The antenna's differential port will be converted to a singlc-ended port with the use of the balun described in chapter 6 . The antenna will be designed with an ideal feed before the balun is introduced and the resulting antenna-balun combination is optimized. This co-design methodology improves the likelihood of satisfying all 
requirements.

\subsection{Antenna Concept and Initial Design}

The quality of GPS antennas has a strong influence on the overall performance of a receiver due to weak satellite signals. Many antenna topologies have been adapted for GPS applications. However, not every type of antenna element is suitable for SoP integration. Most importantly, the element should be planar to occupy as few substrate layers as possible. Also, it should lend itself to common miniaturization techniques such as meandering and folding to minimize its size.

A large body of work already exists on the subject of general antenna miniaturization techniques in the form of journal articles [33,101-103] and textbooks [58,104,105]. These techniques can be divided into two main categories: i) material and ii) reactive loading [106]. Material loading refers to the application of materials that have a high relative permittivity or permeability, while reactive loading increases the selfinductance and/or shunt capacitance of an antenna's structure. Reactive loading is often the preferred technique because it doesn't suffer from additional losses associated with material loading. Reactive loading can be achieved by way of geometrical transformations that alter the current distribution of standard antenna elements. This can be accomplished by introducing slots or notches that force surface currents to travel along a longer path than normal [107]. Lumped element loading can work in the same way to reduce the reactive component of an antenna's input impedance so that it becomes resonant at a lower frequency [108]. For dipoles in particular, geometrical transformations like bending, meandering, or some other sort of space maximization technique are common $[109,110]$. The degree of miniaturization achievable, however, 
is governed by fundamental limits $[106,111]$. An antenna's gain, bandwidth, and efficiency decrease as these limits are approached. The antenna design presented here uses a combination of material and reactive loading, with the latter component being dominant.

When discussing the relative size and performance of antennas it is important to specify what size ground plane is required, if any. This was already proven in Chapter 2 in reference to monopole and microstrip antennas. Moreover, studies by S.R. Best have shown that the size and location of a PIFA antenna's ground plane may have a significant impact on its performance as well $[50,112]$. By virtue of being housed in a small package, the antenna for this application should not be dependent on a ground plane. An omnidirectional radiation pattern is preferred, especially for a receiver whose orientation in space may be arbitrary. For simplicity, we have restricted ourselves to linearly polarized elements in this work.

Based on the study in Chapter 2, a dipole antenna is proposed for integration into the GPS SoP module. Since this is a resonant element, the total length of a basic dipole is approximately half of a wavelength in freespace $\left(\lambda_{0} / 2\right)$. At $1.55 \mathrm{GHz}$ this equates to $95 \mathrm{~mm}$. Given the stated dimensions of the SoP module $(32 \times 24 \mathrm{x}$ $2.5 \mathrm{~mm}^{3}$ ) yielding a perimeter of $112 \mathrm{~mm}$, such a long dipole would be expected to be accommodated by routing the dipole arms around the perimeter of the module. However, simulating this simple solution results in a self resonance at $1.85 \mathrm{GHz}$ and input impedance of $12+\mathrm{j} 347 \Omega$ at $1.55 \mathrm{GHz}$. Such a low resistance would make matching to $50 \Omega$ challenging. The input impedance can be increased by lengthening the dipole but due to the limited area available, miniaturization techniques are needed. Meandering of the dipole arms is a well known and effective method [113-117]. Fig. 7.2(a) presents the concept of meandering, while Fig. 7.2(b) shows its applications in 
this antenna design.

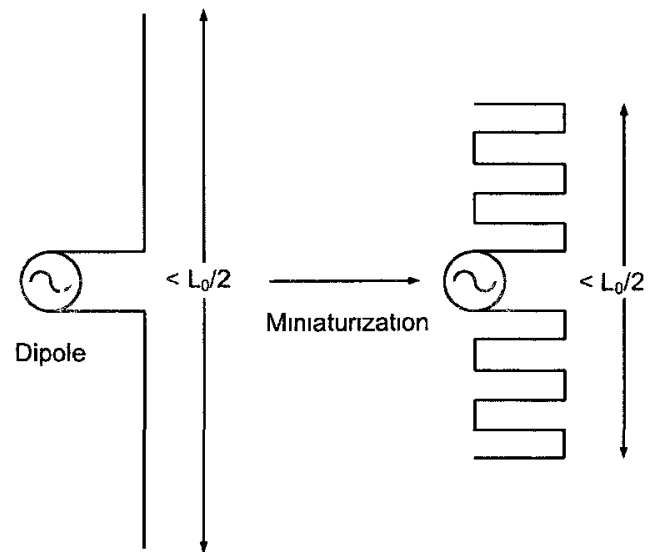

(a)

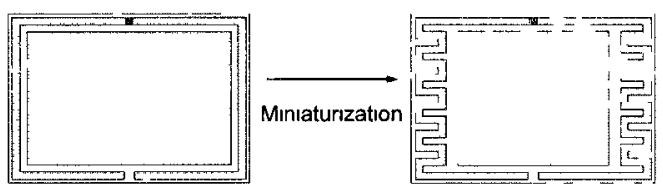

(b)

Figure 7.2: Miniaturization of a conventional dipole by the meandering of each arm: (a) concept; and (b) realization.

By meandering the dipole arms, a length of $0.8 \lambda$ or $160 \mathrm{~mm}$ is achieved and the self resonant frequency lowers to $1.7 \mathrm{GHz}$. The input resistance at $1.55 \mathrm{GHz}$ becomes $50 \Omega$ but due to the introduction of inductive loading, the input reactance jumps to j768 $\Omega$ [118]. A comparison of the simulated input resistances for both the $0.5 \lambda$ and $0.8 \lambda$ length dipoles is shown in Fig. 7.3.

\subsection{Antenna Design and Optimization}

The actual proposed dipole layout is shown in Fig. 7.4. The antenna arms are placed around the perimeter of the module to maximize unobstructed space for the placement of receiver components. In simulation, an ideal excitation is used to feed the dipole with a differential signal at one edge of the module. It is known that this geometry will cause unwanted field cancelation due to the creation of parallel current lines with 


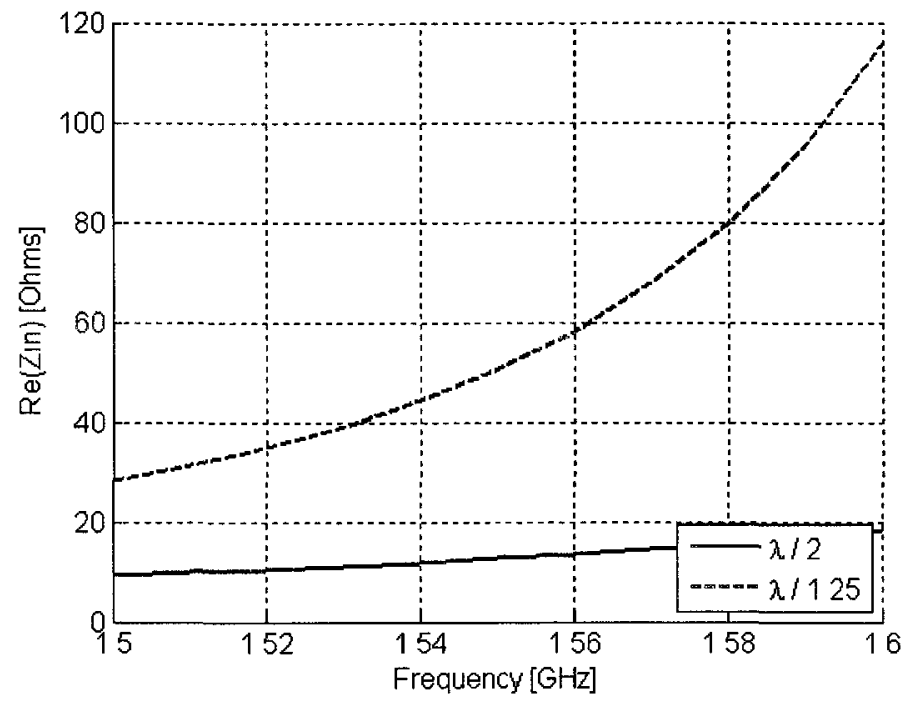

Figure 7.3: Comparison of input resistances for two different length dipoles.

opposite directions. Better radiation efficiency could be achieved with a centralized feed and the arms oriented in a "Z" pattern. However, this would critically limit the space for receiver components and therefore was not adopted. The location of the lossless lumped element impedance matching circuit is shown. Impedance matching is necessary to cancel out the large inductive component of the input impedance and will be described in the next section.

The material system used is the same as in [6] except the parameters have been updated based on more recent findings. The thickness of each layer of FerroA6 LTCC has been adjusted to $97 \mu \mathrm{m}$ (from $100 \mu \mathrm{m}$ ) and the substrate's relative permittivity has been reduced to 5.7 (previously 5.9) The loss tangent (0.002), the conductor (silver) and the number of layers (8) remains unchanged. However, the top and bottom metal layers are gold plated to reduce oxidation and surface roughness.

As discussed, the dipole is miniaturized by meandering each arm to occupy area 
as efficiently as possible. The dipole metallization is located on the bottom of the substrate along with a ground plane. It is important to note that the ground plane is not necessary for the operation of the dipole, rather it is there to be used by other receiver components (such as the $\mathrm{BPF}$, coupler, etc.). However, the distance from the ground plane to the antenna adds significant capacitıve loadıng. This distance, labeled as $d$ in Fig. 7.4 along with the arm width $(w)$, separation $(s)$, and meander length $(l)$, represent the three most important design variables.

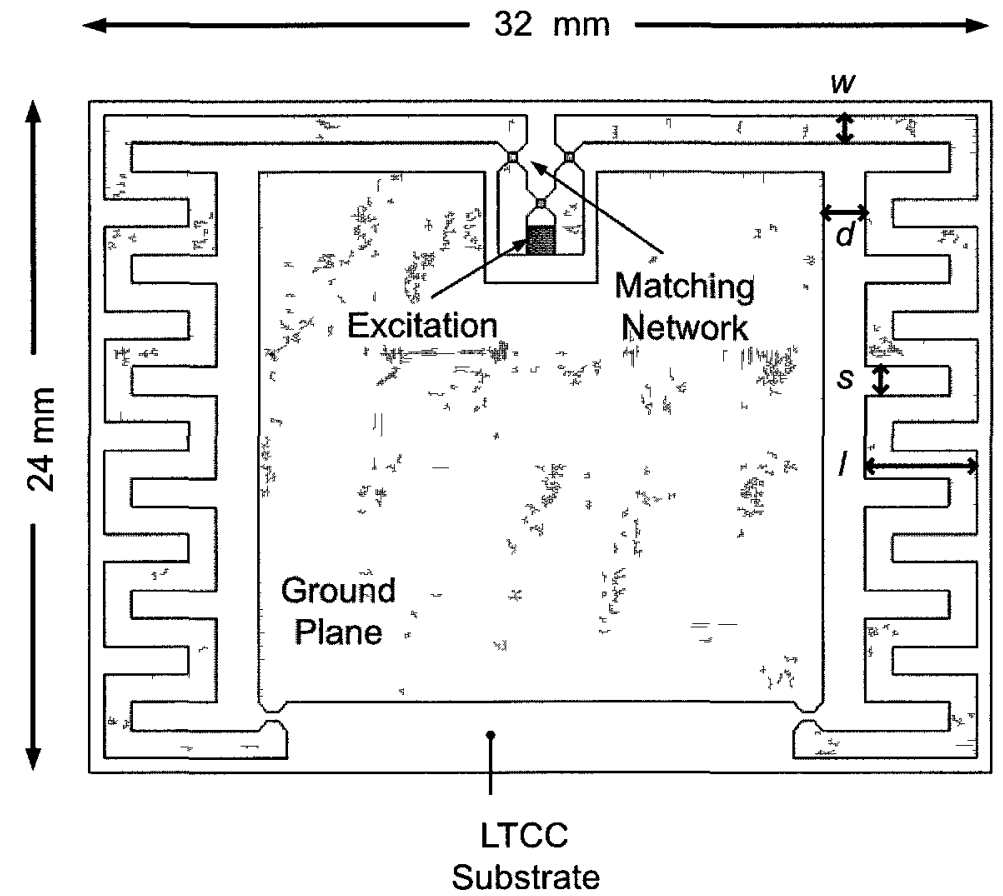

Figure 7.4: Proposed miniaturized dipole layout.

The first step in the design process involves determining a value for $w$. Choosing a small $w$ will increase the ohmic losses in the dipole. On the other hand, a large $w$ will reduce the maximum antenna length achievable and space for circuit components. A 
width of $1.0 \mathrm{~mm}$ was deemed to be a good compromise. For $s$, the important consideration is capacitive loading that increases with decreasing $s$. This type of loading can lead to additional ohmic losses if the electric field strength between adjacent traces is high. Therefore, selecting a relatively large separation of $1.0 \mathrm{~mm}$ avoids this unwanted effect. Similarly, if $d<1.0 \mathrm{~mm}$ the losses will be elevated so setting $d=1.5 \mathrm{~mm}$ bypasses this problem as well. The variable $l$ is solely determined by the desired length of the dipole. Obviously, setting a large $l$ will result in almost no meandering of the arms. In this case, the minimum length of $3 \mathrm{~mm}$ was selected so that more meander sections could be accommodated. A summary of the final variable values is presented in Table 7.1.

Table 7.1: Final values for antenna design variables

\begin{tabular}{|c|c|}
\hline Variable & Optimized Value $[\mathrm{mm}]$ \\
\hline \hline$w$ & 1.0 \\
\hline$s$ & 1.0 \\
\hline$d$ & 3 \\
\hline$l$ & 1.5 \\
\hline
\end{tabular}

\subsection{Matching Network}

An ideal $\lambda / 2$ dipole has an input resistance of $73 \Omega$ along with a small positive reactance. In this case, a matching network may even be unnecessary if the length of the dipole can be changed freely to tune its impedance. In reality, most resonant antennas require a matching network to transform the complex driving point impedance to a purely real value, typically $50 \Omega$. Such is the case in this printed dipole design. 
The input impedance of the miniaturized dipole is $26+\mathrm{j} 278 \Omega$, which differs from the simplified miniaturized dipole discussed earlier that does not feature a matching network layout. This discrepancy is not too surprising since the feed area of a dipole is known to be very sensitive to geometrical changes, especially when the currents there are small [58]. Importantly, the resistance is high enough that a matching network with reasonable element values can still be created.

L-section matching is used to obtain the element values of the parallel inductor and series capacitors. Calculations based on the analytical equations from [23] are used to obtain element values. A high pass matching network, presented in Fig. 7.5(a), is chosen because the normalized load impedance lies outside of the $1+\mathrm{j} x$ circle on the Smith chart.

The network of Fig. 7.5(a) is suitable for matching a single-ended antenna, where $Z_{\text {ant_se }}$ represents the single-ended antenna impedance. In this work, the antenna has a differential input. Therefore, a modification to the network needs to be made by noting that a virtual ground exists in between the dipole arms. The equivalent matching network is shown in Fig. 7.5(b).

One arm of the dipole is excited by a $50 \Omega$ port referenced to ground, while the other is loaded with $50 \Omega$ to obtain a $Z_{\text {ant_se }}$ of $8.8+\mathrm{j} 128 \Omega$. Finding the values for the lumped components can be done using the Smith chart or with analytical equations. Either method will result in series capacitor and shunt inductor values of $0.918 \mathrm{pF}$ and $2.32 \mathrm{nH}$, respectively. Further tuning of these theoretical numbers in simulation yielded final values of $0.95 \mathrm{pF}$ and $3 \mathrm{nH}$. Surface mount 0402 components are used to realize the matching network as opposed to embedded elements due to the LTCC fabrication tolerances and the narrow bandwidth of the dipole. As shown in Fig. 7.4, the matching network is located near the feed point of the antenna and is comprised 


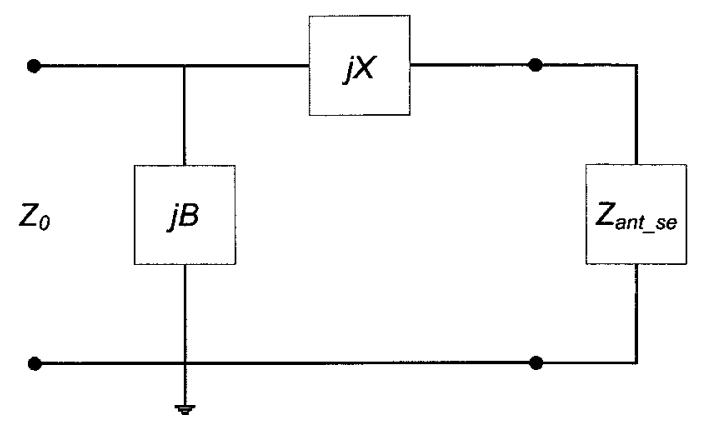

(a)

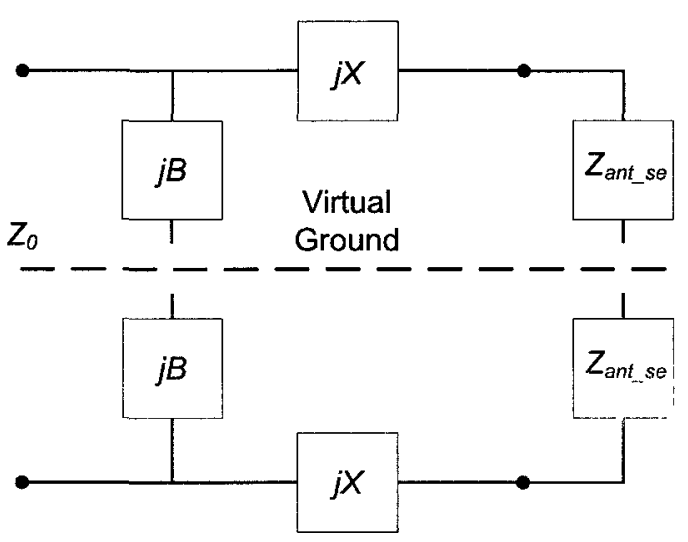

(b)

Figure 7.5: L-section matching network for: (a) single-ended; and (b) differential antennas.

of three pads. The end-loading pads are located, as expected, at the ends of the dipole arms and are also sized for 0402 passive components.

\subsection{Simulated Results of Final Design}

The preliminary antenna design of section 7.1 is now combined with the matching network of section 7.3 to produce a final impedance matched SoP antenna.

\subsubsection{Ideal Feed}

Given an ideal differential feed and lossless L, C matching elements, Fig. 7.6 shows that the matching network improves the return loss substantially. The $10 \mathrm{~dB}$ bandwidth is $20 \mathrm{MHz}$ or $1.27 \%$ and extends from $1.565 \mathrm{GHz}$ to $1.585 \mathrm{GHz}$.

For an ideal dipole, the radiation patterns are typically generated based on a coordinate system that has the z-axis oriented along the dipole arms. In this way, the $\theta=90$ cut (azimuth) displays a constant pattern, while the $\phi=0$ cut (elevation) 


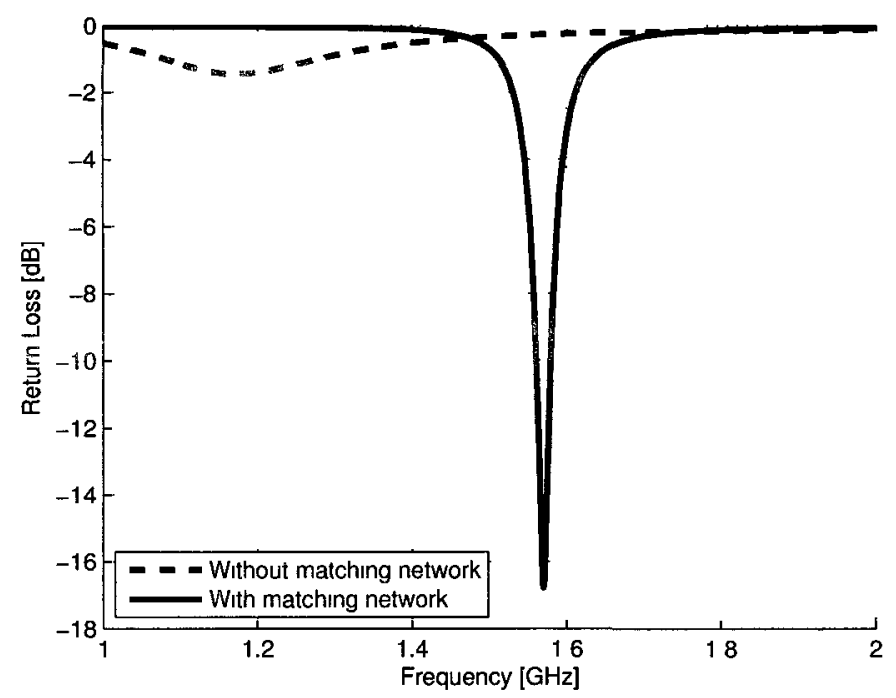

Figure 7.6: A comparison of the return loss for the SoP dipole with and without a matching network.

yields a directional pattern. The measurement setup used later, however, uses a different orientation that is adopted in simulations for consistency. The coordinate system employed here along with a 3-dimensional rendering of the the antenna pattern is shown in Fig. 7.7.

The azimuth cut is now defined as the Z-Y plane, while the elevation cut is in the Y-X plane. The dipole in this work differs from the ideal case in that its arms are bent at 90 degrees to almost create a square loop. However, a dipole pattern is expected to be generated since the highest intensity surface currents are located on the antenna sections that are parallel to the $\mathrm{x}$-axis.

Simulated principal plane cuts are presented in Fig. 7.8. They indicate a dipolelike radiation pattern with a peak gain of $0 \mathrm{dBi}$. While this result falls short of the theoretically expected gain of $1.7 \mathrm{dBi}$, it is nonetheless consistent with the fact that 


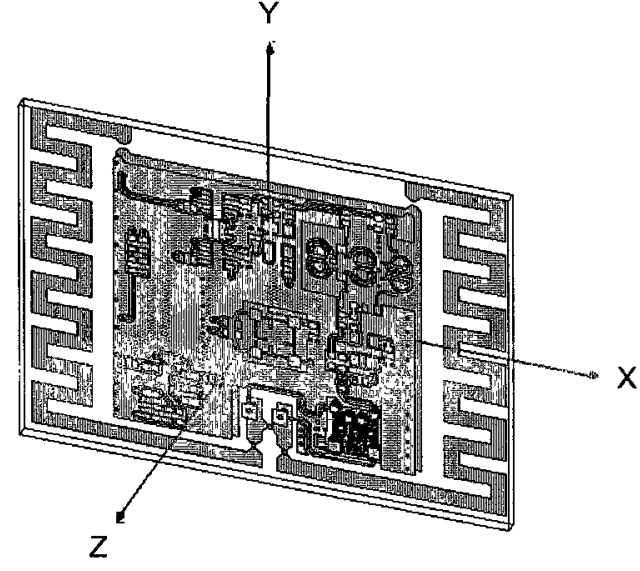

(a)

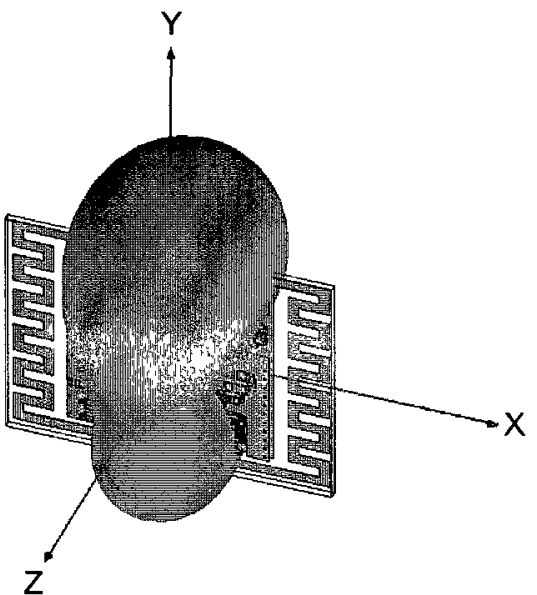

(b)

Figure 7.7: (a) Coordinate system used for radiation patterns; (b) Simulated 3dimensional result referenced to antenna model.

field cancelation (due to the bent dipole arms) and material losses contribute to lower gain. The simulated radiation efficiency of the dipole is $75 \%$, however, this result does not include the losses due to real matching elements. A better estimate of efficiency will be provided later.

\subsubsection{Balun Feed}

The 180-degree hybrid coupler described in Chapter 6 is used as a balun to feed the dipole. Although its amplitude and phase responses are very good, it's expected that the dipole will not perform as well as with the ideal feed. The balun is placed as close to the dipole terminals as possible to minimize line losses. Furthermore, Co-Planar Waveguide (CPW) lines are used, as opposed to microstrip, to reduce crosstalk and allow for a partial ground plane to be printed on the top metal layer of the module. Since the transitions from microstrip to CPW are all much smaller than 


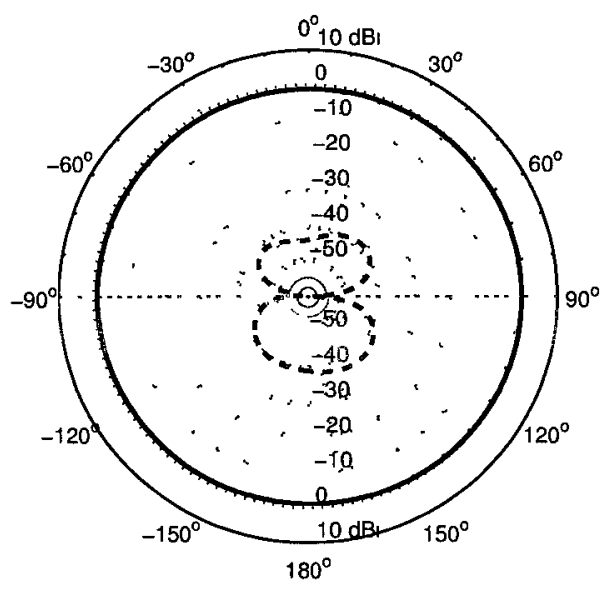

(a) Azimuth (Z-Y plane)

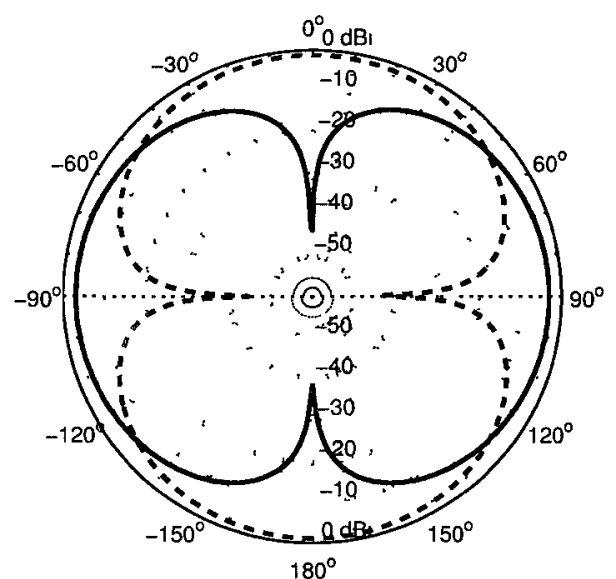

(b) Elevation (Y-X plane)

Figure 7.8: Simulated radiation patterns with ideal port excitation and lossless matching elements. Co-pol (solid line) and cross-pol (dashed line) results are shown.

a wavelength, special design considerations are not required. These features, along with the complete top metal layer for the module, are shown in Fig. 7.9(a) where the substrate is transparent and outlined only. It is important to note that the output of the balun is located on the top metal layer, while the antenna is located on the bottom metal layer. This necessitates two vias and sections of CPW lines, presented in Fig. 7.9(b), to connect the antenna terminals to the balun outputs.

Feeding the dipole with the balun introduces additional losses that can be seen in the radiation patterns of Fig. 7.10. A portion of these losses is attributed to the presence of metal traces and slots associated with the inclusion of the top metal layer of the module. Although the patterns of both principal planes retain the same shape, the peak gain drops to $-1.6 \mathrm{dBi}$ and cross-pol levels rise slightly. Furthermore, the efficiency drops by $12 \%$ to $63 \%$ compared to the ideal port case. 


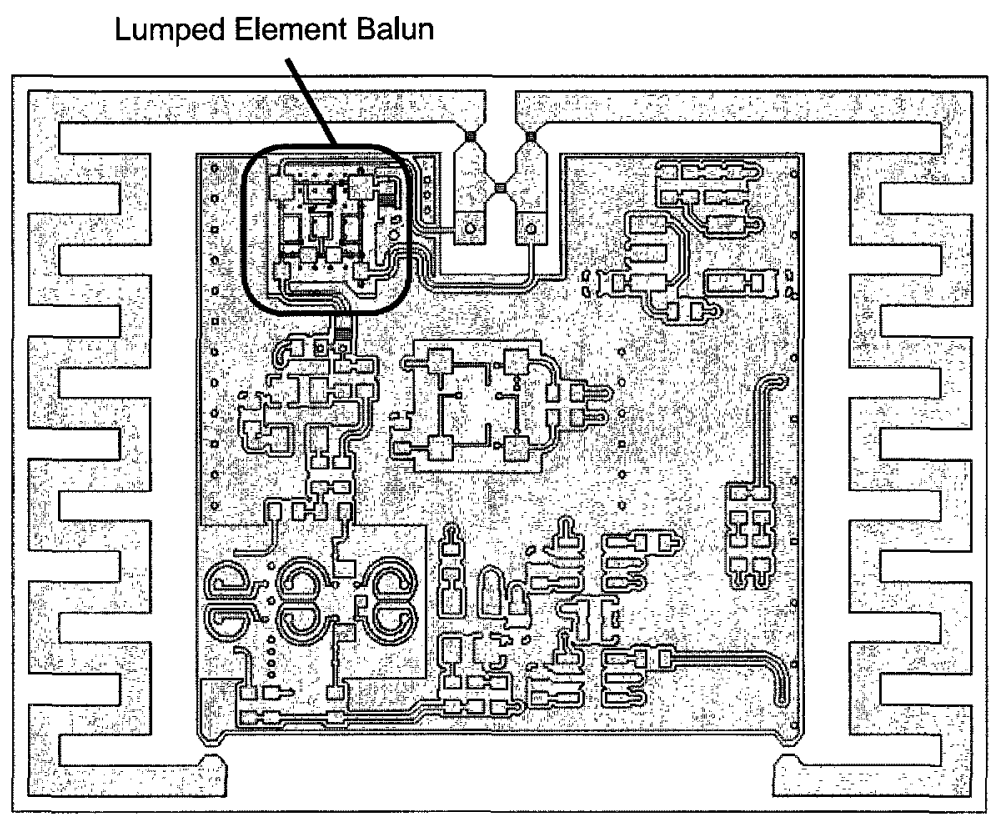

(a)

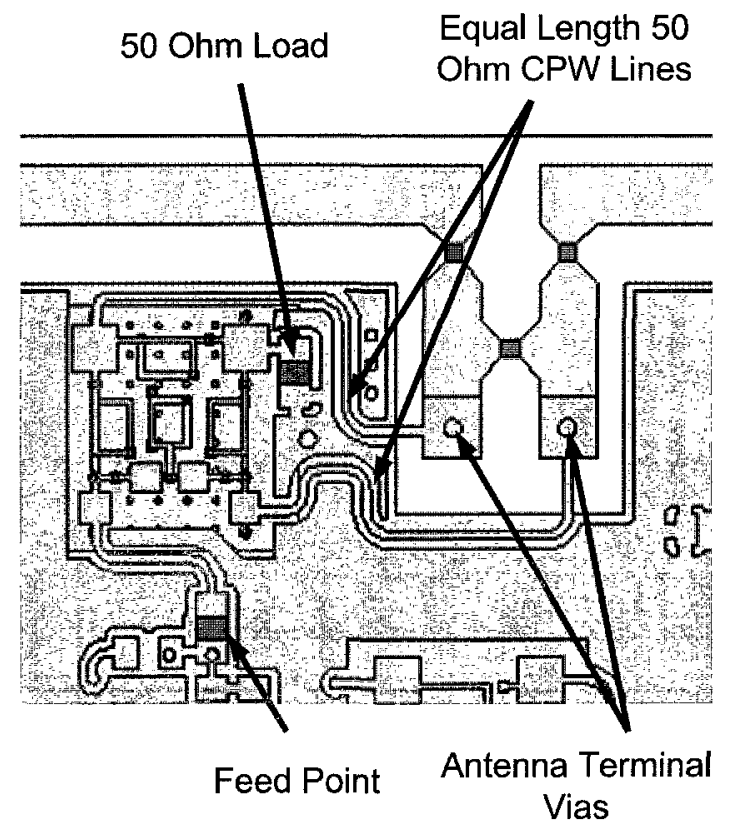

(b)

Figure 7.9: Location of (a) lumped element balun; and (b) detailed image of balun. 


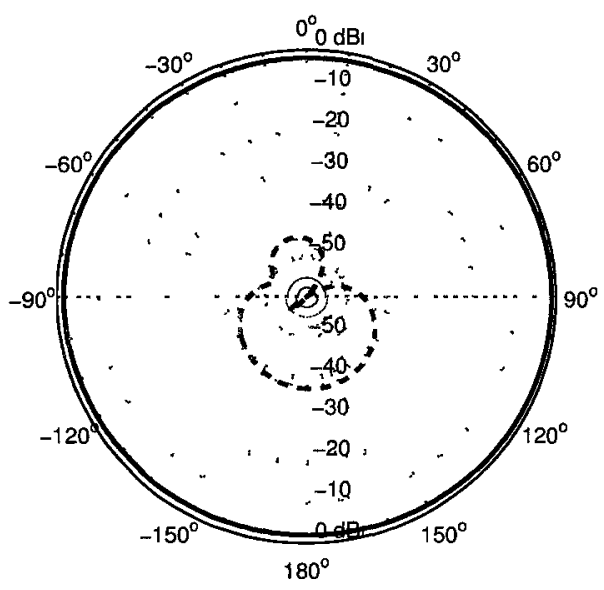

(a) Azimuth (Z-Y plane)

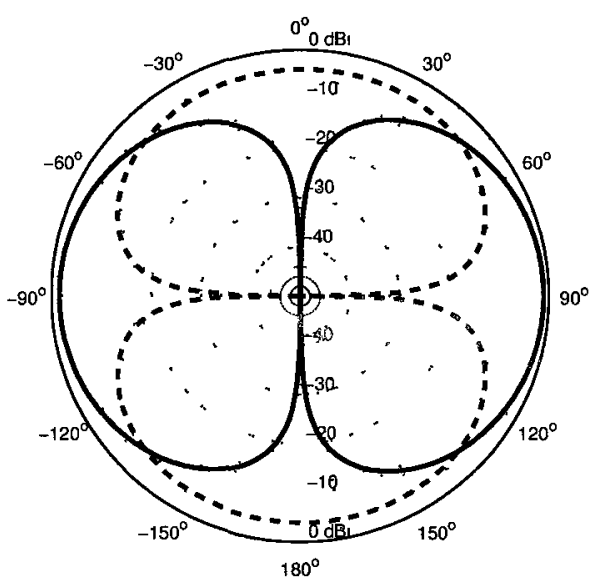

(b) Elevation (Y-X plane)

Figure 7.10: Simulated radiation patterns with balun feed. Co-pol (solid line) and cross-pol (dashed line) results.

Only minor de-tuning of the antenna is observed when the balun feed is used. As shown in Fig. 7.11, the center frequency shifts lower by $25 \mathrm{MHz}$. As will be discussed next, this can be compensated by reducing the value of the series capacitors of the matching network.

To summarize, Table 7.2 provides a list of differences in the performance of both dipole configurations discussed above.

\subsection{Measured Results}

The LTCC SoP module containing the antenna was fabricated at VTT Technical Institute in Oulu, Finland. In Fig. 8.10(a), the top layer of the SoP is visible along with the antenna terminals. The bottom side of the SoP module, which contains the 


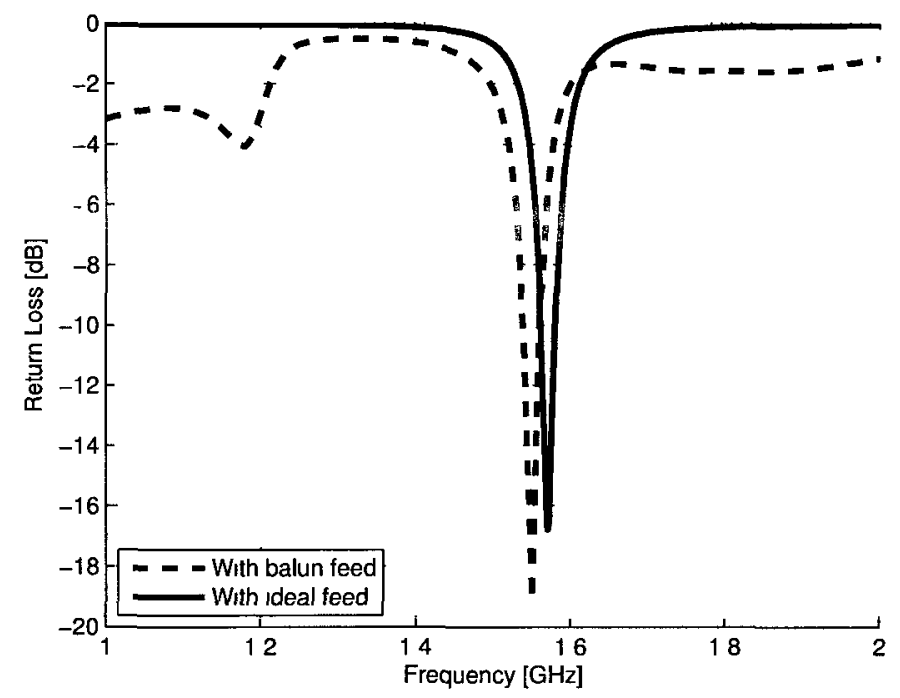

Figure 7.11: Performance difference for ideal and balun fed dipole.

Table 7.2: Differences in dipole performance when using an ideal port or embedded balun feed

\begin{tabular}{|c|c|c|}
\hline & Ideal Feed & Balun Feed \\
\hline Peak Gain $[\mathrm{dBi}]$ & 0 & -1.6 \\
\hline Efficiency $[\%]$ & 75 & 63 \\
\hline Center Frequency $[\mathrm{MHz}]$ & 1575 & 1550 \\
\hline Bandwidth $[\mathrm{MHz}]$ & 20 & 20 \\
\hline
\end{tabular}


antenna is presented in Fig. 8.10(b). Here, the pad locations for the input/output port, matching network and end-loading of the dipole are indicated. End-loading is a technique that may be used to increase the impedance bandwidth of an antenna or reduce its frequency of operation $[59,106]$.

A conventional SMA connector cannot be used as a port for the dipole because of the small size of the module. Instead, a miniature surface mountable coaxial connector is used, the location of which is shown in Fig. 7.13. This type of connector, called a "W.FL", is only $2 \mathrm{~mm} \times 2 \mathrm{~mm}$ in size and is also used for all other input/output ports on the module.

To effectively test the antenna and balun combination, the entire receiver was disconnected and the output redirected to a test port. High quality passives were chosen and attached with conductive epoxy to their respective pads on the package. The typical Q-factors for the SMT capacitors and inductor are 300 and 70, respectively. Although high, these finite Q-factors will reduce the gain and efficiency of the antenna. The input/ouput port is located on the bottom side of the package and is accessed by the aforementioned W.FL connector, which is connected to a miniature coaxial cable that finally is adapted to a standard SMA cable that travels to the measurement equipment. This setup is shown in Fig. 7.13.

Return loss was measured by an Agilent 8275 VNA in a Faraday cage that was SOLT calibrated up to the antenna's SMA adapter. Fig. 7.14 displays return loss curves for capacitor values ranging from $0.6 \mathrm{pF}$ to $0.9 \mathrm{pF}$.

As shown in Fig. 7.14, decreasing the capacitance has the converse effect on the antenna's resonance frequency. The nominal capacitance of $0.9 \mathrm{pF}$ achieves a good match but the frequency of $1.47 \mathrm{GHz}$ where it occurs is outside the desired bandwidth. The likeliest reason for this discrepancy is that all the embedded layers of the SoP 


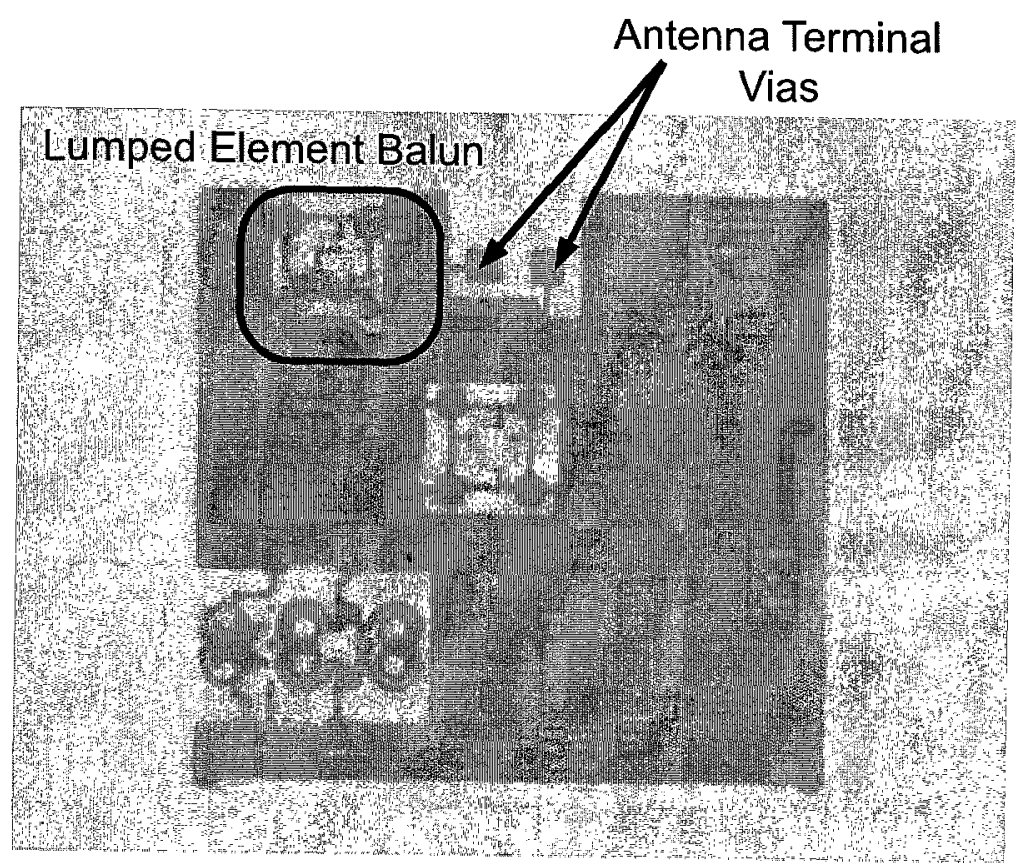

(a)

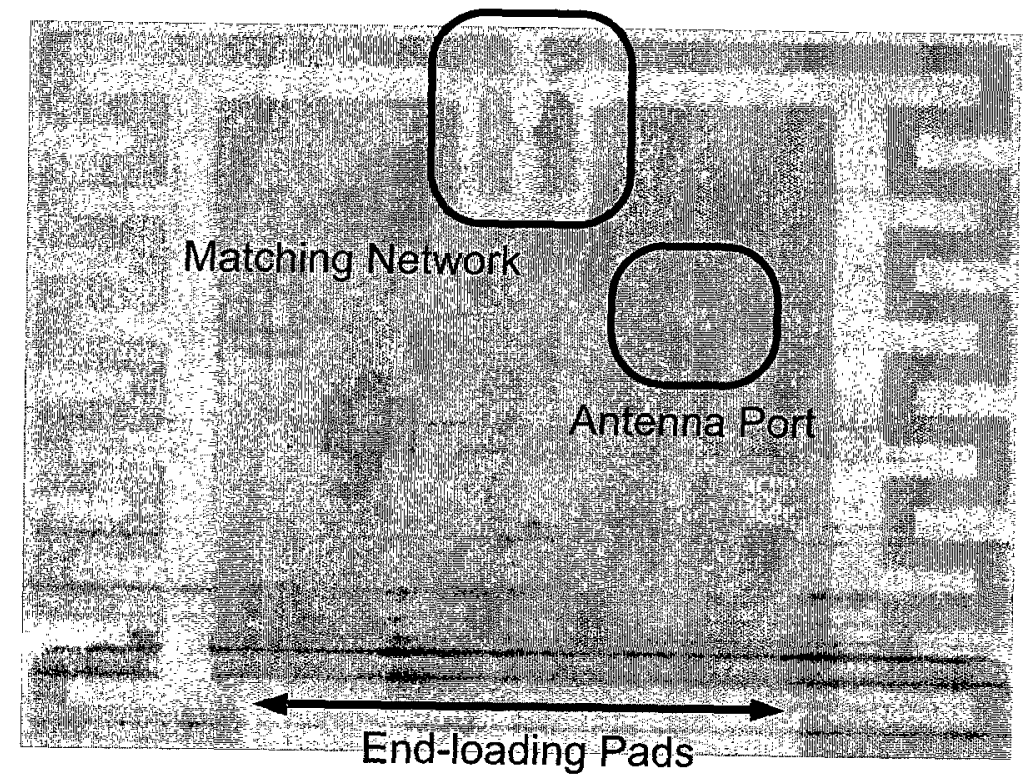

(b)

Figure 7.12: Micrograph of fabricated SoP antenna: (a) top; and (b) bottom view. 


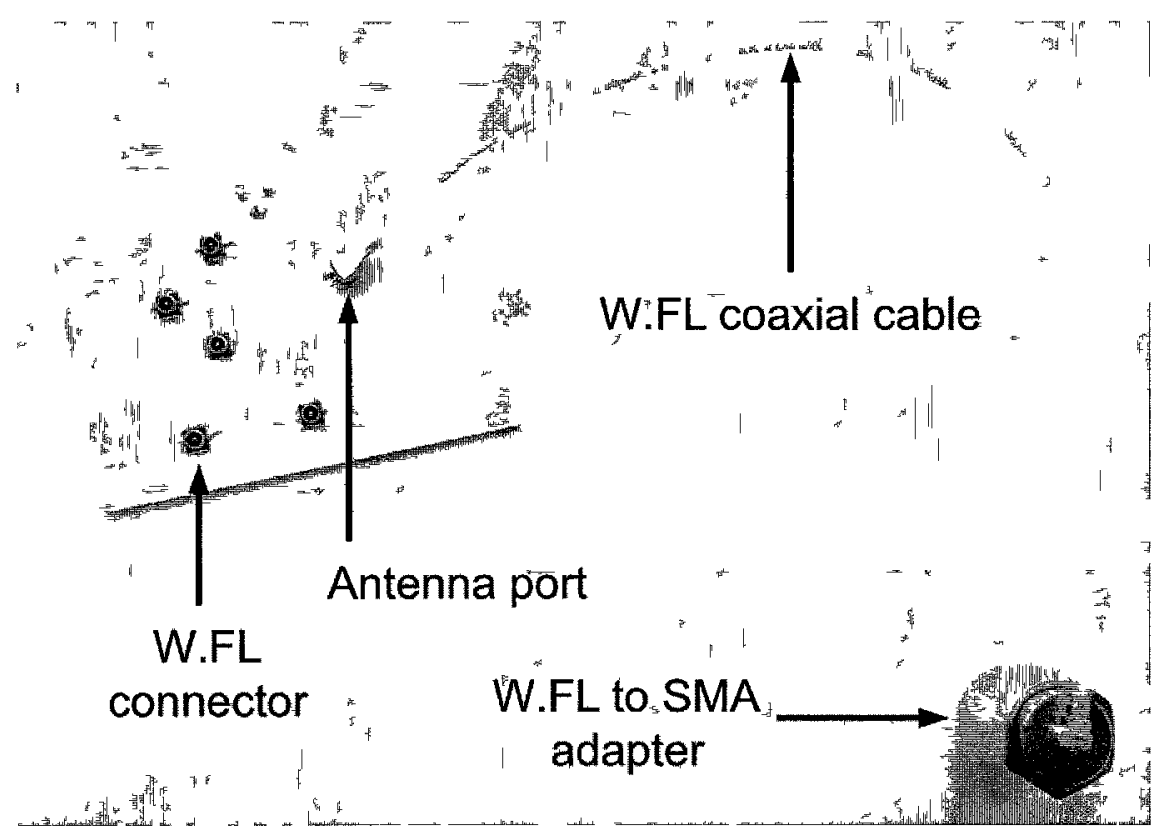

Figure 713 GPS module configured for dipole return loss measurement

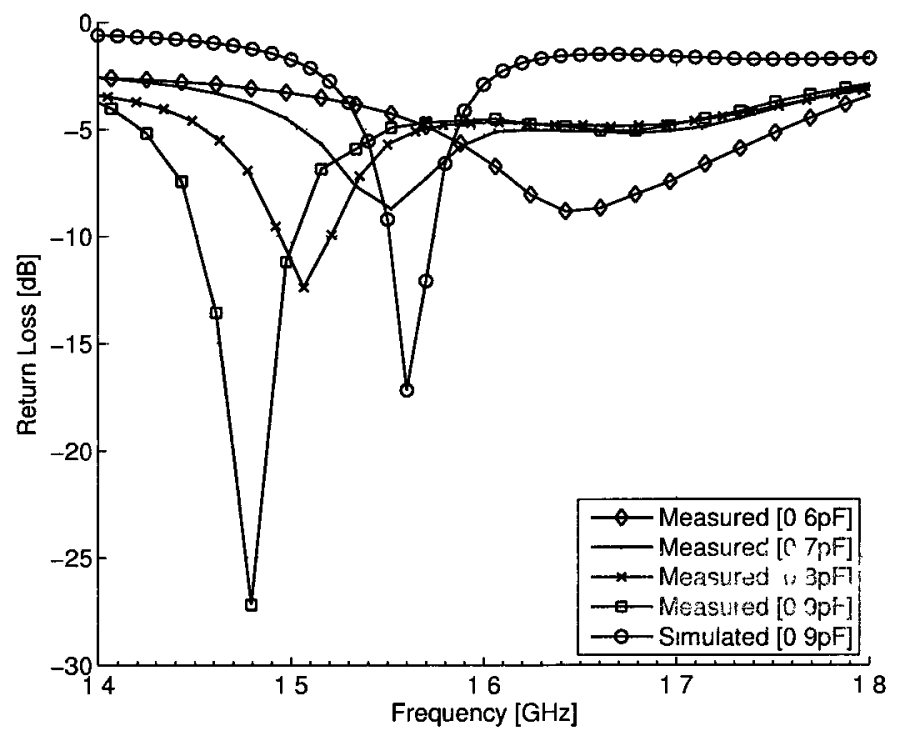

Figure 714 Measured antenna return loss for varıous values of matching capacitors. 
module were not simulated. Therefore, coupling to the numerous vias and ground planes was not accounted for. Of the remaining cases, a capacitance of $0.8 \mathrm{pF}$ provides the best in-band match and a minimum $\mathrm{S}_{11}$ of $-12 \mathrm{~dB}$ at $1.507 \mathrm{GHz}$. This configuration was used to obtain radiation patterns in the two principle planes. End-loading of the antenna arms would not be helpful since its effect would be to lower the resonant frequency instead of raising it. The anechoic chamber where the measurements were conducted is shown in Fig. 7.15. It's located within the David Florida Laboratory, which is part of the Canadian Space Agency facilities at CRC. A WR560 open-ended waveguide was used as the transmit antenna and NSI2000 V4.6.3 software controlled the calibration and data collection. A near-to-far-field transform was automatically performed in software.

The Antenna-Under-Test (AUT) is held securely by a foam base, which in turn is attached to a wooden "cartwheel". The AUT and cartwheel are then mounted on a pedestal that places the AUT at the center of the rotational axis of the positioner. It can be seen that the positioner holding the AUT obstructs the backwards view. Therefore, data in this range is not accurate and will be greyed out in the following graphs. Pattern measurements were obtained at $1.507 \mathrm{GHz}, 1.525 \mathrm{GHz}, 1.555 \mathrm{GHz}$ and $1.575 \mathrm{GHz}$.

In general, the radiation patterns in both planes are satisfactory. In the azimuth plane, the required low directivity is observed with cross-pol levels that are about $10 \mathrm{~dB}$ down. In the elevation plane, the pattern is more directive with nulls near the expected locations of 0 and 180 degrees. No rippling is observed, which would indicate that there is minimum spurious radiation of the coaxial feeding cables. The highest gain $(-0.8 \mathrm{dBi})$ and radiation efficiency $(26 \%)$ are observed at $1.55 \mathrm{GHz}$ and $1.525 \mathrm{GHz}$, respectively. 


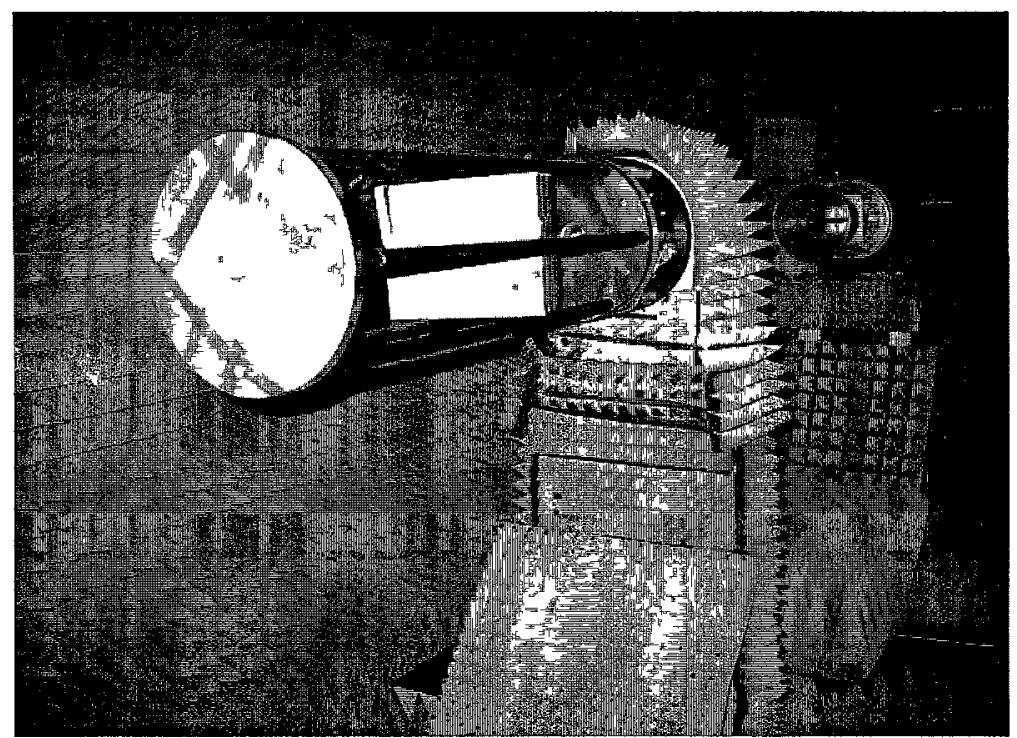

(a)

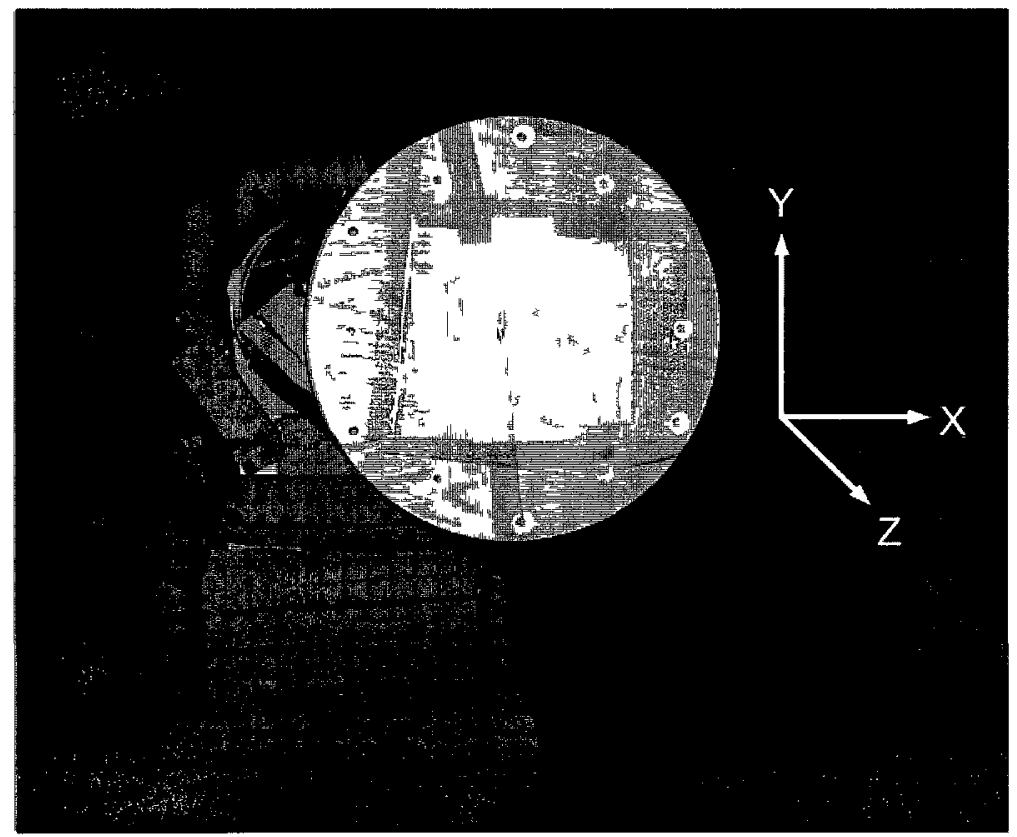

(b)

Figure 7 15: Photographs of anechoic chamber used to obtain radiation patterns. Image showing (a) both antennas; and (b) close-up of antenna under test 


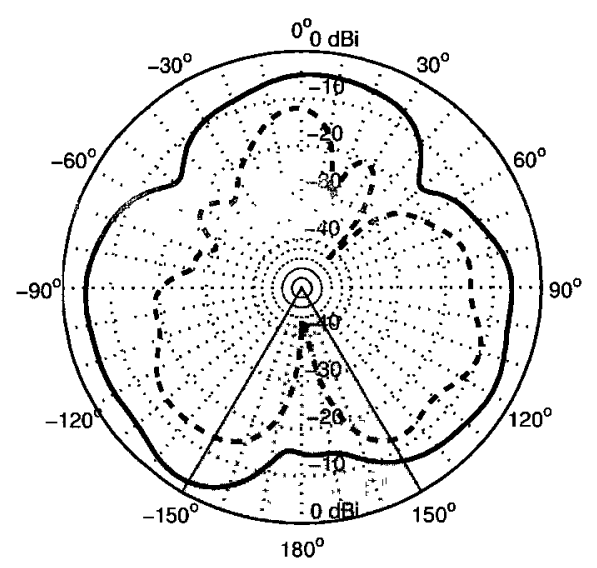

(a) $1.507 \mathrm{GHz}$

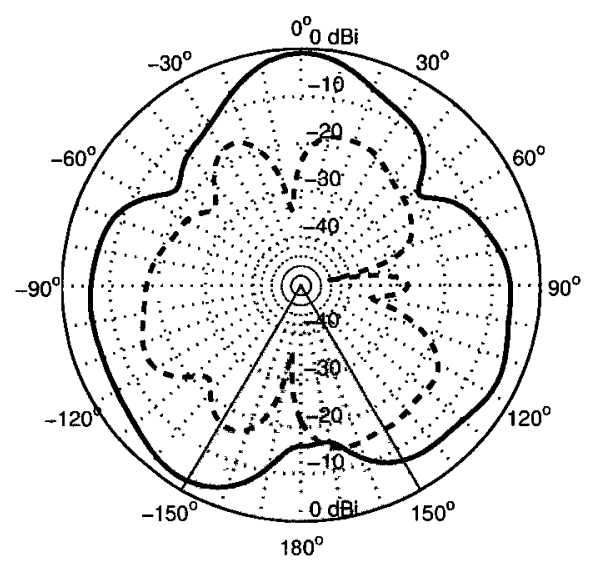

(c) $1.55 \mathrm{GHz}$

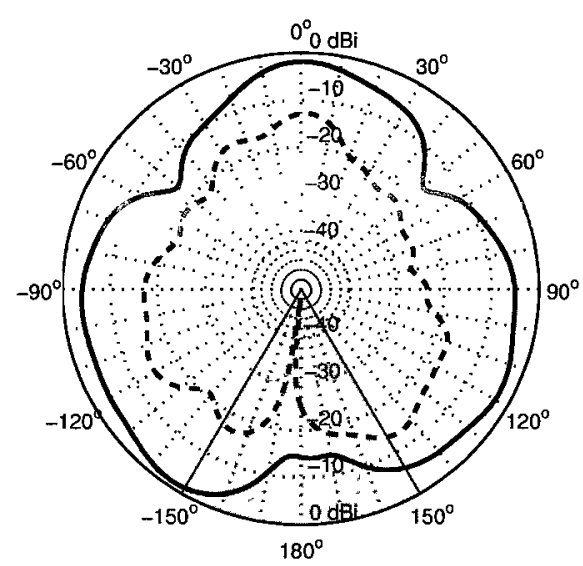

(b) $1.525 \mathrm{GHz}$

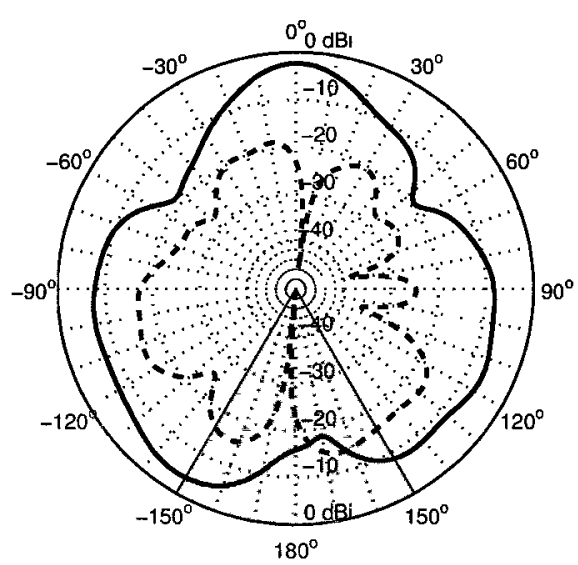

(d) $1.575 \mathrm{GHz}$

Figure 7.16: Experimental azimuth (Z-Y plane) radiation patterns. Co-pol (solid line) and cross-pol (dashed line) results. 


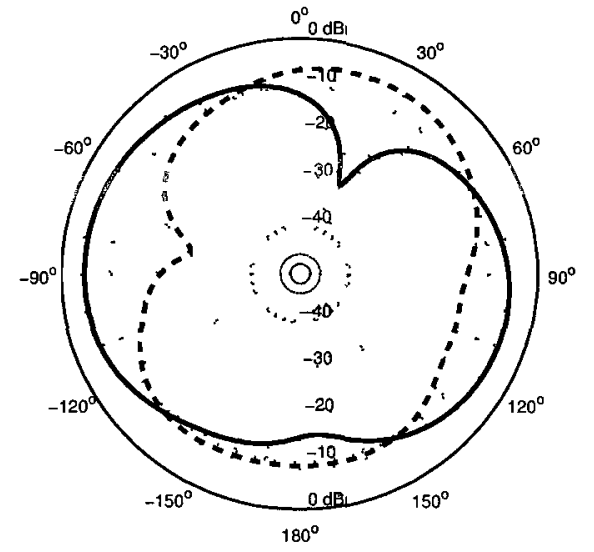

(a) $1.507 \mathrm{GHz}$

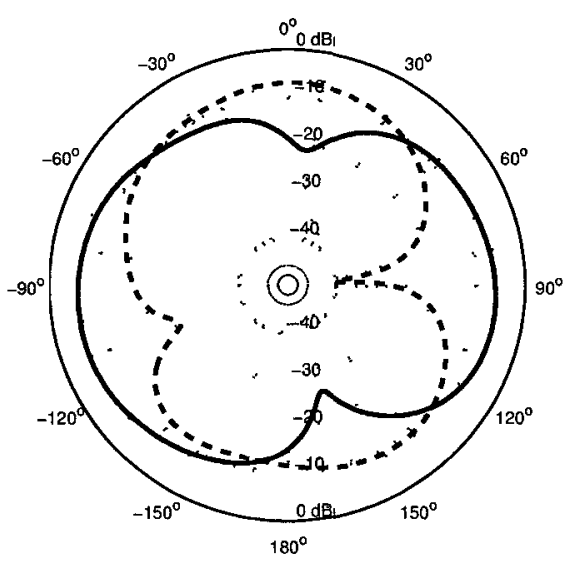

(c) $1.55 \mathrm{GHz}$

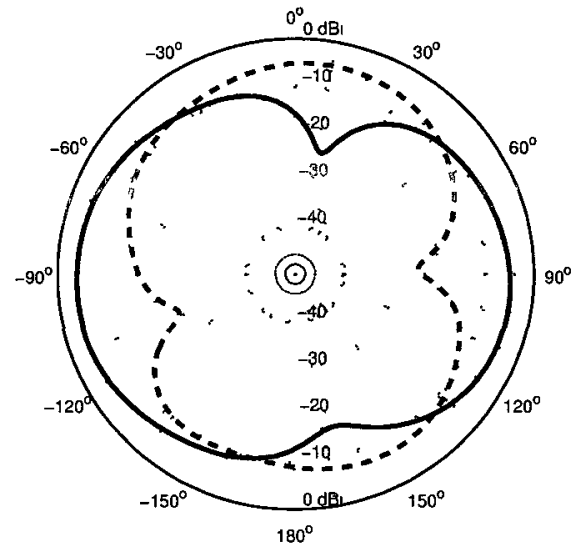

(b) $1.525 \mathrm{GHz}$

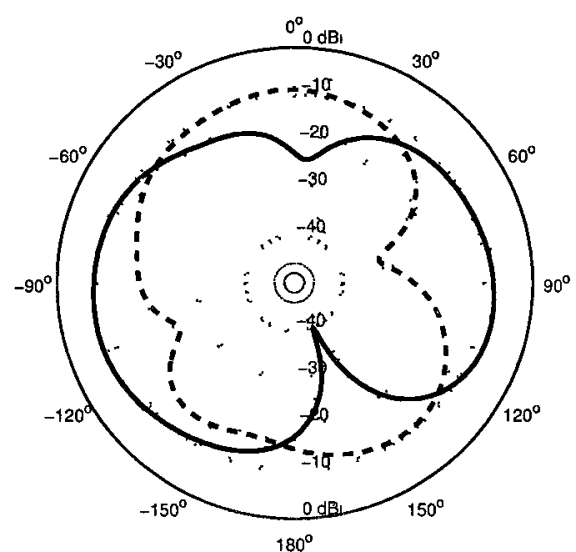

(d) $1575 \mathrm{GHz}$

Figure 7.17: Experimental elevation (Y-X plane) radiation patterns. Co-pol (solid line) and cross-pol (dashed line) results. 
To create a more meaningful comparison of gains and radiation efficiencies, passives of finite $\mathrm{Q}$ must be modeled. As mentioned earlier, $\mathrm{Q}$ of passive matching elements has an important effect on overall performance of the dipole (in this discussion, Q refers to unloaded Q). Since Q values can't be used directly by HFSS, they must first be converted to equivalent parallel resistances with the use of Equation 3.4. Where the Q's are typical values obtained from manufacturers datasheets. The model of Fig. 7.9(a) was augmented with these resistances of $32 \mathrm{k} \Omega$ and $2 \mathrm{k} \Omega$ that correspond to Q's of 300 (for each capacitor) and 70 (for the inductor), respectively. This model also shows that only the top layer metal is incorporated in the simulation since including all layers would be too computationally demanding.

The radiation efficiency is defined as the ratio of the radiated power to the accepted power given by Equation 7.1 [58].

$$
e=\frac{P_{r a d}}{P_{a c c}}
$$

where, $P_{\text {rad }}$ is the radiated power in watts and $P_{a c c}$ is the accepted power in watts. Therefore, all losses are accounted for including miss-match loss, conductor loss, dielectric loss and matching component loss. A comparison of the simulated and measured peak gains and efficiencies is provided in Table 7.3.

The tabulated figures show reasonable agreement between the simulated lossy and experimental results. In terms of radiation efficiency, the agreement is highest at lower frequencies because that is where experiments indicate the antenna to be resonant. At all frequencies, blockage caused by the positioner reduces the amount of radiated power received in that direction and thus efficiency suffers. Of note, at $1.55 \mathrm{GHz}$ the simulated efficiency is $38 \%$, compared to $65 \%$ when losses of the 
Table 7.3: A comparison of radiation efficiencies and peak gains

\begin{tabular}{|l|c|c|c|c|}
\hline \multicolumn{1}{|c|}{ Frequency [GHz] } & 1.507 & 1.525 & 1.55 & 1.575 \\
\hline Parameter & & & & -1.7 \\
\hline Peak Gain [dBi] [Simulated Lossless] & -5.2 & -4.4 & -3.0 & -2.7 \\
\hline Peak Gain [dBi] [Simulated Lossy] & -5.6 & -4.7 & -3.38 & -2.1 \\
\hline Peak Gain [dBi] [Measured] & -5 & -1.8 & -0.8 & -2.3 \\
\hline Efficiency [\%] [Simulated Lossless] & 25 & 31 & 42 & 55 \\
\hline Efficiency [\%] [Simulated Lossy] & 23 & 28 & 38 & 50 \\
\hline Efficiency [\%] [Measured] & 21 & 26 & 19 & 12.5 \\
\hline
\end{tabular}

matching components are not included. In all cases the miniature W.FL connectors, cables, and SMA adapter contribute to the losses. The positioner can also be acting as a deflector that increases the directivity of the antenna, which may explain why the measured peak gain values are about $3 \mathrm{~dB}$ higher than expected at $1.525 \mathrm{GHz}$ and $1.55 \mathrm{GHz}$, and why the measured efficiency values are lower than expected at $1.55 \mathrm{GHz}$ and $1.575 \mathrm{GHz}$. Other possible sources of error that contribute to the disagreement include $\mathrm{Q}$ tolerances of the matching components (that can vary by several percent) and losses of the conductive epoxy. In addition, the insertion losses, however small, of the balun also reduce the gain and efficiency of the dipole.

As a final comparison, Fig. 7.18 presents the simulated lossy and measured co-pol radiation patterns at $1.575 \mathrm{GHz}$.

\subsection{Summary and Contribution}

This chapter has presented a novel dipole suitable for integration in the GPS frontend module of this work. Two series capacitors and one parallel inductor are used 


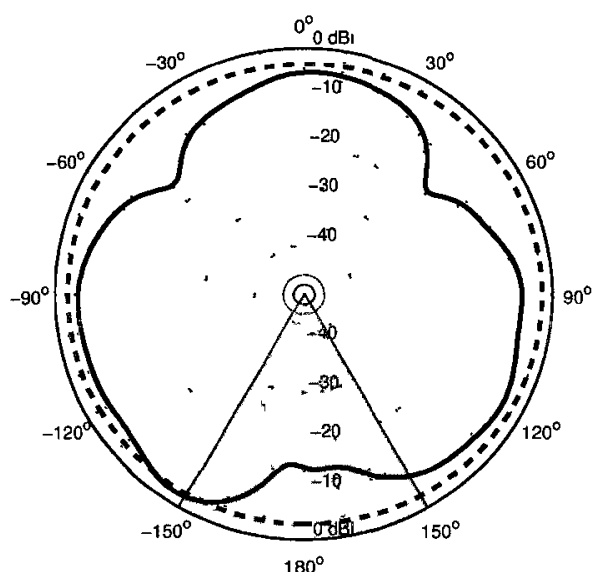

(a) Azımuth (Z-Y plane)

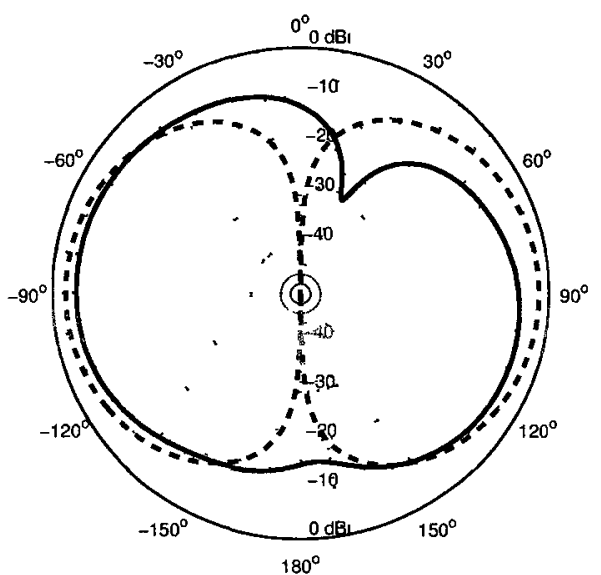

(b) Elevation (Y-X plane)

Figure 7.18: Comparison of principal plane radiation patterns at $1.507 \mathrm{GHz}$. Measured (solid line) and simulated (dashed line) results: (a) azimuth; and (b). Elevation

for the matching network to create a good impedance match and radiation efficiency at $1.55 \mathrm{GHz}$. A lumped element balun is used to convert the dipole's output from differential to single ended. The dipole-balun combination only occupies the module perimeter, leaving $40 \%$ of the total area available for use by remaining receiver components. The measured results are in good agreement with the simulated data and possible sources of error have been discussed. The specific publication arising from the work contained in this chapter includes one conference paper [9]. To prove its high performance, a comparison with commercial antennas from leading corporations is used in Table 7.4.

Based on performance only, the antenna presented here is competitive with similar commercial products. Its competitiveness increases when the overall size of the antennas is considered since the commercial examples compared here all require a large 
Table 7.4: Comparison with Commercial GPS Antennas

\begin{tabular}{|l|c|c|c|c|}
\hline Item & This Work & Johnson & Antenova & Taoglas \\
\hline Model No. & - & 1575AT43A40 & A10137-D & GLA.02 \\
\hline $\begin{array}{l}\text { Center Freq. } \\
{[\mathrm{MHz}]}\end{array}$ & 1550 & 1575 & 1575 & 1575 \\
\hline $\begin{array}{l}\text { Bandwidth } \\
{[\mathrm{MHz}]}\end{array}$ & 20 & 40 & 30 & 20 \\
\hline $\begin{array}{l}\text { Peak Gain } \\
\text { [dBi] (max) }\end{array}$ & -0.8 & -1.5 & -1.5 & 1.34 \\
\hline $\begin{array}{l}\text { Average Effi- } \\
\text { ciency [\%] }\end{array}$ & 19 & - & 70 & 65 \\
\hline Polarization & linear & linear & linear & linear \\
\hline $\begin{array}{l}\text { Size (Includ- } \\
\text { ing GND) } \\
\text { [mm x mm] }\end{array}$ & $24 \times 32$ & $20 \times 49.5$ & $20 \times 60$ & $40 \times 80$ \\
\hline $\begin{array}{l}\text { Relative Area } \\
\text { [\%] }\end{array}$ & 100 & 129 & 156 & 416 \\
\hline
\end{tabular}


external ground plane to function properly. The smallest example is still $29 \%$ larger than this work, while the largest is over four times larger. To the author's knowledge, no other antenna demonstrates such good performance for GPS applications in such a small package as the design presented here. The results of this chapter constitute the partial fulfillment of the fourth thesis contribution, namely the co-design and aggressive miniaturization of a SoP receiver front-end with antenna. 


\section{Chapter 8}

\section{SoP Design of an L-Band Miniature Receiver Module for GPS}

The work contained in this chapter represents the completion of the fourth and final thesis objective. Namely, the SoP integration of a GPS receiver front-end with antenna. Fig. 8.1, presents these devices and their locations in the receiver chain. All the necessary active components of the receiver will be described but first the

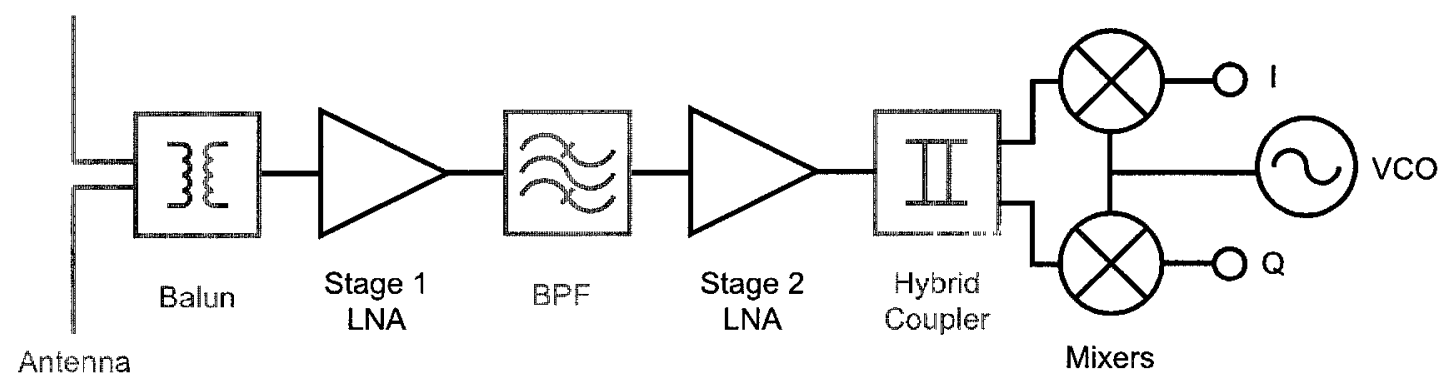

Figure 8.1: Active components of the miniature receiver module (MRM).

specifications and requirements will be discussed. 


\subsection{GPS Specifications}

The GPS signal from the satellite primarily uses the $1575.42 \mathrm{MHz}$ RF carrier. DirectSequence Spread Spectrum (DSSS) modulation is used with binary phase-shift keying (BPSK) encoding. Typical signal power at the receiver is extremely low, about $-130 \mathrm{dBm}$ which is actually below the thermal noise floor as calculated from Equation 8.1 where a channel bandwidth of $2.046 \mathrm{MHz}$ is used.

$$
\text { Noise Floor }=-174 \frac{d B m}{H z}+10 \log _{10}\left(2.046 \times 10^{6}\right)=-110.9 \mathrm{dBm}
$$

If the receiver and transmitter are synchronized then the signal can be recovered by de-spreading and integrating over a long period of time that results in a processing gain of several decibels [119]. In this way the required Signal-to-Noise Ratio (SNR) can be achieved. The remaining specifications for a GPS signal are summarized in Table 8.1.

Table 8.1: GPS Specifications

\begin{tabular}{|c|c|}
\hline Specification & Value \\
\hline Carrier Frequency & $1575.42 \mathrm{MHz}$ \\
\hline Power & $-130 \mathrm{dBm}$ \\
\hline Data rate $(\mathrm{Rb})$ & $50 \mathrm{bps}$ \\
\hline Channel Bandwidth & $2.046 \mathrm{MHz}$ \\
\hline Modulation & DSSS+BPSK \\
\hline
\end{tabular}




\subsection{Receiver Requirements}

A detailed analysis of the requirements of a complete GPS receiver are presented in [119]. Based on this study, only the requirements relevant to a receiver front-end are discussed here and include gain, noise, and image rejection performance.

\subsubsection{Gain}

Due to a GPS signal's low power, a total system gain of approximately $100 \mathrm{~dB}$ is required. In modern receiver designs, only $10 \%$ to $40 \%$ of that gain is generated by the front-end with the remainder provided by the digital baseband circuitry [120]. Therefore, a front-end with between $20 \mathrm{~dB}$ and $40 \mathrm{~dB}$ gain would be sufficient for this work. Cascaded amplifiers with moderate gain can be used to maintain good linearity and give the option of realizing variable gain.

\subsubsection{Noise Figure}

Good Noise Figure (NF) performance of a GPS receiver is important in ensuring that the minimum sensitivity is low enough to reduce the likelihood of tracking errors. The receiver sensitivity can be calculated from Equation 8.2 [119].

$$
\text { Sensitivity }[d B m]=\left(\frac{C}{N_{0}}\right)_{\min }\left[\frac{d B m}{H z}\right]+N_{0}\left[\frac{d B m}{H z}\right]+N F[d B]
$$

where, $\mathrm{N}_{0}$ is the thermal noise floor $(-174 \mathrm{dBm} / \mathrm{Hz}), N F$ is the noise figure of the receiver, and $\frac{C}{N_{0}}$ is the carrier-to-noise ratio which is the SNR normalized to a $1 \mathrm{~Hz}$ bandwidth. Here, the target $\frac{C}{N_{0}}$ and $\mathrm{NF}$ are $37 \mathrm{dBm} / \mathrm{Hz}$ and $3 \mathrm{~dB}$, respectively. With these specifications, the receiver's sensitivity can extend down to $-134 \mathrm{dBm} / \mathrm{Hz}$. As is 
well know, the NF of the receiver is largely determined by the noise performance of the first element in a cascaded system. Here, the antenna-balun combination along with the first stage LNA can be grouped together to form an active antenna. This block constitutes the first element in the receiver presented here and is shown in Fig. 8.2 .

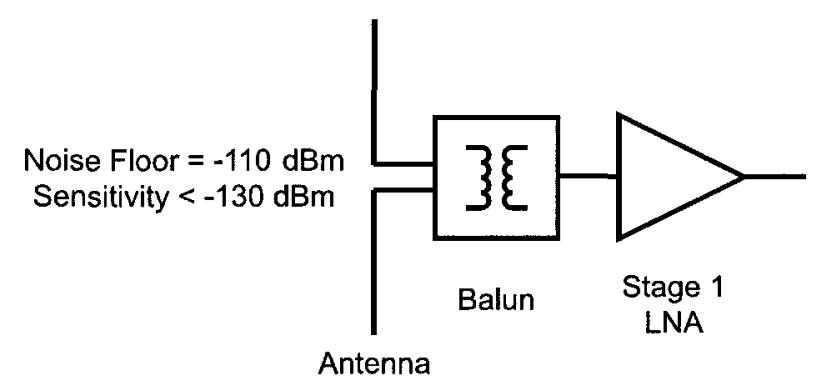

Figure 8.2: Block diagram of the active antenna which constitutes the first element in the receiver.

An estimate of the active antenna's NF can be obtained by recalling the balun's insertion losses from Chapter 6 and applying Friis' formula for noise. This data along with the NF and gain of a typical LNA is listed in Table 8.2. Being a passive device,

Table 8.2: Active Antenna Specifications

\begin{tabular}{|c|c|c|}
\hline Component & Gain [dB & NF [dB] \\
\hline Balun & -1.17 & 1.17 \\
\hline LNA & 15 & 2.2 \\
\hline
\end{tabular}

the balun's NF is equal to the sum of its insertion losses at the output ports. The balun and LNA form a cascaded system that has a total NF of $2.1 \mathrm{~dB}$ when Friis' formula for noise is applied. This result gives a margin of $0.9 \mathrm{~dB}$ to the maximum desired $\mathrm{NF}$ of $3 \mathrm{~dB}$. 


\subsubsection{Image Rejection Ratio}

Quadrature downconversion is required for GPS communication since the two sides of the BPSK spectra carry different information that must be separated before recombining at IF. Several methods exist to reduce the amount of noise contribution from the image band. The chosen solution will be discussed later but for now it is important to note that the level of rejection is dependant on the amplitude and phase balance of the $\mathrm{I} / \mathrm{Q}$ branches. This relationship is called the Image Rejection Ratio (IRR) and is defined by Equation. 8.3 [67].

$$
I R R=\frac{1+2 A \cos (\theta)+A^{2}}{1-2 A \cos (\theta)+A^{2}}
$$

where, $\mathrm{A}$ is the amplitude mismatch and $\theta$ is the phase mismatch. Plotting this relationship for phase errors between 0.1 and 100 degrees and amplitude errors of 0 to $2 \mathrm{~dB}$ yields the curves in Fig. 8.3. Based on these curves, a realistically achiev-

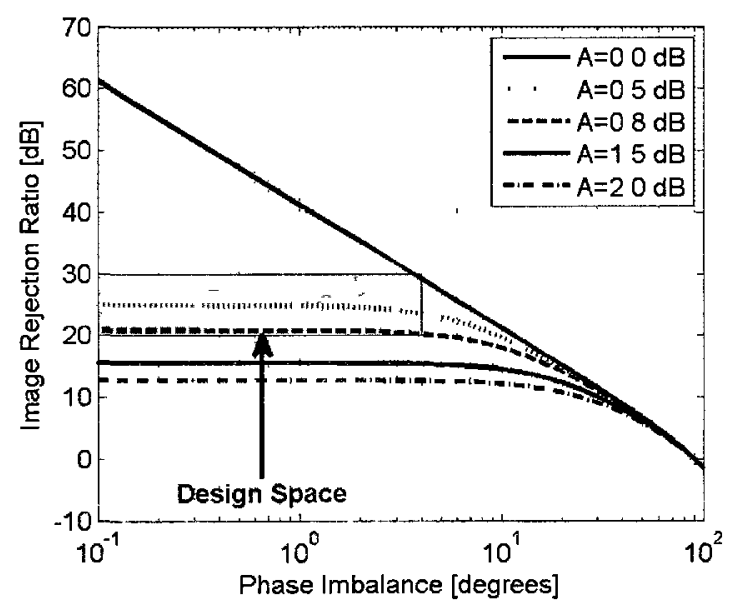

Figure 8.3: Image rejection ratio for different values of phase and amplitude imbalance from Equation 8.3. 
able IRR lies in the range of 20 to $30 \mathrm{~dB}$. The shaded region of Fig. 8.3 indicates that to achieve this specification, the amplitude imbalance must be less than $0.8 \mathrm{~dB}$ and simultaneously the phase imbalance must be below 3 degrees. A higher IRR would require a more complicated IRM with active amplitude balancing composed of components with extremely tight tolerances. A summary of the derived receiver requirements is provided in Table 8.3. Next, the design of the complete miniature

Table 8.3: Summary of Receiver Requirements

\begin{tabular}{|c|c|}
\hline Specification & Value \\
\hline System Gain & $>90 \mathrm{~dB}$ \\
\hline$\frac{C}{N_{0}}$ & $37 \mathrm{~dB}$ \\
\hline Noise Figure & $<3 \mathrm{~dB}$ \\
\hline Image Rejection Ratio & $>20 \mathrm{~dB}$ \\
\hline
\end{tabular}

receiver module (MRM) is discussed.

\subsection{MRM System Architecture}

The MRM uses a superheterodyne (or heterodyne) system architecture that is extensively employed in commercial products because it reduces the $Q$ requirement of the channel select filter by translating the channel bandwidth to a lower intermediate frequency (IF) [67]. The translation or downconversion is carried out by way of a pair of mixers that multiply the incoming RF signal with a local oscillator (LO) signal to produce I and Q IF outputs. In this implementation, the mixers are located on the MRM but the LO is fed from an external source. 


\subsubsection{Basic Functionality}

Before a detailed schematic of the MRM is presented, its basic functionality is discussed in reference to Fig. 8.1 that shows a simplified representation of the MRM. The antenna presented in chapter 7 is the first component in the chain that captures the RF signal and converts it to a voltage difference across its terminals. The balun from chapter 6 then translates this differential voltage to a single-ended signal referenced to a $50 \Omega$ impedance that is passed to the first stage LNA. The LNA is placed right after the antenna-balun combination because it is well known that the first stage (in a cascaded system) has a dominating influence on the noise performance of the system [23]. That is, the high gain and low noise figure of the LNA will work to diminish the noise added by later stages like the BPF. The filter is the next stage in the receiver. In fact, the schematic and layout of the MRM will show that both filters presented in chapter 4 have been included with an ability to select one or the other. The next component in the receiver is the second stage LNA that is by default enabled but the MRM has the provision to bypass this gain stage if it is not required.

After the second stage LNA, the signal reaches the downconversion stage discussed earlier that also implements part of the IRM. The necessary quadrature signals can either be generated in the LO or RF path. Here, the phase shift is applied in the RF path with the use of the 90-degree hybrid presented in Chapter 5. This method avoids the need for a polyphase filter that is typically used to create the phase shift but also brings with it many tradeoffs [67]. To create an IRM, one of two popular circuits can be employed. The Weaver and Hartley architectures differ in the way the image is canceled. Whereas the Hartley circuit uses a second 90-degree phase shift block to create an image signal of opposite polarity, the Weaver circuit uses another complete quadrature mixing stage to achieve the same result. Therefore the 
Hartley circuit is much simpler but compared to the Weaver it is more sensitive to amplitude and phase imbalances. Given the fact that a 90-degree hybrid with good phase and amplitude balance has already been demonstrated, these drawbacks can be minimized. The final IRM configuration is shown in Fig. 8.4.

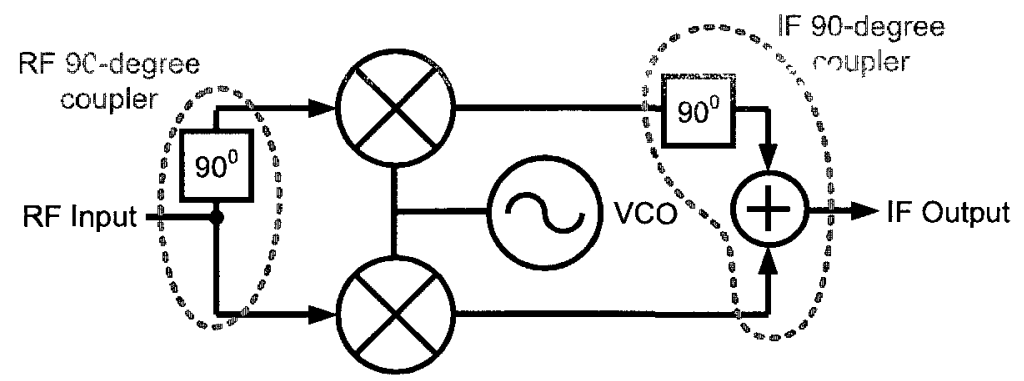

Figure 8.4: A quadrature downconverter with a Hartley image reject mixer and RF path quadrature generation.

Of course, as part of the quadrature generation process, the coupler also divides the RF signal and removes the need for an additional component for this purpose. The quadrature phases are then mixed down with the LO signal to create I and Q intermediate frequency (IF) signals that are routed from the MRM and finally combined by another 90-degree hybrid coupler. The latter coupler is an external component because at IF frequencies its size is too large to incorporate on the MRM. With the basic functionality of the MRM described, a detailed schematic representation is contemplated next.

\subsubsection{Detailed Circuit Schematic}

A detailed schematic of the full receiver is shown in Fig. 8.5 with all components labeled. The input to the receiver chain can either be provided from the antennabalun combination (labeled as "BALUN_OUT") or from an external port (labeled as 


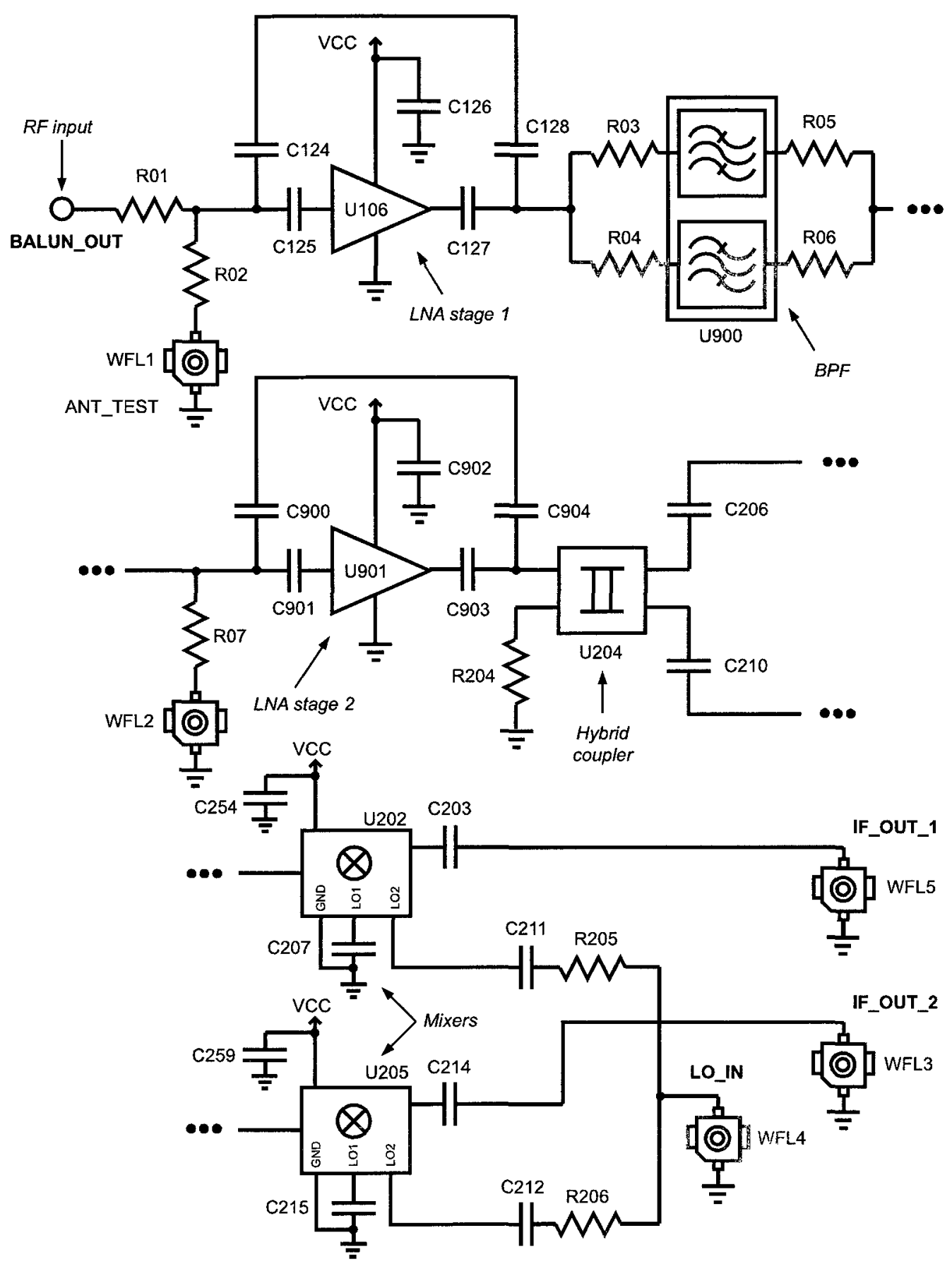

Figure 8.5: Detailed schematic of MRM without antenna and balun stages. 
"ANT_TEST"). All external ports are implemented with W.FL connectors, which were first presented in chapter 7. The first stage LNA, U106, is surrounded by decoupling capacitors and a bypass path can be used for debugging purposes. The output of this LNA is sent to a filter bank, U900, that contains single copies of the second-order and fourth-order filters of chapter 4. Either filter can be selected by appropriately choosing resistors R03 to R06 to be either open or $0 \Omega$.

The output of the filter can either be directed to the second stage LNA or to an output port (WFL2) for debugging purposes. The second stage LNA is identical to the first and is enabled by default but again can be bypassed if desired. The next component in the chain is the 90-degree hybrid coupler (U204) that has as its outputs quadrature signals for the mixers (U202 and U205). At this point it is crucial to keep both signal paths equal in length to maintain their relative phase shift. Similarly, the LO feed paths also must have the same length. The outputs of the mixers are sent to a pair of W.FL connectors (WFL3 and WFL5) so that they can be transferred to another module for further processing.

Power to the MRM module is supplied from an external source through one of the W.FL connectors (WFL6). A low-dropout power regulator (U305) is located on the MRM to ensure that the voltage and current remain stable during all circumstances. This circuit is shown in Fig. 8.6. In addition, three large valued capacitors (C302 to C304) are included at the output of the regulator to filter-out any low-frequency harmonics or other noise that may be present on the supply rail. Finally, a ferrite bead (B101) is placed in series with the power supply to block any RF leakage from the MRM. 


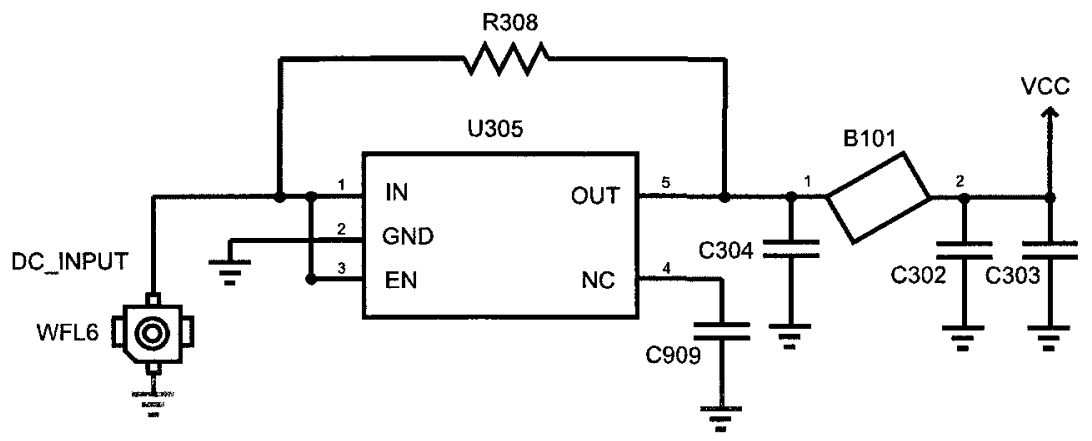

Figure 8.6: Low drop-out power regulator for MRM.

\subsection{MRM Layout Design}

The total area for the MRM is $32 \mathrm{~mm} \times 24 \mathrm{~mm}$ but some of this area has already been allocated to the antenna-balun combination presented in Chapter 7. In section 7.4.2 the layout of the antenna-balun was shown to occupy the perimeter of the MRM along with an interior rectangle (for the balun). Although the antenna only occupies one layer of the 8 layer substrate, all the layers overlapping it were designated as keep-out areas to prevent unwanted loading of the antenna. The unused area is dedicated to the remaining receiver components as shown in Fig. 8.7. The dedicated receiver area measures $20 \mathrm{~mm} \times 19 \mathrm{~mm}$ but, as already stated, one corner is reserved for the balun. The layout of the receiver proceeds in a counter clockwise direction beginning in the top left region near the balun and ending in the top right corner of the MRM. The placement of components follows the same order as the detailed schematic to minimize transmission line lengths. All the previously described LTCC components, including the BPFs, coupler, balun, and antenna are placed within the MRM unchanged. That is, no additional optimization of their dimensions is conducted to compensate for any de-tuning the MRM environment may induce. Instead, careful layout considerations are used to keep their performance almost identical to what was observed in their 
MRM

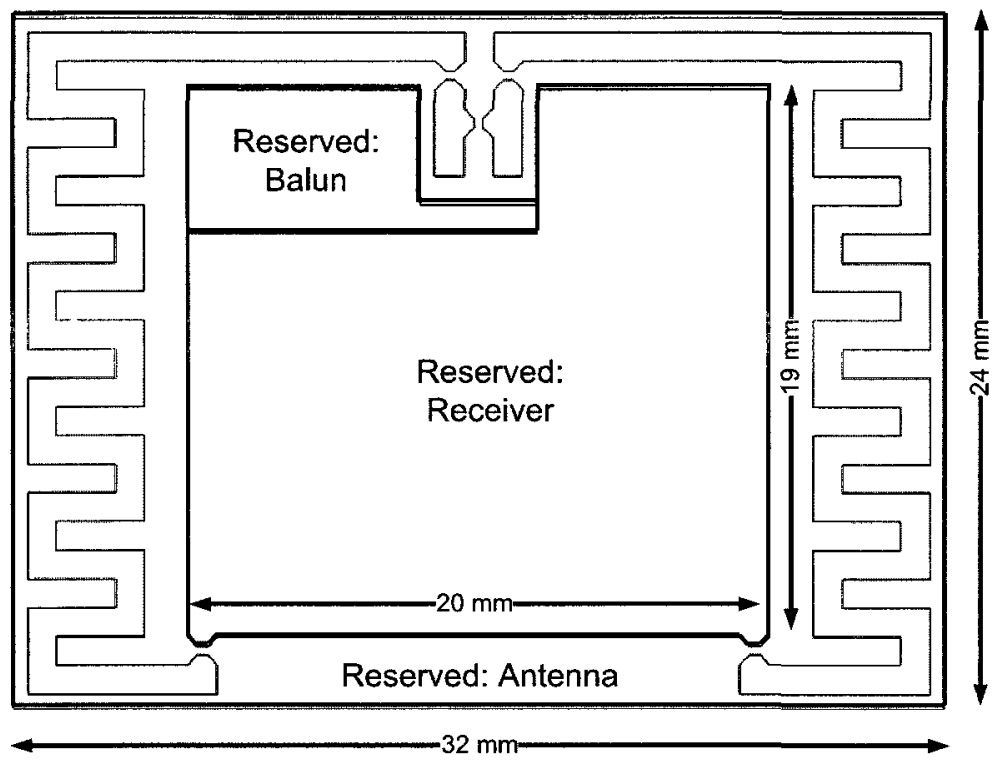

Figure 8.7: Area allocation for the various components of the MRM.

stand-alone implementations. The final locations of all the receiver components are displayed in Fig. 8.8 which shows a top view of the MRM. The antenna is only shown in Fig. 8.8 to provide perspective, it is not located on the same layer as the receiver electronics. Rather, the antenna is printed on the bottom layer of the MRM. This will be made more clear in section 8.6 where the fabricated MRM is presented. All the layers of the MRM are listed and described in Table 8.4. To enhance the electromagnetic shielding of the MRM, CPW transmission lines are used exclusively on L8. Internally, stripline interconnects are used on L5 with associated ground planes located on layers L7 and L3 creating a height of 4 layers. With this configuration layers L6 and L4 are left blank. The extra height increases the characteristic impedance of the structure and makes a $50 \Omega$ line width more reasonable. Layers L2 and L1 contain various ground planes while L1b, the exposed bottom layer, contains the antenna and 


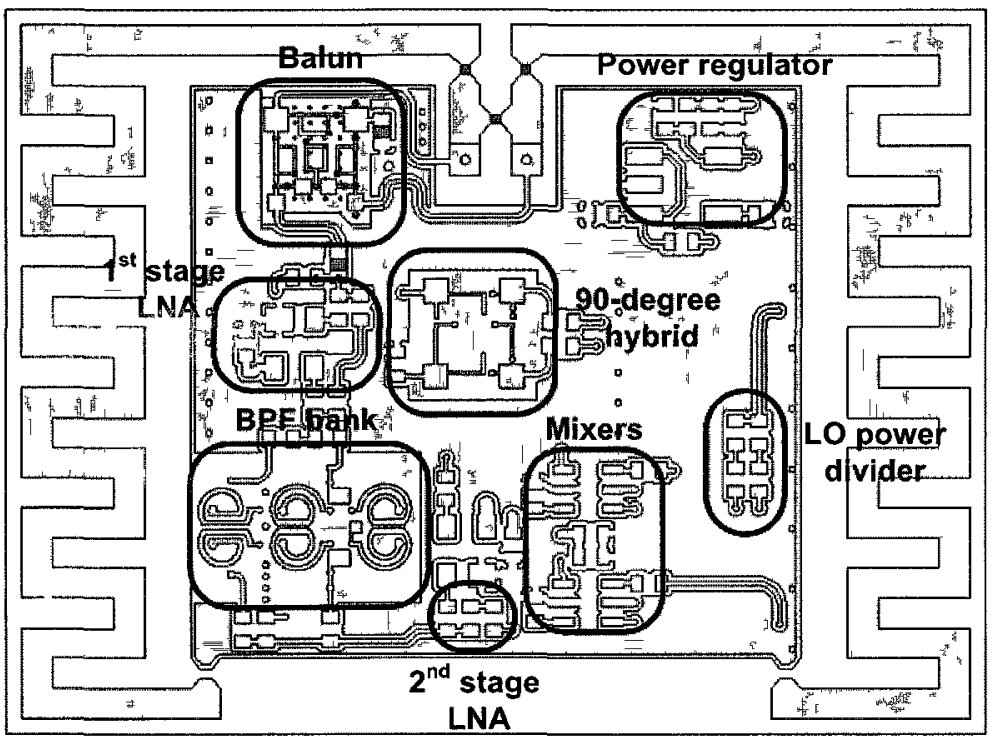

Figure 8.8: Locations of MRM components.

Table 8.4: MRM Layer Descriptions

\begin{tabular}{|c|c|}
\hline Layer & Description \\
\hline L8 & Top layer containing CPW lines and pads for all SMT components. \\
\hline L7 & Internal ground layer 1. \\
\hline L6 & Blank 1. \\
\hline L5 & Stripline layer. \\
\hline L4 & Blank 2. \\
\hline L3 & Internal ground layer 2 \\
\hline L2 & Internal ground layer 3. \\
\hline L1 & Partial ground layer for BPFs and balun. \\
\hline L1b & Bottom layer containing antenna and WFL connectors. \\
\hline
\end{tabular}


WFL connector pads. Selected metal layers are shown in Fig. 8.9.

The final metal layers shown in Fig. 8.9 also reveal a significant number of vias whose purpose it is to keep all ground planes at the same potential. Furthermore, some are placed around the embedded filters, coupler, and balun to increase their isolation. In the following section, active components are selected to meet the specifications outlined earlier. In summary, the MRM layout design resulted in the antenna and receiver front-end being tightly integrated without significant interference. The key enablers for achieving this were:

1. Strategic layout area allocation to ensure no overlap of receiver components and antenna (where high intensity electric fields exist).

2. Appropriate use of transmission line technologies (CPW, stripline) to enhance shielding.

3. Extensive use of vias and via fencing of sensitive embedded passives to reduce unwanted coupling and parasitic loading of resonators in particular.

\subsection{Active Component Selection}

The active components of the MRM are all implemented with commercial SMT devices. Alternatively, a custom RFIC could have been designed to combine all the discrete components on a single IC package to reduce their footprint and potentially power consumption as well. However, given a bulk CMOS process, such a design would likely not perform to the same level as a discrete implementation that takes advantage of specialized technologies. Furthermore, it would significantly increase the time required for design and measurement of the MRM. 

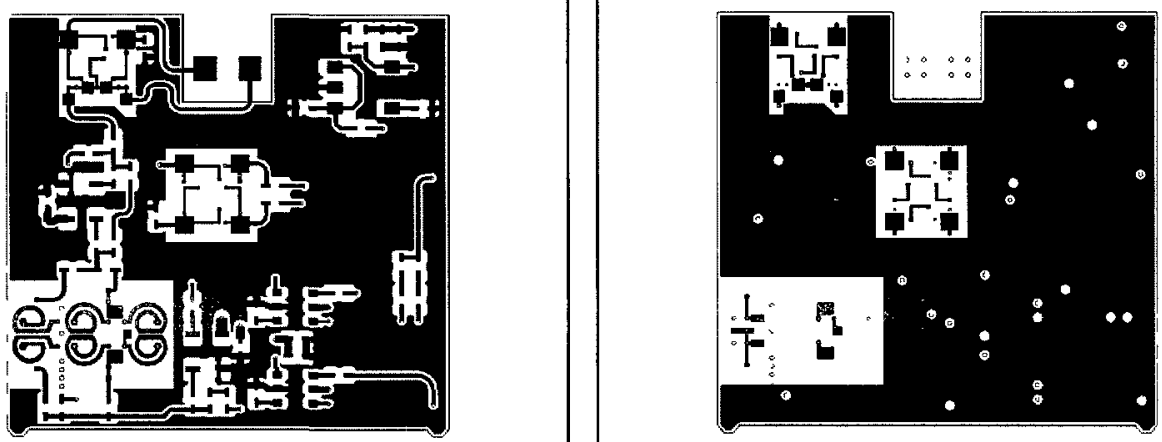

(a) L8

(b) L7

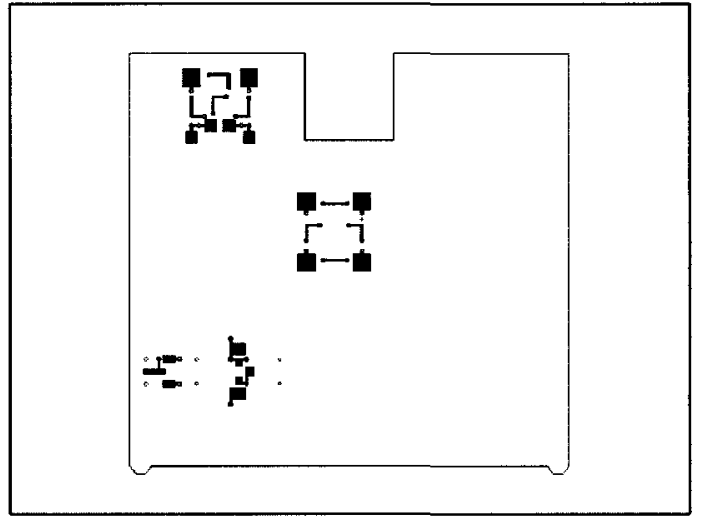

(c) L6

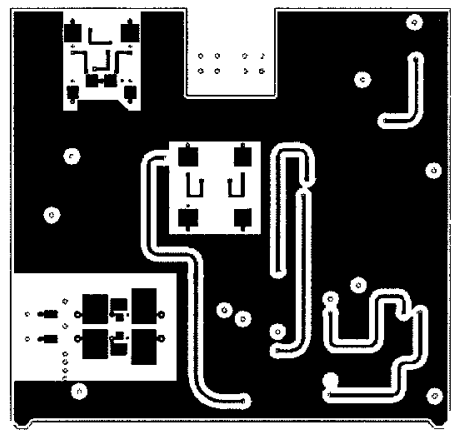

(d) L5

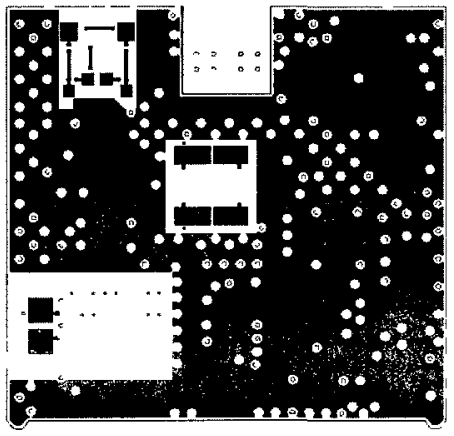

(e) L2

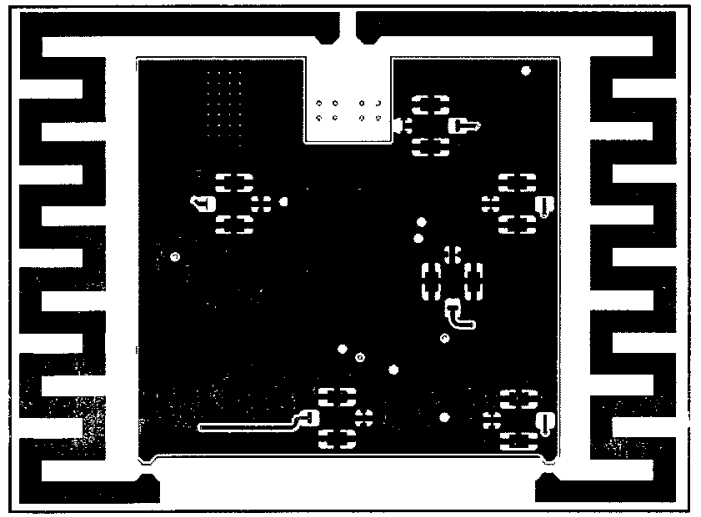

(f) L1b

Figure 8.9: Selected metal layers of the MRM. 
The SMT devices were selected to meet the stated receiver specifications and match as closely as possible those used by SkyWave Inc. in their commercial GPS receiver so that a fair performance comparison could be made. A total of five active components are required by the receiver with the most critical components being the LNAs and mixers. Silicon - Monolithic Microwave Integrated Circuit (Si-MMIC) amplifiers with $15 \mathrm{~dB}$ gain and $2.2 \mathrm{~dB}$ NF and Si-MMIC downconverters with $6 \mathrm{~dB}$ of conversion gain and a $10 \mathrm{~dB}$ NF were chosen. The gain of these devices totals about $36 \mathrm{~dB}$, which satisfies the gain requirement given earlier. The NF of the amplifiers is very low and should provide enough margin so that the overall NF is below $3 \mathrm{~dB}$. As described earlier, the IRR is limited by the amplitude and phase balances of the I and $\mathrm{Q}$ signal paths. In practice, the RF hybrid, mixers, and IF hybrid are the components that will determine the IRR. The RF hybrid is a known quantity but the mixers can't be perfectly matched due to fabrication tolerances. Similarly, the IF hybrid is a lumped element implementation where each of its inductors and capacitors have a 5\% tolerance. Finally, a low drop-out power regulator (U305) is also located on the module to provide a clean and stable supply voltage to the MRM. The full bill of materials is given in Table 8.5.

Table 8.5: MRM Bill of Materials

\begin{tabular}{|l|l|l|}
\hline Reference Designator & Description & Part No. \\
\hline \hline C206, C207, C210, C211, & CHIP CAP 0402 22 pF 5\% & GRM1555C1H220JZ01 \\
C212, C215, C254, C259, & 50 V COG & \\
C303, C900, C904, C908 & & \\
\hline
\end{tabular}


Table 8.5: MRM Bill of Materials

\begin{tabular}{|c|c|c|}
\hline Reference Designator & Description & Part No. \\
\hline C302, C304, C329 & $\begin{array}{l}\text { CHIP CAP } 04021 \mu \mathrm{F} \quad 10 \% \\
6.3 \mathrm{~V} \text { X5R }\end{array}$ & $\mathrm{N} / \mathrm{A}$ \\
\hline $\mathrm{C} 203, \mathrm{C} 214$ & $\begin{array}{l}\text { CHIP CAP } 0402 \quad 10 \mathrm{nF} 10 \% \\
10 \mathrm{~V} \text { X7R }\end{array}$ & N/A \\
\hline C909 & CHIP CAP $04020.01 \mu \mathrm{F}$ & $\mathrm{N} / \mathrm{A}$ \\
\hline $\begin{array}{l}\text { C124, C128, C125, C126, } \\
\text { C127, C901, C902, C903, } \\
\text { R308, R01, R02, R03, } \\
\text { R04, R05, R06, R07 }\end{array}$ & CHIP RES $04020 \Omega$ & $\mathrm{N} / \mathrm{A}$ \\
\hline $\mathrm{R} 204, \mathrm{R} 205, \mathrm{R} 206$ & $\begin{array}{l}\text { CHIP RES } 040249 \mathrm{R} 91 \% \\
1 / 16 \mathrm{~W}\end{array}$ & $\mathrm{~N} / \mathrm{A}$ \\
\hline B101 & $\begin{array}{l}\text { CHIP FERRITE BEAD } 0402 \\
1 \mathrm{~K} 25 \% 200 \mathrm{~mA} 0.1 \Omega\end{array}$ & BLM15HG102SN1 \\
\hline U106, U901 & $\begin{array}{l}\text { Si-MMIC-Amplifier in } \\
\text { SIEGET 25-Technology } \\
\text { SOT343-4 }\end{array}$ & BGA427 \\
\hline U202, U205 & $\begin{array}{l}\text { 3V Super Minimold } \\
\text { L-Band Si MMIC } \\
\text { Downconverter SOT363R }\end{array}$ & UPC2756TB-E3 \\
\hline
\end{tabular}


Table 8.5: MRM Bill of Materials

\begin{tabular}{|l|l|l|}
\hline Reference Designator & Description & Part No. \\
\hline WFL1, WFL2, WFL3, WFL4, & $\begin{array}{l}\text { RF, Coaxial connector, } \\
\text { SMT }\end{array}$ & W.FL-R-SMT-1 \\
\hline U305 & $\begin{array}{l}\text { Single Output LD0, } \\
100 \mathrm{~mA}, \text { Fixed(3.0 V), } \\
\text { Low Noise, High PSRR }\end{array}$ & TPS79230 \\
\hline U900 & $\begin{array}{l}\text { Custom, embedded LTCC } \\
\text { BPF (x2) }\end{array}$ & N/A \\
\hline U204 & $\begin{array}{l}\text { Custom, embedded } \\
\text { LTCC 90-degree hybrid } \\
\text { coupler }\end{array}$ & N/A \\
\hline
\end{tabular}

The discrete passives of the MRM are implemented with 0402 sized components. Although smaller 0201 versions are available they would make manually populating the MRM significantly more difficult. Another advantage of 0402 sized components is that they typically have lower tolerances than their 0201 counterparts. This is especially important for component pairs that need to be matched like resistors R205 and R206. For these in particular, ultra-low $1 \%$ tolerance components were selected.

The active components have small, 6-pin SOT packages that easily fit on the MRM. Although the filter bank and the 90-degree hybrid are embedded components, they have placeholders that appear on the bill of materials as U900 and U204 to make layout creation easier. 


\subsection{Fabricated MRM}

The MRM was fabricated using the same LTCC process as the previously presented filters, coupler, and balun. Images of the top and bottom of the MRM with labeled components are shown in Fig. 8.10. Here it is easy to see that the antenna metal

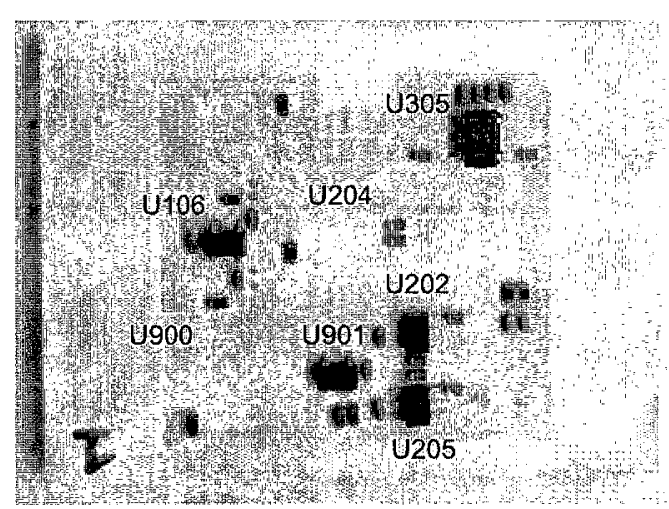

(a)

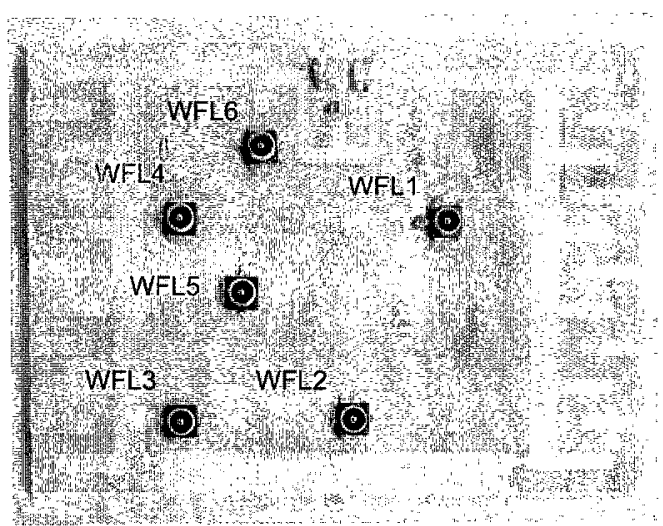

(b)

Figure 8.10: Micrograph of fabricated MRM with populated components: (a) top; and (b) bottom view.

occupies the bottom of the MRM along with all the WFL connectors. The SMT components and WFL connectors are placed on opposite sides of the module to declutter the measurement setup as much as possible.

\subsection{MRM Testing}

The test setup used to measure the MRM is shown in Fig. 8.11 where the larger circuit board is a SkyWave Inc. "Core Modem" (CM) transceiver. On the MRM, the second order BPF is enabled due to its lower insertion loss. The CM contains a receiver front-end similar to the MRM, however, it is significantly larger mainly due 


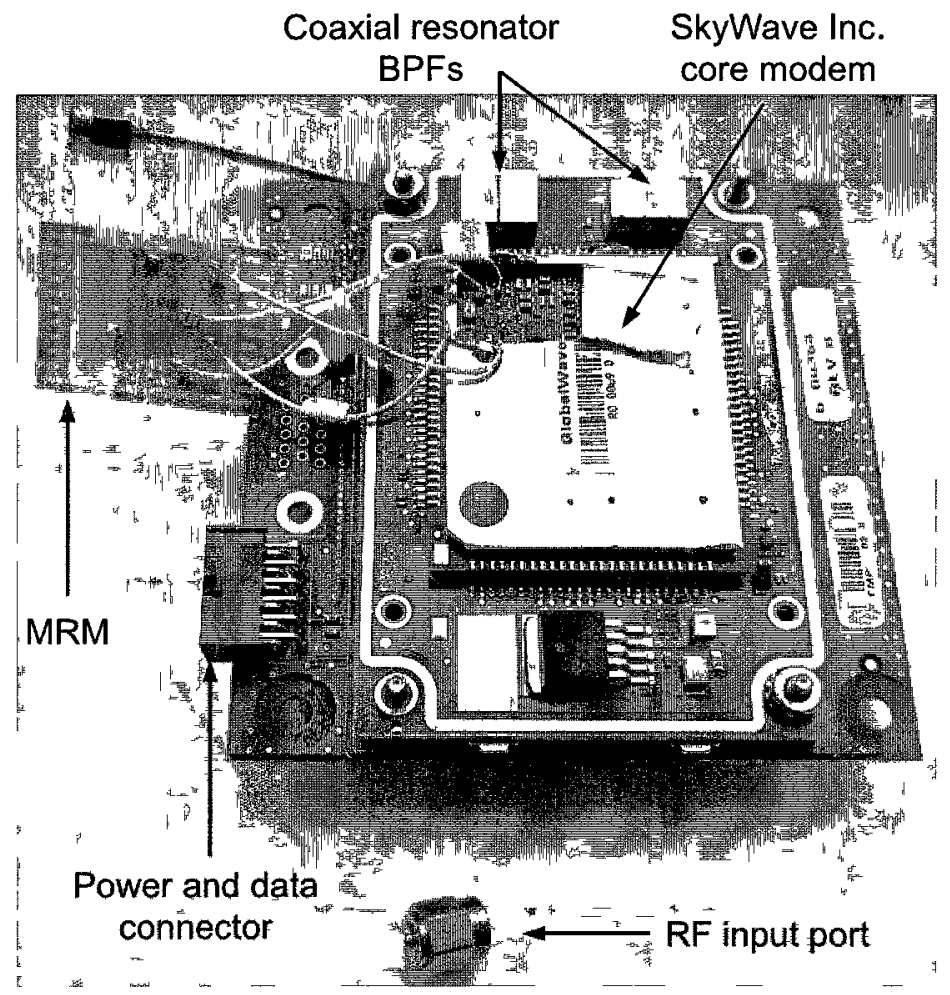

Figure 8.11: MRM and Skywave Inc. measurement setup.

to the use of coaxial resonator type BPFs. These large components are clearly visible 1n Fig. 811

The CM is needed because it provides power and the LO signal to the MRM. First, the CM is tested by itself to characterize its gain, NF and IRR. Then, the front-end of the CM is disabled and the MRM is inserted in its place The same measurements under the same conditions are performed again on this new setup. The goal of this measurement methodology is to provide a good comparison between the MRMI and CM The measurement equipment consists of a IFR 2025 signal generator, HP 8753D network analyzer, HP E4402B spectrum analyzer, and a windows PC An RS232 interface is used between the PC and SkyWave Inc. CM RevB for programming and 
data logging.

In all cases the antennas are bypassed and a signal generator is used to directly supply the CM with an RF signal at $1545 \mathrm{MHz}$. To obtain the desired $\frac{C}{N_{0}}$ value of $37 \mathrm{dBm} / \mathrm{Hz}$, the input signal power is adjusted to $-130 \mathrm{dBm}$. By running a diagnostic program, the performance parameters of the $\mathrm{CM}$ are obtained. Table 8.7 lists typical "in factory" results.

Table 8.6: CM Performance Results (Typical)

\begin{tabular}{|c|c|}
\hline Item & Value $[\mathrm{dB}]$ \\
\hline Gain (front-end) & 30 \\
\hline NF (front-end) & $<2$ \\
\hline IRR & 21 to 27 \\
\hline
\end{tabular}

With the CM front-end disabled, the MRM was able to take its place. Miniature $50 \Omega$ coaxial cables specifically designed for use with WFL connectors were used to connect the MRM with the CM. On the MRM side they were simply "clipped" into place, while the other ends were cut and directly soldered to the correct nodes on the $\mathrm{CM}$. The CM circuitry was exposed by removing the tin shielding in that area. In this way, the MRM front-end effectively replaced the built-in front-end of the CM. Spectrum measurements were first obtained to confirm that the MRM was functioned correctly. For this test, the RF input consisted of a $-100 \mathrm{dBm}$ signal at $1549 \mathrm{MHz}$, while the LO input was set to $1547 \mathrm{MHz}$. The captured IF output spectrums are plotted in Fig. 8.12.

The in-phase output is centered at $2.002 \mathrm{MHz}$ and has an amplitude of $-66.74 \mathrm{dBm}$, while the quadrature output is centered at the same frequency with an amplitude of $-68.16 \mathrm{dBm}$. This result demonstrates that the MRM performs the quadrature 


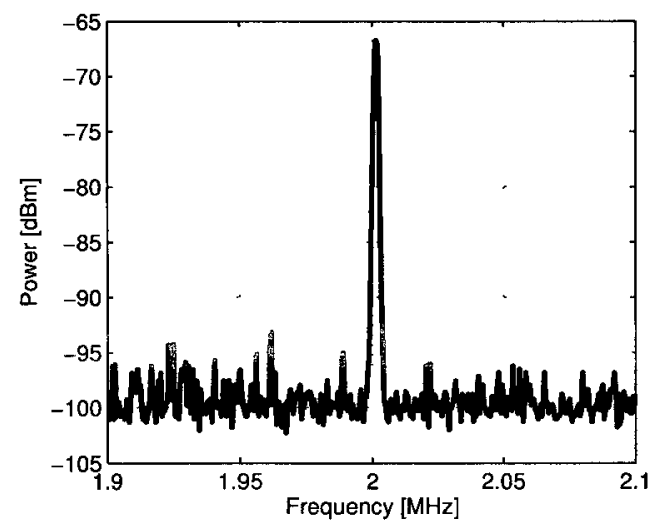

(a)

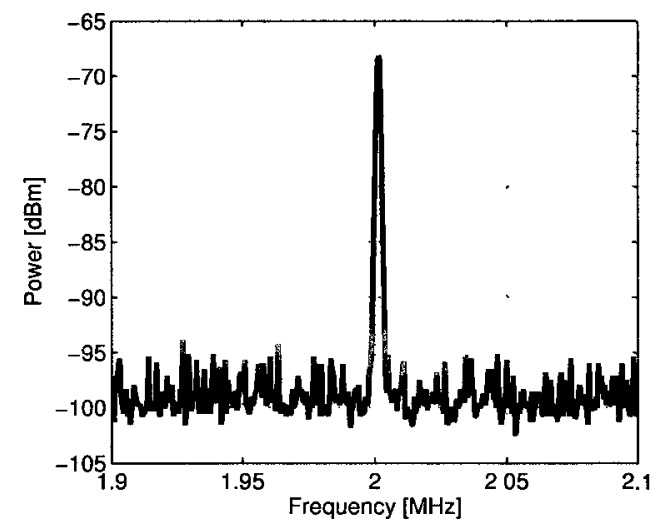

(b)

Figure 8.12: Spectrums of MRM IF outputs (low side LO): (a) I; and (b) Q.

downconversion operation correctly with an amplitude imbalance of approximately $1.4 \mathrm{~dB}$. The difference in amplitudes between the $-100 \mathrm{dBm}$ input signal and the I and $\mathrm{Q}$ outputs can be used to estimate the MRM gain to be at least $34 \mathrm{~dB}$. To obtain the exact gain would require calibrating out the losses of the cables and connectors which may be as much as $5 \mathrm{~dB}$ in total.

From Fig. 8.3, a measured amplitude imbalance of $1.4 \mathrm{~dB}$ would suggest a maximum theoretically achievable IRR of about 17 or $18 \mathrm{~dB}$. This would fall below the target requirements but some of the imbalance may be due to the measurement setup itself. This is likely since the spectrum plots were obtained by connected one IF output to a spectrum analyzer while leaving the other connected to the CM. Differences in impedances at the various interfaces could easily create mismatches that lead to amplitude imbalances. Nevertheless, an IRR test was performed by injecting a tone at the image frequency of $1549 \mathrm{MHz}$ when the RF input is at $1545 \mathrm{MHz}$ and the LO at $1547 \mathrm{MHz}$. As was noted before, a carrier power of $-130 \mathrm{dBm}$ is required to obtain the desired $\frac{C}{N_{0}}$ value of $37 \mathrm{dBm} / \mathrm{Hz}$. Now the IRR can be measured by finding the signal 
power at the image frequency needed to obtain the same $\frac{C}{N_{0}}$ ratio. This was done by slowly increasing the image power from $-130 \mathrm{dBm}$ until the target was achieved. This occurred at a power level of $-107 \mathrm{dBm}$, indicating an IRR of $23 \mathrm{~dB}(130 \mathrm{dBm}$ $107 \mathrm{dBm}$ ). This result is within the target specifications outlined earlier.

Measuring the NF of the MRM is problematic because of the unknown noise levels, especially at the input. Therefore, calculating the SNR degradation would not give an accurate NF. From a first order perspective, the high gain amplifiers effectively buffer-out the noise contribution of subsequent stages. However, noise coupling from the CM to the MRM may be significant since the CM shielding was compromised to allow access to its circuitry. Furthermore, the MRM active components are not shielded. Despite these deficiencies, it is expected that the NF is in the range of $3 \mathrm{~dB}$. Table 8.7 lists the complete MRM results.

Table 8.7: MRM Performance Results (Typical)

\begin{tabular}{|c|c|}
\hline Item & Value $[\mathbf{d B}]$ \\
\hline Gain (front-end) & $>34$ \\
\hline NF (front-end) & 3 (estimate) \\
\hline IRR & 23 \\
\hline
\end{tabular}

In addition to spectrum measurements, a network analyzer was used to verify the performance of the embedded filter and 90-degree hybrid coupler. The following results were obtained through "in measurement" probing, that is while the MRM was powered on and operating normally. A coaxial probe was used to record the s-parameters at the output of the second-order, which were stored in memory. Then, the input s-parameters were recorded and subtracted from those stored in memory to obtain a transfer function. This result is shown in Fig. 8.13 along with a comparison 
with the stand-alone performance first presented in Chapter 4. Without calibration,

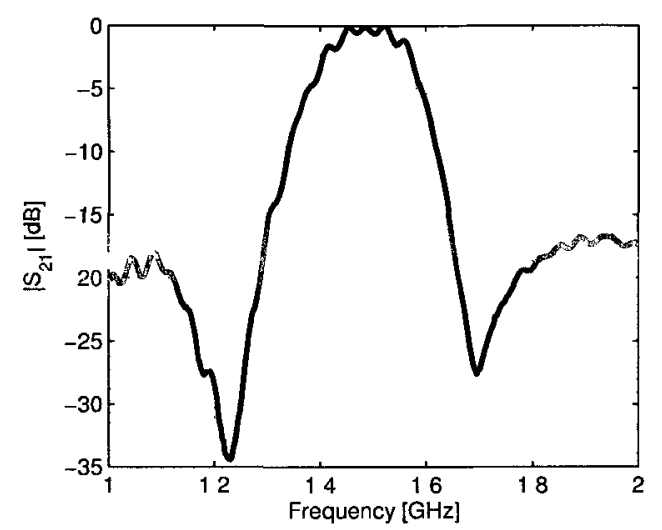

(a)

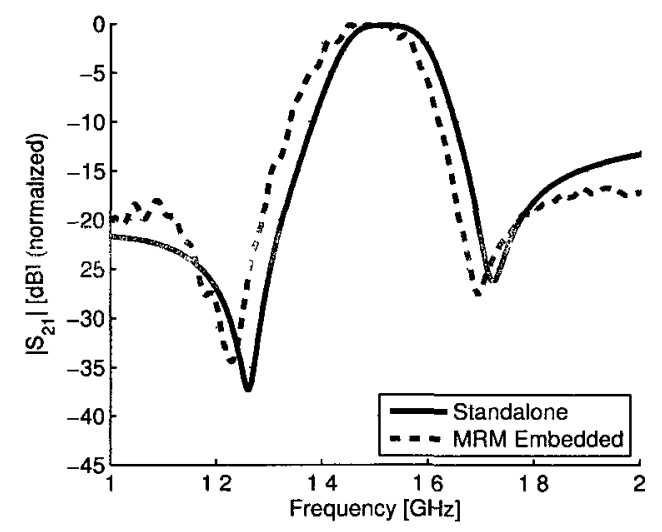

(b)

Figure 8.13: MRM filter measurements: (a) normalized $S_{21}$; and (b) comparison with standalone filter from Chapter 4.

only relative measurements can be obtained so the filter results are normalized. From Fig. 8.13(b), the embedded filters' performance is in very good agreement with the measured $S_{21}$ of its stand-alone counterpart. Only a small positive frequency shift of $40 \mathrm{MHz}$ is observed. This good agreement validates the shielding techniques incorporated in the layout of the MRM and proves that a sub-component can perform adequately within the larger module without any re-design. The same measurement methodology was used to verify the amplitude and phase balance of the 90-degree hybrid coupler. These results are shown in Fig. 8.14. The amplitude imbalance is only $0.42 \mathrm{~dB}$, while the phase difference is 87 degrees. These results compare well with the stand-alone counterpart that displayed an amplitude balance and phase difference of $1.4 \mathrm{~dB}$ and 89 degrees, respectively.

The successful measurement of the MRM represents the first time that a complete LTCC receiver front-end with antenna has been demonstrated for a GPS application. 


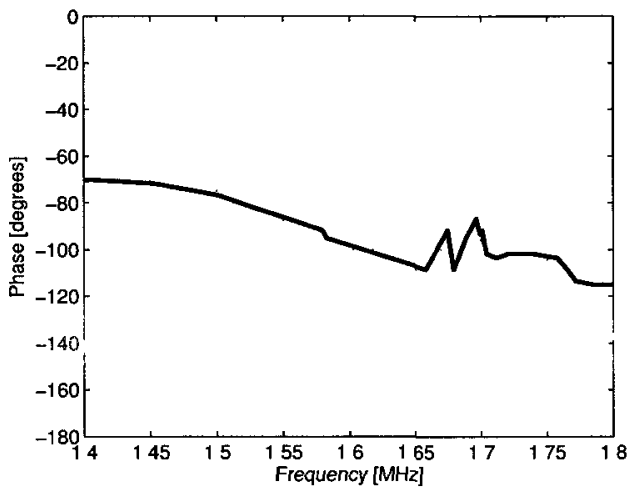

(a)

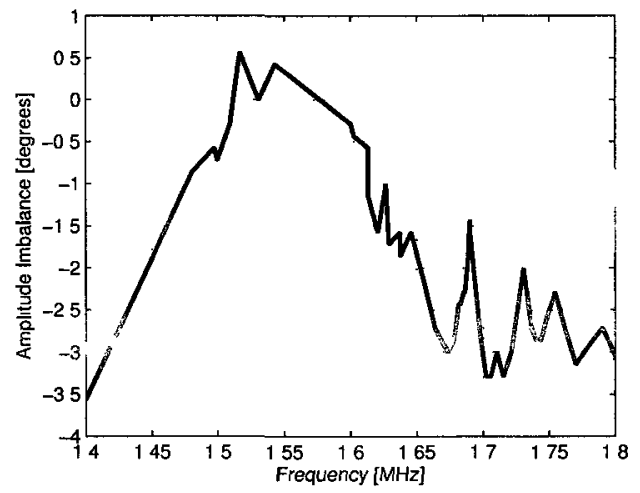

(b)

Figure 8.14: MRM 90-degree coupler measurements: (a) phase difference; and (b) amplitude imbalance.

It has also been proven that each sub-component can perform adequately within the larger module without any re-design if adequate shielding is provided. Even with a very dense module, the concept of "off the shelf" custom passives (i.e. previously developed library elements for a given LTCC environment) can still apply. Finally, these achievements validate SoP as a system-level integration philosophy.

\subsection{Summary and Contribution}

All the previously described receiver components were combined to form a fully functional recelver front-end that completes the fulfilment of the final thesis objective. The MRM is the first demonstration of a fully embedded L-band SoP receiver module with antenna included. A system analysis was performed to determine appropriate specifications and commercial SMT components were chosen to satisfy them. Special layout features including a CPW top layer, via shielding and stripline interconnects were implemented to prove that custom LTCC passives can be embedded within a 
larger module without any re-design. The successful design design, implementation, and experimental characterization of a GPS MRM is seen to validate completely the SoP integration philosophy. The dimensions of the MRM are comparable to a SD memory card or $32 \times 24 \times 2.5 \mathrm{~mm}^{3}$ which makes it compatible with a wide range of existing and future mobile devices. 


\section{Chapter 9}

\section{Thesis Summary and Suggestions for}

\section{Future Work}

This thesis has investigated methods and techniques for the SoP integration of a L-band receiver front-end. Through the combination of custom designed passive components and commercial SMTs, a novel miniature receiver module with antenna has been successfully demonstrated for GPS.

The three main philosophies for system-level integration are presented in Chapter 2. A critical comparison of the three was performed and the philosophy of SoP was seen as the most appropriate for this work. SoP combines the advantages of SoC (low cost) and $\mathrm{SiP}$ (simpler design) to create the potential for a highly integrated, relatively low cost, and high performance design. A GPS application was chosen because its design can benefit greatly from system-level integration techniques and it matches the interests of our industrial partner, SkyWave Inc. Given that SoP has been selected as the design philosophy, an appropriate material system was required. Two of the most commonly used today are LCP and LTCC because of their low loss, good reliability and ability to stack multiple layers. However, it was concluded 
that LTCC is the better choice because it is the more proven of the two technologies. Finally, some high level background information was provided regarding the chosen system and its building blocks such as the bandpass filter, coupler and antenna.

In Chapter 3, the design of the bandpass filter was the first of the building blocks to be undertaken. In the process, a novel methodology was developed not only for filters but lumped element passives in general. Several filter designs were presented culminating in a fully embedded third-order LTCC example that displayed an insertion loss of $2.8 \mathrm{~dB}$ and good out-of-band attenuation. Although reasonable agreement was obtained between measurements and simulations, all sources of error related to LTCC process tolerance effects were analyzed. This was made possible by the inclusion of individual inductors and capacitors that form part of the filter at the time of fabrication. Through their analysis it was concluded that the layer thicknesses and dielectric constant had actual variations of $7 \%$ and $-5 \%$, respectively. These factors contributed greatly to the observed shift in center frequency. In addition, the Qfactors of the spiral inductors were measured to be substantially lower than expected. It was concluded that the higher than expected insertion losses were a direct result of the degraded Q-factors.

The results of Chapter 3, although positive, did leave some room for improvement in the areas of miniaturization and insertion loss. These were addressed in Chapter 4, where more compact topologies and higher $\mathrm{Q}$ inductors were presented. A higher degree of miniaturization was achieved through the application of more advanced filter topologies that include inductor coupling. This technique introduces additional transmission zeros that increase filter selectivity. In this way, a second-order filter was designed that displayed a transfer function almost equivalent to a third-order example but with an insertion loss of only $2.2 \mathrm{~dB}$. A much more selective fourth-order filter 
based on the same principles was also presented. The development of more compact resonator structures was the key to reducing the size of these new filters by $82 \%$, in the case of the second-order design.

The process of designing each building block of the receiver continued in Chapter 5 with the introduction of a 90-degree hybrid coupler. The purpose of this device was to create quadrature signals for I/Q downconversion and demodulation. A lumped element approach was also used to achieve a size reduction of $95 \%$ compared to a conventional coupler. With an insertion loss of only $0.5 \mathrm{~dB}$ and phase balance of 2.5 degrees, this extreme degree of miniaturization did not compromise its performance. Comparisons with commercial products belonging to industry leading companies place the newly developed coupler in a very competitive position.

The building blocks so far presented are single-ended components. However, in the knowledge that the antenna was to be differential, a balun was developed in Chapter 6 to perform the necessary conversion. Its design is based on the same principles as the 90-degree hybrid coupler whereby transmission lines were replaced with lumped equivalents. This again resulted in a highly miniaturized and good performing device whose characteristics make it very competitive with leading commercial counterparts. The balun's low $0.67 \mathrm{~dB}$ insertion loss and very stable phase balance of 0.25 degrees were key elements in ensuring that the antenna performed as expected.

In Chapter 7, a dipole antenna was co-designed with the previously described balun to form a combined antenna-balun component. Based on a comparison of several elements, a dipole was chosen partly because it does not require a ground plane to radiate efficiently. Miniaturization techniques such as folding and meandering were used so that plenty of space on the receiver module could be allocated to the other components. Full pattern measurements were performed at the Canadian Space 
Agency's facilities at CRC and indicate gain levels comparable to that of commercially available SMT antennas.

The building blocks of the previous chapters were combined in Chapter 8 that presented a stand-alone miniature receiver module (MRM). The dimensions of the MRM are comparable to a SD memory card or $32 \times 24 \times 2.5 \mathrm{~mm}^{3}$ and within this module all the previously described components were embedded. Additional surface mount active components were added to create a functional receiver front-end. The MRM layout was carefully planned to minimize the lengths of all interconnects while special attention was paid to electromagnetically isolate each component. Shielding techniques such as employing CPW lines on exposed layers, stripline interconnects in embedded layers, and via fences worked to minimize unwanted coupling. SkyWave Inc.'s participation was key to obtaining measurements of the MRM since an interface board was provided that avoided the need for specialized test fixtures. It was concluded that the MRM's performance is comparable to a commercial equivalent whose size is many times larger.

The contents of chapters 2 to 7 dealt with the design and characterization of individual components for the receiver. These were then combined to form the MRM described in Chapter 8. The first thesis objective, that of comparing three systemlevel integration techniques, was met with the selection of the SoP philosophy as the one which would allow for the best realization of the MRM. Instead of classical distributed circuits, lumped element equivalents were used to reduce their size significantly. In fulfillment of the second thesis objective, a methodology was developed for the efficient design of lumped element passive structures. The methodology relies on the ability to replace, in simulations, some embedded components with ideal impedance boundaries whose values are fixed and immune to mutual coupling. Based 
on this methodology, a series of passive components was designed (filters, coupler, balun) which constituted the fulfilment of the third thesis objective. Finally, the new MRM was implemented with an antenna in SoP form. The antenna was characterized separately from the rest of the receiver and provided a peak gain of $-0.8 \mathrm{~dB}$ at $1.55 \mathrm{GHz}$. The receiver itself was found to correctly down-convert the RF signal while providing sufficient system gain and acceptable noise performance. The successful operation of the antenna and receiver was seen to validate the fourth and final thesis objective, namely the co-design and aggressive miniaturization of a SoP receiver front-end with antenna.

This is the first time that a complete LTCC receiver front-end with antenna has been demonstrated for a GPS application. It has also been proven that each subcomponent can perform adequately within the larger module without any re-design. This implies that the concept of "off the shelf" custom passives has been shown. These achievements validate SoP as a system-level integration philosophy.

Although the objectives of this work have been achieved, five suggestions are given next for obtaining improved results.

The planar spiral inductors developed in Chapter 3 displayed significantly degraded Q-factors. This situation repeated itself in the inductor designs of chapter 4, 5 , and 6 , albeit to a lesser degree. The causes of this issue should be investigated and corrected in future designs. A direct benefit of this would be lower insertion losses of all filters, in particular.

Although good agreement between simulations and measurements was achieved throughout, improvements should be made if de-embedding of the G-S-G pads is performed. Any of the well known techniques could be used to reveal the true performance of the device under test. 
Due to process and component tolerances, it was challenging to make the antenna resonant at the desired frequency. Another complicating factor was its narrow bandwidth. In order to avoid this problem, a wider band antenna should be used. This could be accomplished by increasing the volume of the dipole or by employing a higher order matching network.

Due to unknown noise levels, the NF of the MRM could not be measured accurately. Improving the shielding of the MRM by placing the active components in cavities and sealing them with metal lids could allow for reliable NF measurements.

To further reduce the size and performance of the MRM, several enhancements should be made. A mixed dielectric material system that incorporates high dielectric layers would allow for smaller antenna elements and larger capacitors. Meanwhile, a custom RFIC that includes image rejection along with adaptive amplitude and phase correction would perform better than the current SMT mixers.

The above suggestions are intended for the refinement of the current work to make it more suitable for commercialization. However, the design principles and passive components, in particular the 90-degree hybrid coupler, can be used for a different application such as a six-port receiver. A six port receiver is a type of direct conversion receiver that uses three 90-degree hybrid couplers to create four relative phase differences between the input RF and LO signals. These signals are then superimposed and downconverted to baseband where the I and Q information can be recovered. The six-port has several advantages that make it an attractive alternative to traditional heterodyne microwave receivers but its large size (mainly due to the couplers) is a major drawback. Therefore, the miniaturization techniques and the hybrid coupler presented in Chapter 5 could be ideal solutions to this problem. 


\section{List of References}

[1] M. Lazarus, "The great spectrum famine," IEEE Spectrum, pp. 26-31, October 2010.

[2] I. Bahl, Lumped Elements for RF and Microwave Circuits. Artech House, 2003.

[3] J. M.Lopez-Villegas, J. Samitier, C. C. PereLosantos, and J. Bausells, "Improvement of the quality factor of RF integrated inductors by layout optimization," IEEE Transactions on Microwave Theory and Techniques, vol. 48, pp. 76-83, January 2000.

[4] A. Malm, "GPS and mobile handsets," tech. rep., Berg Insight, 2008.

[5] G. Brzezina, L. Roy, and L. MacEachern, "Design enhancement of miniature lumped-element LTCC bandpass filters," IEEE Transactions on Microwave Theory and Techniques, vol. 57, pp. 815-823, June 2009.

[6] G. Brzezina, L. Roy, and L. MacEachern, "A miniature LTCC filter using novel resonators for GPS applications," in European Microwave Conference, pp. 536539, October 2007.

[7] G. Brzezina and L. Roy, "A miniature lumped element LTCC bandpass filter with high stopband attenuation for GPS applications," in IEEE MTT-S International Microwave Symposium, pp. 1215-1218, June 2008.

[8] G. Brzezina and L. Roy, "A miniature lumped element LTCC quadrature hybrid coupler for GPS applications," in IEEE Antennas and Propagation Symposium, pp. 1-4, July 2008. 
[9] G. M. Brzezina and L. Roy, "A miniaturized GPS antenna in LTCC with linear polarization suitable for SoP integration," Antenna Technology and Applied Electromagnetics \& the American Electromagnetics Conference (ANTEMAMEREM), pp. 1-4, July 2010.

[10] J. Wight, J. L. L. Carley, and T. Riley, "On-die synthesized inductors: Boon or bane?," IEEE Microwave Magazine, vol. 11, pp. 95-104, May 2010.

[11] R. Tummala, "Packaging: past, present and future," in Electronic Packaging Technology Conference, pp. 3-7, August 2005.

[12] K. Lyne, "Custom integrated circuits conference," in Electronic Packaging Technology, pp. 765-770, September 2005.

[13] R. Tummala, M. Swaminathan, M. Tentzeris, J. Laskar, G.-K. Chang, S. Sitaraman, D. Keezer, D. Guidotti, Z. Huang, K. Lim, L. Wan, S. Bhattacharya, V. Sundaram, F. Liu, and P. Raj, "The SOP for miniaturized, mixed-signal computing, communication, and consumer systems of the next decade," IEEE Transactions on Advanced Packaging, vol. 27, pp. 250-267, May 2004.

[14] S. Lim, "Physical design for 3D system on package," IEEE Design and Test of Computers, vol. 22, pp. 532-539, November 2005.

[15] V. Sundaram, R. Tummala, G. White, K. Lim, L. Wan, D. Guidotti, F. Liu, S. Bhattacharya, R. Pulugurtha, I. Abothu, R. Doraiswami, R. Pucha, J. Laskar, M. Tentzeris, G. Chang, and M. Swaminathan, "System-on-a-package (SOP) substrate and module with digital, RF and optical integration," in Electronic Components and Technology Conference, vol. 1, pp. 17-23, June 2004.

[16] B. Kopp and A. Francomacaro, "Miniaturized stripline circuitry utilizing low temperature cofired ceramic (LTCC) technology," Microwave Symposium Digest, vol. 3, pp. 1513-1516, 1992.

[17] D. C.Thompson, O. Tantot, H. Jallageas, G. E.Ponchak, M. M.Tentzeris, and J. Papapolymerou, "Characterization of liquid crystal polymer (LCP) material and transmission lines on LCP substrates from 30 to $110 \mathrm{ghz}$," IEEE Transactions on Microwave Theory and Techniques, vol. 52, pp. 1343-1352, April 2004. 
[18] A. Shamim, J. Bray, and N. H. R. E. D. Baillargeat, "Microwave and magnetostatic characterization of ferrite LTCC for tunable and reconfigurable SiP applications," Microwave Symposium, pp. 691-694, 2007.

[19] W. Myrick, M. Zoltowski, and J. Goldstein, "GPS jammer suppression with lowsample support using reduced-rankpower minimization," in Statistical Signal and Array Processing Workshop, vol. 1, pp. 514-518, August 2000.

[20] Y.-H. Jeng, S.-F. Chang, and H.-K. Lin, "A high stopband-rejection LTCC filter with multiple transmission zeros," IEEE Transactions on Microwave Theory and Techniques, vol. 54, pp. 633-638, June 2005.

[21] C. Tang and S. You, "Design methodologies of LTCC bandpass filters, diplexers, and triplexer with transmission zeros," IEEE Transactions on Microwave Theory and Techniques, vol. 54, pp. 717-723, February 2006.

[22] A. Sutono, J. Laskar, and W. Smith, "Design of miniature multilayer on-package integrated image-reject filters," IEEE Transactions on Microwave Theory and Techniques, vol. 51, pp. 156 - 162, January 2003.

[23] D. M. Pozar, Microwave Engineering. Wiley, 3rd ed., 2005. Ch. 6.

[24] H. Handtmann, S. Marksteiner, J. Kaitila, and R. Aigner, "Bulk acoustic wave filters for GPS with extreme stopband attenuation," IEEE MTT-S Digest, vol. 1, pp. 371-374, June 2004.

[25] A. Escobar, W. McGinnis, M. Pulling, and H. Whitehouse, "SAW filter for global positioning satelite," in IEEE Position Location and Navigation Symposium, vol. 1, pp. 4-11, April 2002.

[26] R. Tan and R. Levy, "A limiting filter for application is a GPS receiver," IEEE MTT-S International Microwave Symposium Digest, vol. 1, pp. 881-883, June 1993.

[27] W.-S. Tung, Y.-C. Chiang, and J.-C. Cheng, "A new compact LTCC bandpass filter using negative coupling," IEEE Microwave and Wireless Components Letters, vol. 15, pp. 641-643, October 2005. 
[28] K. Rambabu and J. Bornemann, "Simplified analysis technique for the initial design of LTCC filters with all-capacitive coupling," IEEE Transactions on Microwave Theory and Techniques, vol. 53, pp. 1787-1791, May 2005.

[29] C. Lee and H. Huang, "Design of a new bandpass filter using anti-parallel coupled asymmetric SIRs," IEICE Trans. Electron., vol. E89-C, pp. 571-575, April 2006.

[30] B. R. Heimer, L. Fan, and K. Chang, "Uniplanar hybrid couplers using asymmetrical coplanar striplines," IEEE Transactions on Microwave Theory and Techniques, vol. 45, pp. 2234-2240, December 1997.

[31] L. Chiu, Q. Xue, and C. H. Chan, "The multilayer folded couplers and their application," Microwave and Optical Technology Letters, vol. 48, pp. 2285-2288, November 2006.

[32] C.-W. Tang and M.-G. Chen, "Reduced-size branch-line and rat-race hybrids for uniplanar MMIC's," IEEE Transactions on Microwave Theory and Techniques, vol. 38, pp. 270-275, March 1990.

[33] P.-L. Chi, R. Waterhouse, and T. Itoh, "Antenna miniaturization using slow wave enhancement factor from loaded transmission line models," IEEE Transactions on Antennas and Propagation, vol. 59, pp. 48-57, January 2011.

[34] Y. Zhang and H. D. Yang, "Ultra slow-wave periodic transmission line using 3D substrate metallization," in IEEE MTT-S Microwave Symposium Digest, pp. 891-894, June 2008.

[35] P. Popplewell, V. Karam, A. Shamim, J. Rogers, and C. Plett, "An injectionlocked 5.2 GHz SoC transceiver with on-chip antenna for self-powered RFID and medical sensor applications," in IEEE Symposium on VLSI Circuits, pp. 88-89, June 2007.

[36] A. Shamim, L. Roy, N. Fong, and N. Tarr, "24 GHz on-chip antennas and balun on bulk si for air transmission," IEEE Transactions on Antennas and Propagation, vol. 56, pp. 303-311, February 2008.

[37] C. Cao, Y. Ding, X. Yang, J.-J. Lin, A. Verma, J. Lin, F. Martin, and K. O, "A 24-GHz transmitter with an on-chip antenna in 130-nm CMOS," in VLSI Circuits Symposium, pp. 148-149, 2006. 
[38] M. R. N. Ahmadi, S. Safavi-Naeini, and L. Zhu, "An efficient CMOS on-chip antenna structure for system in package transceiver applications," IEEE Radio and Wireless Symposium, pp. 487-490, January 2007.

[39] A. Holub and M. Polivka, "A novel microstrip patch antenna miniaturization technique: A meanderly folded shorted-patch antenna," in Conference on $\mathrm{Mi}$ crowave Techniques, pp. 1-4, April 2008.

[40] A. Serra, P. Nepa, G. Manara, and R. Massini, "A low-profile linearly polarized 3D PIFA for handheld GPS terminals," IEEE Transactions on Antennas and Propagation, vol. 58, pp. 1060-1066, April 2010.

[41] V. Pathak, S. Thornwall, M. Krier, S. Rownson, G. Poilasne, L. Desclos, and H. Miller, "Mobile handset system performance comparison of a linearly polarized GPS internal antenna with a circularly polarized antenna," IEEE Transactions on Antennas and Propagation, vol. 3, pp. 666-669, 2003.

[42] Q. Luo, H. Salgado, and J. Pereira, "EBG PIFA design for WLAN applications," in Antennas and Propagation Society Symposium, pp. 1-4, July 2008.

[43] P.-L. Teng, S.-T. Fang, and K.-L. Wong, "PIFA with a bent, meandered radiating arm for GSM/DCS dual-band operation," in Antennas and Propagation Society Symposium, pp. 1-4, June 2003.

[44] I. Zuazola, J. Batchelor, and R. Langley, "Miniaturised multiband PIFA antenna using a frequency selective surface (FSS) ground plane," in Antennas and Propagation Conference, pp. 281-284, April 2007.

[45] Y.-S. Wang, M.-C. Lee, and S.-J. Chung, "Two PIFA-related miniaturized dualband antennas," IEEE Transactions on Antennas and Propagation, vol. 55, pp. 805-811, March 2007.

[46] D.-Y. Kim, J. Lee, C. S. Cho, and T. Lee, "Design of a compact tri-band PIFA based on independent control of the resonant frequencies," IEEF Transactions on Antennas and Propagation, vol. 56, pp. 1428-1436, May 2008.

[47] C.-W. Ling, C.-Y. Lee, C.-L. Tang, and S.-J. Chung, "Analysis and application of an on-package planar inverted-f antenna," IEEE Transactions on Antennas and Propagation, vol. 55, pp. 1774 - 1780, June 2007. 
[48] G. Lui and R. Murch, "Compact dual-frequency PIFA designs using LC resonators," IEEE Transactions on Antennas and Propagation, vol. 49, pp. 1016 - 1019, July 2001.

[49] B. Sanz-Izquierdo, J. Batchelor, R. Langley, and M. Sobhy, "Single and double layer planar multiband PIFAs," IEEE Transactions on Antennas and Propagation, vol. 54, pp. 1416 - 1422, May 2006.

[50] S. R. Best, "A discussion on small antennas operating with small finite ground planes," IEEE International Workshop on Antenna Technology Small Antennas and Novel Metamaterials, pp. 152-155, March 2006.

[51] M.-C. Huynh and W. Stutzman, "Ground plane effects on planar inverted-f antenna (PIFA) performance," IEE Microwaves, Antennas and Propagation, vol. 150, pp. 209-213, August 2003.

[52] Y. Zhou, C.-C. Chen, and J. L. Volakis, "Single-fed circularly polarized antenna element with reduced coupling for GPS arrays," IEEE Transactions on Antennas and Propagation, vol. 56, pp. 1469-1472, May 2008.

[53] G. Z. Rafi, M. Mohajer, A. Malarky, P. Mousavi, and S. Safavi-Naeini, "Lowprofile integrated microstrip antenna for GPS-DSRC application," IEEE Antennas and Wireless Propagation Letters, vol. 8, pp. 44-48, January 2009.

[54] X. L. Bao, G. Ruvio, M. J. Ammann, and M. John, "A novel GPS patch antenna on a fractal hi-impedance surface substrate," IEEE Antennas and Wireless Propagation Letters, vol. 5, pp. 323-326, July 2006.

[55] chen, "Compact dual-band GPS microstrip antenna using multilayer LTCC substrate," IEEE Antennas and Wireless Propagation Letters, vol. 9, pp. 421423, May 2010.

[56] Y. Zhou, C.-C. Chen, and J. L. Volakis, "Dual band proximity-fed stacked patch antenna for tri-band GPS applications," IEEE Transactions on Antennas and Propagation, vol. 55, pp. 220-223, January 2007.

[57] N. Padros, J. I. Ortigosa, J. Baker, M. F. Iskander, , and B. Thornberg, "Comparative study of high-performance GPS receiving antenna designs," IEEE Transactions on Antennas and Propagation, vol. 45, pp. 698-706, April 1997. 
[58] C. A. Balanis, Antenna Theory Analysis and Design. John Wiley \& Sons, Inc., 3rd ed., 2005.

[59] D. Roscoe, L. Shafai, A. Ittipiboon, M. Cuhaci, and R. Douville, "Tunable dipole antennas," in Antennas and Propagation Society Internation Symposium, vol. 2, pp. 672-675, June 1993.

[60] S.-G. Kim and K. Chang, "Frequency tunable CPW-fed slot dipole using piezoelectric transducer," Electronics Letters, vol. 39, pp. 991--992, June 2003.

[61] A. Kolsrud, M.-Y. Li, and K. Chang, "Dual-frequency electronically tunable CPW-fed CPS dipole antenna," Electronics Letters, vol. 34, pp. 609-611, April 1998.

[62] F. Dantoni, A. Holden, S. Fu, D. Sahu, S. Venkatraman, and S. Embabi, "A highly integrated GPS receiver for cellular handset," in Radio Frequency Integrated Circuits Symposium, pp. 93-96, May 2001.

[63] S. Gunnarsson, D. Kuylenstierna, and H. Zirath, "Analysis and design of millimeter-wave FET-based image reject mixers," IEEE Transactions on Microwave Theory and Techniques, vol. 55, pp. 2065 - 2074, October 2007.

[64] D. Guermandi, P. Tortori, E. Franchi, and A. Gnudi, "A 0.83-2.5-GHz continuously tunable quadrature VCO," IEEE Journal of Solid-State Circuits, vol. 40, pp. 2620 - 2627, December 2005.

[65] J. Macedo and M. Copeland, "A 1.9-GHz silicon receiver with monolithic image filtering," IEEE Journal of Solid-State Circuits, vol. 33, pp. 378-386, March 1998.

[66] J. Rogers, J. Macedo, and C. Plett, "A completely integrated $1.9 \mathrm{GHz}$ receiver front-end with monolithic image reject filter and VCO," in Radio Frequency Integrated Circuits Symposium, pp. 143-146, June 2000.

[67] B. Razavi, RF Microelectronics. Prentice Hall PTR, 1998. Ch. 5.

[68] T.-S. Horng, J.-M. Wu, L.-Q. Yang, and Shyh-Tirng, "A novel modified-t equivalent circuit for modeling LTCC embedded inductors with large bandwidth," IEEE Transactions on Microwave Theory and Techniques, vol. 51, pp. 23272333, December 2003. 
[69] M. Ma and R. Khazaka, "Model order reduction with parametric port formulation," IEEE Transactions on Advanced Packaging, vol. 30, pp. 763-775, November 2007.

[70] W. Tseng, C. Chen, E. Gad, M. Nakhla, and R. Achar, "Passive order reduction for RLC circuits with delay elements," IEEE Transactions on Advanced Packaging, vol. 30, pp. 830-840, November 2007.

[71] N. Nakhla, A. Dounavis, M. Nakhla, and R. Achar, "Delay-extraction-based sensitivity analysis of multiconductor transmission lines with nonlinear terminations," IEEE Transactions on Microwave Theory and Techniques, vol. 53, pp. 3520-3530, November 2005.

[72] G. Shinh, R. Achar, N. Nakhla, M. Nakhla, and I. Erdin, "Simplified macromodel of MTLs with incident fields (SiMMIF)," IEEE Transactions on Electromagnetic Compatibility, vol. 50, pp. 375-389, May 2008.

[73] G. Shinh, N. Nakhla, R. Achar, M. Nakhla, A. Dounavis, and I. Erdin, "Fast transient analysis of incident field coupling to multiconductor transmission lines," IEEE Transactions on Electromagnetic Compatibility, vol. 48, pp. 57-73, February 2006.

[74] A. Simine, D. Kholodnyak, P. Turalchuk, V. Piatnitsa, H. Jantunen, and I. Vendik, "Enhancement of inductance Q-factor for LTCC filter design," in European Microwave Conference, vol. 2, October 2005.

[75] G. Brzezina, L. Roy, and L. MacEachern, "Planar antennas in LTCC technology with transceiver integration capability for ultra-wideband applications," IEEE Transactions on Microwave Theory and Techniques, vol. 54, pp. 2830-2839, June 2006.

[76] J. Wight, J. Long, L. R. Carley, and T. Riley, "On-die synthesized inductors: Boon or bane?," IEEE Microwave Magazine, vol. 11, pp. 95-104, May 2010.

[77] C. Tang and C. Chang, "LTCC-MLC chip-type balun realised by LC resonance method," Electronic Letters, vol. 38, pp. 519-520, MayJune 2002.

[78] K. Kautio, "Design guidelines low temperature co-fired ceramic modules," tech. rep., VTT Electronics, May 2004. version 1.2. 
[79] A. B. Carlson and D. G. Gisser, Electrical Engineering Concepts and Applications. Addison-Wesley, 1981.

[80] L. K. Yeung and K.-L. Wu, "Design of a 2-pole LTCC filter for wireless communications," IEEE Transactions on Wireless Communications, vol. 3, pp. 379381, March 2004.

[81] C.-W. Tang, "Harmonic-suppression LTCC filter with the step-impedance quarter-wavelength open stub," IEEE Transactions on Microwave Theory and Techniques, vol. 52, pp. 617-624, February 2004.

[82] L. K. Yeung and K.-L. Wu, "A compact second-order LTCC bandpass filter with two finite transmission zeros," IEEE Transactions on Microwave Theory and Techniques, vol. 51, pp. 337-341, February 2003.

[83] J. F. Valenzuela-Valdes, M. A. Garcia-Fernandez, A. M. Martinez-Gonzalez, and D. Sanchez-Hernandez, "The role of polarization diversity for MIMO systems under rayleigh-fading environments," IEEE Antennas and Wireless Propagation Letters, vol. 5, pp. 534-536, November 2006.

[84] B. Yuan, W. Yu, H. Sun, and X. Lv, "A bandpass filter using LTCC system-onpackage (SOP) technology," Microwave Technology and Computational Electromagnetics, pp. 257-260, November 2009.

[85] Y.-S. Dai, H.-S. Zhang, G.-P. Qi, D.-L. Lu, Z.-L. Tao, G.-X. Qian, and H. Wang1, "LTCC bandpass filter for bluetooth application with dual transmission zeros," Microwave and Millimeter Wave Technology, pp. 284-286, April 2008 .

[86] M. Hft and T. Shimamura, "Design of symmetric trisection filters for compact low-temperature co-fired ceramic realization," IEEE Transactions on $\mathrm{Mi}$ crowave Theory and Techniques, vol. 58, pp. 165-175, January 2010.

[87] R. Kravchenko, D. Orlenko, and M. Stadler, "A new miniaturized LTCC transversal bandpass filter for RF and microwave applications," Communucatıon Systems, Networks and Digital Signal Processing, pp. 398-401, July 2008.

[88] H.-C. Hsu, T.-B. Hou, W.-H. Huang, and C.-W. Tang, "The multilayered bandpass filter with multiple transmission zeros," Asia Pacific Microwave Conference, pp. 2549 - 2552, December 2009. 
[89] C.-W. Tang, C.-W. Shen, and P.-J. Hsieh, "Design of low-temperature co-fired ceramic bandpass filters with modified coupled inductors," IEEE Transactions on Microwave Theory and Techniques, vol. 57, pp. 172-179, January 2009.

[90] B. Yuan, W. Yu, H. Sun, and X. Lv, "A LTCC band pass filter for wireless applications," Microwave, Antenna, Propagation and EMC Technologies for Wireless Communications, pp. 470-472, October 2009.

[91] M. Danesh, J. Long, R. Hadaway, and D. Harame, "A Q-factor enhancement technique for MMIC inductors," IEEE MTT-S Digest, vol. 1, pp. 183-186, 1998.

[92] N. Kidera, S. Pinel, S. Watanabe, K. Watanabe, and J. Laskar, "Wideband, low loss and compact 3D LTCC balun with asymmetric structure for millimeter wave applications," IEEE Transactions on Microwave Theory and Techniques, vol. 3, pp. 547-550, June 2005.

[93] Y. Guo, Z. Zhang, and L. Ong, "Design of miniaturized LTCC baluns," IEEE Transactions on Microwave Theory and Techniques, vol. 3, pp. 1567-157, June 2006.

[94] T.-G. Kim and B. Lee, "Metamaterial-based wideband rat-race hybrid coupler using slow wave lines," IET Microwaves, Antennas and Propagation, vol. 4, no. 6, pp. $717-721,2010$.

[95] S. Parisi, " $180^{0}$ lumped element hybrid," IEEE Transactions on Microwave Theory and Techniques, vol. 3, pp. 1243-1246, June 1989.

[96] R. Perrone, P. Kapitanova, D. Kholodnyak, I. Vendik, S. Humbla, M. Hein, and J. Mller, "Miniaturisation of a LTCC high-frequency rat-race-ring by using 3-dimensional integrated passives and embedded high-K capacitors," European Microelectronics and Packaging Conference, pp. 1-6, June 2009.

[97] K. Markov, S. Royak, G. Sevskiy, and P. Heide, "A simple LTCC balun for WLAN applications using left-handed (LH) transmission lines (TL)," European Microwave Conference, pp. 141-144, October 2005.

[98] S. Sakhnenko, D. Orlenko, K. Markov, A. Yatsenko, B. Vorotnikov, G. Sevskiy, P. Heide, and M. Vossiek, "Low profile LTCC balanced filter based on a lumped elements balun for WiMAX applications," IEEE Transactions on Microwave Theory and Techniques, pp. 1111-1114, June 2008. 
[99] Y. J.Yoon, Y. Lu, R. C.Frye, and PeterR.Smith, "Modeling of monolithic RF spiral transmission-line balun," IEEE Transactions on Microwave Theory and Techniques, vol. 49, pp. 393-395, February 2001.

[100] D. Kuylenstierna and P. Linnr, "Design of broad-band lumped-element baluns with inherent impedance transformation," IEEE Transactions on Microwave Theory and Techniques, vol. 52, pp. 2739-2745, December 2004.

[101] J. Gianvittorio and Y. Rahmat-Samii, "Fractal antennas: a novel antenna miniaturization technique, and applications," IEEE Antennas and Propagation Magazine, vol. 44, pp. 20-36, February 2002.

[102] F. Namin, T. Spence, D. Werner, and E. Semouchkina, "Broadband, miniaturized stacked-patch antennas for l-band operation based on magneto-dielectric substrates," IEEE Transactions on Antennas and Propagation, vol. 58, pp. 2817-2822, September 2010.

[103] K. Buell, H. Mosallaei, and K. Sarabandi, "A substrate for small patch antennas providing tunable miniaturization factors," IEEE Transactions on Microwave Theory and Techniques, vol. 54, pp. 135-146, September 2006.

[104] J. Volakis, C.-C. Chen, and K. Fujimoto, Small Antennas:Miniaturization Techniques \& Applications. McGraw-Hill Professional, 1 ed., June 2010.

[105] J. Volakis, Antenna Engineering Handbook. McGraw-Hill Professional, 4 ed., June 2007.

[106] B. Kramer, C.-C. Chen, M. Lee, and J. Volakis, "Fundamental limits and design guidelines for miniaturizing ultra-wideband antennas," IEEE Antennas and Propagation Magazine, vol. 51, pp. 57-69, August 2009.

[107] A. Skrivervik, J.-F. Zurcher, O. Staub, and J. Mosig, "Pcs antenna design: the challenge of miniaturization," IEEE Antennas and Propagation Magazine, vol. 43, pp. 12-27, August 2001.

[108] T. G. Spence and D. H. Werner, "A novel miniature broadband/multiband antenna based on an end-loaded planar open-sleeve dipole," IEEE Transactions on Antennas and Propagation, vol. 54, pp. 3614-3620, December 2006. 
[109] B. Ghosh, S. Haque, D. Mitra, and S. Ghosh, "A loop loading technique for the miniaturization of non-planar and planar antennas," IEEE Transactions on Antennas and Propagation, vol. 58, pp. 2116-2121, June 2010.

[110] J. S. Petko and D. H. Werner, "Miniature reconfigurable three-dimensional fractal tree antenna," IEEE Transactions on Antennas and Propagation, vol. 52, pp. 1945-1956, August 2004.

[111] L. Chu, "Physical limitations of omnidirectional antennas," J. Appl. Phys., vol. 34, pp. 1479-1484, December 1947.

[112] S. R. Best, "The significance of ground-plane size and antenna location in establishing the performance of ground-plane-dependent antennas," IEEE Antennas and Propagation Magazine, vol. 51, pp. 29-43, December 2009.

[113] Y. Zhang and H. Y. D. Yang, "Bandwidth-enhanced electrically small printed folded dipoles," IEEE Antennas and Wireless Propagation Letters, vol. 9, pp. 236-239, March, 2010.

[114] T. Endo, Y. Sunahara, S. Satoh, and T. Katagi, "Resonant frequency and radiation efficiency of meander line antennas," Electronics and Communications in Japan, vol. 83, no. 1, pp. 52-58, 2000.

[115] T. J. Wamagirism and T. J. Minardo, "Performance of a meandered line as an electrically small transmitting antenna," IEEE Transactions on Antennas and Propagation, vol. 46, pp. 1797-1801, December 1998.

[116] V. Rabinovich, B. Al-Khateeb, B. Oakley, and N. Alexandrov, "Small printed meander symmetrical and asymmetrical antenna performances, including the RF cable effect, in the $315 \mathrm{MHz}$ frequency band," Microwave and Optical Technology Letters, vol. 48, pp. 1828-1833, September 2006.

[117] K. Fujimoto, Small Antennas. John Wiley and Sons, New York, 1987.

[118] Y.-T. Im, J.-H. Kim, and W.-S. Park, "Matching techniques for miniaturized UHF RFID loop antennas," IEEE Antennas and Wireless Propagation Letters, vol. 8, pp. 266-270, May 2009. 
[119] J. Ko, J. Kim, S. Cho, and K. Lee, "A $19 \mathrm{~mW} 2.6 \mathrm{~mm}^{2}$ L1/L2 dual-band CMOS GPS receiver," IEEE Journal of Solid-State Circuits, vol. 40, pp. 1414-1425, July 2005.

[120] A. C. Heiberg, T. W. Brown, T. S. Fiez, and K. Mayaram, "A $250 \mathrm{mV}, 352 \mu \mathrm{W}$ GPS receiver RF front-end in $130 \mathrm{~nm}$ CMOS," IEEE Journal of Solid-State Circuits, vol. 46, pp. 938-949, April 2011. 